

\section{MINIATURIZED ELECTROCHEMICAL CELLS FOR APPLICATIONS IN DRUG SCREENING AND PROTEIN CLEAVAGE}

IR. M. ODIJK

4 MARCH 2011

\section{Chair:}

BIOS - The Lab-on-a-Chip Group

Faculty:

EWI, University of Twente 
The research described in this thesis was carried out at the BIOS Lab-on-aChip group of the MESA+ institute for Nanotechnology of the University of Twente, Enschede, The Netherlands. It was carried out in close cooperation with the Institute of Inorganic and Analytical Chemistry of the University of Münster, Münster, Germany and the Analytical Biochemistry group and the Mass Spectrometry Core Facility of the Centre of Pharmacy of the University of Groningen, Groningen, The Netherlands. The research was financially supported by the Dutch Technology Foundation (STW) as project 07047.

Members of the committee:

$\begin{array}{lll}\text { Chairman } & \text { Prof. dr. ir. A.J. Mouthaan } & \text { University of Twente } \\ \begin{array}{l}\text { Promotor } \\ \text { Co-promotor }\end{array} & \begin{array}{l}\text { Prof.dr.ir. A. van den Berg } \\ \text { Dr. ir. W. Olthuis }\end{array} & \begin{array}{l}\text { University of Twente } \\ \text { University of Twente }\end{array} \\ \text { Members } & \begin{array}{ll}\text { Prof. dr. ir. U. Karst } \\ \text { Prof. dr. R.P.H. Bischoff }\end{array} & \begin{array}{l}\text { University of Münster } \\ \text { University of Groningen }\end{array} \\ & \begin{array}{l}\text { Prof. dr. S.G. Lemay } \\ \text { Prof. dr. J.G.E. Gardeniers }\end{array} & \text { University of Twente } \\ & \text { University of Twente } \\ & \text { Ar. U. Jurva } & \end{array}$

Odijk, Mathieu

Title:

ISBN:

Miniaturized electrochemical cells for applications in drug screening and protein cleavage

$\mathrm{PhD}$ thesis, University of Twente, The Netherlands

Publisher: Wöhrmann Print Service, Zutphen, The Netherlands

Cover design: Mathieu Odijk

The cover shows an iridium oxide thin-film with (intrinsic) stress sputtered in the Nordiko machine. This issue took one year of my $\mathrm{PhD}$ period. It was solved by the arrival of the TCOater sputtering machine.

Copyright (C) 2011 by Mathieu Odijk, Enschede, The Netherlands 


\title{
MINIATURIZED ELECTROCHEMICAL CELLS FOR APPLICATIONS IN DRUG SCREENING AND PROTEIN CLEAVAGE
}

\section{PROEFSCHRIFT}

\author{
ter verkrijging van \\ de graad van doctor aan de Universiteit Twente, \\ op gezag van de rector magnificus, \\ prof.dr. H. Brinksma, \\ volgens het besluit van het College voor Promoties \\ in het openbaar te verdedigen \\ op vrijdag 4 maart 2011 om 14:45 uur
}

door

Mathieu Odijk

Geboren op 23 juni 1981

te Enkhuizen 
Dit proefschrift is goedgekeurd door:

Promotor Prof. dr. ir. A. van den Berg

Co-promotor Dr. ir. W. Olthuis 


\section{Contents}

1 Aim and thesis outline 9

1.1 Project aim and description $\ldots \ldots \ldots \ldots$

1.2 Brief introduction to the Lab-on-Chip field . . . . . . . . . 11

1.3 Thesis outline . . . . . . . . . . . . . . 13

Bibliography ....................... 15

2 Applications and theory for on-chip electrochemistry 17

2.1 Introduction . . . . . . . . . . . . 18

2.2 Applications for on-chip electrochemistry . . . . . . . 19

2.2.1 Proteomics . . . . . . . . . . . . . . . 19

2.2.2 Drug screening . . . . . . . . . . . . 29

2.3 Electrochemistry . . . . . . . . . . 36

2.3.1 Theory of faradaic processes . . . . . . . 36

2.3.2 Design of miniaturized electrochemical cells . . . 49

2.3.3 Existing integrated cell designs $\ldots \ldots . \ldots 58$

2.4 Summary and outlook . . . . . . . . . . . . 66

Bibliography ..................... 70 
3 A chip for conversions in drug metabolism studies 79

3.1 Introduction . . . . . . . . . . . . 80

3.2 Theory and design considerations . . . . . . . . 82

3.3 Experimental ................... 85

3.3.1 Chip design and fabrication . . . . . . . 85

3.3.2 Cyclic voltammetry and conversion efficiency study 86

3.3.3 Amodiaquine study . . . . . . . . . . . 87

3.3.4 Instrumentation . . . . . . . . . . . . 87

3.3.5 Chemicals ................ 88

3.4 Results and discussion $\ldots \ldots \ldots . \ldots . \ldots 9$

3.4.1 Cyclic voltammetry measurements . . . . . . . 89

3.4.2 Conversion efficiency study . . . . . . . . . 91

3.4.3 Amodiaquine experiments . . . . . . . . . 99 91

3.5 Conclusions ........................ 93

Bibliography ......................... 95

4 Electrochemistry-on-chip for use in drug metabolism studies 97

4.1 Introduction . . . . . . . . . . . . . 98

4.2 Materials and methods . . . . . . . . . . . . 100

4.2.1 Chip fabrication ............ 100

4.2.2 Chemicals ................. 102

4.2 .3 Setup ......................... 102

4.2.4 Methods ................. 104 
4.3 Results and discussion . . . . . . . . . . . . 105

4.3.1 IrOx pH response . . . . . . . . . . . . . . 105

$4.3 .2 \quad$ IrOx stability . . . . . . . . . . . . . . 105

4.3.3 Procainamide metabolism study . . . . . . . . 106

4.4 Conclusion ...................... 113

Bibliography ....................... 114

5 Preliminary results of peptide cleavage on-chip 117

5.1 Introduction . . . . . . . . . . . . . 118

5.2 Theory . . . . . . . . . . . . . . . . . 119

5.3 Experimental ................... 121

5.3.1 Electrochemical cell on-chip . . . . . . . . . 121

5.3.2 Chemicals ................ 121

5.3 .3 Methods .................. 121

5.4 Results and discussion . . . . . . . . . . . . 123

5.5 Conclusion . . . . . . . . . . . . 125

Bibliography ...................... 126

6 Redox cycling and differential cyclic voltammetry 127

6.1 Introduction . . . . . . . . . . . . 128

6.2 Theory . . . . . . . . . . . . . . . . 130

6.2 .1 Governing equations . . . . . . . . . . 130

6.2 .2 Geometry .................. 132

6.2.3 Applied potentials . . . . . . . . . 133 
6.2 .4 Post-processing . . . . . . . . . . . . . . 134

6.3 Experimental . . . . . . . . . . . . . . 134

6.3.1 Sensor fabrication . . . . . . . . . . 134

6.3.2 Chemicals................ 136

6.3 .3 Methods ................. 136

6.4 Results and Discussion . . . . . . . . . . . . 136

6.4 .1 Simulation results . . . . . . . . . . 136

6.4.2 Experimental results . . . . . . . . . . . 142

6.4.3 Model and experimental agreement . . . . . . . 143

6.5 Conclusion . . . . . . . . . . . . . 146

Bibliography ..................... 147

7 Conclusions and outlook $\quad 149$

7.1 Conclusions . . . . . . . . . . . . . . . . . . . 149

7.2 Outlook ...................... 150

Bibliography ....................... 152

A Appendix

A.1 Process document for the EC cell on-chip . . . . . . . . 154

A.1.1 Introduction . . . . . . . . . . 154

A.1.2 Mask layout . . . . . . . . . . . . . . 154

A.1.3 Processing steps . . . . . . . . . . . . 159

A.2 IrOx sputtering and adhesion study . . . . . . . . 166

A.3 List of standard amino acids $\ldots \ldots \ldots$ 
Abstract

Samenvatting

List of publications

175

Dankwoord 



\section{Aim and thesis outline}

In this first chapter, background and aims of a project to develop miniaturized electrochemical cells are given. The position of this work within the Lab-onChip field is discussed. At the end of this chapter an outline for the rest of this thesis is given. 


\subsection{Project aim and description}

The work presented in this thesis is the result of the 'LC / on-chip electrochemistry / mass spectrometry in (bio)analytical chemistry, drug metabolism and proteomics' project. It is funded (mainly) by the dutch science and technology foundation, called 'Stichting Technische Wetenschappen' (STW). In short, this project aims at the development of miniaturized electrochemical cells used in two main application areas. In the first application area, electrochemistry is used to study the metabolism of drugs. In the second application area, electrochemistry is used to provide an alternative method for protein modification. Protein modification is frequently used in the field of proteomics, where complex mixtures of proteins of a single cell or organism are studied. The project is a multidisciplinary, collaborative effort of three groups, spread across three universities.

The first group involved is the Institute of Inorganic and Analytical Chemistry, headed by Prof. Uwe Karst and located in Münster - Germany. The main focus of this group within this project is to study discovery, metabolism, and development of drugs. This group is therefore mainly involved with the first application introduced in the previous paragraph. Just before the start of this project, Prof. Karst moved from the University of Enschede to Münster, which makes this project unique in the sense that STW funded projects usually involve groups from dutch universities only. Part of the work presented in this thesis is therefore funded by the Deutsche Forschungsgemeinschaft (DFG), the German counterpart of STW.

The second group contributing to this project is the Analytical Biochemistry group from the Centre of Pharmacy of the University of Groningen, headed by Prof. Rainer Bischoff. Also involved and located within the same centre is the Mass Spectrometry Core Facility, headed by Dr. Andries Bruins and Dr. Hjalmar Permentier. The main focus of this group lies within the development of an instrumental method for protein and peptide analysis. A second research area is the development of novel methods using electrochemistry and reactive intermediates to mimic the metabolism of drugs.

In contrast to the two analytical chemistry groups presented so far, the third group has expertise in the development of Lab-on-Chip devices. The BIOS, Lab-on-a-Chip Group from the University of Twente. headed by Prof. Albert van den Berg. BIOS has four focus areas, of which one is aimed at 
electrochemical systems and applications. The electrochemical focus area is managed by Dr. Wouter Olthuis. The role of this group within this project is to develop miniaturized electrochemical cells, specifically aimed at the two previously discussed applications. This thesis is the direct result of this effort. The title of this thesis is therefore: 'Miniaturized electrochemical cells for applications in drug screening and protein cleavage'.

\subsection{Brief introduction to the Lab-on-Chip field}

The term Lab-on-Chip (LOC) refers to a device that integrates one or more laboratory functions on a single chip. In general, it involves handling of fluids on the microscale using small microchannels and (possibly) pumps or valves. The term microfluidics is therefore frequently used in conjunction with LOC. One of the pioneers in the field are Manz et al. [1,2], introducing the concept of miniaturized total analysis systems. This term was later referred to as micro total analysis systems ( $\mu$ TAS) [3]. While the term $\mu$ TAS mainly focuses on analysis, LOC refers to a wider field including e.g. chemical synthesis or cell culture. Microfluidics can be regarded as the enabling technology for the LOC field.

An overview of current technology for microfluidic is presented in a review by George Whitesides [4]. In this review, four parents of the field of microfluidics are distinguished: molecular analysis, biodefence, molecular biology and microelectronics. Separation methods like chromatography or electrophoresis in capillary form have revolutionized molecular analysis. Therefore, microfluidics provides possibilities in molecular analysis to simultaneously achieve high sensitivities and high resolution while using only small amounts of sample. The field of molecular biology motivated microfluidics development by the genomics project in the 1980s, followed by the need for high-throughput DNA sequencing in forensics. The US defence advanced research project agency (DARPA) gave a main stimulus to the microfluidic community by a series of programmes in the 1990s aimed at developing fielddeployable microfluidics as detectors for chemical and biological threats. Initially LOC technology was mainly based on experise developed in MEMS and IC fabrication technology. Over the last decade however, the field of LOC showed many efforts in the development of novel, often polymer based fabrication methods. In particular PDMS is a very popular material used in LOC, because it provides the possibility for rapid prototyping. 
LOC is a very multidisciplinary field, since applications for LOC devices can be found in single-cell analysis [5, 6], chemical synthesis [7], DNA analysis [8], separation methods like electrophoresis [9] or chromatography [10], and detection methods based on electrochemistry [11], optics [12], nuclear magnetic resonance (NMR) [13] or mass spectrometry [14]. The examples described so far include research conducted within the BIOS group (or the MCS group, a former part of BIOS) over the period of 2004-2009 alone, many more examples can be found in literature.

The popularity for LOC, can be explained by several unique beneficial properties provided by miniaturization.

- Due to the drastic increase in surface to volume ratio in micro- or nanofluidic channels, unique phenomena are observed. E.g. in nanofluidic channels, silicon walls can cause a significant change in $\mathrm{pH}$, due to titration of the solution by silanol groups from silicon oxide surfaces [15].

- LOC technology also promises low-cost, disposable devices, which is especially beneficial to prevent costly or labor intensive cleaning or sterilization procedures in e.g. medical applications [16].

- In general LOC devices consume less reagent, as a result the waste production is also lower. One important aspect especially in chemical research areas is the increased safety provided by LOCs. The risks involved when working with reactions that might produce toxic vapors or explosions are much lower, since less analyte is available. Furthermore, the risk for working at high pressures is much smaller since an exploded LOC causes much less damage [7].

- LOC technology can provide better control over the process occurring inside the device. Inside a LOC, heat transfer is mainly driven by conduction through the substrate material. Due to the decreased dimensions less heat capacity is available, thus providing the advantage of a faster response of the total system. This benefit is used e.g. to speed-up the process of the polymerase chain reaction ${ }^{1}$ by performing it on-chip [17].

${ }^{1} \mathrm{PCR}$ is a method to multiply parts of DNA by chemical means. It involves several temperature steps to control the process. 
- Another feature provided by LOC technology is portability. This is especially important for point-of-care diagnostics or in-the-field measurements. However, it also puts demands on surrounding equipment like pumps, power supplies and readout electronics [16].

- Microfluidics usually deals with small sample volumes. For production of larger volumes of chemical products, (massive) parallelization remains possible. Analogous to the large scale integration of many transistors in computer memory and processors, parallelization of microfluidic devices keeps the previously mentioned advantages of LOC technology while acquiring sufficient product [18].

In this project, the aim is to use LOC technology to provide high electrochemical conversion efficiencies. One of the main aspects that affect the rate of conversion at the electrode is the mass transport of ions towards the electrode surface. Since the surface to volume ratio is greatly enhanced in a microfluidic channel, LOC seems to provide clear benefits. Moreover, one of the applications lies in drug screening. In the pre-clinical phase, the production of trial compounds is often a costly process due to product generation and isolation. A LOC device might reduce costs due to a smaller analyte consumption. Finally, a disposable LOC device can reduce even more costs, because it prevents the need for cleaning of the electrochemical cell.

\subsection{Thesis outline}

As the field of Lab-on-Chip is very multidisciplinary, first a basic introduction is given for those not familiar to the two main application areas of proteomics and drug screening in chapter 2. It continues with a more detailed introduction into electrochemical processes applicable to conversion reactions. At the end of chapter 2, the design of miniaturized electrochemical cells is discussed, followed by a literature review of existing cell designs.

In chapter 3, the design of a novel miniaturized electrochemical cell is presented. This cell is characterized by cyclic voltammetry. Next, results are presented that study the conversion efficiency of this cell for small and fast reacting ions using UV-vis spectroscopy as detection method. Chapter 3 ends with demonstrating the feasibility of using this chip for drug screening by a comparison with commercially available cells. 
Chapter 4 presents an improved version of the miniaturized cell using iridium oxide as pseudo-reference electrode. The $\mathrm{pH}$ dependence and potential stability of this pseudo-reference electrode is studied by $\mathrm{pH}$ and potentiometric measurements. Chapter 4 also demonstrates the use of this improved miniaturized cell by a comparison between the standard method of liver cell microsomal incubations and direct electrochemical oxidation as a way to mimic the oxidative metabolism catalyzed by the cytochrome P450 enzyme family.

Preliminary results of electrochemical protein cleavage are presented in chapter 5 to demonstrate that this approach is feasible using the miniaturized electrochemical cell. The main message in chapter 5 is a suggestion for several improvements for further research to make the best out of this application.

Chapter 6 switches gears, in the sense that it is more focused towards detection than conversion. In this chapter, a novel measurement method is presented that uses the redox cycling effect for more sensitive and selective measurements of (reversible) redox active compounds. This method is demonstrated both theoretically using computer simulation models, and experimentally by measurements using interdigitated array electrodes.

Finally, the main findings of this thesis are summarized in chapter 7. 


\section{Bibliography}

[1] A. Manz, N. Graber, and H.M. Widmer. Miniaturized total chemical analysis systems: A novel concept for chemical sensing. Sensors and Actuators B: Chemical, 1(1-6):244 - 248, 1990.

[2] A. Manz and H. Becker, editors. Microsystem Technology in Chemistry and Life Sciences. Number ISBN 3-540-65555-7. Springer-Verlag, 1999.

[3] A. van den Berg and T.S.J. Lammerink. Micro total analysis systems: Microfluidic aspects, integration concept and applications. In Manz and Becker [2].

[4] George M. Whitesides. The origins and the future of microfluidics. Nature, 442:368-373, 2006.

[5] Jurjen Emmelkamp. An integrated micro bi-directional dosing system for single cell analysis on-chip. PhD thesis, University of Twente, 2007.

[6] Floor Wolbers. Apoptosis chip for drug screening. $\mathrm{PhD}$ thesis, University of Twente, 2007.

[7] Roald Tiggelaar. Silicon-technology based microreactors for hightemperature heterogeneous partial oxidation reactions. $\mathrm{PhD}$ thesis, University of Twente, 2004.

[8] Georgette Salieb-Beugelaar. Electrokinetic Transport of DNA in Nanoslits. PhD thesis, University of Twente, 2009.

[9] D. Kohlheyer. Microfluidic Free-Flow Electrophoresis for Proteomicson-a-Chip. PhD thesis, University of Twente, 2008.

[10] W. de Malsche. Solving advanced micromachining problems for ultrarapid and ultra-high resolution on-chip liquid chromatography. $\mathrm{PhD}$ thesis, University of Twente, 2008.

[11] Erik Krommenhoek. Integrated sensor array for on-line monitoring micro bioreactors. PhD thesis, University of Twente, 2007.

[12] Bianca Beusink. Label-free biomolecular interaction sensing on microarrays using surface plasmon resonance imaging. $\mathrm{PhD}$ thesis, University of Twente, 2009.

[13] Jacob Bart. Stripline-based microfluidic devices for high-resolution NMR spectroscopy. PhD thesis, University of Twente, 2009.

[14] Wojciech Piotr Bula. Microfluidic devices for kinetic studies of chemical reactions. $\mathrm{PhD}$ thesis, University of Twente, 2009.

[15] Kjeld G. H. Janssen, Hanh T. Hoang, Jan Floris, Jeroen de Vries, 
Niels R. Tas, Jan C. T. Eijkel, and Thomas Hankemeier. Solution titration by wall deprotonation during capillary filling of silicon oxide nanochannels. Analytical Chemistry, 80(21):8095-8101, 2008. PMID: 18826247.

[16] Arjan Floris, Steven Staal, Stefan Lenk, Erik Staijen, Dietrich Kohlheyer, Jan Eijkel, and Albert van den Berg. A prefilled, ready-touse electrophoresis based lab-on-a-chip device for monitoring lithium in blood. Lab on Chip, 10:1799-1806, 2010.

[17] Jian Liu, Carl Hansen, and Stephen R. Quake. Solving the "worldto-chip" interface problem with a microfluidic matrix. Anal Chem, 75:4718-4723, 2003.

[18] Todd Thorsen, Sebastian J. Maerkl, and Stephen R. Quake. Microfluidic large-scale integration. Science, 298(5593):580-584, 2002. 


\section{Applications and theory for on-chip electrochemistry}

In this chapter, the two applications that are the topic of this thesis are introduced. One application is related to drug screening, the other application involves electrochemical protein digestion in the field of proteomics. First a general overview of both topics is given. Next, the role of electrochemistry in these fields is explained. Subsequently, an overview is given of the current status in literature of lab-on-chip technology used in these fields.

The second part of this chapter gives an introduction into the field of electrochemistry, with a special focus towards electrochemical conversion reactions at electrode-liquid interfaces. After this introduction, the design of miniaturized electrochemical cells is discussed. In the end of this chapter, an overview of currently published electrochemical cell designs is presented. 


\subsection{Introduction}

Nowadays, the laboratory of an analytical chemist is packed with a lot of (expensive) tools like mass spectrometers, high performance liquid chromatography (HPLC) pumps, HPLC columns, UV spectrometers and more. In that light, electrochemistry is often regarded as one of many techniques to gain more insight in a specific chemical process. Two of these processes are topic of this thesis.

Drug screening involves the search for new drugs, or the search for more insight in (side-)effects of existing drugs. The way the human body handles drugs is a complicated process. Years of in vitro and animal model testing in the lab are conducted, before new drugs are tested on human subjects. Electrochemistry can be used as tool to mimic the way the human liver metabolizes many drugs. It provides the advantage of a fast and cheap method to screen for possible toxic byproducts or how inactive drugs are metabolized into active products.

The other process involves peptide cleavage. Peptide mapping is a widely used method in proteomics for its ability to identify unknown proteins. Proteins are cleaved into peptides and subsequently analyzed by mass spectrometry (MS). The resulting set of peptides is compared to a protein database to identify the unknown protein. This method relies on a reliable cleavage method, usually done by enzymatic digestion using trypsin. Trypsin shows specific cleavage in proteins at the carboxylic side of the amino acids arginine or lysine. Electrochemistry can be used as tool to provide an alternative, complementary cleavage method specific to peptide bonds next to tyrosine or tryptophan amino acids.

Miniaturized electrochemical cells in a lab-on-chip (LOC) format can provide specific advantages. Electrochemical reaction rates are often limited by mass transport of ionic species. Due to the small size, a LOC device might offer specific advantages to achieve a high conversion efficiency of introduced products. Also, volumes are small, giving the possibility to work with small amounts of products. Moreover, LOC devices can be used as low-cost disposables if produced in sufficient quantities. Disposable devices also circumvent the need for extensive cleaning procedures of the electrodes after use. The goal of this thesis is to design such a miniaturized cell. However, these cells 
need to be well designed and reliable to be accepted as tool in the world of analytical chemistry.

In the rest of this chapter, a basic, general introduction is given into the two applications presented in this thesis. Next, some fundamentals of electrochemistry are discussed with a focus towards faradaic processes. Subsequently, this basic electrochemistry knowledge is applied to specific problems faced when designing a miniaturized electrochemical cell. Finally, a literature overview is given on the topic of (miniaturized) electrochemical (EC) cells.

\subsection{Applications for on-chip electrochemistry}

Two applications for on-chip electrochemistry are introduced. First a general introduction in proteomics is given, which will focus towards protein cleavage at the end. Next, the field of drug screening is introduced, followed by a discussion of the role of the electrochemically active enzyme cytochrome P450 in drug metabolism processes. Finally, direct electrochemical oxidation at electrodes is compared to electrochemical reactions catalyzed by cytochrome P450.

\subsubsection{Proteomics}

This section (2.2.1) is based on Mishra [1], Liebler [2] and [3].

Proteomics is the science in which the proteome of an organism is studied. The term proteome is introduced by Marc Wilkins in 1994 [4]. One way to explain what proteomics is about, is to use the concept of the central dogma of molecular biology [5].

\subsubsection{Central dogma}

The concept of the central dogma describes why DNA is responsible for the enormous amounts of different proteins produced by an organism [5]. Although the central dogma is already challenged and somewhat outdated due to many recent discoveries, it can still be used to give a short, simplified description of the general process from DNA to protein production [6].

DNA can be regarded as the blueprint for the proteins produced by an organism. The general way in which this process takes place is illustrated in 
figure 2.1. In figure 2.1 a cell is indicated by the large yellow ellipse, with its nucleus indicated in blue by the large blue ellipse. Inside the nucleus DNA is present. The general pathway from DNA to protein is a 3-step series of events.

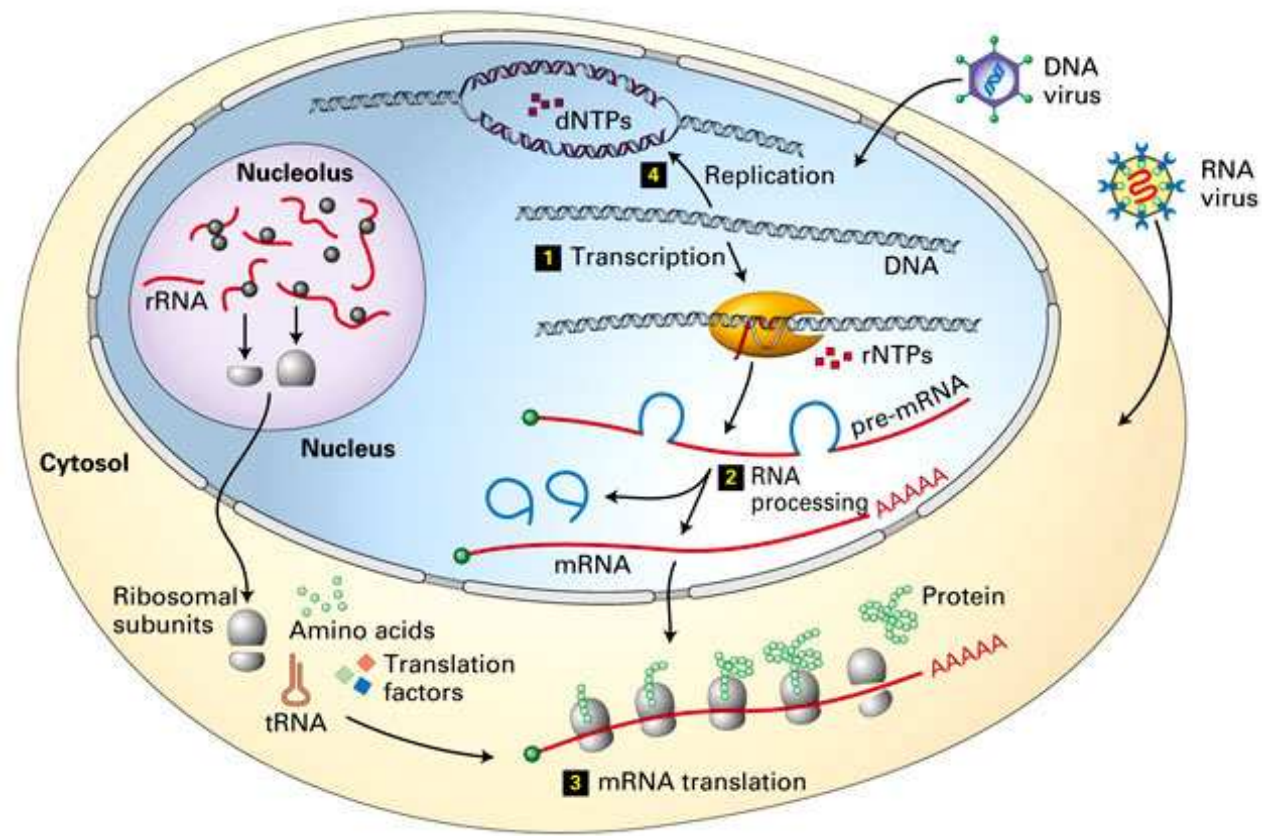

Figure 2.1: General mechanism of DNA to protein translation inside a cell [7].

In the first step, a small part of DNA (gene) is transcribed (copied) into pre-mRNA by an enzyme called RNA polymerase. Next, the pre-mRNA is processed such that both ends are capped. Moreover, non-coding parts (called introns, blue lines) are removed from the pre-mRNA, such that the coding parts (called exons, red lines) remain. This intron removal process, called splicing is carried out by the spliceosome. Splicing of pre-mRNA is one of the things that sets the eukaryotic organisms apart from the prokaryotic organisms ${ }^{1}$. After processing the mRNA is transported out of the nucleus.

In a third step, the mRNA is translated into proteins by the ribosomal units, with the help of tRNA. A set of 3 base pairs (called codon) present in the

${ }^{1}$ An eukaryote is an organism of which the cells contain a nucleus, e.g like humans. A prokaryote is an organism without a nucleus, e.g. bacteria and archaea. 
mRNA codes for one specific amino acid of the twenty amino acids available (see A.3). This translation of codons into amino acids is conducted by tRNA. A long chain of amino acids is formed by linking the carboxyl group of one amino acid with the amino group of another amino acid; this link is called a peptide bond.

Splicing is not always carried out in exactly the same fashion. Therefore the resulting end product (mRNA) is not always the same from its parent gene. Alternate splicing can produce a whole range of different mRNAs. E.g. in humans it is estimated that 23000 genes are responsible for 90000 different proteins, excluding post translational modifications which result in even higher estimated amount of 500000 different proteins. In contrast; in prokaryotes almost $100 \%$ of the genes encode one gene per protein [1]. So far, the steps from DNA to protein are described as a one-way process. Recent discoveries indicate that this simplified pathway is in fact much more complicated and therefore beyond the scope of this thesis [6].

In earlier days, before the human genome project, some scientist believed that humans may have 100000 genes or more. This believe was a result of the one-gene-one-protein theory, which is true for most proteins in prokaryotes. Scientist hoped that knowing the genome of an organism would lead to knowledge of the entire proteome. This was one of the motivations to map entire genomes with the field of genomics gaining lots of interest. Due to the discovery of all the different splicing mechanisms, post translational protein modifications and RNA silencing, scientists realized that knowledge of the genome alone was not enough. In that respect, the field of proteomics can be regarded as the next step to genomics.

\subsubsection{Proteins}

In general, proteins can be regarded as the workhorses of living cells. Proteins are involved in almost all processes occurring in living organisms. In table 2.1 a list of protein functions is included with some example proteins.

Proteins and peptides are made of a chain of amino acids ${ }^{2}$. There are 20 different amino acids that occur in living organisms (see A.3). The primary

\footnotetext{
${ }^{2}$ The difference between a protein and a peptide is the number of amino acids involved. In general a protein is regarded as a chain with 50 or more amino acids.
} 
Table 2.1: Different protein functions and some examples.

\begin{tabular}{ll}
\hline Function & Protein examples \\
\hline \hline Catalyst & $\begin{array}{l}\text { Enzymes like e.g. RNA poly- } \\
\text { merase }\end{array}$ \\
Transport & $\begin{array}{l}\text { Hemoglobin (oxygen), albumin } \\
\text { (fatty acids) }\end{array}$ \\
Structure & Collagen (bone protein) \\
Cellular skeleton & Actin, fibrinoactin \\
Hormone & Insulin \\
Antibody & Immunoglobulins \\
Antigens and allergens & Bacterial and viral proteins \\
Mobility / muscle movement & Myosin \\
Receptors & Rhodopsin (light detection) \\
Communication / signaling & Transduction proteins, junction \\
& proteins (e.g. GTPases) \\
Storage & ovalbumin and casein (energy) \\
\hline
\end{tabular}

structure of a protein refers to the sequence of amino acids present within one protein, as depicted in figure 2.2. Hydrogen bonds can form between the amino acids of a single chain, resulting in folding of the chain. Two different ways of folding are known, the $\alpha$-helix and the pleated $(\beta)$ sheet. This folding is referred to as the secondary structure (fig. 2.2). The two forms of folding can both be present within a single protein. Resulting in a complex 3D structure, called the tertiary structure. Finally, multiple proteins can entangle to form a quaternary structure as shown in figure 2.2.

\subsubsection{Protein separation methods}

Measurements in the field of proteomics usually start with a (complex) mixture of proteins or peptides. One of the crucial steps in identifying these proteins and peptides is a good separation method. Several methods exist, of which electrophoresis and chromatography are the most used in this field. Each of the two methods named here can be subdivided into several submethods. E.g. electrophoresis, can be subdivided into 2D gel electrophoresis, capillary electrophoresis, free-flow electrophoresis and so on. Chromatography has an even longer list of specific sub-methods. Each sub-method has its 
(a) Primary structure

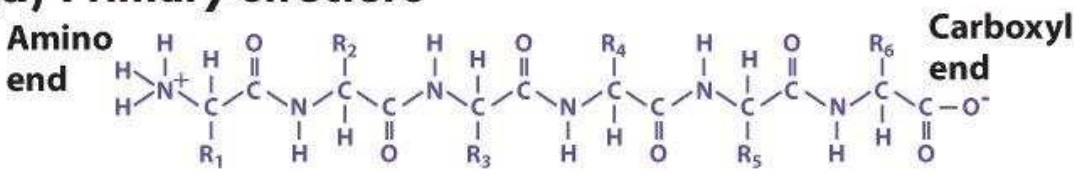

(b) Secondary structure

Hydrogen bonds between

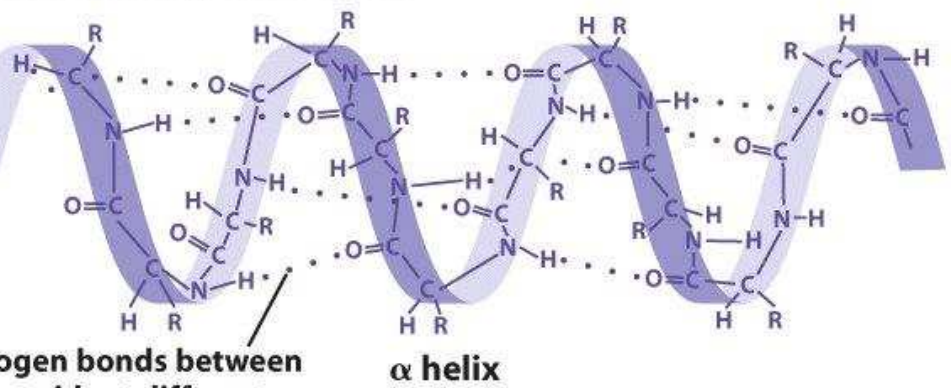

amino acids at different locations in polypeptide chain

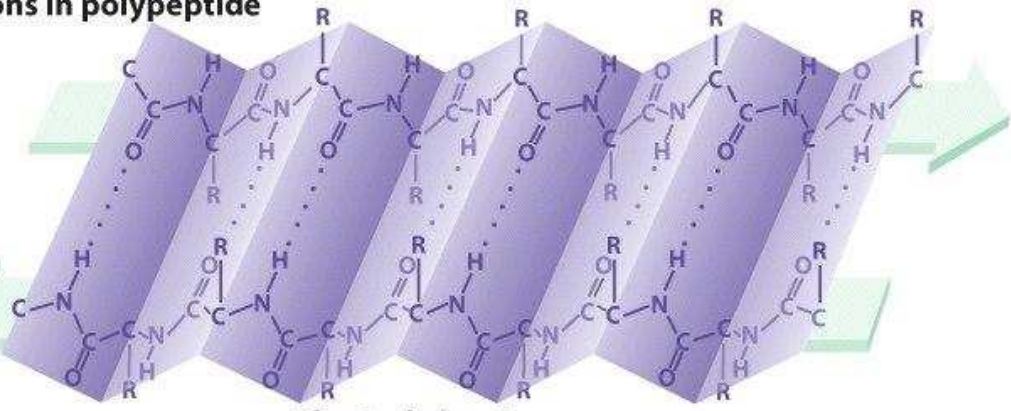

Pleated sheet

(c) Tertiary structure

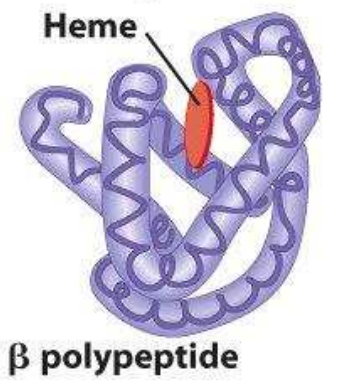

(d) Quaternary structure

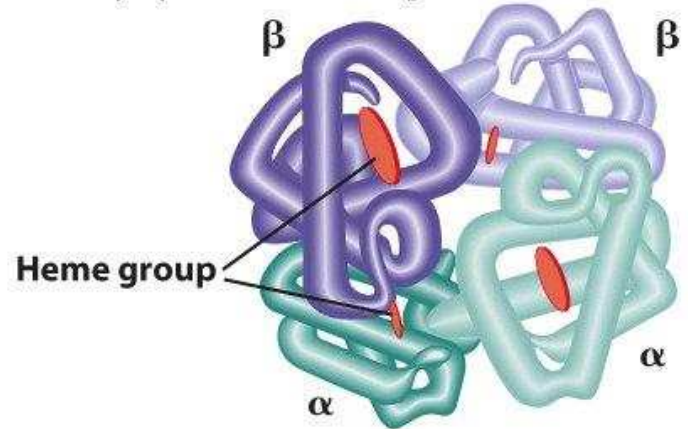

Figure 2.2: Explanation of the different protein structure categories (see text for details) [8]. 
own working principle and specific uses. It is beyond the scope of this thesis to discuss each method in depth. Therefore only the method of reversed phase high performance liquid chromatography (RP-HPLC) is discussed here for its use in chapter 3 and 4.

The basic idea of each separation method is to take advantage of the differentiation in physical properties between the target molecules. In reversed phase HPLC, separation will occur due to differences in hydrophobicity of the different proteins [9]. A column is filled with a stationary phase, like e.g. silica beads. These beads are usually coated with a non-polar compound like e.g. silyl ethers containing non-polar alkyl groups (C8 or C18) to get a hydrophobic surface. The working principle is explained in figure 2.3. Proteins will most likely contain a mix of hydrophobic and hydrophilic amino acids. Only those with a net hydrophobicity will be able to bind to the hydrophobic surface. The net hydrophilic proteins will wash through the column, because they have a greater affinity with the hydrophilic buffer. By changing the buffer composition, the hydrophobicity can be modified. This will result in desorption of the more hydrophobic proteins. By using a slow gradient in the buffer concentration (e.g. from $100 \%$ water to $100 \%$ acetonitrile or another organic solvent) proteins are desorbed and washed out of the column at different points in time and therefore separated based on their difference in hydrophobicity.

The separation efficiency is largely dependent on the surface area of the stationary phase. Therefore usually porous silica beads are used in reversed phase chromatography. In general, smaller beads will also give a higher surface area. However, the pressure required to push the liquid through will also increase rapidly [10]. In (ultra) high performance liquid chromatography, the used liquid pumps are able to deliver stable flow rates at high (above 10MPa) pressures.

\subsubsection{Protein identification methods}

Different methods exist to characterize either the primary or 3D structure of a protein. The study of 3D-structures (e.g. by X-ray crystallography, X-ray diffraction, NMR) is beyond the scope of this thesis. Several methods exist for the identification of the primary structure, amongst those methods are DNA-sequence based identification, Edman degradation and mass spectrometric identification. 


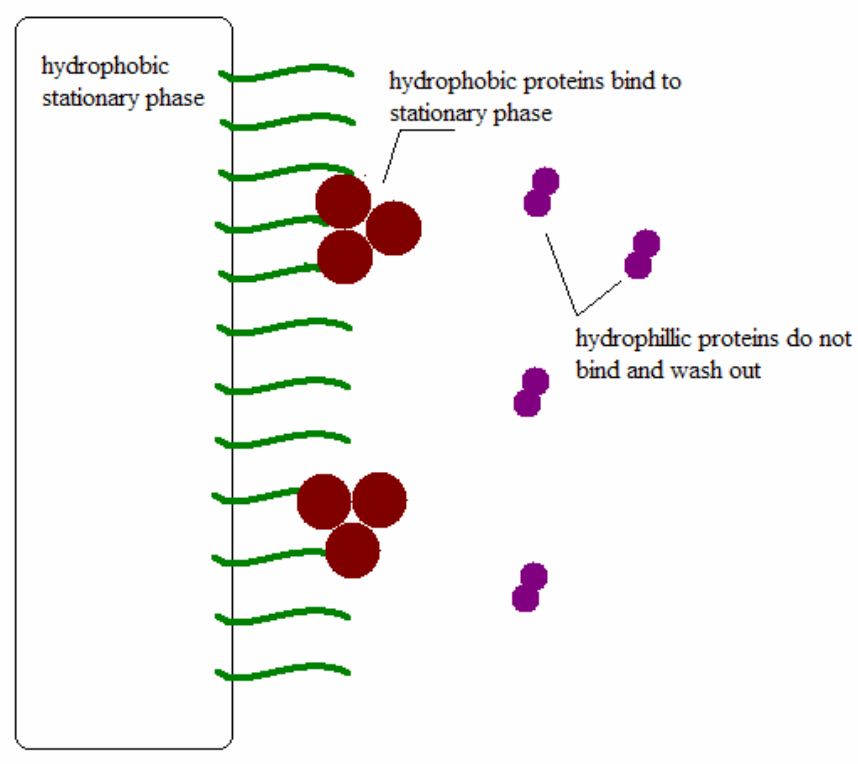

Figure 2.3: Illustration of the basic working principle of reversed phase liquid chromatography [8].

DNA-sequence based identification infers the amino acid sequence of a protein from the nucleotide sequence of the DNA. This method has its limitations; especially in eukaryotic cells the translation from DNA to protein is not easy to predict due to e.g. the presence of intron sequences.

In Edman degradation, proteins are cleaved one amino acid at a time from the $\mathrm{N}$-terminus of the peptide. In a first step, the N-terminal amino acid reacts with phenylisothiocyanate under mild alkylic conditions. In a second step the intermediate $\mathrm{N}$-terminal product is removed from the rest of the protein under acidic conditions. In a final step, the removed $\mathrm{N}$-terminal product is isolated from the protein using appropriate solvents and treated with acid to form a stabilized product which can be analyzed further. The major drawback of this method is the limited length of the peptide under investigation. In practice only peptides with a sequence of up to 40 amino acids can be analyzed accurately [11]. Moreover, many proteins contain blocked N-terminal groups which cannot react with the phenylisothiocyanate required for the degradation [1]. Also, this method is limited to one protein at a time, while proteomics aims to gain information of several proteins at the same time. 
Probably the most used method nowadays is mass spectrometric detection. Mass spectrometry (MS) was invented around 1900 by J.J. Thomson [12]. For this invention he received the Nobel prize in physics in 1906. In MS, charged molecules are detected based on their mass-to-charge ratio. Various different designs of mass spectrometers exist, but all have in common that charged molecules are manipulated under influence of electric fields. The trajectory of heavier molecules or molecules with a lower charge will change less due to the electric field than that of lighter or more charged molecules.

Before detection, molecules need to be charged or ionized and introduced into the mass spectrometer. Several ionization methods exist such as atmospheric pressure chemical ionization (APCI), matrix assisted laser desorption ionization (MALDI), electron or chemical ionization (EI or CI) and electrospray ionization (ESI). Only ESI is discussed here in more depth, because of its use in chapter 3 and 4.

ESI is invented by J.B. Fenn [13], for which he received the Nobel prize in chemistry (partially) in 2002. Electrospray ionization is regarded as a soft ionization method, since the molecules being ionized do not fall apart or break-up during the process. It is particularly useful when analyzing large molecules like proteins. The working principle is illustrated in figure 2.4.

In ESI, liquids are introduced into the MS via a small (metal) capillary. A high potential (1-5 kV typically) is applied between this capillary and the opening plate of the MS. In the figure, positive ions will be attracted towards the more negative counter electrode formed around the opening of the MS. Just before the spray initiates, the liquid will form a conical shaped tip, which is referred to as the Taylor cone. Droplets will leave the tip and travel towards the opening. During this travel solvent will evaporate, effectively shrinking the droplet. Upon a certain limit (called Rayleigh limit) the droplet will become unstable due to increasing repelling electrical forces caused by the decreasing distances of the charges inside the droplet. The droplet will deform and emit charged jets in a process known as Coulomb fission [14, 15].

One of the main advantages of ESI is that the ionization efficiency will increase if smaller capillaries are used. ESI is therefore an ideal ionization method for microfluidics, as will be discussed in the next subsection [16].

In general, the protein identification using MS techniques can be divided into two approaches. In top-down analysis, whole proteins are injected into the 


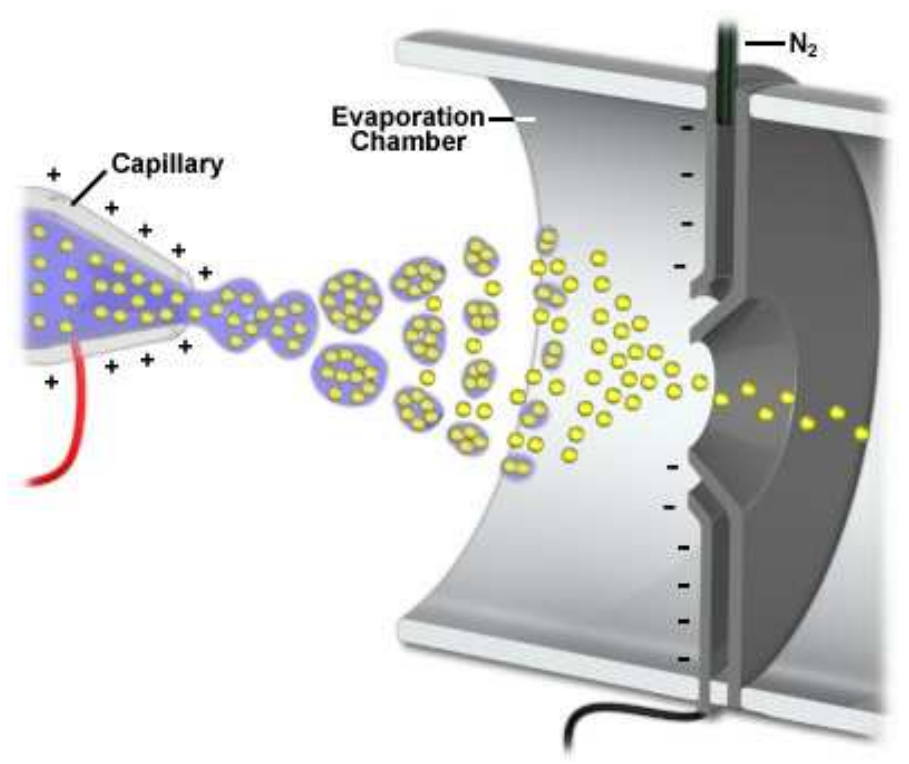

Figure 2.4: Illustration of the working principle of an electrospray ionization interface.

MS. During this process, the protein will partially fragment. The resulting fragmentation pattern combined with the mass of the whole protein is compared to a database of known proteins for identification [17]. In the bottom-up approach, proteins are first cleaved into smaller fragments e.g. by enzymatic digestion using trypsin. Again, the data obtained in this way is compared to a database to identify the protein. Essential for this method to work is a good (high yield) and reproducible cleavage method, which explains the popularity for tryptic digestion. Trypsin shows specific cleavage of peptide bonds on the carboxylic side between arginine or lysine with another amino acid, except if the other amino acid is proline $[1,2]$.

In chapter 5, an alternative method to tryptic digestion is proposed. This method is based on electrochemical cleavage of proteins, which is shown to be specific for bonds on the carboxylic side of tyrosine or tryptophan with another amino acid [18-21]. More details on the mechanism of this cleavage is discussed in chapter 5 . 


\subsubsection{Lab-on-Chip in proteomics}

The measurement challenges faced in the field of proteomics in terms of complexity, sensitivity and variations in molecule properties (e.g. isoelectric point and size) are tremendous. Lab-on-Chip technology provides many advantages which can help to address these challenges. In the last years, several reviews have been published discussing the application of Lab-on-Chip technology in the field of proteomics.

The extensive review of Lion, Girault and coworkers [22], discusses the application of microfluidics in the field of proteomics. It gives a very broad overview on micro-fabrication methods, separation methods, microfluidic enzymatic assays and immunoassays and the coupling of microfluidics to mass spectrometry. A recent review of the same group by Prudent and Girault [23], goes into more detail on the latter topic. This review discusses electrospray emitters extended with specific functions like separations or electrochemical conversions. A review of Koster and Verpoorte [24] presents a more general overview of electrospray emitters, integrated in lab-on-chip systems. Finally, a review of Freire and Wheeler [25] stresses that a successful marriage between proteomics and the microfluidic community is not yet fully achieved. However, very promising steps have been made in microfluidic systems to meet the various demands for the proteomic work-flow.

The mass spectrometer itself is also subject to miniaturization, although the developed machines cannot compete with macroscale systems [26-28]. So far, a miniaturized MS is only useful for specific applications where portability is most important.

Some recent state-of-the-art microfluidic applications for proteomics are highlighted here to give an impression. The first device highlighted in figure 2.5a is included for its system integration. It is a device fabricated by Xie et al. [29] and includes three electrochemical pumps, a mixing chamber, a separation column, and an ESI nozzle. The device was used to perform LC-MS analysis of a mixture of peptides from the tryptic digestion of bovine serum albumin. The device is made from a silicon substrate. SU-8 and parylene are used to provide microfluidic channels, the cavities for the separation column and mixer, and the ESI nozzle. The separation column is filled with $3 \mu \mathrm{m} \mathrm{C}-18$ coated beads. After this filling, the chip is sealed at the top by a 
polyetherimide sheet providing fluidic inlets and outlets and reservoirs for the electrochemical pumps. Gas formation by electrolysis at platinum electrodes forces liquid into the chip, providing a pumping mechanism. Using three of these pumps, sample can be injected and a gradient of 0 to $95 \%$ between the contents of pump A to B is applied. The pumps contain a mixture of 95/5/0.1 (pump A) and 40/60/0.1 (pump B) water/methanol/formic acid. Since water is required for the electrolysis, the mixture is limited to contain at least $40 \%$ of water, which is one of the weak points of this device. The packaging of the column and reproducible filling of the pumping reservoirs is the other drawback of this chip.

Liuni et al. [30] fabricated a protein digestion on-chip device with integrated ESI nozzle, which is shown in figure 2.5b. The design and fabrication of this chip is straightforward and comprises laser ablation of a PMMA sheet to form a shallow reactor chamber and microfluidic channels. The chamber is packed with commercially available pepsin-agarose beads. A cover sheet of PMMA is clamped on the laser ablated sheet with a nitrile rubber gasket in between to seal the microfluidic channels. The chip is used for tryptic digestion of myoglobin (horse heart), bovine serum albumin, and ubiquitin. The overall digestion efficiency was $99 \%$ to $88 \%$ at flow rates between 50 and $10 \mu \mathrm{L} / \mathrm{min}$. (residence time 4 to $15 \mathrm{~s}$ ), depending on the size of the protein.

The examples discussed so far are showing a proof-of-concept device. Unfortunately, many microfluidic devices do not make it into commercialized products. The last example however, shown in figure $2.5 \mathrm{c}$, is commercially available from the company Agilent. It is a microfluidic chip based cartridge, containing a separation column, an enrichment column, and an ESI sprayer tip. Moreover, it contains the seal of an off-chip rotary valve, which provides switching of fluids on-chip with low dead volumes.

\subsubsection{Drug screening}

In the past decades, costs to develop a new drug, accepted by the FDA ${ }^{3}$, have been growing exponentially to an estimate of 802 to 1318 million dollar [32]. FDA approval is important, since the US is by far the largest of all international markets. The typical work-flow of drug screening starts with years of extensive pre-clinical research [33].

\footnotetext{
${ }^{3}$ US Food and Drug Administration
} 


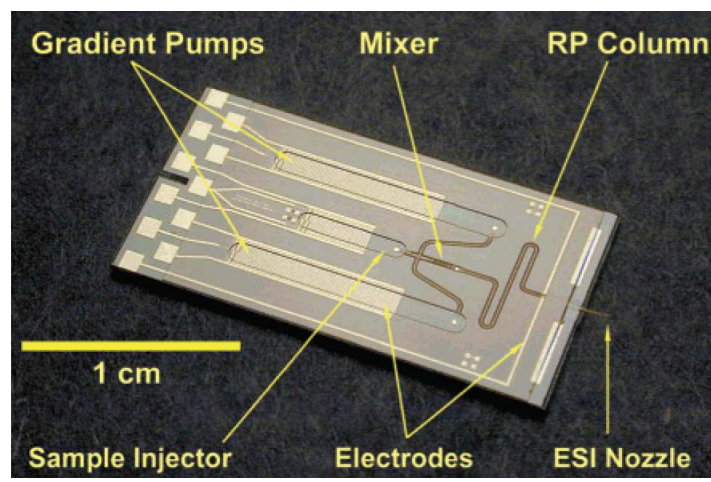

(a) Integrated pumps, LC column and ESI interface in a single device [29].
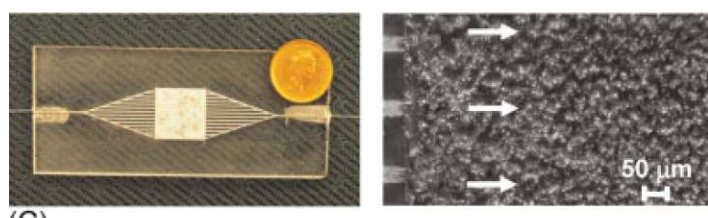

(C)

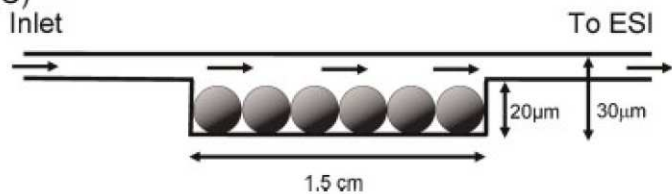

(b) Proteolysis on-chip device with ESI device [30].

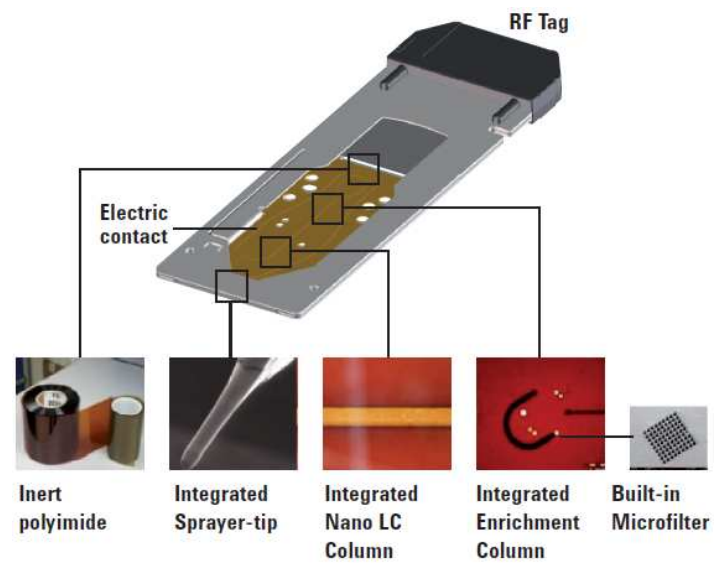

(c) Commercially available device from Agilent, with LC \& enrichment column and ESI tip [31].

Figure 2.5: Highlights of some state-of-the-art microfluidic devices used in proteomics. 
In this pre-clinical research, often large numbers of molecules are tested with the goal of identifying the most promising candidate. These in vitro tests often look at binding to receptors, effects on enzyme activities, toxic effects, or other in vitro parameters. For these in vitro tests, only small amounts of the molecule are required. The next step in pre-clinical trials involves larger volumes of promising candidates, which are produced for animal model testing. In the end, only one or very few compounds are selected for further clinical trials. These clinical trials are conducted in several phases to establish the safety for use in human subjects.

One of the major enzyme families that play a role in how drugs are processed in the human body is the cytochrome P450 (CYP450) super-family. CYP450 enzymes are responsible for the oxidative metabolism of approximately $75 \%$ [34-36] of the marketed drugs in current clinical use. Understandably the study of CYP450 metabolic reactions is one of the important steps in current (pre-clinical) drug screening methods.

\subsubsection{Cytochrome P450}

CYP450 enzymes are so-called haemoproteins, which mean that they have a haem group tightly bound to the protein. An example of such a haem group within a CYP450 protein is shown in figure 2.6a. A haem group consists of an iron atom contained within a large heterocyclic organic ring called a porphyrin. The best known example of a haemoprotein is hemoglobin, which is responsible for the oxygen transport in red blood cells.

CYP450 enzymes catalyze the oxidation of all kinds of molecules. The basic, simplified catalytic cycle of CYP450 is shown in figure 2.6b [36-38]. In short, (beginning from the top, to the right) a target molecule (indicated by RH) binds to the active site of the CYP450 (1). Next, an electron is transferred from another enzyme (NADPH) resulting in a transfer of the haem iron atom into the ferrous (2+) state (2). In a next step, molecular oxygen binds to the haem iron atom (3). Another electron is transferred from NADPH, reducing the oxygen into a short-living, intermediate peroxo group (4). Next, the peroxo group is protonated twice, subsequently a water molecule is released (5). In the final step, the target molecule is oxidized and released after which the cycle can start again (6). The steps shown in figure 2.6b, do not necessarily follow in successive order around the circle. Also, several shortcuts (like the hydrogen peroxide shunt (S)) are known. 


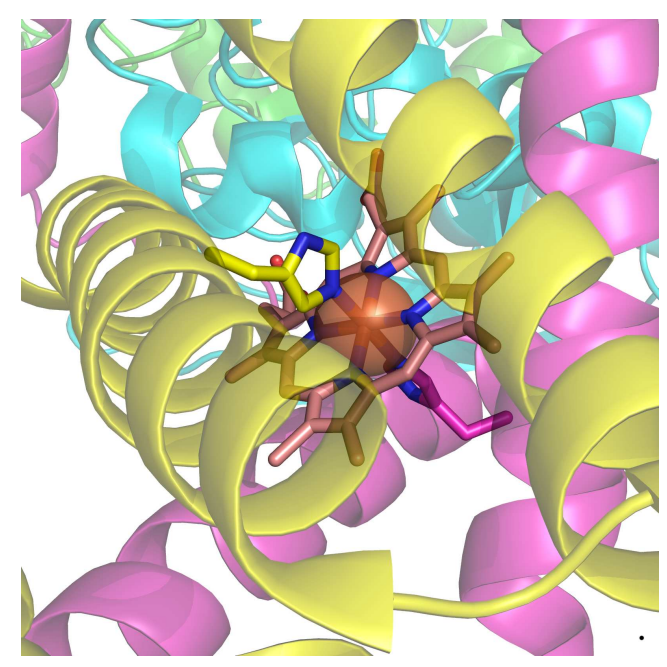

(a) [8]

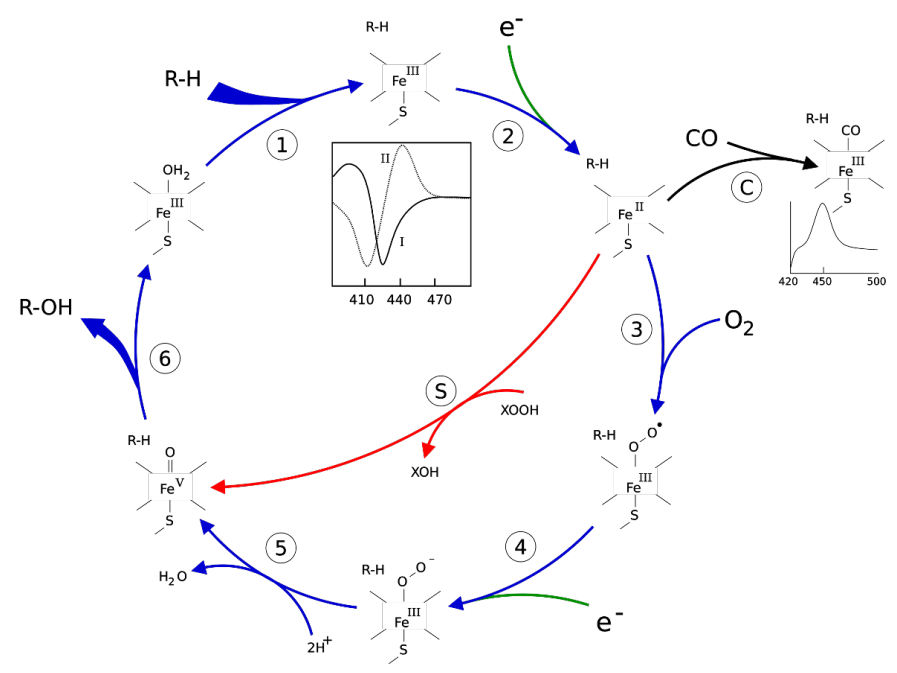

(b) [8]

Figure 2.6: (a) 3D picture of haem group within a CYP450 enzyme. (b) Basic catalytic cycle of CYP450. 
As stated before, CYP450 is a general name for a family of enzymes. Many different enzymes are known and structures have been studied in depth [39, 40]. CYP450 enzymes are not only found in humans but also in many other animals and plants [41]. In humans, CYP450 enzymes are mainly present in membranes of liver cells, but they are also found in e.g. the lung or the intestine [42]. CYP450 enzymes can be bought commercially, present within liver subcellular extracts prepared by differential ultracentrifugation. These extracts come in different flavors; microsomal ${ }^{4}$, cytosol or S9 extracts. Microsomal extracts contain mainly P450 enzymes, while cytosol extracts mainly contain enzymes which react with the metabolites formed after catalytic oxidation by CYP450. S9 extracts contain both enzymes and therefore gives a more comprehensive overview of the processes occurring in vivo.

In pharmacology, chemical reactions are often divided into two groups; phase1 and phase- 2 or conjugation reactions. Phase- 1 reactions usually precede phase-2 reactions, but a phase- 1 reaction is not always required for a phase-2 reaction to occur. Phase-1 reactions include oxidation, reduction or hydrolysis. Generally, phase-1 reactions generate more polar (water-soluble) and less active metabolites. However, phase-1 reactions can also convert an inactive drug into an active compound, which is referred to as bioactivation. Unfortunately, phase-1 reactions can also generate toxic metabolites. CYP450 is responsible for phase- 1 reactions only. Phase- 2 reactions usually involve covalent bonding of the drug or phase-1 metabolite to other (detoxifying) compounds like e.g. glutathione, a known antioxidant.

\subsubsection{Biomimetic modeling of CYP450}

In general, four in vitro methods have been recognized to mimic the in vivo CYP450 drug metabolism reactions [38]. The method most easy to transfer to the in vivo situation is the enzymatic model, which uses liver cell extracts. One of the major drawbacks of this method is that metabolic products might adhere to the cell membrane present in the liver cell extracts, making them unable to detect.

A second model is based on metalloporphyrin-containing systems [43]. The idea behind this second model is to mimic the reactive center of the haem group present in the CYP450 enzyme, using a similar molecular structure.

\footnotetext{
${ }^{4}$ vesicles derived from hepatocyte's endoplasmatic reticulum
} 
A third model is based on the EC-Fenton reaction [38]. This method can be regarded as a chemical way of oxidizing organic compounds using hydroxyl radicals. In the simplest approach, a Fenton reagent containing hydrogen peroxide and a ferrous salt is mixed with an organic compound to study the oxidation products. The simplified EC-Fenton is as follows:

$$
\mathrm{Fe}^{2+}+\mathrm{H}_{2} \mathrm{O}_{2} \longrightarrow \mathrm{Fe}^{3+}+\mathrm{OH}^{-}+\bullet \mathrm{OH}
$$

A more ingenious on-line EC-Fenton method is presented by Jurva et al. [44], where the $\mathrm{Fe}^{3+}$ is reduced in an on-line electrochemical flow-through cell such that the iron ions are recycled.

The three methods discussed so far are not within the scope of this thesis. In the next paragraph, the fourth method using direct electrochemical oxidation is discussed in detail. A good comparison of all four methods is discussed in several reviews and papers $[38,45,46]$.

Perhaps the most obvious method to induce oxidation reactions is to use direct electrochemical oxidation. In table 2.2, a comparison is shown of reactions known to occur in CYP450-based oxidation and direct electrochemical oxidation [45-47]. Most of the reactions observed by CYP450 catalysis are also observed using direct oxidation except for epoxidation ${ }^{5}$, alcohol and aldehyde oxidation reactions. The method is shown to be feasible in numerous studies [45, 47-68]. Examples include the study of metabolic products of clozapine (a antipsychotic drug) [55], amodiaquine (antimalarial agent), amsacrine and mitoxantrone (both intercalating antitumor agents) [57] and many more. Recently Nouri-Nigjeh et al. generated reactive oxygen species by electrochemical means to study their reaction with lidocaine to generate metabolic products via an alternative method [69]. Using this method both the $\mathrm{N}$-oxide and the N-dealkylation product of lidocaine are observed. The first is $\mathrm{H}_{2} \mathrm{O}_{2}$ mediated and the latter is the result of direct electrochemical oxidation. Direct EC oxidation might not always give sufficient yields of metabolic products. Another method like e.g. the EC-fenton or metalloporphyrin based systems might be more suited, as recommended in an overview published in a paper by Johansson et al. [45].

\footnotetext{
${ }^{5}$ Epoxidation is the formation of an epoxide group on the target molecule. An epoxide group is an $\mathrm{O}$-atom, bound to two $\mathrm{C}$-atoms. It is highly reactive and often used in adhesives such as epoxy glue.
} 
Table 2.2: Reactions known to be catalyzed by CYP450, compared to the reactions observed using direct oxidation in electrochemical cells.

\begin{tabular}{ll}
\hline $\begin{array}{l}\text { Phase-1 reactions catalyzed by } \\
\text { CYP450 [45, 46, 52] }\end{array}$ & $\begin{array}{l}\text { Reactions observed by direct ox- } \\
\text { idation }\end{array}$ \\
\hline \hline Hydroxylation & Benzylic and aromatic hydroxyla- \\
& tion [45] \\
& Allylic / aliphatic hydroxylation \\
& {$[47]$} \\
\hline N,O,S-Dealkylation & N,O-Dealkylation [45] \\
\hline Dehydrogenation & Dehydrogenation $[55,57]$ \\
\hline N,S,P-Oxidation & N,S,P-Oxidation $[45,52]$ \\
\hline Epoxidation & \\
\hline Alcohol and aldehyde oxidation & \\
\hline
\end{tabular}

Direct electrochemical oxidation is not the same as oxidation by CYP450 (as illustrated in table 2.2), but it can be used as complementary tool. Direct oxidation offers several advantages over liver cell microsomal incubation. It is a low-cost method, it can be done on-line in an automated fashion and is therefore a fast method. The feasibility of the direct oxidation method is shown in this thesis in chapter 3 and 4 . Liver cell microsomal incubations are still regarded as the golden standard, but in some cases direct electrochemical oxidation is shown to give more information (ch. 4) on the generated metabolites.

\subsubsection{Lab-on-Chip in CYP450-based drug screening}

Most of the research efforts in the field of lab on chip and CYP450 based screening are focused towards enzymatic oxidation of drugs. Benetton et al. [70] used a simple microreactor chip to let liver cell microsomes react with the target compound. Metabolic products were analyzed off-chip using ESIMS. More recent efforts were focused towards microscale culture of liver [71] or intestinal [72] cells in a chip. The major benefit of this approach is that cells survive for longer periods of time compared to cell culture of organ slices. Cell growth is influenced by microenvironmental stimuli, including neighboring cells, extracellular matrices, soluble factors and physical forces [71]. Lab on chip technology provides the tools to control the microenvi- 
ronment for successful cell culture, because dimensions of the cell culture chamber match the dimensions of cells $(10 \mu \mathrm{m})$ more closely.

\subsection{Electrochemistry}

Electrochemistry deals with the effects of electrical and chemical interrelations. This is mostly studied in chemical reactions where electrical charges or an electrical current play a role. Applications are for example batteries, fuel cells, electrochemical sensors and some types of displays or solar cells. Main topics in the field of electrochemistry are electrode reactions and redox reactions in solutions.

In light of the topic of this thesis, this section is limited to processes were chemical reactions take place at electrode surfaces. This section is therefore limited to faradaic processes only, meaning it will assume reactions were electrons are transferred between an electrode and redox active species in solution.

\subsubsection{Theory of faradaic processes}

This section is based on Bard and Faulkner [73] and chapter 9 of [74].

A typical electrochemical cell is illustrated in figure 2.7. This cell includes the following components: a container containing a solution (electrolyte), a working electrode, a reference electrode and a counter electrode. This cell is connected with electrical wiring to a potentiostat which is a box capable of applying voltages to the electrodes while measuring the current that flows through the cell.

A closer look at the working electrode in figure 2.7, reveals that an $F e^{2+}$ ion, can donate an electron to the electrode. This process can only occur if the energy of the electrons present inside the electrode is sufficiently low compared to the electron energy level in the $F e^{2+}$ ion. This can be achieved by applying a sufficiently positive potential to the working electrode, since the energy of the electrons is equal to the applied voltage multiplied with the (negative) elementary charge of the electron $\left(W_{e}=q \cdot V\right)$. The electron of the $\mathrm{Fe}^{2+}$ ion will favor the lowest energy state, which is in this case the electrode. The reaction in which an ion donates an electron to the electrode is 


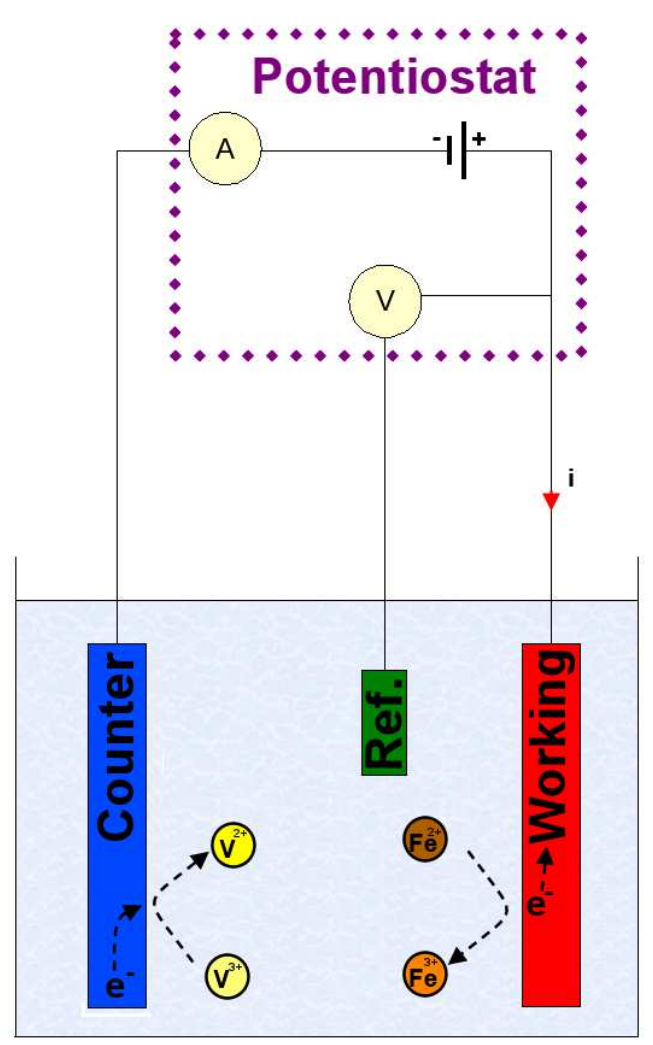

Figure 2.7: Typical three-electrode electrochemical cell used for studying faradaic processes. A and V indicate an current and potential meter respectively. 
called oxidation. Each ionic species (like e.g. $F e^{2+}$ ions) has is own electron energy level, which is an intrinsic property of that species.

In figure 2.7, another, opposite reaction is taking place at the counter electrode. In this example, a $V^{3+}$ ion receives an electron from the counter electrode, resulting in a $V^{2+}$ ion. The uptake of an electron by an ion from the electrode is called a reduction reaction. Again, this reaction can only take place if the energy of the electrons present in the electrode is sufficiently high compared to that of the $V^{2+}$ ion, such that the favorable position for the electrons is with the ion. Usually the processes occurring at the working electrode are of most interest to electrochemists. The counter electrode is added to the electrochemical cell to keep the electrical circuitry closed.

A voltage is always defined relative to another voltage. In electrical engineering, voltages are usually defined versus ground potential. In electrochemistry, this ground potential does not really apply. Therefore another reference potential is required to define the potential applied to the working (and in a lesser extend the counter) electrode. This reference potential is provided by the reference electrode. A good reference electrode provides a stable potential which is defined by a well-known and well defined electrochemical reaction occurring at the electrode-solution interface. An ideal reference electrode has a stable potential regardless of the current flowing through the electrode (nonpolarizable), the composition of the electrolyte, temperature and time [75]. It is difficult to combine all these properties into a single device, especially if the electrode needs to be small $\left(<1 \mathrm{~cm}^{3}\right)$ as well. In section 2.3.2.3, the issue of (miniaturized) reference electrodes is discussed in more depth.

\subsubsection{Reaction rate determining steps}

The rate at which the electrochemical reaction will take place depends on several factors. A generalized reaction is given in equation 2.2, in which an oxidized species $\mathrm{O}$ is reduced by an n-electron transfer into reduced species R.

$$
O+n \cdot e^{-} \underset{k_{b}}{\stackrel{k_{f}}{\rightleftharpoons}} R
$$

In figure 2.8, the pathway of a general electrode reaction is depicted. First, ions need to be transported to the electrode surface (mass transport). Next, a 
chemical reaction might precede or follow the actual transfer of electrons between ion and electrode. These chemical reactions can include protonation, dimerization or catalytic decomposition ${ }^{6}$ on the electrode surface. Another possible factor in the reaction rate might be adsorption, desorption or crystallization $^{7}$ at the electrode surface. The most obvious factor that can determine the reaction rate is the actual transfer of electrons between the electrode and the ion.

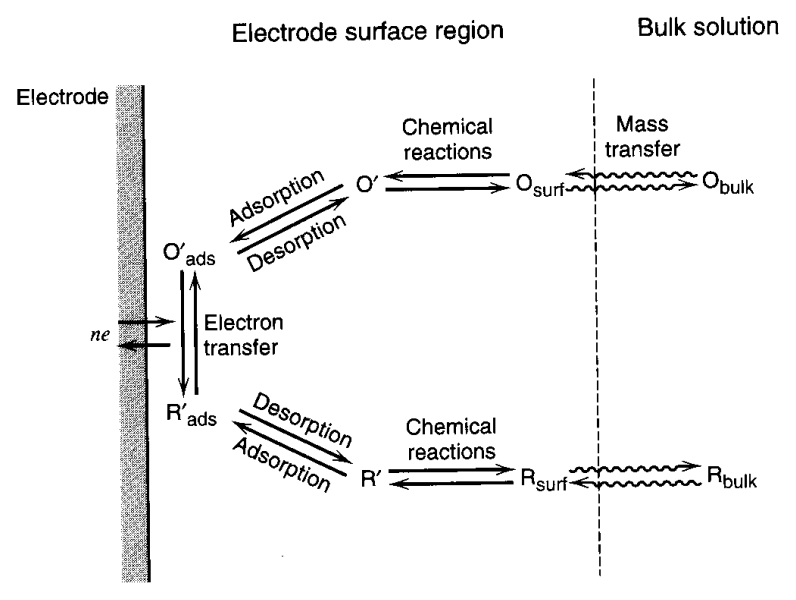

Figure 2.8: Rate determining steps for a typical electrochemical reaction at the electrode surface [73].

It is important to notice that not all steps play a role in every electrochemical reaction. In simple electrochemical processes, usually only mass transport and charge transfer are involved. In more complex reactions, multiple pathways might be possible or the reaction might be irreversible. In most cases one of the steps is the slowest, and therefore determining the overall reaction rate.

\footnotetext{
${ }^{6}$ The half-reaction that determines the potential of the normal hydrogen electrode is based on the catalytic decomposition of hydrogen gas into two hydrogen atoms, that are subsequently oxidized at the electrode surface. The platinum surface acts as catalyst [75].

${ }^{7}$ Crystallization also includes electrodepostion, e.g. in the halfreaction between a silver electrode and chloride ions, forming a silver chloride deposition layer on the electrode.
} 


\subsubsection{Butler-Volmer model}

Suppose the situation in figure 2.7 were no net current $\mathrm{i}$ is flowing towards the working electrode and only $\mathrm{Fe}^{2+}$ and $\mathrm{Fe}^{3+}$ ions are present in equal concentrations. In that case, the potential measured between the reference and working electrode is equal to the formal potential $\left(E^{0^{\prime}}\right)$ of the $F e^{2+} / F e^{3+}$ redox couple ${ }^{8}$ with respect to that reference electrode. If now the potential of the working electrode is slowly increased an oxidation reaction will start to occur. In 1905, J. Tafel already observed that the amount of current flowing is exponentially depending on the potential applied $\left(E_{\text {appl }}\right)$ with respect to the standard potential [76]. This observation is expressed with the Tafel equation

$$
\eta=E_{\text {appl }}-E^{0^{\prime}}=a+b \cdot \ln i / i_{0}
$$

in which $\eta$ is the overpotential and a and $\mathrm{b}$ are empirical constants, of which the latter is now known as the Tafel slope. A model describing electrode reaction kinetics matching to the Tafel equation (2.3), is given by the ButlerVolmer equations.

The net reaction rate $\left(v_{n e t}\right.$ in $\left.\mathrm{mol} \cdot \mathrm{m}^{-2} \cdot \mathrm{s}^{-1}\right)$ for the reaction given in equation 2.2 , can be subdivided in a forward and backward reaction rate by

$$
v_{n e t}=v_{f}-v_{b}=k_{f} \cdot C_{O}-k_{b} \cdot C_{R}
$$

in which $v_{f}$ and $v_{b}$ are the forward and backward reaction rates, $k_{f}$ and $k_{b}$ the forward and backward rate constants (in $\mathrm{m} / \mathrm{s}$ ) and $C_{O}$ and $C_{R}$ (in $\mathrm{mol}$. $m^{-3}$ ) the concentrations of oxidized and reduced species. For a one electron transfer reaction, the rate constants are defined by

$$
\begin{array}{r}
k_{f}=k_{s} \cdot e^{-\alpha \cdot F \cdot \eta /(R \cdot T)} \\
k_{b}=k_{s} \cdot e^{(1-\alpha) \cdot F \cdot \eta /(R \cdot T)}
\end{array}
$$

in which $k_{s}(\mathrm{~m} / \mathrm{s})$ is the standard rate constant and $\alpha$ the transfer coefficient (unitless), with its value between 0 and 1 , both for that particular reaction. F, $\mathrm{R}$ and $\mathrm{T}$ are the Faraday constant $(96485[\mathrm{C}]$ oulomb $/ \mathrm{mol})$, the gas constant

\footnotetext{
${ }^{8}$ Very often, the formal potential $\left(E^{0^{\prime}}\right)$ and the standard potential $\left(E^{0}\right)$ are confused in literature. The difference between the formal and the standard potential is that the formal potential includes the activity coefficients of the redox active species, while the standard potential does not. See 2.3.1.4 or section 2.1.6 in [73] for more details.
} 
$(8.31 \mathrm{~J} / K)$ and the temperature (in $K)$ respectively. The rate constant simply defines the rate at which the reaction will take place. In other words, it is a measure of how easy the redox couple is oxidized or reduced. The transfer coefficient is a measure of the symmetry of the reaction. For completely symmetrical reversible reactions, its value is 0.5 . For completely irreversible reactions its value is either 0 or 1 , depending on the direction in which the reaction occurs. For reactions with a two electron transfer, usually the transfer of one of the electrons define the overall reaction rate. Therefore $n$ is hardly ever included in equations 2.5 and 2.6 [73].

The measured current $\mathrm{i}(A)$ can be calculated from the net reaction rate by multiplying this rate with the surface area A of the electrode $\left(m^{2}\right)$, the number of electrons transferred in the reaction (n) and the Faraday constant (F).

$$
i=v_{n e t} \cdot n \cdot F \cdot A
$$

\subsubsection{Mass Transport}

In electrochemical systems, transport of ions can be initiated by three different mechanisms. If ions move due to a concentration gradient, the process is called diffusion. Ions with a positive or negative charge can also move due to an electrical force applied by an electric field, which is referred to as migration. With convection, the medium (electrolyte) itself is moving and dragging the ions along.

The three means of mass transport are described and combined in the NernstPlanck equation, resulting in a net flux $\left(N_{j}\right.$ in $\left.\mathrm{mol} \cdot \mathrm{m}^{-2} \cdot \mathrm{s}^{-1}\right)$ of ionic species $j$.

$$
\vec{N}_{j}=\underbrace{-D_{j} \nabla C_{j}}_{\text {diffusion }}-\underbrace{\frac{n_{j} F}{R T} D_{j} C_{j} \nabla \Phi}_{\text {migration }}+\underbrace{C_{j} \vec{u}}_{\text {convection }}
$$

In equation $2.8, D_{j}$ refers to the diffusion constant, $\Phi$ is the potential at each point in the electrolyte and $\vec{u}$ is a vector describing the fluid velocity of the electrolyte.

Often the Nernst-Planck equation is combined with the continuity equation, stating that a change in concentration in time must be equal to a change in flux in space, because of conservation of matter.

$$
\frac{\partial C_{j}}{\partial t}=-\nabla \cdot \vec{N}_{j}
$$




\subsubsection{Electrode potential}

Suppose once more the situation in figure 2.7 were no net current $i$ is flowing towards the working electrode and only $\mathrm{Fe}^{2+}$ (reduced, R) and $\mathrm{Fe}^{3+}$ (oxidized, $\mathrm{O}$ ) ions are present in equal concentrations. A certain formal potential $\left(E^{0^{\prime}}\right)$ is measured at the working electrode with respect to the reference electrode. This situation can be regarded as an equilibrium. If now the potential of the electrode is raised to $\mathrm{E}$, the concentrations of $\mathrm{R}$ and $\mathrm{O}$ are changed. To achieve this, a certain amount of work $\varepsilon$ (in $\mathrm{J} / \mathrm{mol}$ ) equal to

$$
\varepsilon=n\left(E-E^{0^{\prime}}\right) F
$$

needs to be added to the system to convert $1 \mathrm{~mol}$ of $\mathrm{R}$ into oxidized species O. Boltzmann statistics state that

$$
\frac{C_{O}}{C_{R}}=e^{-\varepsilon /(R \cdot T)}
$$

which can be regarded as the new ratio of ox/red ions due to 1 mole of oxidized ions gaining an extra energy of $\varepsilon \cdot 1$ joule. If equation 2.10 and 2.11 are combined and rewritten, we get the following result for the electrode potential

$$
E=E^{0^{\prime}}+\frac{R \cdot T}{n \cdot F} \ln \frac{C_{O}}{C_{R}}
$$

which is known as the Nernst equation ${ }^{9}$.

\subsubsection{Ohmic drop}

Ohmic drop is an undesired effect that hampers the proper determination of the working electrode potential with respect to the reference electrode. The theory of electrolyte conductivity and the basic working principle of a potentiostat need to be explained to understand this phenomenon.

In electrolytes current can flow by transporting charged ions, just as in electrical wires current flows by the movement of electrons. In both cases, conductivity can be regarded as a measure of how easy the charge carriers can

\footnotetext{
${ }^{9}$ The derivation of the Nernst equation is kept brief here. If derived more extensively via the concept of Gibbs free energy, the activity of redox active species should also be discussed. Based on the concept of activity it can be explained that if the oxidized or reduced species are present in solid or gaseous form the value of that species gets unity (1) concentration in the Nernst equation. See e.g. section 2.1.6 in [73] for more information.
} 
move through the medium under the influence of an electric field. In contrast to electrical wires, more than one type of charge carrier can contribute to the current in electrochemical systems. Therefore the equivalent conductivity $\kappa$ (in $\Omega^{-1} / m$ ) is defined in electrolytes as

$$
\kappa=F \sum_{j=1}^{m}\left|z_{j}\right| \cdot \mu_{j} \cdot C_{j}
$$

where $\mu_{j}$ (in $m^{2} \cdot V^{-1} \cdot s^{-1}$ ) is the mobility and $z_{j}$ the charge of ion $j$. The conductance $G$ (in $\Omega^{-1}$ ) of a fluidic channel with cross-sectional area $A$ and length $l$ can be calculated by

$$
G=\kappa \frac{A}{l}
$$

In figure 2.9, the basic working principle of a potentiostat is depicted. The circle represents the electrochemical cell with a three electrode configuration with the working (WE), counter (CE) and reference electrode (RE). A control loop is provided by the (operational) amplifier $(\mathrm{K})$. The current through the counter and working electrode is determined via the voltage drop over measurement resistor $R_{m}$ using ohms law. Ideally, the input resistance of the negative input terminal is approaching an infinitely high value, such that almost no current is flowing through the reference electrode. The desired working electrode potential is provided by an adjustable voltage source $E_{d}$.

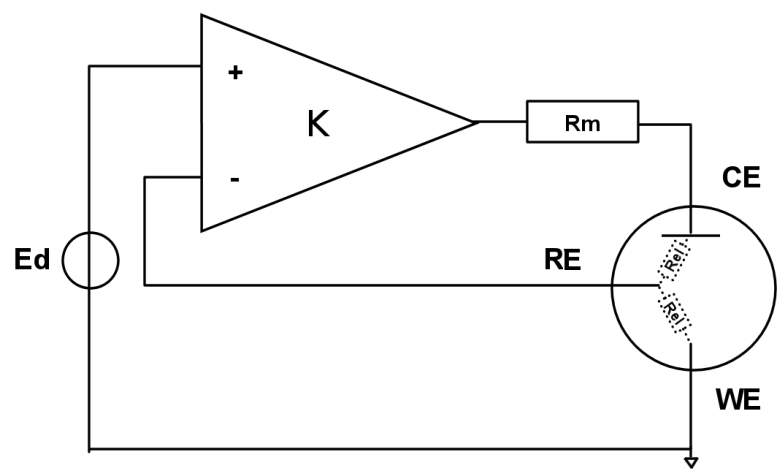

Figure 2.9: Basic working principle of a potentiostat.

The general transfer function of an operational amplifier, describing the potential $U$ at the output, by the potentials at the inputs is

$$
U=K\left(u^{+}-u^{-}\right)
$$


where $\mathrm{K}$ is the amplification factor which is usually very large $\left(>10^{5}\right)$. If now equation 2.15 is applied to the schematic shown in figure 2.9 with $E_{r m}$ the potential drop over measurement resistor $R_{m}$, the result is

$$
E_{C}-E_{r m}=K\left(E_{d}+E_{W}-E_{R}\right)
$$

If this resulting equation is rewritten and $\mathrm{K}$ is assumed to approach infinity, the following result is obtained:

$$
E_{W}=E_{R}-E_{d}
$$

which is the desired situation; the potential of the working electrode $\left(E_{W}\right)$ can precisely controlled by $E_{d}$ with respect to $E_{R}$.

Suppose an extra resistance $R_{e l}$ is present inside the electrolyte between the counter and working electrode, while a non-constant current is flowing. A potential (ohmic drop) $E_{o d}=i \cdot R_{e l}$ will drop over this electrolyte resistance as a function of the current, causing the potential measured at the reference electrode to change into $E_{R^{\prime}}=E_{R}+E_{\text {od }}$. The new resulting working electrode potential now becomes

$$
E_{W}=\left(E_{R}+E_{o d}\right)-E_{d}
$$

Equation 2.18 indicates that the working electrode potential will also change since the working electrode potential is now no longer simply the sum of the constant reference electrode potential $E_{R}$ and the desired potential $E_{d}$. As the schematic and equations presented here describe a simplified model, it is not easy to compensate for this effect after the measurement because the ohmic drop changes in time, e.g. by capacitive effects.

In general it is a good practice to place the reference electrode as close as possible to the working electrode and to add supporting (non-reacting) ions to the solution to minimize the resistance between working and reference electrode.

\subsubsection{Measurement techniques}

Modern potentiostats can be controlled using a wide variety of measurement protocols or methods. Each method has its own specific advantages which make them suited for a specific measurement task. In light of the rest of this thesis, only chronoamperometry (CA), cyclic voltammetry (CV) and square wave voltammetry (SWV) are discussed here. 


\section{Chronoamperometry}

In chronoamperometry, a fixed potential is applied to the working electrode while the current is measured as a function of time. In general, depletion of ions will occur at the surface of the electrode resulting in a slow decay of the measured current over time. The measured response will obey the Cottrell equation:

$$
i(t)=n \cdot F \cdot A \cdot C^{*} \sqrt{\frac{D}{\pi \cdot t}}
$$

with $C^{*}$ the concentration in the bulk of the electrolyte ${ }^{10}$. The measured current will be different for cases where the electrode size is smaller. The Cottrell equation is also not valid if the development of the diffusion profile is hindered (e.g. due to the presence of other geometric structures like neighboring electrodes) or convective mass transport is present.

\section{Cyclic voltammetry}

Cyclic voltammetry (CV) is a controlled potential technique, just like chronoamperometry. The main difference is that in $\mathrm{CV}$ the potential is varied as depicted in figure $2.10 \mathrm{a}$. The current is measured as a function of potential, resulting in a graph similar to what is shown in figure $2.10 \mathrm{~b}$ for a fast reacting, reversible redox couple. The lines in both figures are color-coded for explanation purposes.

At the start of the scan (red, section A) overall reaction rates, and thus the measured current, are determined by the speed of the charge transfer from electrode to solution, as described by the Butler-Volmer equations. At a certain moment the overpotential is sufficiently large and charge transfer is no longer the rate limiting step. Instead, depletion of ions close to the electrode surface will occur and reaction rates are now limited by mass transport, as indicated in blue (section B). The scan direction is now reversed and at a certain point a reduction reaction will start to take place (green, section C), noticeable by the negative currents measured. Following the same reasoning,

\footnotetext{
${ }^{10}$ The Cottrell equation can be derived from the Nernst-Planck and continuity equation for a one-dimensional problem assuming diffusion as the only means of mass transport. The equation is valid for planar electrodes with dimensions above $\sim 25 \mu \mathrm{m}$ (see [73], page 161176).
} 
the rate of the reduction reaction will be limited by the charge transfer first, followed by depletion of oxidized ions at the electrode surface (pink, section D). Finally the scan is reversed again towards positive potentials, after which the process is repeated (cyan, section E).

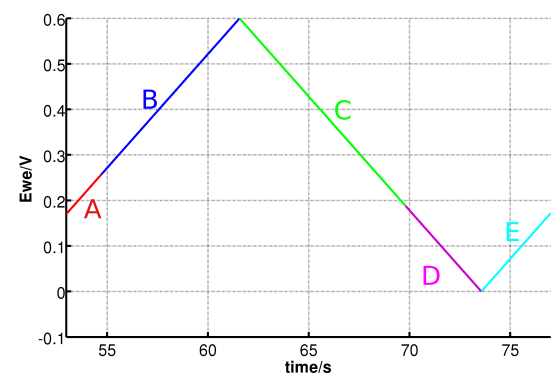

(a) Potential vs. time.

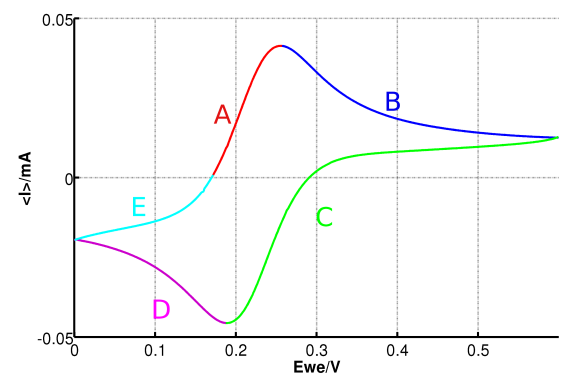

(b) Current vs. potential.

Figure 2.10: (a) Potentials applied in a typical CV measurement. (b) Typical cyclic voltammogram. $E^{0^{\prime}}$ is $0.23 \mathrm{~V}$ for this specific redox couple.

For a trained electrochemist, a CV diagram contains a wealth of information. The position of the peaks indicate the standard potential of a reversible redox couple, since the charge transfer limited parts of the curve are determined by the overpotential which is a function of applied minus standard potential. Also, the height of the peaks, amongst other factors, relate to the concentration of the redox active species. The separation between the negative and positive peak also holds information as it should always be close to $59 \mathrm{mV}$ for a fast reacting, completely reversible one-electron transfer reaction. Interpreting $\mathrm{CV}$ diagrams is often a challenging task because many factors can change the shape of the curve, especially if multiple or non-reversible redox active species are involved. Therefore, the reader is referred to a more complete discussion of CV analysis in [73], chapter 6.

\section{Square wave voltammetry}

In square wave voltammetry (SWV) a square wave is applied to the working electrode as depicted in figure 2.11a. After each period, the square wave is raised with an offset $\Delta E_{s}$. Instead of plotting the current directly versus potential, a difference in current $i_{f}-i_{r}$ is determined from the current measured 
from each half-cycle of the square wave. A typical result that can be obtained using SWV is shown in figure $2.11 \mathrm{~b}$.

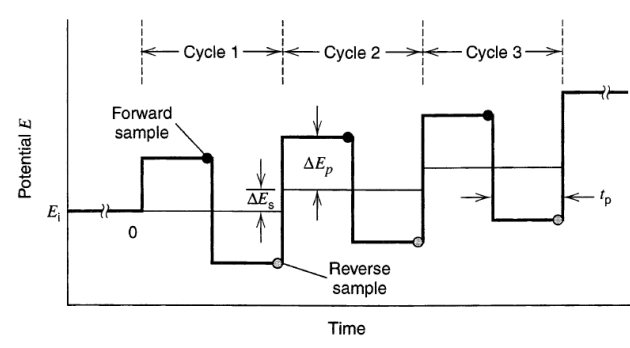

(a) From [73]

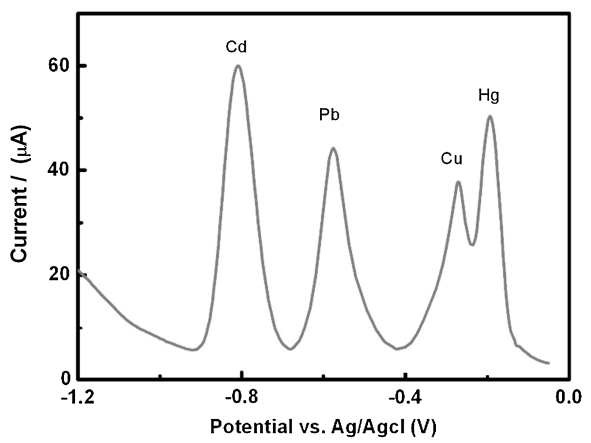

(b) From [77]

Figure 2.11: (a) Applied potential in a typical SWV measurement. (b) Typical result of a $S W V$ measurement.

SWV has the advantage that the result is easy to interpret in terms of concentrations and standard potentials of the redox active species present in solution. The peak is located on the $\mathrm{x}$-axis at the standard potential of the corresponding redox couple. The peak height can be related to the concentration by the following equation [73]

$$
i_{p}=n \cdot F \cdot A \cdot C_{j}^{*} \cdot \psi_{p} \sqrt{\frac{D_{j}}{\pi t_{p}}}
$$

with $t_{p}$ the width of one plateau (half of the square wave period) and $\psi_{p}$ a factor determined by the peak height $\Delta E_{p}$ and $\Delta E_{s} . \psi_{p}$ can be found in table 7.3.2 in [73]. SWV is a special case of differential pulse voltammetry (DPV), because the duty cycle of the square wave is equal to 0.5 . A similar expression for the peak current is known for DPV (see p.290 of [73]), but is not treated here. In chapter 6 , a new measurement technique is proposed which shows a familiar result to the measurements obtained with SWV. This new technique adds the benefit of more selectivity for reversible redox couples using the redox cycling effect.

\subsubsection{Redox Cycling}

Redox cycling (RC) is an effect that can occur if two electrodes are placed in close proximity, such that the concentration profiles caused by faradaic 
conversions overlap. One of the electrode structures frequently used for RC is the interdigitated array (IDA) electrode (see fig. 2.12a). The IDA electrode is composed of two working electrodes formed by two sets of fingers, which are entangled to get as much concentration profile overlap as possible.

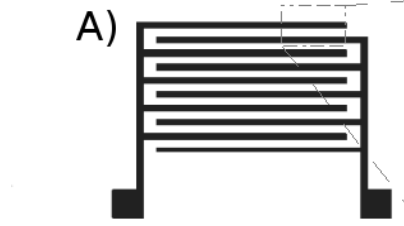

B)

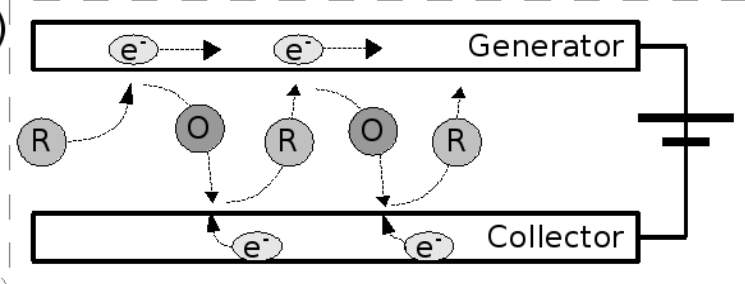

Figure 2.12: (a) Top view of an interdigitated array electrode. (b) Redox Cycling explained by a cycling ion between a generator and collector electrode.

In case of the general reversible redox couple, as described by eq. 2.2, a single reduced molecule $\mathrm{R}$ can be oxidized at the generator electrode. The resulting oxidized species $\mathrm{O}$ will diffuse away from the generator electrode and might reach the collector electrode, where it can be reduced again into the starting product R. Again by diffusion the product $\mathrm{R}$ can reach the generator electrode a second time, where it will be oxidized again. This cycle is called the $\mathrm{RC}$ effect. It is important to notice that a single reversible redox active molecule contributes one electron to the measured current for each cycle. The redox cyling effect can therefore amplify the measured current in a chemical manner. Moreover it can contribute to an enhanced selectivity, since only the current of reversible redox couples is amplified by the RC effect. RC can only occur if one electrode is at a reducing potential and one electrode is at an oxidizing potential with respect to a certain redox couple with standard potential $E^{0^{\prime}}$. Therefore, RC can provide even more selectivity by choosing appropriate potentials around $E^{0^{\prime}}$ for a specific redox couple.

Biologically relevant compounds that show reversible behavior are e.g. dopamine, catechol and some of the quinone or quinone imine products from oxidative metabolic conversion by cytochrome P450. The latter two compounds are often highly reactive, electrophilic compounds formed upon oxidative metabolism [78]. Also, one of the oxidation products in tyrosine-specific electrochemical protein cleavage is a quinone product. This makes the RC effect also interesting as detection tool in drug screening applications. More- 
over it can be considered as monitoring tool for the oxidation and electrochemical cleavage efficiency of proteins.

\subsubsection{Design of miniaturized electrochemical cells}

So far, two applications for electrochemical conversions are discussed. One application lays in direct oxidation of drugs for mimicry of the cytochrome P450 metabolism. The other application discusses the possible use of tyrosine specific electrochemical cleavage of proteins as tool in the field of proteomics. Both applications focus on high turnover electrochemical conversions to gain sufficient oxidation products for further analysis.

Lab on chip technology provides the advantage of high surface to volume ratios by using small microfluidic channels. Since mass transport by diffusion is a slow process in general, lab on chip technology can help to get a decent conversion efficiency by minimizing the time required for ions to diffuse towards the electrode surface.

In the following subsections the designs of electrochemical cells for electrochemical conversions are discussed. First, some of the fabrication platforms known for fabricating microfluidic chips are briefly discussed. Next, several electrode materials are treated for use as either working or reference electrode. Finally, several existing macroscale and microscale cell designs known from literature are reviewed.

\subsubsection{Fabrication platforms}

Several platforms can be distinguished for fabricating microfluidic chips, based on the substrate material used. Probably one of the best known materials is poly(dimethylsiloxane) (PDMS), which has gained a lot of attention from the microfluidic community over the past two decades [79]. The main reason for the popularity of PDMS is its ease of use and the possibility for rapid prototyping. It is a flexible, low-cost, transparent polymer, which is usually poured onto a microfabricated mold of silicon, glass or photoresist SU-8. After curing, it can be pealed off the mold and bonded to another PDMS or glass layer. Using PDMS it is easy to fabricate valves, pumps and multilayer devices, opening the possibility to fabricate complex integrated microfluidic systems $[80,81]$. One of the major drawbacks of PDMS, especially in light of the previously discussed applications, is the adsorption of organic solvents 
(causing swelling) and small molecules [82]. Another issue is that PDMS chips, once bonded, can only withstand moderate pressure (approx. below $1 \mathrm{MPa})$.

Other, more traditional, materials that are used frequently to fabricate microfluidic chips are glass and silicon. Glass and silicon are more difficult to process, but offer the advantage of more inertness to various (aggressive) chemicals. Also the microfabrication can be conducted with more precision. For example, it is possible to fabricate shallow channels in glass or silicon with nanometer precision and high aspect ratios without the risk of a collapse. Usually the fabrication of these channels involve reactive ion etching in silicon, or wet etching using HF in glass or silicon [83]. A glass wafer can be bonded directly onto another glass substrate, using an annealing step at around $600^{\circ} \mathrm{C}$. Anodic bonding between glass-silicon is also possible and provides a very strong and reliable bond. Chips fabricated using the direct or anodic bonding method can withstand pressures up to at least $30 \mathrm{MPa}$, which is important for e.g. HPLC applications [84].

More (polymer) substrate materials are known, like poly(methylmethacrylate) (PMMA), polycarbonate (PC), polyester, polystyrene (PS) and the negative photoresist SU-8. However, most of these polymers show limited resistance against organic or acidic solvents [79, 82, 85]. One of the more promising materials is the Cyclic olefin (co)polymers (COCs or COPs). This material is sold commercially by a number of manufacturers under the brand names of Apel, Arton, Topas, Zeonex and Zeonor. COP offers many of the advantages of glass/silicon based chips, like the resistance to (strong) acids, bases, alcohols and esters [86]. It also shows excellent optical properties like good optical transparency in the near UV range and low autofluorescence. Moreover, it has the added benefit of ease in fabrication. Fabrication methods include laser ablation, micromilling, injection moulding, hot embossing and nanoimprint lithography [86-88]. The first two methods are more suited for rapid prototyping, while the latter three are more suited as replication methods. Microfluidic chips composed of COP are shown to be robust, since channel burst pressures of up to $34.6 \mathrm{MPa}$ have been reported [89].

The major benefits and drawbacks of the various fabrication materials are summarized in table 2.3 for convenience. Which material is best for the design of an electrochemical cell depends on the application. For HPLC or 
Table 2.3: Major benefits and drawbacks of various fabrication materials for microfluidic chips.

\begin{tabular}{lllll}
\hline Material & $\begin{array}{l}\text { Easy fab- } \\
\text { rication }\end{array}$ & Costs & $\begin{array}{l}\text { Chem. re- } \\
\text { sistance }\end{array}$ & $\begin{array}{l}\text { Max. } \\
\text { pressure }\end{array}$ \\
\hline \hline Glass / silicon & - & - & ++ & $30 \mathrm{MPa}$ \\
\hline PDMS & ++ & + & - & $<1 \mathrm{MPa}$ \\
\hline $\mathrm{COC}$ & + & + & + & $34.6 \mathrm{MPa}$ \\
\hline $\begin{array}{l}\text { PMMA/PC/PS/ } \\
\text { polyester }\end{array}$ & + & + & - & $\begin{array}{l}\text { Varies per } \\
\text { mat. }\end{array}$ \\
\hline
\end{tabular}

mass spectrometric applications glass/silicon or COC based materials seem most appropriate since high pressures or (partly) organic solvents can be expected. Silicon needs to be passivated first with e.g. an oxide layer for its conducting properties, before it can be combined with electrodes.

\subsubsection{Work electrode materials}

A good working electrode, in general, shows a fast and reproducible charge transfer with the targeted reaction of interest. Ideally, the measured current for this reaction is not disturbed by background reactions that take place at the same potential of the targeted reaction. E.g. a reaction that will take place at a potential of $1.5 \mathrm{~V}^{11}$, is not easy to measure with a platinum electrode without the interference of water molecules being oxidized to form oxygen and protons. Other factors that can influence the choice of working electrode material are cost, toxicity and robustness.

Likely the most used materials in aqueous media are carbon, gold and platinum, of which the latter is probably the most favorable for its electrochemical inertness and ease of fabrication [90]. The main drawback of platinum however is the $\operatorname{cost}^{12}$ and the limited potential window at negative potentials, especially in acidic conditions (see [73], fig. E.2).

${ }^{11} 1.5 \mathrm{~V}$ defined versus a normal hydrogen reference electrode (NHE). The potential of the NHE is arbitrarily set to $0 \mathrm{~V}$ and acts as reference point to all other redox reaction potentials. See table C.1. of ref. [73]

${ }^{12}$ In 2010 platinum was priced 1600 US dollar per troy oz, which is around $33 \%$ more expensive as gold. 
Gold shows similar behavior to platinum, but adds the added drawback that its surface can oxidize easily at positive potentials ( 1.12V vs. NHE [91]). However, this also makes gold a useful material to modify the surface to enhance or catalyze specific reactions by forming monolayers at the gold surface.

Carbon electrodes can be used in a very wide potential window $(-1.3 \mathrm{~V}$ to $+1 \mathrm{~V}$ [73]). Carbon electrodes are used mostly in the form of (porous) glassy carbon or carbon paste. The latter form is fragile and therefore not suited for flow-through cells.

One of the materials that has gained a lot of interest of the electrochemical community over the last decade is doped diamond. The most striking feature of doped diamond is its high overpotential for oxygen and hydrogen evolution, resulting in the widest potential window in aqueous media reported so far (approx. 3.5V) [92]. This large potential window opens the possibility for a very effective hydroxyl $(O H \cdot)$ radical production in aqueous media.

One important aspect in light of the previously described applications is compatibility with cleanroom fabrication processes. Metal electrodes are easy to fabricate using sputtering techniques to form thin film electrodes [83]. Carbon electrodes can be made using either pyrolysis of organic materials like photoresist or deposition of graphite-like films using various sputtering techniques [93].

Fabrication of doped diamond electrodes is more difficult compared to metal or carbon electrode fabrication. Fabrication techniques include chemical vapor deposition (CVD), vacuum high temperature annealing (at $1550^{\circ} \mathrm{C}$ ) and immobilization of doped diamond particles onto a (conducting) substrate. A mixture of several gases is required for CVD of doped diamond. Usually hydrogen gas is used as carrier, while methane or a mixture of acetone/methane is used as carbon source. Furthermore, a dopant gas needs to be added to the mixture to get sufficient conductivity, which can be e.g. nitrogen [94], boron containing gases [95, 96] or phosphorus containing gases [97]. Doped diamond electrodes can be shaped into e.g. microelectrodes by selective deposition [98] or etching of thin films [96].

In light of the previously discussed applications, thin metal films are the most easily fabricated materials in a cleanroom environment. Gold is not suited for 
its adsorptive properties. Carbon electrodes are not compatible with the standard cleaning procedures used during the bonding of glass or silicon based substrates. Doped diamond is definitely a promising material, but the requirement of the various gases makes the fabrication process difficult and not routinely available on CVD systems. Since the applications require an oxidative potential, the small potential window of platinum towards reducing potentials is not a big issue. Platinum seems therefore the most likely candidate as working electrode material.

\subsubsection{Macroscale reference electrodes}

In general, an ideal reference electrode (RE) has the following properties:

- It is non-polarizable ( $E_{R}$ not a function of $i$ through the reference electrode).

- If it does show slight polarizable behavior, it should at least be reversible ( $E-i$ behavior always the same, no hysteresis or memory effects).

- Its potential is not influenced by the composition of the electrolyte $\left(E_{R}\right.$ not a function of various concentrations of ionic species).

- It is temperature independent $\left(E_{R}\right.$ not a function of $\left.T\right)$.

- Its potential is time independent; no drift ( $E_{R}$ not a function of $t$ ).

Some more (soft) demands can be added to the list, such as low-costs, easy maintenance and ease of use. For a miniaturized EC cell, two more demands are important that add limitations on the widely used large scale REs. A miniaturized RE is logically small (volume of electrode in same range or smaller than the volume of the microfluidic channels) and should be easy to fabricate.

Three types of well-known macroscale REs are depicted in figure 2.13. The normal hydrogen electrode (NHE) is constructed of a platinum wire, put in a solution with $\mathrm{pH} 0$, while hydrogen gas is bubbled over the platinum electrode keeping the partial pressure of the hydrogen gas at $1 \mathrm{~atm}$. Not depicted in the figure is a salt-bridge which is usually present to connected the NHE with the 


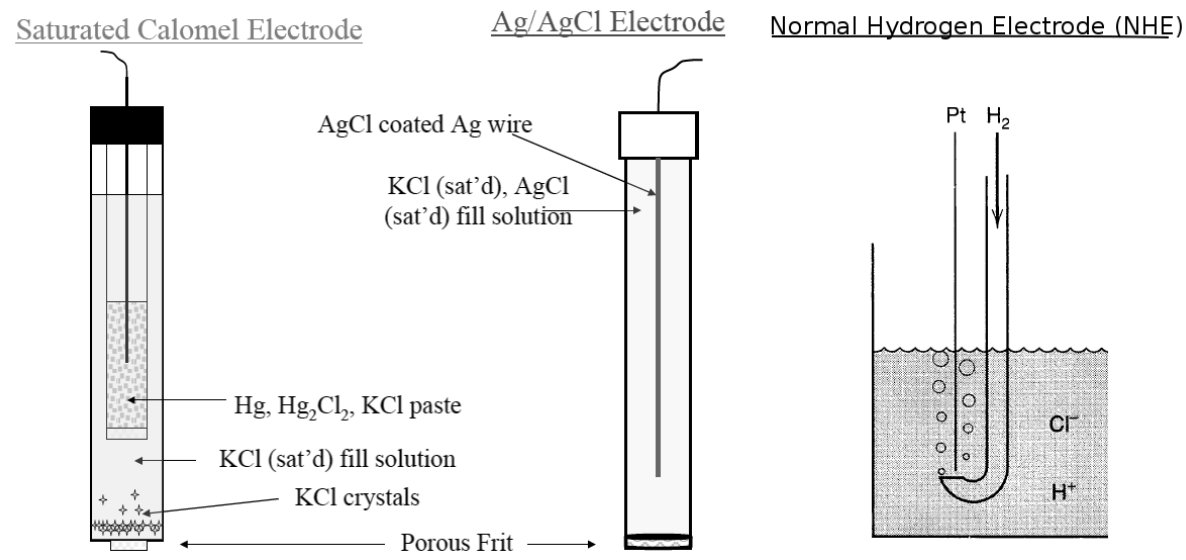

Figure 2.13: Three types of well-known reference electrodes. (1) saturated calomel electrode (2) Silver/Silverchloride electrode (3) Normal hydrogen electrode. [73, 99].

rest of the electrochemical setup, while maintaining a constant $\mathrm{pH}$ around the platinum wire. The potential of the NHE is given by the following reaction:

$$
2 H_{a q}^{+}+2 e^{-} \leftrightarrow H_{2(g)}
$$

This reaction is actually a two-step process; first dissolved hydrogen gas in the solution is split into two hydrogen atoms at the electrode surface.

$$
H_{2(a q)} \leftrightarrow 2 H
$$

This first step requires a metal like e.g. platinum to catalyze this reaction. [75]. Secondly, the hydrogen atoms are oxidized into protons, as described below.

$$
2 H \leftrightarrow 2 H_{a q}^{+}+2 e^{-}
$$

The potential of the NHE is defined by the Nernst equation (eq.2.12), where $E^{0^{\prime}}$ is 0 by definition and the fraction inside the logarithm is 1 due to the $1 \mathrm{~atm}$ partial pressure of the hydrogen gas and the $\mathrm{pH}$ of 0 of the solution. Since the second term in the Nernst equation is zero, the NHE shows no temperature dependence.

The saturated calomel electrode (SCE) consists of a platinum wire inside a mixture of mercurous chloride $\left(\mathrm{Hg}_{2} \mathrm{Cl}_{2}\right.$, calomel) and mecury metal. This 
mixture is kept in an inner compartment, which is in contact via a porous membrane or glass frit with a surrounding compartment that is filled with a saturated $\mathrm{KCl}$ solution to keep the chloride ion concentration constant. Contact with the external electrolyte is provided by a second porous membrane or glass frit. The potential of the SCE is based on the reaction between calomel and mercury:

$$
\mathrm{Hg}_{2} \mathrm{Cl}_{2(s)}+2 e^{-} \rightarrow 2 \mathrm{Hg}_{(l)}+2 \mathrm{Cl}_{\text {aq,sat'd }}^{-}
$$

Again using the Nernst equation, it follows that the potential of the SCE is fixed since the activities of the calomel and mercury are constant, and the concentration of chloride is also constant due to the saturated inner electrolyte.

The construction of the silver/silverchloride $(\mathrm{Ag} / \mathrm{AgCl})$ electrode is somewhat similar to the SCE in the sense that it also is formed around an inner compartment filled with a saturated $\mathrm{KCl}$ solution. The electrode however, is made of silver covered with a solid silverchloride deposition layer. The reaction that defines its potential is given by:

$$
A g C l_{(s)}+e^{-} \rightarrow A g_{(s)}+C l_{a q, s a t^{\prime} d}^{-}
$$

The potential is defined in a similar way to the SCE.

Both the SCE and the $\mathrm{Ag} / \mathrm{AgCl}$ electrode have some temperature dependence. Moreover it is important to know that the liquid junction potential over the frit or membrane is negligible, since the mobilities of the chloride $\left(7.9 \cdot 10^{-8} \mathrm{~m}^{2} / \mathrm{Vs}\right)$ and potassium $\left(7.6 \cdot 10^{-8} \mathrm{~m}^{2} / \mathrm{Vs}\right)$ ions are almost equal at room temperature. Compared to the NHE the SCE and $\mathrm{Ag} / \mathrm{AgCl}$ electrodes are more practical to use, since it is usually difficult to keep the right conditions ( $\mathrm{pH} 0$, 1atm hydrogen gas pressure) for the NHE.

\subsubsection{Miniaturized reference electrodes}

So far, miniaturized reference electrodes (MREs) have not been able to reach a similar performance compared to the macroscale REs [100]. Efforts towards miniaturization can be categorized into a few different directions, and are often based on the previously discussed macroscale REs. The first category is based on miniaturization of the entire macroscale equivalent RE. That includes the fabrication of a small cavity which contains the inner electrolyte 
[101-106] for the $\mathrm{Ag} / \mathrm{AgCl}$ or SCE [107] RE or miniaturization of the entire NHE [108].

The second category is also based on the $\mathrm{Ag} / \mathrm{AgCl} \mathrm{RE}$, and can be distinguished by elimination of the inner electrolyte. In this approach the $\mathrm{Ag} / \mathrm{AgCl}$ electrode is shielded to keep the chloride concentration constant by use of a polymer coating, a NAFION membrane or a hydrogel [109-111]. In all categories discussed so far, the long term stability is the main issue.

The third category is based on the so-called pseudo-reference electrodes. The term pseudo refers to the fact that these electrodes are not real REs, since their potential is based on the composition of the electrolyte. The best example of a pseudo-reference is an $\mathrm{Ag} / \mathrm{AgCl}$ wire simply put directly into the electrolyte. The $\mathrm{Ag} / \mathrm{AgCl}$ pseudo- $\mathrm{RE}$ is therefore sensitive to the chloride concentration in the electrolyte (see e.g. [112] and many others). Silver can be evaporated or sputtered onto a substrate. Silverchloride can also be evaporated or made on top of the silver by electrochemical means. In the next chapters ( 3 and 4) palladium and iridium oxide are used as pseudo RE. Therefore these two electrode materials are discussed in more depth.

\section{Palladium}

Palladium (Pd) can provide a stable potential similar to the mechanism of the NHE. It has similar catalytic properties as platinum, required for the reaction given by 2.22 to occur. Unlike platinum, Pd can buffer protons by including them into the crystal lattice [113]. The buffering properties can assist to keep a constant proton concentration at the electrode surface, and therefore a constant potential, provided that the ratio of protons to palladium does not reach more than 0.6. Above this value, a metalhydride is formed and the buffered protons can no longer participate in the reaction [75]. For these buffering properties, Pd is considered as material to fabricate (pseudo-)REs [113, 114]. Like the platinum wire of the NHE, palladium is $\mathrm{pH}$ sensitive. Its potential is therefore $\mathrm{pH}$ dependent.

Pd thin-films can be formed by sputtering. The Pd thin-film is not so robust as a platinum thin-film. Firstly, it cannot withstand temperatures above $500^{\circ} \mathrm{C}[115]$. Secondly, we have noticed that the uptake of protons creates mechanical stress in the thin-film which can lead to detachment from the 
substrate. Therefore Pd thin-films are not compatible with some of the more aggressive (acidic) cleaning procedures used in the cleanroom.

\section{Iridium oxide}

Iridium oxide ( $\mathrm{IrOx}$ ) is a very exciting material for its unique (electro)chemical, electrochromic and other physical properties. IrOx is used for applications in a wide range of fields, including $\mathrm{pH}$-sensing [116], electrochromic devices and optical information storage, chlorine or oxygen evolution, neural stimulation [117], field emission cathodes, and advanced memory technology. More recently it is gaining renewed interest as electrode material for supercapacitors [118].

IrOx film fabrication processes include anodic growth, thermal growth and sputtering. The anodic iridium oxide film (AIROF) is produced by cycling iridium metal in acid at potentials between $-0.25 \mathrm{~V}$ and $1.25 \mathrm{~V}$ vs. SCE [116]. Sputtered iridium oxide film (SIROF) is fabricated by sputtering an iridium target while introducing oxygen gas into the vacuum chamber [119]. Less common techniques include the growth of IrOx by sol-gel dip-coating from $\mathrm{IrCl}_{4}$ solution [120], the pulsed laser deposition from Ir targets in reactive atmosphere [121], and the deposition of $\mathrm{IrO}_{2}$ nano-rods by metal oxide chemical vapor deposition $[122,123]$. Each way of fabricating IrOx gives unique electrochemical properties [124]. If only sputtered iridium oxide films (SIROFs) are considered, already the sputter parameters like substrate temperature, oxygen pressure and vacuum pressure are of great influence in the electrochemical behavior $[119,125,126]$. CV diagrams measured with SIROFs exhibit a typical 'featureless' or capacitor-like shape [118, 119, 127], compared to AIROFs [117, 128, 129].

The $\mathrm{pH}$ sensitivity of IrOx makes it a possible candidate to act as pseudoRE, as already demonstrated in literature [130-133]. The redox reactions responsible for the $\mathrm{pH}$ sensitivity of $\mathrm{IrOx}$ are still under debate. According to Olthuis [128], the following reaction might explain the supra-nernstian behavior of AIROFs:

$$
2 \mathrm{Ir}(\mathrm{OH})_{2} \mathrm{O}^{-}+\mathrm{H}_{2} \mathrm{O} \longleftrightarrow \mathrm{Ir}_{2} \mathrm{O}(\mathrm{OH})_{3} \mathrm{O}_{3}^{3-}+3 \mathrm{H}^{+}+2 e^{-}
$$

Supra-nernstian behavior is referring to a $\mathrm{pH}$ sensitivity larger than $59 \mathrm{mV} / \mathrm{pH}$, which is explained by the reaction given and the Nernst equation $2.3 \cdot(-3 / 2)$. 
$(R T / F)=-88.5 \mathrm{mV} / \mathrm{pH}$. According to Midgley [116], the potential is determined by the reaction:

$$
\begin{array}{r}
\operatorname{IrO}_{x / 2}\left(\mathrm{H}_{2} \mathrm{O}\right)_{y}+n \cdot \mathrm{OH}^{-}+(m-n / 2) \mathrm{H}_{2} \mathrm{O} \\
\longleftrightarrow \operatorname{IrO}_{(x+n) / 2}\left(\mathrm{H}_{2} \mathrm{O}\right)_{y+m}+n \cdot e^{-}
\end{array}
$$

i.e., it involves a change in the average oxidation state $\mathrm{x}$ of iridium in the oxide phase, and is affected by the extent of hydration. According to Midgley, these electrodes suffer some interference from sodium and oxygen, but none from hydrogen, $\mathrm{Fe}^{2+}, \mathrm{Fe}^{3+}, \mathrm{Cu}^{2+}$ and $\mathrm{Ag}^{+}$.

If size is the most important factor, the MRE concepts from the first category (complete miniaturization of macroscale equivalents) do not qualify. Compared to pseudo-MREs (3rd category), the properties provided by the coated or covered MREs (2nd category) show better potential stability for limited time periods. In disposable, or potential critical applications the covered MREs seem the best choice. However, this increased potential stability comes at a price of a more complex fabrication process and the requirement of regeneration if the MRE is used multiple times. Therefore the pseudo-MRE, e.g. composed of $\mathrm{Ag} / \mathrm{AgCl}, \mathrm{Pd}$ or $\mathrm{IrOx}$ thin films, seems the best candidate in terms of ease of fabrication and ease of use. However, special care has to be taken into using the pseudo-MRE in appropriate electrolytes with known concentrations of the potential determining ions.

\subsubsection{Existing integrated cell designs}

Before giving an overview of existing cell designs, it must be clarified what is regarded as an electrochemical cell in this section. An electrochemical cell contains at least one, and preferably two or more electrodes placed in a fixed and well-defined volume of electrolyte. The electrolyte compartment and one or more electrodes must be integrated into a single device to be included in this section. Moreover, this section is constrained to cells used in faradaic processes only. Several electrochemical cell designs that fit this definition have been published. These publications can be divided into two distinct categories based on their size.

In this chapter, the miniaturized cell category refers to at least one dimension of the cell volume being in the micrometer range or smaller. Moreover, the 
volume of the total cell, including electrodes and substrate material, must be below a few cubic centimeters. The tip of a scanning electrochemical microscope (SECM) placed in close proximity to a conducting surface can also be regarded as an electrochemical cell. However, the total setup of the SECM tip and positioner do not qualify for the limiting condition of fitting into a few cubic centimeters. Also, the tip and the electrolyte compartment are two distinct parts in the SECM setup. Although very interesting results have been published in this field (e.g. single molecule detection [134]), SECM is not discussed in this section.

The other category is based on large scale cell designs. An electrochemical cell designed to obtain significant conversion efficiency (high turn-over rate) will look different to a cell designed towards detection, especially in terms of electrode area. The large-scale category is therefore mainly focused towards cells designed for electrochemical conversions.

\subsubsection{Large scale cell designs}

In a review by Baumann and Karst, an overview is given of the electrochemical cell designs used for the generation of oxidative metabolism products [46]. Moreover, Prudent and Girault reviewed modifications to electrospray ionization interfaces with the purpose to study electrochemical conversions [23]. The designs presented in these two reviews include flow-through (FT) cells with porous working electrodes [50, 135], thin-layer (TL) cells were the analyte is flushed in a thin-layer over the working electrode [136-138] or cells were the electrospray ionization interface (ESI) is used as electrochemical converter [139, 140].

A.

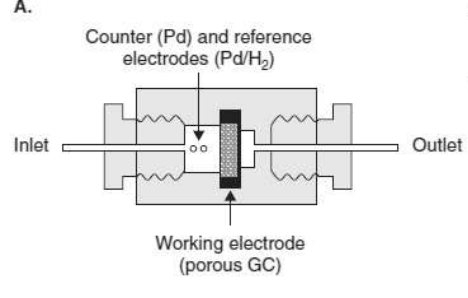

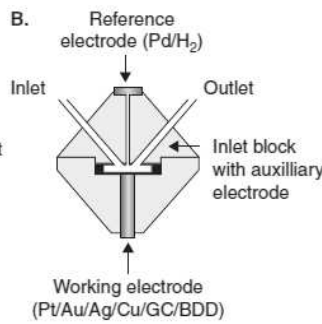

c.

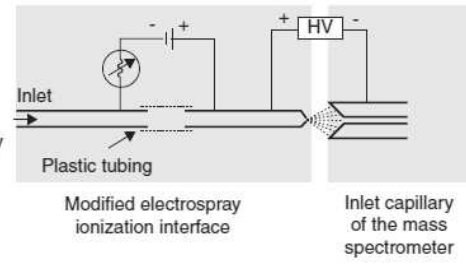

Figure 2.14: Overview of different kinds of electrochemical cell designs, as reviewed by Baumann and Karst [46]. a) flow-through design based on a porous electrode, b) thin-layer design, c) tip of ESI interface used as part of electrochemical cell. 
A schematic overview of the different cell designs is shown in figure 2.14. In figure $2.14 \mathrm{a}$, the flow-through design is depicted. In a flow-through cell, analyte is flushed through the (porous glassy carbon) electrode. In the case of the commercially available coulochem cells from ESA, the reference and counter electrode are made of palladium. The major benefit of this cell is the high turnover-rates due to a large surface to volume ratio of the working electrode. The major disadvantage is the risk for clogging of the porous electrode and the use of the pd pseudo-reference electrode.

The thin-layer design is shown in figure $2.14 \mathrm{~b}$. A thin layer of fluid is transported between two plastic body parts separated by a thin gasket, the latter defines the thickness of the fluid layer. Two channels drilled into the upper body part provide a fluidic inlet and outlet. The working electrode material is replaceable by dismounting it from the lower body part. The reference electrode is also replaceable via a larger hole in the top body part. This cell design can be bought commercially from Antec Leyden (AL). Working electrode materials include platinum, gold, silver, copper, glassy carbon and boron doped diamond, while the reference electrode can be of the $\mathrm{Ag} / \mathrm{AgCl}$ (with and without salt bridge) or (pseudo-ref.) Pd or Pt type. The major advantage of the thin layer design is its versatility, but the design shows lower turn-over rates at higher flow rates compared to the flow-through cells.

The basic working principle for ESI based electrochemical cells is shown in figure $2.14 \mathrm{c}$. In the basic setup, a potential for electrochemical conversions is applied between two conducting tubes, connected with a piece of nonconducting tube. The tube downstream is usually part of the ESI interface, and therefore connected to the high voltage power supply of the MS. A more advanced version of this cell is shown by $\mathrm{Xu}$ et al. [139], which include a $\mathrm{Ag} / \mathrm{Ag}+$ in $0.01 \mathrm{M} \mathrm{CH}_{3} \mathrm{CN}$ reference and a platinum wire as working electrode. Fused silica (FS) tubing acts as spacer between the working and counter electrode. This counter electrode is made of a larger tube fitted around the FS tubing, and acts simultaneously as tip for the ESI interface. The benefit of using the ESI tip as electrochemical converter is that it opens up the possibility to study shorter living compounds. However, in general the turn-over rates are low. Moreover, extreme care needs to be taken to avoid the hazard of electric shock from the high voltage power supply of the ESI interface. Floating (and electrically well insulated) potentiostats might be required if the ESI tip is not connected to ground potential. 
A detailed review of these different cells would only reproduce the already existing reviews $[23,46]$, but a summary of work conducted with these cells is included in table 2.4.

\subsubsection{Miniaturized cell designs}

\section{Planar designs}

The easiest way in terms of fabrication for practicing EC in small volumes, is to fabricate planar electrodes onto a substrate. A good example of such an approach is presented by Morita and coworkers [148]. Three planar, square electrodes are fabricated using standard sputtering, lift-off and lithographic cleanroom techniques. They use gold as working and counter electrode material. One of the three gold electrodes is converted to a silver pseudo-reference by covering it with a small drop of silver epoxy. Simply photoresist is used as insulator for the connecting wires, but this is not always resistant to the chemicals from the electrolyte. Their device was used to study ultra micro electrode (UME) behavior.

Many other papers present planar electrodes [149, 150], including individually addressable arrays of microelectrodes [151]. Suzuki and co-workers presented a three-electrode cell with a well-performing reference electrode based on a silver/silverchloride electrode, placed in a small cavity filled with $\mathrm{KCl}$ solution [152]. A schematic overview of their device is shown in figure 2.15a. On one side of a glass substrate, platinum working and counter electrodes are sputtered, while on the other side an $\mathrm{Ag} / \mathrm{AgCl}$ electrode is deposited. A cavity is etched in another silicon substrate, also providing holes for filling and a small capillary that acts as liquid junction to prevent the $\mathrm{KCl}$ to diffuse out of the cavity.

\section{Flow-through designs}

If the planar design is covered with a substrate containing microfluidic channels, inlets and outlets, a microfluidic electrochemical flow-through cell is obtained. This approach is demonstrated using various substrates like glass/PDMS [157, 158], PET [159] and Polystyrol [160]. This fabrication method requires two substrates, one for the fluidic channels and one for the electrodes. Alignment during bonding of these two substrates can be difficult, especially if small electrodes are considered. 
Table 2.4: Overview of known electrochemical cell designs (GH=graphite, GC=glassy carbon, see text for other abbreviations).

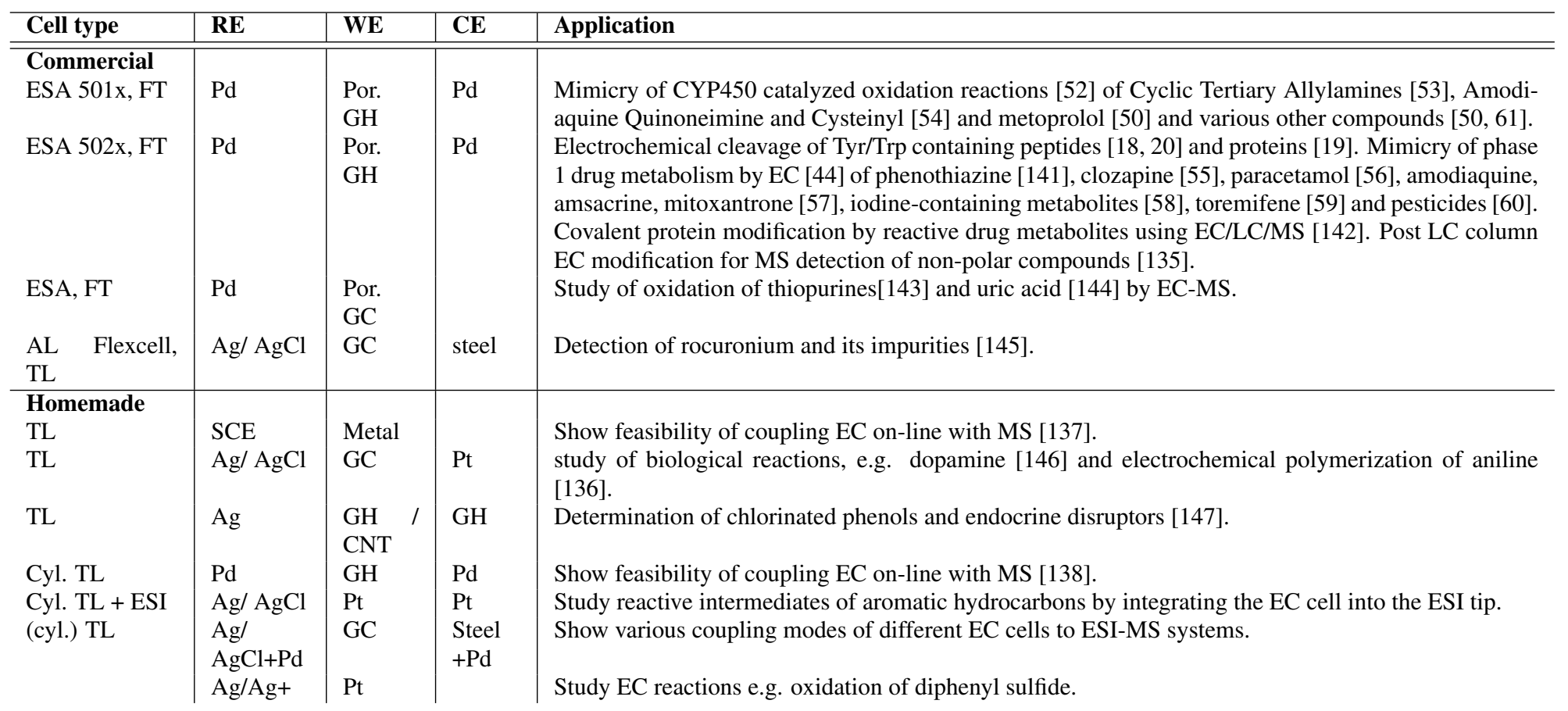




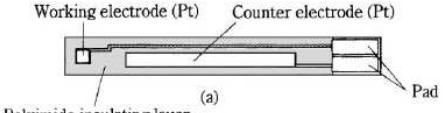

Polyimide insulating layer

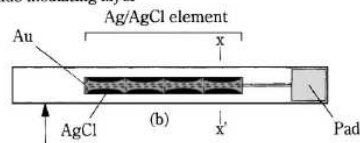

Bonded surfaces Container in which the electrolyte

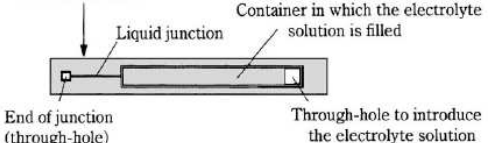
(through-hole)

(d)

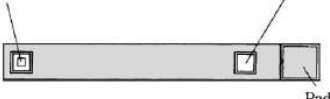

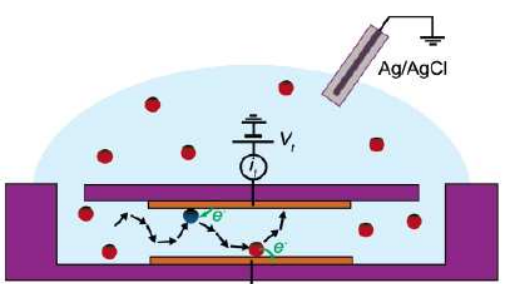
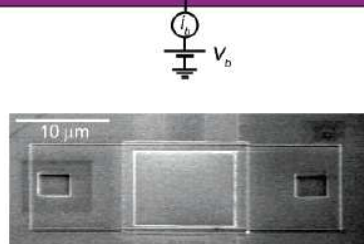

(a) Planar electrode design with (b) Two planar electrodes separated $\mathrm{Ag} / \mathrm{AgCl}$ sat. $\mathrm{KCl}$ reference elec- by a nanofluidic channel for redox trode compartment [152]. cycling measurements [153].
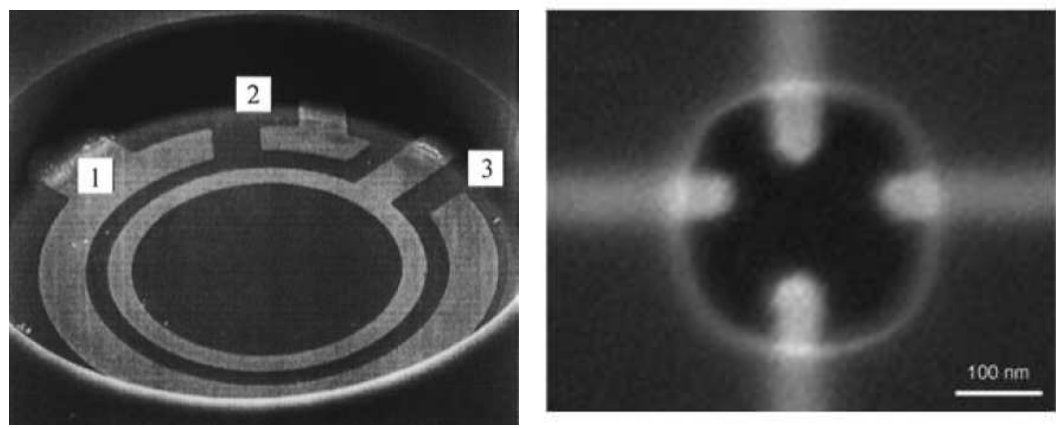

(c) Microwell with 3 electrodes at (d) Microwell with attoliter volume and the bottom for cell monitoring [154]. 4 nanometer sized electrodes [155].
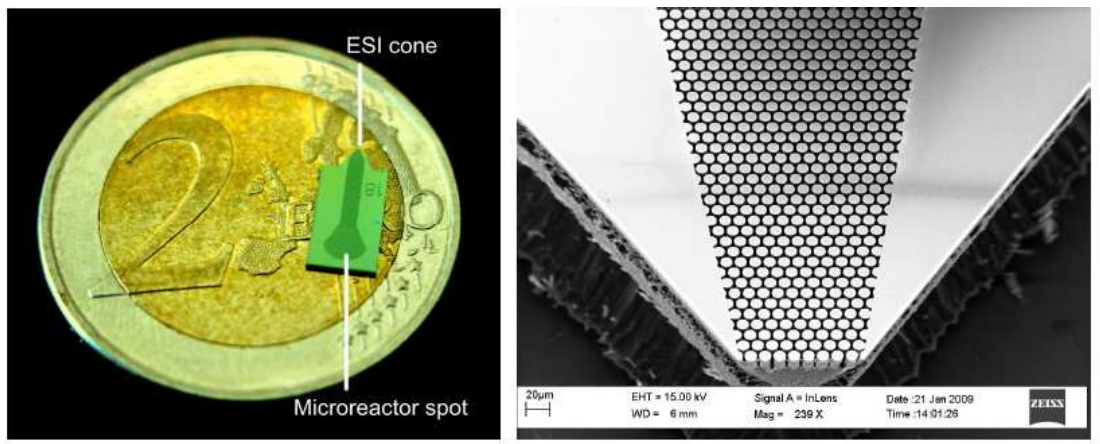

(e) Photocatalytic oxidation and ESI device for drug metabolism studies [156].

Figure 2.15: Various miniaturized electrochemical cell designs (see text, for details). 
Several other methods have been published for fabricating flow-through cells. Probably one of the easier methods is to use a piece of plexiglass, with drilled microfluidic channels and inserted wires as electrodes [161, 162]. The drawback of this approach is that the size of the microfluidic channels is limited by the size of the drill. Therefore the internal volumes of those cells is generally quite large (several microliter to milliliters).

Another easy fabrication method comprises the use of fluorinated ethylene propylene (FEP, shrink tubing), which is shrunk and simultaneously melted around a channel template (a tungsten wire) [163]. Electrodes are melted into the FEP either perpendicular, or parallel to the tungsten channel template. Afterwards, the tungsten wire is removed to open the channel. The major drawback of this method is that it is not easy to predict the area of the electrode that is in contact with the electrolyte.

A more controlled, easy fabrication method is presented by Rossier et al. [164]. A $50 \mu \mathrm{m}$ thick polyimide sheet covered with $5 \mu \mathrm{m}$ of copper on both sides is processed in a two step process. Each step is composed of photolithography, copper etching and plasma etching (of polyimide) to form electrodes and microfluidic channels. Finally the copper is coated with gold for more compatibility with the electrochemical processes and the microchannel is closed by a PET cover.

Liljegren et al. fabricated a flow-through cell specifically designed for conversions in EC-MS applications [165]. Their cell is based on thin gold wires, wound around a $50 \mu \mathrm{m}$ diam. stainless steel wire channel template casted in PDMS. An ESI needle is also integrated into the total chip. Using this cell, subsecond transfer times between the EC cell and the MS signal are reported. However, the major drawback of this cell is the limited conversion efficiency of $30 \%$ at a flow rate of $0.5 \mu \mathrm{L} / \mathrm{min}$ for the oxidation products of dopamine. Moreover, PDMS is not really suitable for MS applications, since PDMS tends to adsorb ions and swell if exposed to organic solvents like the frequently used acetonitrile [82].

\section{Well-based designs}

Several papers have been published reporting electrodes placed in microwells $[166,167]$. One of the purposes of such a system is to study redox active 
species excreted by cells or bacteria $[154,168,169]$. The system of Bratten and coworkers is a good example of such a system, as it incorporates a threeelectrode system with a silver/silverchloride pseudo reference electrode, and gold working and counter electrodes placed at the bottom of the well. The rather thick insulating polyimide layer for the connecting wires also defines the shape of the well, as depicted in figure 2.15c.

Another purpose of a well based system is shown by Baldwin and coworkers [170]. They have placed a two-electrode EC cell at the outlet of a electrophoresis channel. The well is formed by the fluidic through-hole in the top substrate. The cell is used as detector of redox active species after electrophoretic separation of e.g. dopamine.

Significant research effort is put in fabricating nanometer sized electrodes. Lemay et al. reports the fabrication of a 15 by $15 \mathrm{~nm}$ square gold electrode inside a pyramid-shaped 310nm deep well etched in silicon [171]. The fabrication method uses silicon on insulator (SOI) wafers, etched using $\mathrm{KOH}$ to control the size of the well. However, additional electrodes are still required to use these nanometer sized electrodes for electrochemical measurements. A very small well containing a four-electrode configuration is presented by Li and coworkers [155]. In their devices, nanometer sized gold and platinum electrodes are fabricated using e-beam lithography. A photoresist layer acts as insulating layer for the connecting wires and also defines the size of the well. The final result is depicted in figure $2.15 \mathrm{~d}$. The smallest device obtained using this fabrication method has electrodes with a width of $60 \mathrm{~nm}$. The well volume is approximately 18 attoliter $\left(10^{-18} \mathrm{~L}\right)$. Interestingly, at such small spacing counter, reference and working electrode largely interfere because diffusion profiles overlap almost immediately, producing cyclic voltammograms with irregular shapes.

\section{Other designs}

A miniaturized device for electrochemical conversions worth mentioning is the device shown by Nissila and coworkers [156, 172], although their device does not fully qualify for the limits defined at the beginning of this section. The main operating principle of their device is based on the photocatalytic properties of the $\mathrm{TiO}_{2}$ pillars present on their device, as depicted in figure 2.15e. under influence of UV light, an oxidizing potential becomes available 
to oxidize products present in the electrolyte between the pillars. The device also has an integrated ESI needle. Electrical forces pull on the liquid cone at the ESI tip, generating a spray. The ESI needle therefore drives the liquid through the pillars into the MS. This device is used to mimic the oxidative metabolism of verapamil, a known calcium channel blocker used as antiarrhythmic drug.

The last example is shown in 2.15b, fabricated by Zevenbergen et al.[153]. This device is fabricated using sacrificial layer etching of silicondioxide to produce two planar, parallel platinum electrodes of 12 by 12 and 10 by $10 \mu \mathrm{m}$, spaced at $282 \mathrm{~nm}$ from each other by a rectangular nanofluidic channel. This device is used to demonstrate high redox cycling amplification of up to 400 times. Moreover, the authors demonstrate (near single molecule) detection of approximately 70 molecules.

An overview of all literature discussed in this section is presented in table 2.5.

\subsection{Summary and outlook}

In this chapter, two possible applications for on-chip electrochemistry have been introduced. An overview of the role of Lab-on-Chip technology in these two areas is given. In the field of drug screening, so far only large cells have been used to mimic the oxidative metabolism of the cytochrome P450 enzyme. A miniaturized cell can offer specific advantages, since the surface to volume ratio in a lab-on-chip is greatly enhanced. Thereby, the time required for ions to diffuse towards an electrode surface can be greatly reduced. A miniaturized cell might show a better conversion efficiency using various electrode materials. Moreover, less analyte is required since cell volume and dead volumes can also be reduced.

Electrochemical cleavage of proteins offers specific advantages in the speed of analysis and the possibility to couple the electrochemical cell directly online to a liquid chromatography (LC) - MS system. The entire setup is thus fully instrumental and allows full automation. Two challenges that remain for this alternative protein digestion method is to increase the rather low cleavage yields together with decreasing adsorption issues at the electrode surface. Using the same argument of increased surface to volume ratio in lab-on-chip systems, the use of a miniaturized electrochemical cell might be helpful to 
Table 2.5: Overview of miniaturized electrochemical cell designs ( $*=e$ stimated from paper).

\begin{tabular}{|c|c|c|c|c|c|}
\hline Who & Cell description & Electrode mat. & Substrate & Typical dim. & Purpose \\
\hline \multicolumn{6}{|l|}{ Planar } \\
\hline Suzuki [152] & $\begin{array}{l}\text { Planar } \mathrm{WE}+\mathrm{CE} \text { with integrated liq. } \\
\text { junction } \mathrm{Ag} / \mathrm{AgCl} \text { ref. el. }\end{array}$ & $\begin{array}{l}\mathrm{Pt} \quad \mathrm{WE}+\mathrm{CE}, \\
\mathrm{Ag} / \mathrm{AgCl} \mathrm{RE}\end{array}$ & Glass + SI & $\begin{array}{l}0.25 \mathrm{~mm}^{2} \text { WE, } \\
3.2 \mathrm{~mm}^{2} \mathrm{CE}\end{array}$ & $\begin{array}{l}\text { Demonstrate } 3 \text {-el cell with stable } \\
\text { miniaturized ref. }\end{array}$ \\
\hline Morita [148] & Two planar square sized electrodes & Au WE, Ag RE & $\mathrm{Si} / \mathrm{SiO}_{2}$ & $120-2800 \mu m^{2}$ & $\begin{array}{l}\text { Study microelectrode behavior of } \\
\text { small electrodes. }\end{array}$ \\
\hline Lakard [149] & Two planar square sized electrodes & $\begin{array}{l}\mathrm{Pt}(+\mathrm{Ag} \text { or Poly- } \\
\text { mer })\end{array}$ & $\mathrm{Si} / \mathrm{SiO}_{2}$ & $1 \mu m^{2}$ & $\begin{array}{l}\text { Study e.g. deposition of Polyani- } \\
\text { line }\end{array}$ \\
\hline Sandison [150] & Microdisk array & Gold & $\begin{array}{l}\mathrm{Si} / \mathrm{SiO}_{2}+ \\
\text { PMMA }\end{array}$ & $\begin{array}{l}\text { 220-1100nm } \\
\text { diam. }\end{array}$ & $\begin{array}{l}\text { Show feasibility of nano-imprint- } \\
\text { lithography for microdisk fab. }\end{array}$ \\
\hline Zoski [151] & $\begin{array}{l}\text { Individually addressable array of } \\
\text { microdisk electrodes. }\end{array}$ & $\mathrm{Pt}$ & $\mathrm{Si} / \mathrm{SiO}_{2}$ & $10 \mu m$ diam. & $\begin{array}{l}\text { Show fabrication of individually } \\
\text { addressable microdisk array }\end{array}$ \\
\hline \multicolumn{6}{|l|}{ Flow-through } \\
\hline Suzuki [157] & $\begin{array}{l}\text { Integrated } \mu \text { Fluidic system with EC } \\
\text { actuated valves and pumps }\end{array}$ & $\begin{array}{l}\mathrm{Pt} \text { black WE, } \\
\mathrm{Ag} / \mathrm{AgCl} \mathrm{RE}+\mathrm{CE}\end{array}$ & $\begin{array}{l}\text { Glass } \\
\text { PDMS }\end{array}$ & $\begin{array}{l}0.04-6.7 \mathrm{~mm}^{2} \\
160 \mathrm{~mL}^{*}\end{array}$ & $\begin{array}{l}\text { Show feasibility of electrolysis as } \\
\text { pumping and valving mechanism }\end{array}$ \\
\hline Liljegren [165] & $\begin{array}{l}\text { Cylindrical cell with microcoil elec- } \\
\text { trodes for EC conversions in MS- } \\
\text { appl. }\end{array}$ & $\mathrm{Au}$ & $\begin{array}{l}\text { PMMA }+ \\
\text { PDMS }\end{array}$ & $\begin{array}{l}0.05-0.10 \mathrm{~mm}^{2} * \\
\text { unknown vol. }\end{array}$ & $\begin{array}{l}\text { Study EC conversions by EC-MS } \\
\text { of e.g. dopamine }\end{array}$ \\
\hline Bohm [162] & $\begin{array}{l}\text { Cell with } \mathrm{WE} \text { and } \mathrm{RE} \text {, in contact } \\
\text { with analyte and } \mathrm{CE} \text { via dialysis } \\
\text { tubing }\end{array}$ & $\begin{array}{l}\text { Pt WE, } \mathrm{Ag} / \mathrm{AgCl} \\
\text { RE, Pt CE }\end{array}$ & Plexiglass & $0.5 \mathrm{~mm}^{2} * ; 4 \mu L$ & $\begin{array}{l}\text { Detection of e.g. lactate using en- } \\
\text { zymes in WE cell cavity. }\end{array}$ \\
\hline Hranicek [161] & $\begin{array}{l}\text { Various plexiglass flow through } \\
\text { cells }\end{array}$ & $\mathrm{Pb}$ WE, Pt CE & Plexiglass & $\begin{array}{l}29-126 \mathrm{~mm}^{2} \\
214-340 \mathrm{~mm}^{3}\end{array}$ & $\begin{array}{l}\text { Hydride generation in atomic ab- } \\
\text { sorption spectrometry }\end{array}$ \\
\hline Kim [158] & $\begin{array}{l}\text { PDMS based flow through cell with } \\
3 \text { el + salt bridge }\end{array}$ & $\mathrm{Pt}+\mathrm{Ag} / \mathrm{AgCl}$ & $\begin{array}{l}\text { Glass } \\
\text { PDMS }\end{array}$ & $\begin{array}{l}3.1 \mathrm{~mm}^{2} \mathrm{WE}, 12.6 \\
\mathrm{~mm}^{2} \mathrm{CE} ; 35 \mathrm{~mL}\end{array}$ & $\begin{array}{l}\text { Show miniaturized RE based on } \\
\text { pDADMAC hydrogel salt bridge }\end{array}$ \\
\hline Sahlin [163] & $\begin{array}{l}\text { Polymer, cylindrical flow through } \\
\text { cell with } 2 \text { active EC spots }\end{array}$ & Carbon or Pt & $\begin{array}{l}\text { F.E. propy- } \\
\text { lene (FEP) }\end{array}$ & $\begin{array}{l}\text { Diam ch. } 13- \\
25 \mu m .\end{array}$ & $\begin{array}{l}\text { Easy fabrication method for min. } \\
\text { flow cells. }\end{array}$ \\
\hline Rossier [159] & $\begin{array}{l}\text { Planar or facing two-electrode cell } \\
\text { inside a microfluidic channel }\end{array}$ & Carbon & PET & $\begin{array}{l}450 \mu m^{2}-2 \mathrm{E} 3 \\
\mu m^{2} ; 48-120 \mathrm{pL}\end{array}$ & $\begin{array}{l}\text { Study EC processes in small vol- } \\
\text { umes }\end{array}$ \\
\hline Rossier [164] & $\begin{array}{l}\text { (4) Microdisk electrodes in a mi- } \\
\text { crochannel }\end{array}$ & $\mathrm{Cu}$ coated with $\mathrm{Au}$ & $\begin{array}{l}\text { Polyimide }+ \\
\text { PET }\end{array}$ & $\begin{array}{l}52-120 \mu m \\
\text { diam.; } 60 \mathrm{~nL}\end{array}$ & $\begin{array}{l}\text { Show novel fab. method based on } \\
\text { PI / PET substrates. }\end{array}$ \\
\hline Ueno [160] & $\begin{array}{l}\text { Three electrode system in a mi- } \\
\text { crofluidic channel }\end{array}$ & $\begin{array}{l}\mathrm{Au} \text { WE+CE, Ag } \\
\mathrm{RE}\end{array}$ & Polystyrol & $4700 \mu m^{2} ; 40 \mathrm{~nL}$ & $\begin{array}{l}\text { Study RC in confined spaces un- } \\
\text { der flow conditions }\end{array}$ \\
\hline
\end{tabular}




\begin{tabular}{|c|c|c|c|c|c|}
\hline Who & Cell description & Electrode mat. & Substrate & Typical dim. & Purpose \\
\hline Well-based & & & & & \\
\hline $\mathrm{Li}[155]$ & $\begin{array}{l}\text { Femto- to attoliter nanowell cell } \\
\text { on a chip with } 3 \text { or } 4 \text { el. }\end{array}$ & $\mathrm{Au}$ or $\mathrm{Pt}$ & $\mathrm{Si} / \mathrm{SiO}_{2}$ & $\begin{array}{l}9 \mu m^{2}-\sim 4 \mathrm{E} 3 n m^{2} * ; 140 \mathrm{fL} \\
-\sim 18 \mathrm{aL}\end{array}$ & $\begin{array}{l}\text { Fundamental insight in EC pro- } \\
\text { cesses in nano/attoliter cells. }\end{array}$ \\
\hline $\begin{array}{l}\text { Bratten } \\
{[154]}\end{array}$ & $\begin{array}{l}\text { Microwell with 3-electrode con- } \\
\text { figuration }\end{array}$ & $\begin{array}{l}\mathrm{Au} \mathrm{WE}+\mathrm{CE} \\
\mathrm{Ag} / \mathrm{AgCl} \mathrm{RE}\end{array}$ & $\begin{array}{l}\text { Glass } \\
\text { Polyimide }\end{array}+$ & $40 \mu m^{2} ; 0.6 \mathrm{~nL}$ & (Single) cell monitoring \\
\hline $\begin{array}{l}\text { Popovtzer } \\
{[168]}\end{array}$ & $\begin{array}{l}\text { Cylindrical microwell cell with } \\
\text { three electrodes }\end{array}$ & $\begin{array}{l}\mathrm{Au} \mathrm{WE}+\mathrm{CE}, \\
\mathrm{Ag} / \mathrm{AgCl} \mathrm{RE}\end{array}$ & $\begin{array}{l}\mathrm{Si} / \mathrm{SiO}_{2}+ \\
\mathrm{SU}-8\end{array}$ & $\begin{array}{l}30 \mu m^{2} \text { WE, } \quad 300 \mu m^{2} \\
\mathrm{CE}^{a} ; 100 \mathrm{~nL}\end{array}$ & $\begin{array}{l}\text { Toxicity detection in } \mathrm{H}_{2} \mathrm{O} \text { us- } \\
\text { ing e-coli }\end{array}$ \\
\hline $\begin{array}{l}\text { Baldwin } \\
{[170]}\end{array}$ & $\begin{array}{l}\text { 2-electrode electrochemical cell } \\
\text { positioned at outlet of elec- } \\
\text { trophoresis channel. }\end{array}$ & $\begin{array}{l}\mathrm{Pt} \text { WE, } \mathrm{Ag} / \mathrm{AgCl} \\
\mathrm{RE}\end{array}$ & glass & $40 \mu m$ wide, $1-2 m m$ long* & $\begin{array}{l}\text { Detection of dopamine after } \\
\text { electrophoretic separation }\end{array}$ \\
\hline Chen [169] & $\begin{array}{l}\text { Microwell with a single gold elec- } \\
\text { trode covering the entire well sur- } \\
\text { face. }\end{array}$ & $\mathrm{Au}$ WE & $\mathrm{Si} / \mathrm{SiO}_{2}$ & $\sim 0.79-5.15 \mathrm{pL}$ & $\begin{array}{l}\text { Detection of catecholamine } \\
\text { from individual cells }\end{array}$ \\
\hline Ball [166] & $\begin{array}{l}\text { Microwell with 2-electrode con- } \\
\text { figuration based on screen printing } \\
\text { techniques. }\end{array}$ & $\begin{array}{l}\text { Carbon WE, } \\
\mathrm{AgAgCl} \mathrm{RE} / \mathrm{CE}\end{array}$ & Ceramic & $16 \mathrm{~nL} *$ & $\begin{array}{l}\text { Demonstrate easy method to } \\
\text { fabricate electrodes in a mi- } \\
\text { crowell. }\end{array}$ \\
\hline $\begin{array}{l}\text { Lemay } \\
{[171]}\end{array}$ & $\begin{array}{l}\text { Nanometer sized square elec- } \\
\text { trodes inside a pyramid-shaped } \\
\text { well of } 310 \mathrm{~nm} \text { depth }\end{array}$ & $\mathrm{Au}$ & $\mathrm{Si} / \mathrm{SiO}_{2}$ & $225 n m^{2}-0.04 \mu m^{2}$ & $\begin{array}{l}\text { Demonstrate new fab. Method } \\
\text { for nanometer sized electrodes. }\end{array}$ \\
\hline $\begin{array}{l}\text { Vandaveer } \\
\text { [167] }\end{array}$ & $\begin{array}{l}\text { Redox cycling device based on a } \\
\text { microwell with two electrodes. }\end{array}$ & $\mathrm{Au}$ & $\mathrm{Si} / \mathrm{SiO}_{2}$ & $1.5-314 \mu m^{2} ; 2.5 \mathrm{pL}^{*}$ & $\begin{array}{l}\text { Study RC in a confined mi- } \\
\text { crowell of approx. } 2.5 \mathrm{pL}\end{array}$ \\
\hline Other & & & & & \\
\hline $\begin{array}{l}\text { Nissila } \\
{[156,172]}\end{array}$ & $\begin{array}{l}\text { EC conversions using photocat- } \\
\text { alytic properties of } \mathrm{TiO}_{2} \text {, with } \\
\text { ESI tip. }\end{array}$ & $\mathrm{TiO}_{2}$ & $\mathrm{Si} / \mathrm{SiO}_{2}$ & $0.5-4 \mu L$ analyte & $\begin{array}{l}\text { Mimic metabolic products of } \\
\text { CYP450 by photocatalytic ox- } \\
\text { idation }\end{array}$ \\
\hline $\begin{array}{l}\text { Zevenbergen } \\
{[153]}\end{array}$ & $\begin{array}{l}\text { Two facing planar electrodes with } \\
282 \mathrm{~nm} \text { spacing }\end{array}$ & $\mathrm{Pt}$ & $\mathrm{Si} / \mathrm{SiO}_{2}$ & $100-144 \mu m^{2} ; \sim 28 \mathrm{fL}^{*}$ & $\begin{array}{l}\text { Achieve sensitive detection of } \\
\sim 70 \text { molecules using RC }\end{array}$ \\
\hline
\end{tabular}

${ }^{a}$ This paper [168] reports square nm sized electrodes, but that seems very unlikely based on the fabrication methods and pictures reported in the paper. 
address the first issue. Lab-on-chip technology also offers the promise of a low-cost disposable chip, circumventing the need to clean electrodes after adsorption has occurred at the electrode surface. Electrochemical protein digestion is still a rather new approach with limited publications discussing this method. More research efforts are required both in the chemical and instrumental area to make it a useful method for the proteomics workflow.

Most miniaturized electrochemical cells that have been published are focused towards detection. A miniaturized electrochemical cell focused towards a good conversion efficiency requires a different cell design with sufficient electrode area. Some miniaturized flow-through cells have been reported, but are either limited in oxidation potential [156], or show a rather poor conversion efficiency while using still rather large internal cell volumes $[161,165]$. Moreover, very few flow-through cells that have been reported include a proper reference electrode [158]. Lastly, some of the flow-through cells discussed in the previous section are not suited for use in sensitive mass spectrometric detections because of the substrate materials used [157161, 164, 165]. E.g. PDMS, plexiglass and PET cannot withstand frequently used solvents like acetonitrile or formic acid [79, 82, 85].

Miniaturized electrochemical cells focused towards high conversion efficiencies can show unique electrochemical behavior, which makes it an interesting topic for electrochemical research. From a technological point of view, the facilities offered by the Mesa+ cleanroom and the BIOS lab-on-a-chip group provide the possibilities to make a smaller, more robust and better performing miniaturized electrochemical cell. In chapters 3 and 4, novel miniaturized cell designs are presented for the specific purpose of CYP450 based drug screening. Chapter 5 demonstrates the feasibility of using this design for electrochemical protein cleavage by some preliminary results. 


\section{Bibliography}

[1] N. C. Mishra. Introduction to Proteomics - Principles and applications. ISBN 0471754021. Wiley, 2010.

[2] D. Liebler. Introduction to Proteomics. ISBN 0-89603-992-7. Humana Press, 2002.

[3] N.-P. Website. http://nobelprize.org/ educational/medicine/ dna/index.html, 2010.

[4] P. James. Quarterly Review of Biophysics, 30:279-331, 1997.

[5] F. Crick. Nature, 227:561-563, 1970.

[6] A. J. Pellionisz. Cerebellum, 7:348-359, $2008 . \quad$ doi:10.1007/ s12311-008-0035-y.

[7] C. Mallery. http://henge.bio.miami.edu/ mallery/255/lectures/ lectures.htm, 2010.

[8] Wikipedia. http://www.wikipedia.org.

[9] J. van Mourik and J. van Dijk. Chemie voor het HBO - deel 1. Gohn Stafleu van Loghum, 1997.

[10] Y. Xiang, Y. Liu, and M. L. Lee. Journal of Chromatography A, 1104(1-2):198 - 202, 2006. ISSN 0021-9673. doi:10.1016/j.chroma.2005.11.118.

[11] K. Nokihara. Analytica Chimica Acta, 372(1-2):21 - 32, 1998. ISSN 00032670. doi:10.1016/S0003-2670(98)00347-X.

[12] J. Thomson. Proceedings of the Royal Society A, 89:1-20, 1913.

[13] C. E. Kolb and D. R. Herschbach. The Journal of Physical Chemistry, 88(20):4447-4451, 1984. doi:10.1021/j150664a001.

[14] P. Kebarle, Y. Ho, G. van Berkel, A. Bruins, et al. Fundamental aspects of electrospray ionization (ESI). In Cole [15], 1997.

[15] R. Cole (editor). Electrospray Ionization Mass Spectrometry. Wiley \& Sons, 1997.

[16] M. Wilm and M. Mann. Anal Chem, 68(1):1-8, 1996.

[17] X. Han, M. Jin, K. Breuker, and F. McLafferty. Science, 314:109-112, 2006.

[18] H. Permentier, U. Jurva, B. Barroso, and A. Bruins. Rap Comm in Mass Spectrometry, 17(14):1585-1592, 2003.

[19] H. P. Permentier and A. P. Bruins. Journal of the American Society for Mass Spectrometry, 15(12):1707 - 1716, 2004. ISSN 1044-0305. doi:10.1016/j. jasms.2004.09.003.

[20] J. Roeser, H. Permentier, A. Bruins, and R. Bischoff. Anal Chem, 82:75567565, 2010.

[21] J. Roeser, R. Bischoff, A. Bruins, and H. Permentier. Anal Bioanal Chem, 397:3441-3455, 2010. 
[22] N. Lion, T. Rohner, L. Dayon, I. Arnaud, E. Damoc, N. Youhnovski, Z. Wu, C. Roussel, J. Josserand, H. Jensen, J. Rossier, M. Przybylski, and H. Girault. Electrophoresis, 24(21):3533-3562, 2003.

[23] M. Prudent and H. H. Girault. Analyst, 134:2189-2203, 2009.

[24] S. Koster and E. Verpoorte. Lab on a Chip, 7:1394-1412, 2007.

[25] S. L. S. Freire and A. R. Wheeler. Lab on a Chip, 6(11):1415-1423, 2006. doi:10.1039/b609871a.

[26] A. J. de Mello. Lab on a Chip, 1:7N-12N, 2001.

[27] Z. Ouyang and G. Cooks. Annual Review of Analytical Chemistry, 2(1):187214, 2009. doi:10.1146/annurev-anchem-060908-155229.

[28] Z. Ouyang, R. J. Noll, and R. G. Cooks. Anal Chem, 81(7):2421-2425, 2009. doi:10.1021/ac900292w.

[29] J. Xie, Y. Miao, J. Shih, Y.-C. Tai, and T. D. Lee. Anal Chem, 77(21):69476953, 2005.

[30] P. Liuni, T. Rob, and D. J. Wilson. Rapid Communications In Mass Spectrometry, 24(3):315-320, 2010.

[31] A. Website. http://www.chem.agilent.com/en-us/products/ instruments/lc/analytical/systems/ 1200serieshplc-chipms/pages/default.aspx, 2010.

[32] B. Munos. Nat Rev Drug Discov, 8(12):959-968, 2009. ISSN 1474-1776.

[33] FDA. Guidance for industry, investigators, and reviewers - exploratory ind studies. http://www.fda.gov/cder/guidance/index.htm, 2006.

[34] J. A. Williams, R. Hyland, B. C. Jones, D. A. Smith, S. Hurst, T. C. Goosen, V. Peterkin, J. R. Koup, and S. E. Ball. Drug Metabolism and Disposition, 32(11):1201-1208, 2004. doi:10.1124/dmd.104.000794.

[35] L. Wienkers and T. Heath. Nat Rev Drug Discovery, 4:825-833, 2005.

[36] F. Guengerich. Chem Res Toxicol, 21:70-83, 2008.

[37] D. Richfield. Ph.D. thesis, Stellenbosch University, 2008.

[38] W. Lohmann and U. Karst. Analytical Bioanalytical Chemistry, 391:79, 2008.

[39] K. R. Williams and M. Wasilik. Journal of microelectromechnical systems, 12(6):761-778, 2003.

[40] P. A. Williams, J. Cosme, D. M. Vinkovic, A. Ward, H. C. Angove, P. J. Day, C. Vonrhein, I. J. Tickle, and H. Jhoti. Science, 305(5684):683-686, 2004. doi:10.1126/science.1099736.

[41] D. Nelson. Human Genomics, 4:59-65, 2009.

[42] F. Guengerich. FASEB J, 6:745-748, 1992.

[43] B. Akagah, A. T. Lormier, A. Fournet, and B. Figadere. Org Biomol Chem, 6(24):4494-4497, 2008. ISSN 1477-0520.

[44] U. Jurva, H. V. Wikstrom, and A. P. Bruins. Rapid Commun Mass Spectrom, 
16(20):1934-1940, 2002. ISSN 1097-0231.

[45] T. Johansson, L. Weidolf, and U. Jurva. Rap Comm Mass Spectrom, 21:23232331, 2007.

[46] A. Baumann and U. Karst. Expert Opin Drug Metab Toxicol, 6:715-731, 2010.

[47] A. Baumann, W. Lohmann, B. Schubert, H. Oberacher, and U. Karst. J of Chromatogr A, 1216:3192-3198, 2009.

[48] B. Blankert, H. Hayen, and J. Kaufmann. Electroanalysis, 17:1501-1510, 2005.

[49] P. Gamache, R. Smith, and I. Acworth. Spectroscopy, 18:14-24, 2003.

[50] T. Johansson, U. Jurva, G. Gronberg, L. Weidolf, and C. Masimirembwa. Drug Metabolism and Disposition, 37(3):571-579, 2009. doi:10.1124/dmd. 108.025171.

[51] U. Jurva, H. V. Wikstrom, and A. P. Bruins. Rap Comm in Mass Spectrometry, 14:529-533, 2000.

[52] U. Jurva, H. Wikstrom, L. Weidolf, and A. Bruins. Rap Comm Mass Spectrom, 17:800-810, 2003.

[53] U. Jurva, P. Bissel, and N. Castagnoli. J Am Chem Soc, 127:12368-12377, 2005.

[54] U. Jurva, A. Holmen, G. Groenberg, and L. Weidolf. Chem Res Toxicol, 21:928-935, 2008.

[55] S. van Leeuwen, B. Blankert, J. Kauffmann, and U. Karst. Analytical Bioanalytical Chemistry, 382:742-750, 2005.

[56] W. Lohmann and U. Karst. Analytical Bioanalytical Chemistry, 386:1701$1708,2006$.

[57] W. Lohmann and U. Karst. Anal Chem, 79:6831-6839, 2007.

[58] W. Lohmann, B. Meermann, and U. Karst. Anal Chem, 80:9769-9775, 2008.

[59] W. Lohmann and U. Karst. Anal Bioanal Chem, 394:1341-1348, 2009.

[60] W. Lohmann, R. Dotzer, G. Gutter, S. M. V. Leeuwen, and U. Karst. J Am Soc Mass Spectrom, 20:138-145, 2009.

[61] K. Madsen, J. Olsen, and U. Jurva. Chem Res Toxicol, 20:821-831, 2007.

[62] K. Madsen, C. Skonberg, and J. Olsen. Chem Res Toxicol, 21:1107-1119, 2008.

[63] K. Madsen, G. Gronberg, and J. Olsen. Chem Res Toxicol, 21:2035, 2008.

[64] T. Shono, T. Toda, and N. Oshino. Drug Metab Dispos, 9:481-482, 1998.

[65] K. Tahara, Y. Yano, and K. Kanagawa. Chem Pharm Bull, 55:1207-1212, 2007.

[66] K. Tahara and E. Makii. Analytical Science, 24:935-938, 2008.

[67] K. Tahara, T. Nishikawa, and Y. Abe. J Pharm Biomed Anal, 500:1030-1036, 
2009.

[68] M. Thevis, W. Lohmann, and W. Schanzer. Eur J Mass Spectrom, 14:163$170,2008$.

[69] E. Nouri-Nigjeh, H. P. Permentier, R. Bischoff, and A. P. Bruins. Analytical Chemistry, 82(18):7625-7633, 2010. doi:10.1021/ac101364s.

[70] S. Benetton, J. Kameoka, A. M. Tan, T. Wachs, H. Craighead, and J. D. Henion. Anal Chem, 75(23):6430-6436, 2003. doi:10.1021/ac030249+.

[71] S. R. Khetani and S. N. Bhatia. Nature Biotechnology, 26(1):120-126, 2008. doi:10.1038/nbt1361.

[72] G. J. Mahler, M. B. Esch, R. P. Glahn, and M. L. Shuler. Biotechnology and Bioengineering, 104(1):193-205, 2009. doi:10.1002/bit.22366.

[73] A. J. Bard and L. R. Faulkner. Electrochemical methods - fundamentals and applications. Wiley, 2nd edition, 2001.

[74] P. Regtien, F. van der Heijden, M. Korsten, and W. Olthuis. Measurement Science for Engineers. Kogan Page Science, 2004.

[75] D. J. Ives and G. J. Janz. Reference electrodes. Academic press, 1961.

[76] G. Burstein. Corrosion Science, 47(12):2858 - 2870, 2005. ISSN 0010938X. doi:10.1016/j.corsci.2005.07.002. A Century of Tafel's Equation: A Commemorative Issue of Corrosion Science.

[77] Z. Zhang, K. Yu, D. Bai, and Z. Zhu. Nanoscale Res Lett, 5:398-402, 2010.

[78] B. Park, N. Kitteringham, J. Maggs, M. Pirmohamed, and D. Williams. Annu Rev Pharmacol Toxicol, 45:177-202, 2000.

[79] R. Mukhopadhyay. Anal Chem, 79:3248-3253, 2007.

[80] M. A. Unger, H.-P. Chou, T. Thorsen, A. Scherer, and S. R. Quake. Science, 288:113-116, 2000.

[81] J. Liu, C. Hansen, and S. R. Quake. Anal Chem, 75:4718-4723, 2003.

[82] B.-Y. Kim, L.-Y. Hong, Y.-M. Chung, D.-P. Kim, and C.-S. Lee. Adv Funct Mater, 19:3796-3803, 2009.

[83] M. J. Madou. Fundamentals of Microfabrication. CRC Press, 2nd edition, 2002.

[84] R. M. Tiggelaar, F. Benito-Lopez, D. C. Hermes, H. Rathgen, R. J. Egberink, F. G. Mugele, D. N. Reinhoudt, A. van den Berg, W. Verboom, and H. J. Gardeniers. Chemical Engineering Journal, 131:163-170, 2007.

[85] Proplastics. http://www.proplasticsinc.com/chemical_resistance_guide.pdf, 2010.

[86] P. S. Nunes, P. D. Ohlsson, O. Ordeig, and J. P. Kutter. Microfluid Nanofluid, 9:145-161, 2010.

[87] J. Steigert, S. Haeberle, T. Brenner, C. Muller, C. P. Steinert, P. Koltay, N. Gottschlich, H. Reinecke, J. Ruhe, R. Zengerle, and J. Ducree. J Mi- 
cromechanics and Microengineering, 17:333-341, 2007.

[88] X. Illa, W. D. Malsche, J. Bomer, H. Gardeniers, J. Eijkel, J. Morante, A. Rodriguez, and G. Desmet. Lab on Chip, 9:1511-1516, 2009.

[89] D. A. Mair, M. Rolandi, M. Snauko, R. Noroski, F. Svec, and J. M. J. Frechet. Anal Chem, 79(13):5097-5102, 2007. doi:10.1021/ac070220w.

[90] J. Wang. Talanta, 56(2):223 - 231, 2002. ISSN 0039-9140. doi:10.1016/ S0039-9140(01)00592-6.

[91] W. R. Fawcett, M. Fedurco, Z. Kovacova, and Z. Borkowska. J Electroanal Chem, 368:265-274, 1994.

[92] A. Kraft. Int J Electrochem Sci, 2:355-385, 2007.

[93] G. Fiaccabrino, X.-M. Tang, N. Skinner, N. de Rooij, and M. Koudelka-Hep. Analytica Chimica Acta, 326:155-161, 1996.

[94] A. Chayahara, Y. Mokuno, Y. Horino, Y. Takasu, H. Kato, H. Yoshikawa, and N. Fujimori. Diamond and Related Materials, 13:1954-1958, 2004.

[95] M. Fryda, T. Matthee, S. Mulcahy, A. Hampel, L. Schafer, and I. Troster. Diamond and Related Materials, 12:1950-1956, 2003.

[96] C. Provent, W. Haenni, E. Santoli, and P. Rychen. Electrochimica Acta, 49(2223):3737 - 3744, 2004. ISSN 0013-4686. doi:10.1016/j.electacta.2004.02. 047.

[97] H. Kato, W. Futako, S. Yamasaki, and H. Okushi. Diamond and Related Materials, 14(3-7):340 - 343, 2005. ISSN 0925-9635. doi:10.1016/j.diamond. 2004.11.032.

[98] K. Soh, W. Kang, J. Davidson, Y. Wong, D. Cliffel, and G. Swain. Diamond and Related Materials, 17(4-5):900 - 905, 2008. ISSN 0925-9635. doi:10. 1016/j.diamond.2007.12.041.

[99] R. S. Kelly. Analytical Electrochemistry: The Basic Concepts. ASDLIB, http://www.asdlib.org/onlineArticles/ecourseware/Kelly_Potentiometry/ EC_CONCEPTS1.HTM, 2010.

[100] M. Shinwari, D. Zhitomirsky, I. Deen, P. R. Selvaganapathy, M. Deen, and D. Landheer. Sensors, 10:1679-1715, 2010.

[101] S. Yee, H. Jin, and L. Lam. Sens and Actuators, 15:337-345, 1988.

[102] R. Smith and D. Scott. IEEE Trans Biomed Eng, 2:83-90, 1986.

[103] A. van den Berg, A. Grisel, H. V. D. Vlekkert, and N. D. Rooij. Sens Actuators $B, 1: 425-432,1990$.

[104] H. Suzuki, A. Hiratsuka, S. Sasaki, and I. Karube. Sens and Actuators B, 46:104-113, 1998.

[105] H. Suzuki, T. Hirakawa, S. Sasaki, and I. Karube. Sens and Actuators B, 46:146-154, 1998.

[106] M. Ciobanu, J. P. Wilburn, N. I. Buss, P. Ditavong, and D. A. Lowy. Electro- 
analysis, 14(14):989-997, 2002. ISSN 1521-4109.

[107] K. Lill and A. Hassel. J Solid State Electrochem, 10:941-946, 2006.

[108] M. Kunimatsu, H. Qiao, and T. Okada. Journal of the electrochemical society, 152:E161-E166, 2005. doi:10.1149/1.1874732.

[109] P. K. J. H. D. Hubbard. Sens and Actuators B, 22:13-26, 1994.

[110] I.-Y. Huang, R.-S. Huang, and L.-H. Lob. Sens and Actuators B, 94:53-64, 2003.

[111] J. Ha, S. M. Martin, Y. Jeon, I. J. Yoon, R. B. Brown, H. Nam, and G. S. Cha. Analytica Chimica Acta, 549(1-2):59 - 66, 2005. ISSN 0003-2670. doi:10.1016/j.aca.2005.06.011.

[112] L. Bousse, P. Bergveld, and H. Geeraedts. Sens and Actuators, 9:179-197, 1986.

[113] R. Goffe and A.C.Tseung. Med \& Biol Eng \& Comput, 16:670-676, 1978.

[114] R. Mihajlovic, Z. Simic, L. Mihajlovic, A. Jokic, M. Vukasinovic, and N. Rakicevic. Analytica Chimica Acta, 318:287-295, 1996.

[115] R. Tiggelaar. Ph.D. thesis, University of Twente, 2004.

[116] D. Midgley. Talanta, 37(8):767-781, 1990. ISSN 0039-9140.

[117] R. Frohlich, A. Rzany, J. Riedmuller, A. Bolz, and M. Schaldach. Journal of Materials Science: Materials in Medicine, 7:393-397, 1996.

[118] A. Barbu and V. Plichon. Electrochimica Acta, 42:489-492, 1996.

[119] S. Negi, R. Bhandari, L. Rieth, and F. Solzbacher. Sensors and Actuators B: Chemical, 137:370-378, 2008. ISSN 0925-4005.

[120] K. Nishio, Y. Watanabe, and T. Tsuchiya. Thin Solid Films, 350(1-2):96-100, 1999. ISSN 0040-6090.

[121] M. A. El Khakani and M. Chaker. Thin Solid Films, 335(1-2):6-12, 1998. ISSN 0040-6090.

[122] R. S. Chen, Y. S. Huang, Y. M. Liang, D. S. Tsai, and K. K. Tiong. Journal of Alloys and Compounds, 383(1-2):273-276, 2004. ISSN 0925-8388.

[123] R.-S. Chen, Y.-S. Huang, Y.-M. Liang, D.-S. Tsai, Y. Chi, and J.-J. Kai. Journal of Materials Chemistry, 13:2525-2529, 2003.

[124] S. Yao, M. Wang, and M. Madou. Journal of the electrochemical society, 148:H29-H36, 2001.

[125] B. Wessling. Ph.D. thesis, Technischen Hochschule Aachen, 2007.

[126] J. Hendrikse, W. Olthuis, and P. Bergveld. Sensor and Actuators B, 47:1-8, 1998.

[127] E. Slavcheva, R. Vitushinsky, W. Mokwa, and U. Schnakenberg. J Electrochem Soc, 151:E226-E237, 2004.

[128] W. Olthuis, M. Robben, P. Bergveld, M. Bos, and W. van der Linden. Sens Actuators B, 4:247-256, 1990. 
[129] M. Huppauff and B. Lengeler. Journal of the electrochemical society, 140:598-602, 1993.

[130] H. Yang, S. K. Kang, C. A. Choi, H. Kim, D.-H. Shin, Y. S. Kim, and Y. T. Kim. Lab on Chip, 4:42-46, 2004.

[131] R. Franklin, M. Johnson, K. Scottt, J. H. Shim, H. Nam, D. Kipket, and R. Brown. In Sensors, 2005 IEEE, page 4 pp. 2005. doi:10.1109/ICSENS. 2005.1597971.

[132] C. Schabmueller, D. Loppow, G. Piechotta, B. Schutze, J. Albers, and R. Hintsche. Biosensors and Bioelectronics, 21(9):1770-1776, 2006. ISSN 0956-5663.

[133] C. O. Parker, Y. H. Lanyon, M. Manning, D. W. M. Arrigan, and I. E. Tothill. Anal Chem, 81(13):5291-5298, 2009. doi:10.1021/ac900511e.

[134] F.-R. F. Fan and A. J. Bard. Science, 267(5199):871-874, 1995.

[135] G. Diehl, A. Liesener, and U. Karst. Analyst, 126:288-290, 2001. doi:10. 1039/b009064f.

[136] H. Deng and G. J. V. Berkel. Electroanalysis, 11(12):857-865, 1999.

[137] G. Hambitzer, J. Heitbaum, and I. Stassen. Anal Chem, 70:838-842, 1998. doi:10.1021/ac970753c.

[138] M. C. S. Regino and A. Brajter-Toth. Anal Chem, 69:5067-5072, 1997. doi: $10.1021 / \mathrm{ac} 961261 \mathrm{n}$.

[139] X. Xu, W. Lu, and R. B. Cole. Anal Chem, 68:4244-4253, 1996. doi:10.1021/ ac960362i.

[140] G. J. V. Berkel, J. Quirke, R. Tigani, A. Dilley, and T. Covey. Anal Chem, 70:1544-1554, 1998. doi:10.1021/ac971348o.

[141] H. Hayen and U. Karst. Anal Chem, 75:4833-4840, 2003.

[142] W. Lohmann, H. Hayen, and U. Karst. Anal Chem, 80:9714-9719, 2008.

[143] K. J. Volk, M. S. Lee, R. A. Yost, and A. Brajter-Toth. Anal Chem, 60:720$722,1988$.

[144] K. J. Volk, R. A. Yost, and A. Brajter-Toth. Anal Chem, 61(15):1709-1717, 1989. ISSN 0003-2700.

[145] A. Blazewicz, Z. Fijalek, M. Warowna-Grzeskiewicz, and M. Boruta. Journal of Chromatography A, 1149(1):66-72, 2007.

[146] H. Deng and G. V. Berkel. Anal Chem, 71:4284-4293, 1999. doi:10.1021/ ac990527y.

[147] A. Arribas, M. Moreno, E. Bermejo, M. Lorenzo, A. Zapardiel, and M. Chicharro. Electrophoresis, 30:3480-3488, 2009.

[148] M. Morita, M. L. Longmire, and R. W. Murray. Anal Chem, 60(24):27702775, 1988.

[149] B. Lakard, J. Jeannot, M. Spajer, G. Herlem, M. de Labachelerie, P. Blind, 
and B. Fahys. Electrochimica Acta, 50:1863-1869, 2005.

[150] M. E. Sandison and J. M. Cooper. Lab on Chip, 6:1020-1025, 2006.

[151] C. G. Zoski and N. Simjee. Anal Chem, 76:62-72, 2004.

[152] H. Suzuki, T. Hirakawa, S. Sasaki, and I. Karube. Analytica Chimica Acta, 387(1):103-112, 1999.

[153] M. Zevenbergen, D. Krapf, M. Zuiddam, and S. Lemay. Nano Lett, 7(2):384388, 2007.

[154] C. Bratten, P. Cobbold, and J. Cooper. Anal Chem, 69(2):253-258, 1997.

[155] T. Li, L. Su, W. Hu, H. Dong, Y. Li, and L. Mao. Anal Chem, 82:1521-1526, 2010.

[156] T. Nissila, L. Sainiemi, M.-M. Karikko, M. Kemell, M. Ritala, S. Franssila, R. Kostiainen, and R. Ketola. In Microtas conference, W72F. 2009.

[157] H. Suzuki and R. Yoneyama. Sensors and Actuators B: Chemical, 96(1-2):3845, 2003.

[158] S. Kim, H. Lim, T. Chung, and H. Kim. Sens Actuators B, 115:212-219, 2006.

[159] J. S. Rossier, M. A. Roberts, R. Ferrigno, and H. H. Girault. Anal Chem, 71:4294-4299, 1999.

[160] K. Ueno, H. Kim, and N. Kitamura. Anal Chem, 75(9):2086-2091, 2003.

[161] J. Hranicek, V. Cerveny, and P. Rychlovsky. Cent Eur J Chem, 7:675-682, 2009.

[162] S. Bohm, D. Pijanowska, W. Olthuis, and P. Bergveld. Biosensors and Bioelectronics, 16:391-397, 2001.

[163] E. Sahlin, A. ter Halle, K. Schaefer, J. Horn, M. Then, and S. G. Weber. Anal Chem, 75:1031-1036, 2003.

[164] J. S. Rossier, C. Vollet, A. Carnal, G. Lagger, V. Gobry, H. H. Girault, P. Michel, and F. Reymond. Lab on Chip, 2:145-150, 2002.

[165] G. Liljegren, A. Dahlin, C. Zettersten, J. Bergquista, and L. Nyholm. Lab on a Chip, 5:1008, 2005.

[166] J. C. Ball, D. L. Scott, J. K. Lumpp, S. Daunert, J. Wang, and L. G. Bachas. Anal Chem, 72:497-501, 2000.

[167] W. R. Vandaveer, D. J. Woodward, and I. Frits. Electrochimica Acta, 48:33413348, 2003.

[168] R. Popovtzer, T. Neufeld, D. Biran, E. Z. Ron, J. Rishpon, and Y. ShachamDiamand. Nano Lett, 5:1023-1027, 2005.

[169] P. Chen, B. Xu, N. Tokranova, X. Feng, J. Castracane, and K. D. Gillis. Anal Chem, 75:518-524, 2003.

[170] R. P. Baldwin, T. J. Roussel, M. M. Crain, V. Bathlagunda, D. J. Jackson, J. Gullapalli, J. A. Conklin, R. Pai, J. F. Naber, K. M. Walsh, and R. S. Keyn- 
ton. Anal Chem, 74:3690-3697, 2002.

[171] S. G. Lemay, D. M. van den Broek, A. J. Storm, D. Krapf, R. M. M. Smeets, H. A. Heering, and C. Dekker. Anal Chem, 77:1911-1915, 2005.

[172] L. Sainiemi, T. Nissila, V. Jokinen, T. Sikanen, T. Kotiaho, R. Kostiainen, R. A. Ketola, and S. Franssila. Sensors and Actuators B: Chemical, 132(2):380 - 387, 2008. ISSN 0925-4005. doi:10.1016/j.snb.2007.09.077. Transducers '07/Eurosensors XXI. 


\section{A chip for conversions in drug metabolism studies}

In this chapter, a design of a microfluidic microreactor chip for electrochemical conversion of analytes, containing a palladium reference electrode and platinum working and counter electrodes is described. The counter electrode is placed in a separate side-channel on chip to prevent unwanted side-products to appear in the measured spectrograms. Using this chip cyclic voltammograms are measured in volumes of 9.6nL. Furthermore the conversion efficiency of ferricyanide is characterized using UV/vis-spectroscopy. An on-line conversion of $97 \%$ is obtained using a flow rate of $1 \mu \mathrm{L} / \mathrm{min}$. The microreactor chip is used to study the electrochemical metabolism pathway of amodiaquine using electrochemistry (EC)/ liquid chromatography (LC)/ mass spectrometry $(M S)$. The results are compared with measurements obtained with commercially available electrochemical flow-through cells. Using the chip it was possible to obtain similar results. Therefore, we have fabricated an electrochemical cell on-chip which is used successfully in EC-UV/vis and EC-LC-MS experiments. ${ }^{1}$

\footnotetext{
${ }^{1}$ Modified from: a paper published in Lab-on-Chip [1]. The original paper is written in close cooperation with the Institute of Inorganic and Analytical Chemistry of the University of Münster. Co-authors are A. Baumann, U. Karst, F.T.G. van den Brink, W. Olthuis and A. van den Berg.
} 


\subsection{Introduction}

Electrochemisty (EC) is well-known in analytical chemistry as a useful addition to techniques like liquid chromatography (LC) and mass spectrometry (MS). In traditional LC-MS experiments, EC can be used to convert non-polar species to more polar reaction products and thus significantly increase the ionization efficiency in electrospray sources [2-5]. This can be performed by integrating EC into the electrospray interface, but also inside a separate on-line flow cell which is placed directly in front of the interface. The latter gives the advantage of superior control of the reaction conditions, and allows a higher conversion of the electrochemically active species [6].

Further examples of the coupling of EC to MS and/or LC can be found in a variety of studies in biomedical and also environmental analysis. In these studies the electrochemical cell is used successfully for mimicking oxidative reactions in drug metabolism studies $[5,7]$. These reactions are catalysed in the organism by enzymes of the cytochrome P450 family. Bruins et al. [8] mimicked the oxidations using a commercially available coulometric flow-through cell from ESA Bioscience Inc. (Chelmsford, MA). Also, using the same cell in an EC-LC-thermospray-MS system, the enzymatic and electrochemical oxidation pathways of uric acid are compared [9].

Furthermore, Karst et al. used the ESA cell to study the electrochemical metabolism pathway of clozapine, an antipsychotic drug [10], and amodiaquine, a known antimalarial agent [11]. Another interesting application of EC-MS is the tyrosine specific protein cleavage reported by Permentier et al. [12].

The coulometric cells from ESA contain a three-electrode set-up including a reference electrode of the $\alpha$-hydrogen/ palladium type. The potential of this pseudoreference electrode is only stable under certain conditions like a known, constant $\mathrm{pH}$ [13]. Other disadvantages are the relatively large volume and that it can not be disassembled for maintenance or electrode replacement. Besides the flow-through cell, different types of wall-jet cells are commercially available.

Other companies that produce electrochemical cells are BioAnalytical Systems and Antec Leyden [14]. Their cells are based on the thin-layer flow concept. Those cells require lower flow rates for an efficient conversion, but bear the advantage of various replaceable and interchangeable electrode materials.

Besides the already mentioned commercially available cells, various papers are published in which home-made flow-through cells are combined with mass spectrometry 
[15, 16]. Back in 1971, Bruckenstein et al. [17] already coupled a porous glassy carbon electrode with an MS to study the gaseous reaction products $\mathrm{NO}$ and $\mathrm{N}_{2} \mathrm{O}$ of the reduction of $\mathrm{NO}_{2}^{-}$.

Hambitzer and Heitbaum [18] were the first in 1986 to construct a two-electrode thinlayer flow cell to connect with a MS to study the oxidation of N,N-dimethylaniline. They employed a glass frit to avoid mixing of electrolyte between counter and working electrode compartments. The reference electrode was placed in a separate flask, connected to the flow cell via a luggin capillary.

Cole et al. minimized the time between the electrochemical conversion of species and detection in the MS, to detect short-living radicals using a home-made, lowvolume, three-electrode flow-cell $[19,20]$. They made a cell in a coaxial-like configuration, consisting of an inner platinum core partly covered with a fused-silica layer which is positioned inside an auxiliary outer electrode. The latter electrode was also acting as spray needle.

In the configurations discussed so far, EC is used as conversion tool for subsequent detection in MS. However, EC can also be used as detector for various compounds like ammonia, lactate, glucose, dopamine and glutamate [21-24].

Our goal is to develop an electrochemical cell in a lab-on-chip, specifically aimed towards high electrochemical conversions for (on-line) use with mass spectrometry in drugs screening. Also, an extra sensing electrode is placed after the conversion area to detect generated species.

We have developed a microfluidic chip with a fast and high conversion rate of the introduced species. This chip, with a main channel volume of only $9.6 \mathrm{~nL}$, is fabricated out of glass for its inert nature. A three electrode set-up is integrated into the chip. The chip contains a palladium pseudo-reference electrode and platinum working and counter electrodes. The counter electrode is placed in a separate side-channel on the chip to prevent unwanted side-products to appear in the measured spectra.

Cyclic voltammograms were recorded to characterize the EC cell. As two test cases, EC-UV/vis measurements on the conversion of ferricyanide and EC-LC-MS measurements on the oxidative metabolism of amodiaquine, are included to illustrate the performance of our cell in practical applications.

Compared to the commercially available cells, our chip is particularly useful in cases where only limited sample volumes are available. In addition, it is possible to use the extra sensing electrode present on the chip to perform immediate electrochemical detection after conversion. Also, future integration of this design together with liquid chromatography on chip [25] is possible. 


\subsection{Theory and design considerations}

For drug screening it is important to obtain total conversion of the inserted chemical species of interest. Factors that influence the conversion efficiency are, among others, the dimensions of the cell and the volumetric flow velocity. From a microfabrication point of view, the most easy way to incorporate electrodes into a small cell is to place these electrodes at the bottom. A schematical overview of such a cell is given in figure 3.1.

It is assumed that the length (l) of the working electrode is much larger than the width (w), which is in turn much larger than the height of the channel (h). Furthermore the flow is assumed to be laminar and the fluids are incompressible. Under these assumptions the velocity flow profile in $\mathrm{x}$-direction of the channel can be described by the following expression [26]:

$$
v_{x}=v_{\max } \cdot\left(1-\frac{4 y^{2}}{h^{2}}\right)
$$

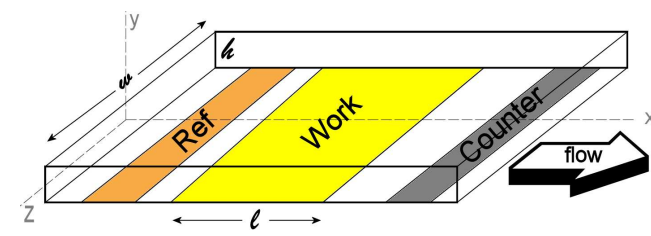

Figure 3.1: Typical thin-layer flow cell with electrodes placed at the bottom of the channel.

We assume this flow to be valid for every value of $\mathrm{z}$ inside the channel. By doing so, we make a small error at the vertical walls perpendicular to the plane of the working electrode. Since we assume the width of the channel to be much larger than the height, this error is negligible. The volumetric flow velocity is equal to the integral of the flow velocity, multiplied by the channel width:

$$
Q=w \cdot \int_{-h / 2}^{h / 2} v_{x} d y
$$

In a worst-case scenario, the minimum residence time of an ion inside the channel above the working electrode is equal to: 


$$
t_{r}=\frac{l}{v_{\max }}
$$

Of course, the actual residence time will be longer on average. Since our goal is to obtain total conversion, a larger residence time inside the channel is beneficial for the conversion rate.

In order to obtain a good conversion efficiency, the height (h) of the channel must be smaller or equal to the diffusion length of ions in the y-direction $\left(y_{d} i f f\right)$ within the residence time. The following expression describes the relation between the diffusion length and the residence time and the consequences for the allowed channel height [27]:

$$
y_{\text {diff }}=\sqrt{4 \cdot D \cdot t_{r}} \quad h \leq y_{\text {diff }}
$$

The hydraulic channel resistance of a rectangular shaped channel is equal to [26]:

$$
R_{f}=\frac{P}{Q}=\frac{12 \cdot \eta \cdot l}{h^{3} \cdot w}
$$

Note that the parameter length (1) in equation 3.5 is only the length of the channel containing the working electrode. Therefore the pressure $(\mathrm{P})$ describes only the pressure drop over that part of the channel. The same remark is valid for the hydraulic resistance.

Finally, a result for the pressure difference $(\mathrm{P})$ over the part of the channel containing the working electrode is obtained as a function of the viscosity $(\eta), \mathrm{h}, \mathrm{l}, \mathrm{w}$ and $\mathrm{Q}$ if equation 3.1-3.5 are combined:

$$
P=\frac{81 \cdot \eta \cdot Q^{4}}{128 \cdot D^{3} \cdot l^{2} \cdot w^{4}}
$$

To prevent problems with fluidic interconnects, we limit the pressure drop over the part of the channel containing the working electrode to $1 \cdot 10^{5} \mathrm{~Pa}$ (1 bar). To obtain a 
a)

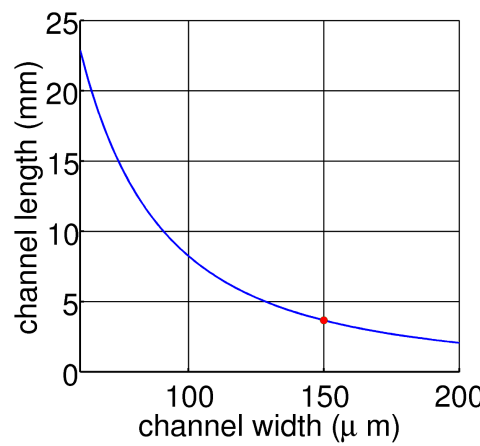

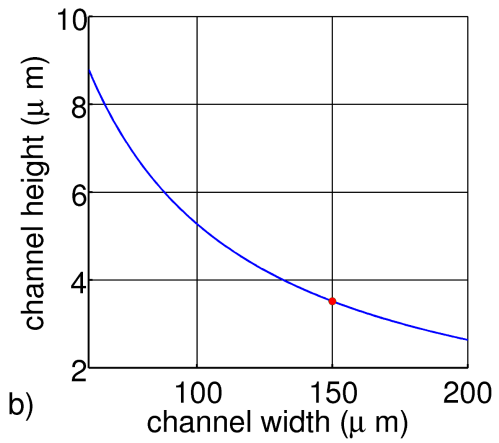

Figure 3.2: Channel length (a) and height (b) as a function of the channel width, using equations 3.1-3.6 and $P=1 \cdot 10^{5} \mathrm{~Pa}, \eta=8.9 \cdot 10^{-4} \mathrm{~Pa} \cdot \mathrm{s}, Q=10^{-6} \mathrm{~L} / \mathrm{min}$, $D=4 \cdot 10^{-11} \mathrm{~m}^{2} / \mathrm{s}$.

good interface with the surrounding equipment the volumetric flow velocity is aimed to be $1 \mu \mathrm{l} / \mathrm{min}$. Also, the diffusion constant of the protein inside the channel is assumed to be $4 \cdot 10^{-11} \mathrm{~m}^{2} / \mathrm{s}$ and the viscosity of the solvent is equal to the viscosity of water at room temperature $\left(8.9 \cdot 10^{-4} \mathrm{~Pa} \cdot \mathrm{s}\right)$. Using equation 3.1-3.6, it is now possible to calculate the channel length containing the working electrode and the channel height as a function of the channel width.

The results are shown in figure 3.2. From figure 3.2a it can be seen that the channel length is decreasing for larger channel widths. Also from figure $3.2 \mathrm{~b}$ it can be seen that the channel height is decreasing for larger channel widths. We can explain this by the time the ion needs to diffuse towards the working electrode, which is increasing for larger channel heights. To compensate for this effect the channel length needs to be enlarged to increase the residence time of the ions inside the channel.

The final dimensions of the main channel of the chip are $4 \mu \mathrm{m}$ by $150 \mu \mathrm{m}$ by $16 \mathrm{~mm}$ (h, w, l). The selected length is four times larger than the values calculated in this section, to realize a conservative design in terms of good conversion efficiency. Therefore, the cell can handle even slower diffusing species. As a result, the total pressure over the main channel is around $5 \cdot 10^{5} \mathrm{~Pa}(5 \mathrm{bar})$, which is still low enough to prevent problems with the fluidic interconnects. Two different designs have been made; one design with the working electrode placed over the entire bottom surface of this channel and one design with the working electrode placed over $75 \%$ of the bottom channel surface. 


\subsection{Experimental}

\subsubsection{Chip design and fabrication}

A schematic overview of the different components of the chip, along with the final result is shown in figure 3.3.

a)

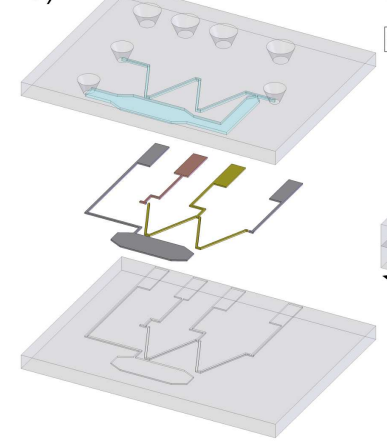

b)

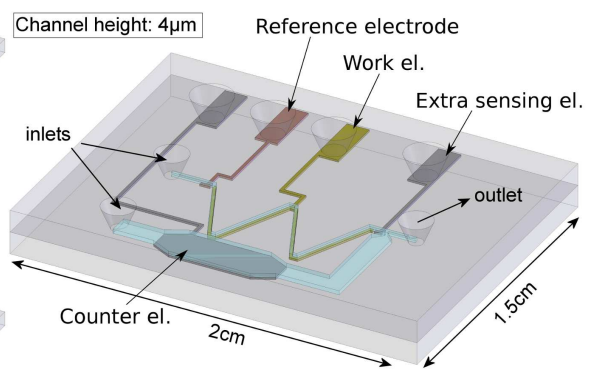

C)

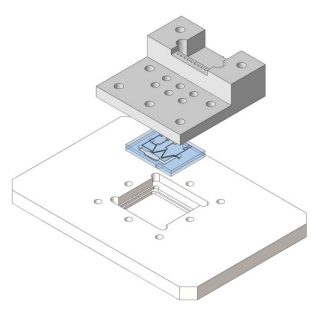

Figure 3.3: Overview of the fabricated chip. a) Bottom layer with recess etched wells for the electrodes and top wafer with inlets and fluidic channels. b) Assembled view of the total chip $(1.5$ by $2 \mathrm{~cm})$ and the different electrodes and inlets indicated. c) Chip and chipholder.

This chip is fabricated out of two glass (Pyrex) wafers. On the bottom wafer photoresist is patterned using standard lithography. Wells for the first electrode are etched (150nm deep) using buffered HF (BHF). Next, a palladium electrode is sputtered and patterned by lift-off. The general electrode layer thickness is $140 \mathrm{~nm}$. For the platinum electrodes, the photolithography - BHF etch - sputtering - lift-off cycle, is repeated. In total four electrodes are present on the chip: one pseudo reference electrode made of palladium, and three platinum electrodes used as working, counter and extra sensing electrode. The counter electrode is placed in a separate side-channel to prevent unwanted reaction products to appear into the measured signal.

On the backside of the top wafer, first a chromium/gold layer is applied and patterned by lithography and a wet gold / chromium etch. This gold layer is acting as mask for the fluidic channels, which are wet etched using BHF into the backside of the top wafer.

Fluidic inlets and outlets, along with access holes for the electrode pads are powderblasted into the top wafer from the top side. As a last step, the two wafers are 
bonded together using a press (Carver Inc, model 4386, applied pressure $12.7 \mathrm{MPa}$ ) and an annealing treatment of $450^{\circ} \mathrm{C}$ for 1 hour. More details on the chip fabrication can be found in the appendix of this thesis.

During experiments the chip is placed inside an in-house developed chip holder to provide easy interconnects to the chip (see fig 3.3c). Fluidic connections are provided by nanoport nuts (Upchurch Scientific). Electric contacts to the chip are made using spring metal contact probes (ISI Interconnect Specialities Inc.).

\subsubsection{Cyclic voltammetry and conversion efficiency study}

The set-up used for the cyclic voltammetry measurements and the conversion efficiency study is shown in figure 3.4. A syringe pump (Harvard Apparatus, PHD $22 / 2000)$ is connected through fused glass capillary $(100 \mu \mathrm{m}$ inner diam.) to a switchable valve (Upchurch Scientific, V-1241-DC). This valve is connected to the inlets of the chip and bottles containing the sample solution, demineralized water and a waste container. Electrochemical measurements are recorded using a bipotentiostat (Bio-Logic SAS, type BiStat).

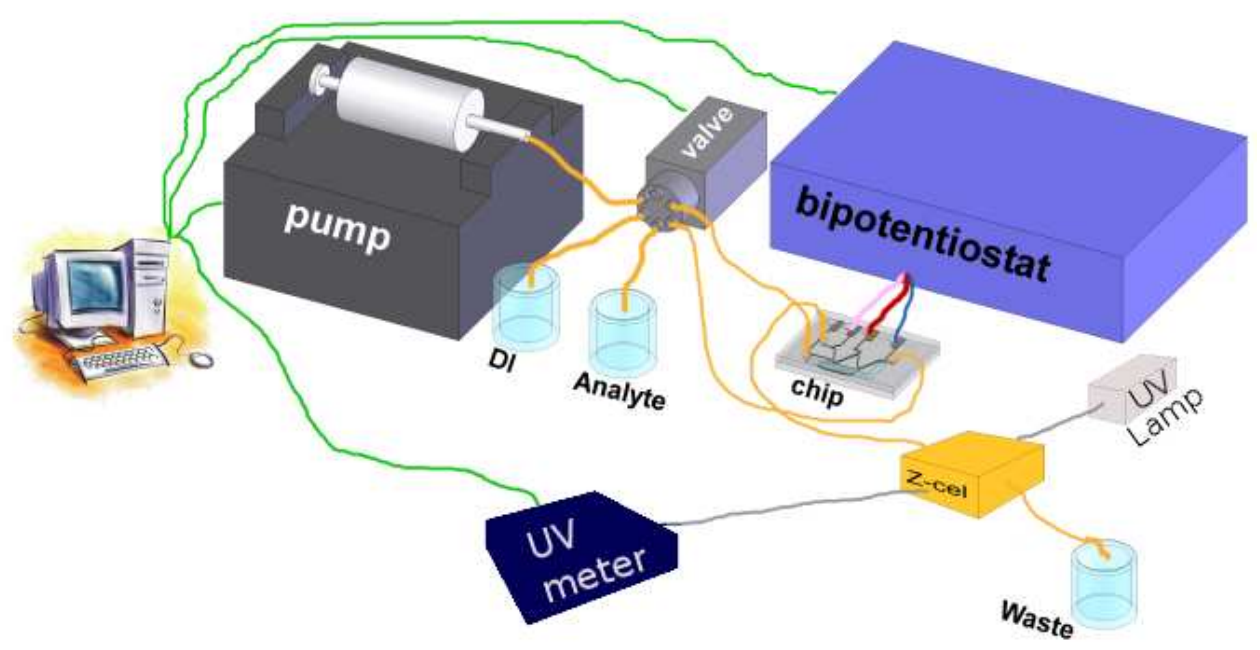

Figure 3.4: Setup used in the cyclic voltammetry and conversion efficiency measurements.

For the UV/vis measurements the outlet of the chip is connected to a z-cell with an optical pathlength of $5 \mathrm{~mm}$ and internal volume of approx. $1 \mu \mathrm{L}$ (Zeutec OptoElektronic $\mathrm{GmbH}$, Micro flow cell). Inside this z-cell UV/vis adsorbance measurements are recorded using a deuterium light source (Ocean Optics, DT-MINI-2-GS 
Mikropack) and an UV/vis spectrometer (Ocean Optics, HR4000). The fluidic and electrochemical part of the set-up is controlled by a Labview application. UV/vis measurements are recorded using software from Ocean Optics.

In general, the set-up is operated using the following protocol. First the whole set-up including the chip is rinsed with $100 \mu \mathrm{L}$ of DI water. Next, the sample solution is infused into the side channel of the chip. After five minutes, the flow is switched to the main channel. Again after five minutes, the electrochemical measurements are started.

For the cyclic voltammetry measurements, the potential is varied between -0.4 and $0.4 \mathrm{~V}$ with a scan rate of $20 \mathrm{mV} / \mathrm{s}$. For the conversion efficiency study a constant potential of $-0.5 \mathrm{~V}$ or $0.5 \mathrm{~V}$ is applied for several minutes until the measured spectrogram was stable. All potentials given in this chapter are defined vs. the palladium pseudo-reference electrode with the $\mathrm{pH}$ in all measurements held constant at 7 .

\subsubsection{Amodiaquine study}

In 2007, Lohmann and Karst published a paper describing the electrochemical generation and identification of reactive metabolites of amodiaquine [11]. The set-up used for this study consists of a flow-through cell for electrochemical oxidation from ESA Bioscience Inc. (Chelmsford, MA), liquid chromatography for separation and mass spectrometry for the detection. A similar set-up was applied to test the performance of our chip system. The flow-through cell in the original set-up is exchanged by the chip and for comparison purposes, a commercial wall-jet cell was applied.

The set-up used in this experiment is shown in figure 3.5. Sample solution is introduced into either the chip or a commercial flow cell (Antec Leyden, Flexcell) using two syringe pumps. The pump connected to the side channel of the chip was switched off during electrochemical oxidation.

The oxidation products are collected in a $10 \mu \mathrm{L}$ sample loop, placed in a 6-port valve. After filling the sample loop, the valve is switched so the analytes are flushed onto the HPLC column and the MS subsequently. Due to the difference in volume between chip and sample loop, this setup can only be used for applications which are not time specific. For future, time critical EC-MS experiments the chip can be extended with an electrospray injection needle as described in a paper by M. Brivio et al. [28].

\subsubsection{Instrumentation}

Two syringe pumps from Cole Parmer (Vernon Hills, IL, type 74900) were used to introduce analyte into the chip. The potentials for the chip were applied by an in-house developed potentiostat. 


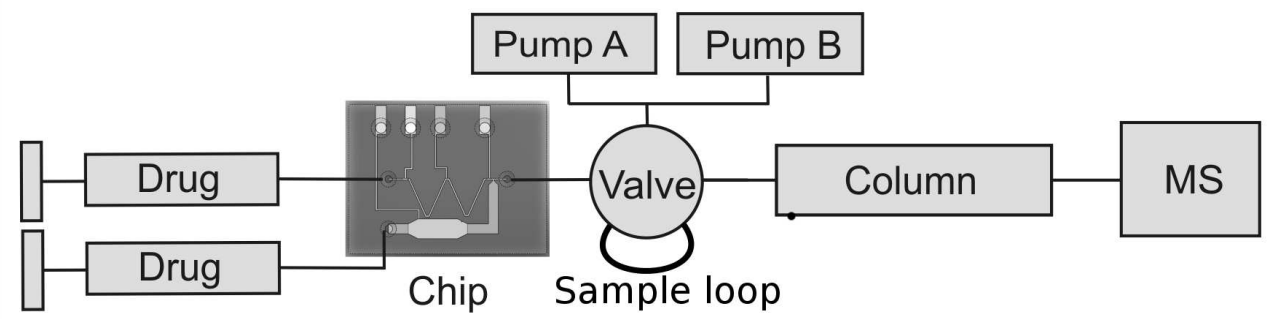

Figure 3.5: Setup used for the amodiaquine experiment (Electric connections and potentiostat not shown).

For the EC/LC/MS measurements, an LC system from Antec Leyden (Zoeterwoude, The Netherlands) was used, consisting of two LC 110 HPLC pumps, an AS 100 autosampler and a Decade II electrochemical detector, equipped with a Flexcell including a platinum working electrode.

The cells were coupled to a microTOF mass spectrometer from Bruker Daltronics (Bremen, Germany) equipped with an electrospray ionization (ESI) source.

The software used for controlling the EC-LC system was the Alexys data system (Antec Leyden). For controlling the MS and MS data handling microTOF Control v1.1 and DataAnalysis V3.3 (Bruker Daltronics) were used.

\subsubsection{Chemicals}

All reagents used were at least of of analytical reagent grade and obtained from Sigma Aldrich Chemie GmbH (Steinheim, Germany). Demineralized water used for the solutions was provided by a water purification system (Milli-Q Synthesis A10, Millipore). A stock solution was used for the Cyclic Voltammetry measurements, containing a mixture of $2.5 \mathrm{mM}$ potassiumferricyanide, $2.5 \mathrm{mM}$ potassiumferrocyanide, $0.1 \mathrm{M}$ potassiumnitrate as supporting electrolyte and $2 \mathrm{mM}$ phosphate buffer ( $\mathrm{pH} 7)$.

The solution used for EC-UV/vis experiment contained a mixture of $1 \mathrm{mM}$ potassiumferricyanide, $1 \mathrm{mM}$ potassiumferrocyanide, $0.5 \mathrm{M}$ potassiumnitrate and $2 \mathrm{mM}$ phosphate buffer ( $\mathrm{pH} 7)$. UV/vis calibration has been done using different solutions containing a range of potassiumferricyanide concentrations from $0.01 \mathrm{mM}$ to $2 \mathrm{mM}$. For the amodiaquine (AQ) experiments, $50 \mu \mathrm{M}$ AQ was dissolved in $20 \mathrm{mM}$ ammonium formate solution with $\mathrm{pH}$ adjusted to 7.4. 
Table 3.1: Measured current at $-0.4 \mathrm{~V}$ and various flow rates using two chips with different working electrode size.

\begin{tabular}{llllll}
\hline Chip & WE size & $0 \mu \mathrm{L}$ & $0.1 \mu \mathrm{L}$ & $0.25 \mu \mathrm{L}$ & $0.5 \mu \mathrm{L}$ \\
\hline 1 & $2.4 m m^{2}$ & $-0.15 \mu \mathrm{A}$ & $-0.37 \mu \mathrm{A}$ & $-0.70 \mu \mathrm{A}$ & $-1.25 \mu \mathrm{A}$ \\
2 & $1.8 m m^{2}$ & $-0.15 \mu \mathrm{A}$ & $-0.39 \mu \mathrm{A}$ & $-0.72 \mu \mathrm{A}$ & $-1.25 \mu \mathrm{A}$
\end{tabular}

\subsection{Results and discussion}

\subsubsection{Cyclic voltammetry measurements}

On-line, on-chip cyclic voltammetry measurements are recorded at various flow rates. The results for one specific type of chip are shown in figure 3.6.
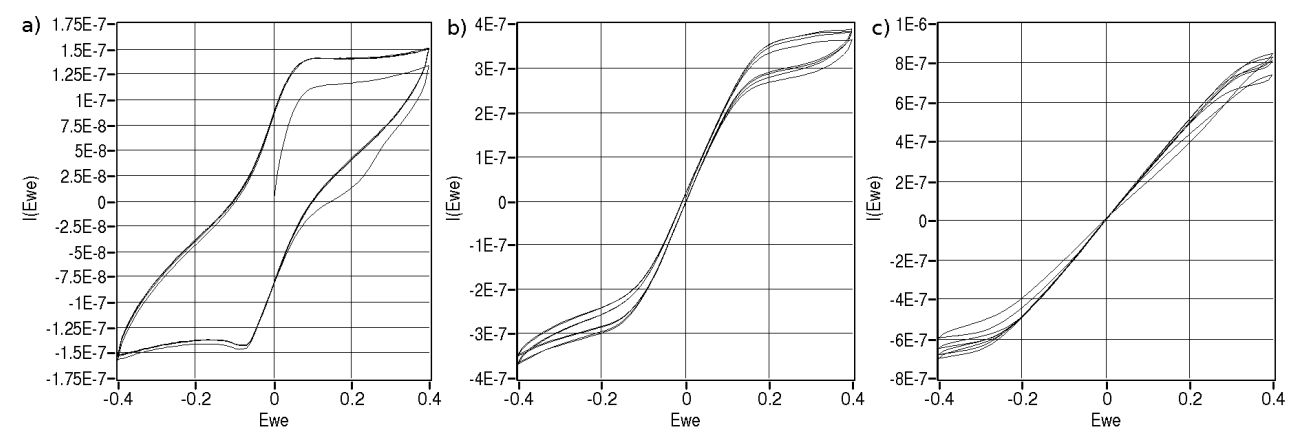

Figure 3.6: Cyclic voltammograms recorded in a solution containing 2.5mM ferrocyanide, 2,5mM ferricyanide, 0.1M $\mathrm{KNO}_{3}$ and $2 \mathrm{mM}$ phosphate buffer using a chip with a working electrode area of $2.4 \mathrm{~mm}^{2}$. Scan rate was $20 \mathrm{mV} / \mathrm{s}$. a) no-flow, b) $0.1 \mu \mathrm{L} / \mathrm{min}$ c) $0.25 \mu \mathrm{L} / \mathrm{min}$.

Without flow, a typical diffusion limited curve can be observed (fig 3.6a). From equation 4 , we can expect all ions directly above the electrode to be converted within a few microseconds. Since a slow scan rate of $20 \mathrm{mV} / \mathrm{s}$ is used, the measured current is due to ions diffusing towards the beginning and end of the working electrode from the rest of the channel.

With increased flow, an added contribution of convection to the measured current is observed. Additionally, the shape of the currents appears to be the sum of the curve without flow and a linear relation depending on applied potential.

In table 1 the peak currents at $-0.4 \mathrm{~V}$ at different flow rates and two chips with different working electrode area are shown. The data listed in the first row of table 1 can 
be fitted using a linear equation $\left(R^{2}=1\right)$ :

$$
I=-0.15 \cdot 10^{-6}-2.2 \cdot Q_{c}
$$

With $Q_{c}$ the volumetric flow velocity in $\mathrm{L} / \mathrm{min}$. Note that the first term is equal to the measured current without flow. If mass-transport is assumed to be the rate limiting step, the measured current is known to be the sum of diffusion and convection. As can be concluded from equation 3.7, the measured current is indeed the sum of a constant diffusion factor and a linear contribution of volumetric flow.

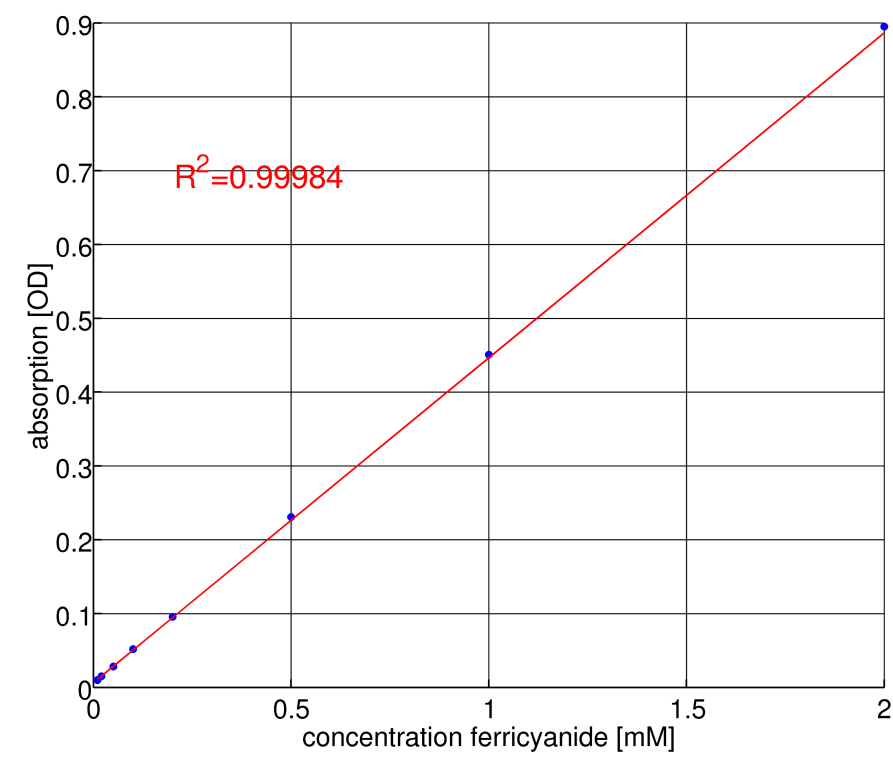

Figure 3.7: Calibration curve for UV/vis absorbance of ferricyanide.

A second observation from table 3.1, is that the current does not depend on the working electrode area as in normal bulk situation. Apparently all ions are converted at a small part of the working electrode. Therefore we assume that all introduced ions are converted.

We also tried to measure the conversion efficiency by integrating the measured current over time, while sufficient overpotential was applied to convert ferrocyanide into ferricyanide. The obtained result thus gives an estimate for the amount of charge transferred during the conversion. At total conversion this amount of charge should be equal to the amount of analyte present in the chip during conversion. However, 
due to small variations in the flow velocity, which was induced by the syringe pump, this method was not accurate enough. At zero flow, this method suffered from lateral diffusion from the channel inlets to the edges of the electrode.

\subsubsection{Conversion efficiency study}

Ferricyanide is known to show UV/vis absorbance at 418nm [29]. Prior to the conversion efficiency measurements, the UV/vis absorbance at $418 \mathrm{~nm}$ is calibrated for different ferricyanide concentrations. The result of this calibration is shown in figure 3.7.

Next, a mixture of $1 \mathrm{mM}$ ferricyanide, $1 \mathrm{mM}$ ferrocyanide, $0.5 \mathrm{M}$ KNO3 and $2 \mathrm{mM}$ phosphate buffer $(\mathrm{pH}=7)$ is oxidized at $0.5 \mathrm{~V}$ or reduced at $-0.5 \mathrm{~V}$ on-chip using a flow rate of $1 \mu \mathrm{L} / \mathrm{min}$. The resulting reaction products are measured optically. The recorded UV/vis absorbance is shown in figure 3.8. At oxidating potentials, ferrocyanide is converted into ferricyanide. For reductive potentials, the reaction is reversed. As a result, the ferricyanide concentration will be decreasing at negative potentials.

As expected, the absorbance is indeed higher at the oxidizing potential of $0.5 \mathrm{~V}$, and lower at the reducing potential of $-0.5 \mathrm{~V}$. From the measurement data, the conversion efficiency is calculated to be $97 \%$. Therefore almost almost total conversion was achieved.

\subsubsection{Amodiaquine experiments}

The oxidative metabolism of amodiaquine (AQ, $m / z 356$ in positive ion mode) has been investigated by Lohmann et al.7 by isolated liver cell microsomes and by an electrochemical conversion in a flow-through cell. Using the results of those approaches the metabolic pathway, as demonstrated in figure 3.9, was developed.

The aim of our study is to generate the main oxidative metabolites, Amodiaquine quinone imine (AQQI, $m / z$ 354), N-dehylated AQQI (DESAQQI, $m / z$ 326), N-dehylated AQ (DESAQ, $m / z$ 328) and AQ aldehyde (AQ-ald., $m / z$ 299) on a chip and in comparison on a commercial wall-jet cell (Flexcell, Antec Leyden).

As expected, the flow rates had to be reduced when using a wall-jet geometry instead of the flow-through cell with its large electrode surface. The optimum flow rate for the chip was determined as $1 \mu \mathrm{L} / \mathrm{min}$. and for the Flexcell as $10 \mu \mathrm{L} / \mathrm{min}$. With both cells an oxidative potential of $1000 \mathrm{mV}$ is applied to the working electrode. 


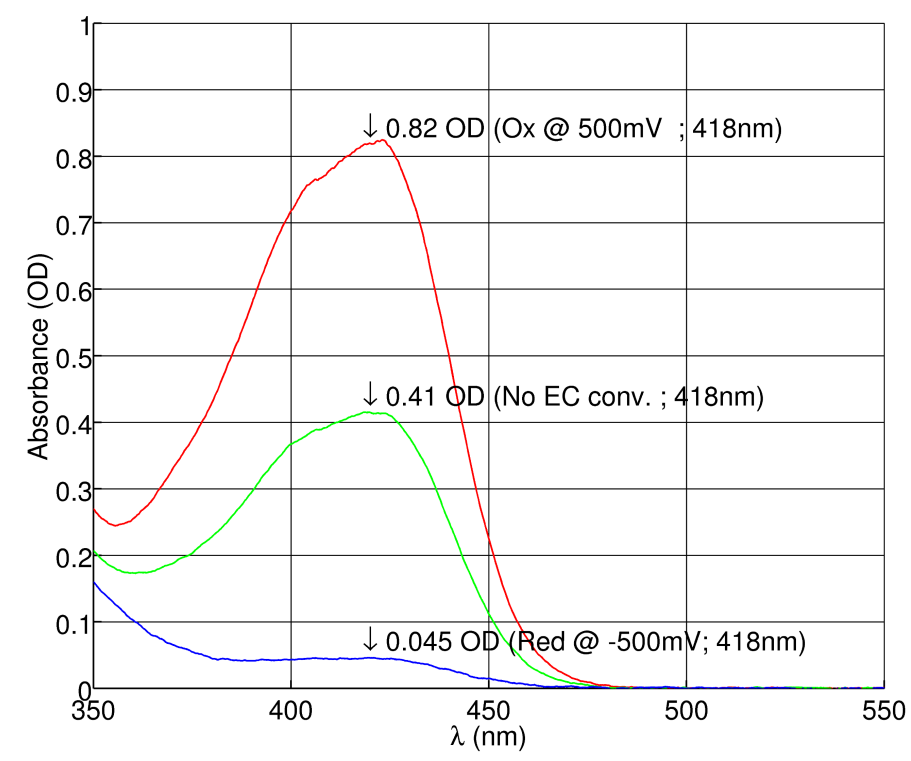

Figure 3.8: UV/vis absorbance of ferricyanide measured in a mixture of $1 \mathrm{mM} / 1 \mathrm{mM}$ ferro-/ferricyanide (before EC conversion) and $0.5 \mathrm{M} \mathrm{KNO} \mathrm{K}_{3}$ supporting electrolyte and $2 m M$ phosphate buffer $(\mathrm{pH}=7)$.

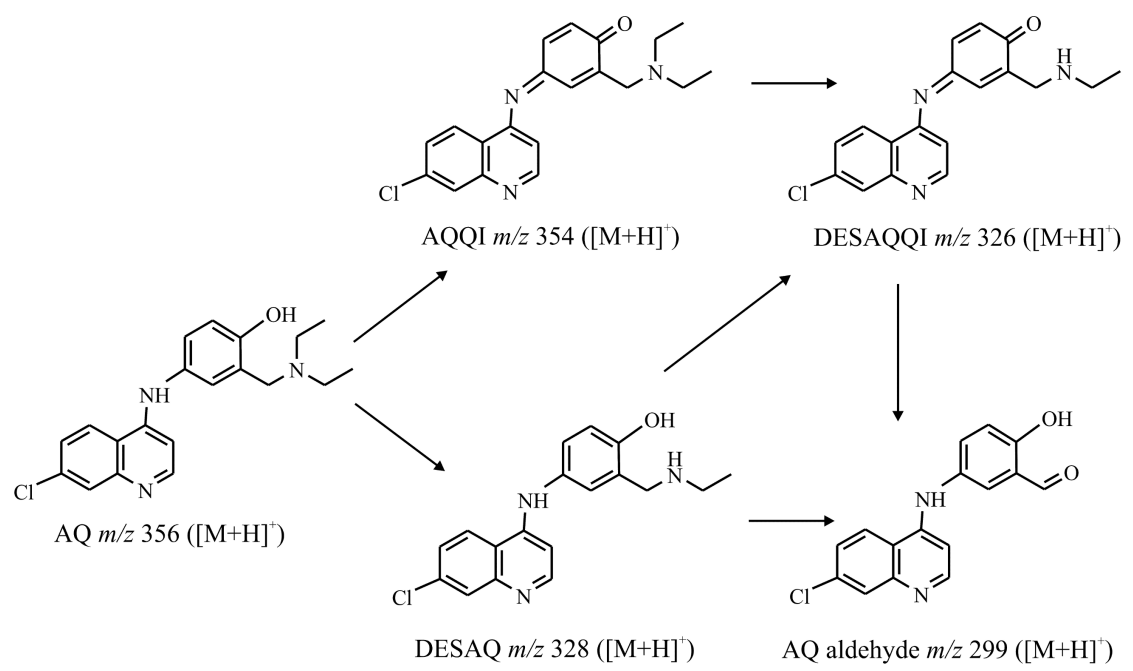

Figure 3.9: Electrochemical metabolism pathway of Amodiaquine. 
The chromatograms of the reaction products of both cell types are shown in figure 3.10. With both cells, the main expected metabolites with $\mathrm{m} / \mathrm{z} 356,328,354,326$ and 299 are observed. Therefore, it can be concluded that the electrochemical cell on chip gives comparable results as the wall-jet cell (Flexcell). Further, using the chip, the conversion of AQ into its metabolites is as high as the conversion in the Flexcell.

However, compared to the EC-UV/vis study, lower conversion efficiency is observed. In equation 6 and the experiments with ferricyanide, mass transport is assumed to be the reaction rate-limiting step. We believe that this is not the case for amodiaquine.

\subsection{Conclusions}

An electrochemical cell on chip for high conversion of the introduced electrochemically active species was developed. The internal volume of the main channel is only 9.6nL. Cyclic voltammograms at various flow rates are recorded on-chip. The measured currents were not influenced by the size of the working electrode. Therefore we believe that all introduced species are converted inside the chip at sufficiently high overpotentials.

Using this chip we were also able to measure the electrochemical conversion of ferricyanide using UV/vis spectroscopy. A conversion efficiency of $97 \%$ has been observed at a flow rate of $1 \mu \mathrm{L} / \mathrm{min}$. Therefore, this chip is able to fully convert small and fast reacting ions.

In the oxidation experiments of amodiaquine, the chip system allows to obtain results similar to those obtained with conventional cell systems at a significantly reduced volume of sample and flow rate. The conversion efficiency considerations presented in the beginning of this chapter and in the experiments with ferricyanide assumed mass transport to be the rate determining step in the total reaction. From our results, we conclude that this is not the case for the oxidation reaction of amodiaquine. However, the chip does show comparable results with the commercial alternatives. 


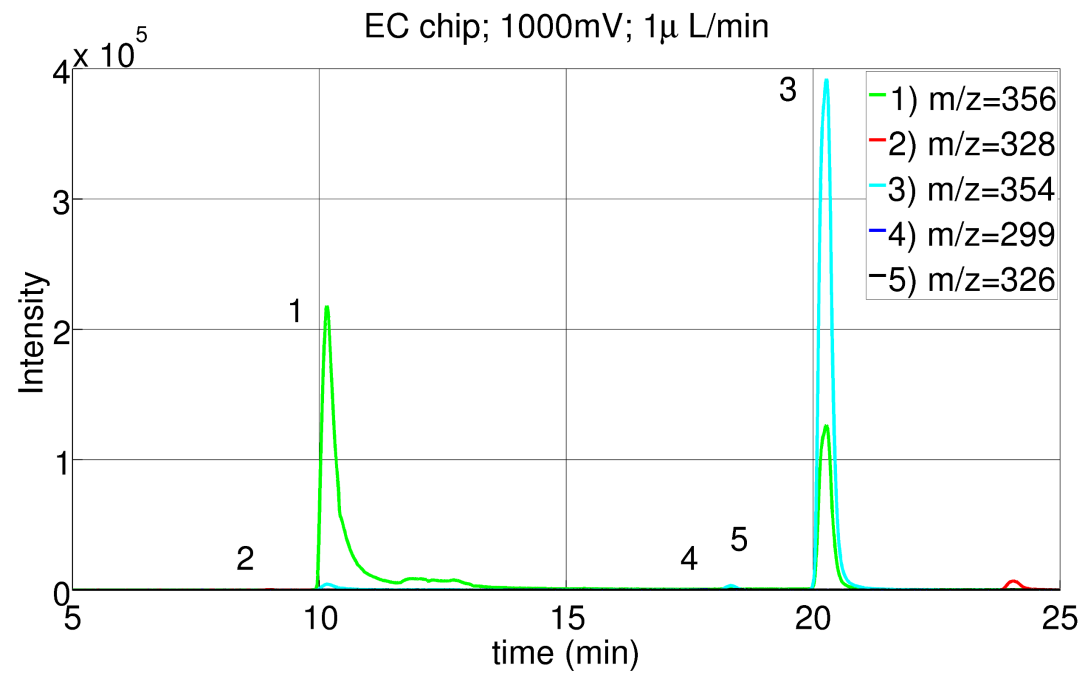

a)

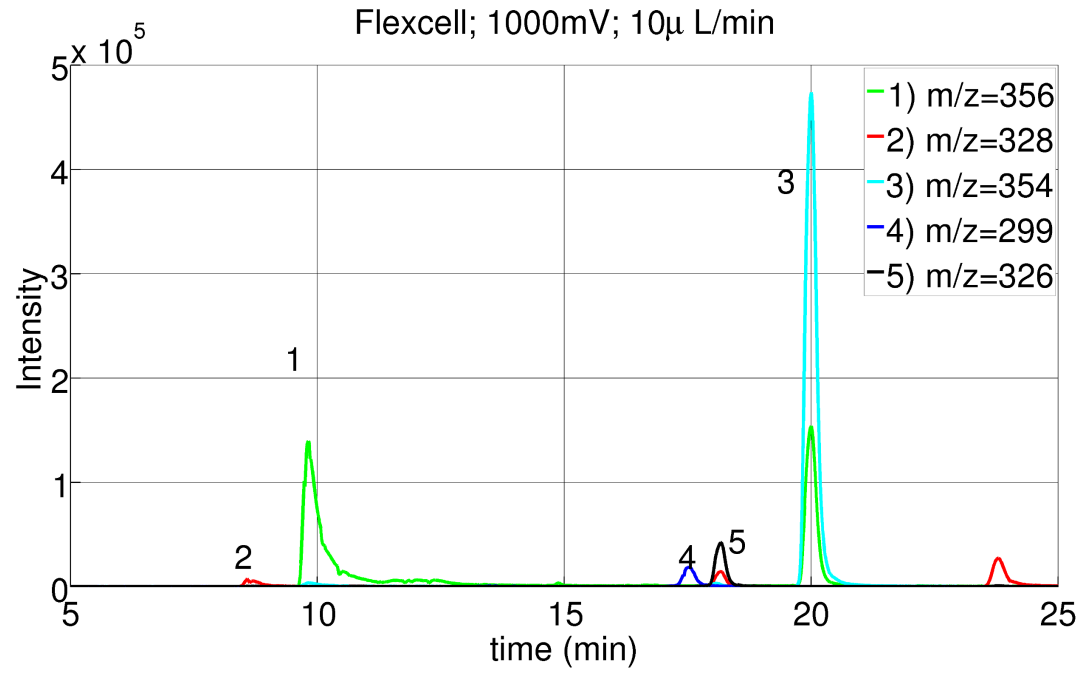

b)

Figure 3.10: Combined extracted ion traces of $A Q(1)$ and the four metabolites $D E$ SAQ (2), AQQI (3), AQ-ald (4) and DESAQQI (5) using our chip (a) and the flexcell from Antec Leyden (b). 


\section{Bibliography}

[1] M. Odijk, A. Baumann, W. Lohmann, F. T. G. van den Brink, W. Olthuis, U. Karst, and A. van den Berg. Lab on a Chip, 9:1687-1693, 2009.

[2] A. P. Bruins. Journal of Chromatography A, 794:345-357, 1998.

[3] G. Diehl and U. Karst. Analytical Bioanalytical Chemistry, 373:390-398, 2002.

[4] H. Hayen and U. Karst. Anal Chem, 75:4833-4840, 2003.

[5] U. Karst. Angewandte Chemie Int Ed, 43:2476-2478, 2004.

[6] F. Zhou and G. van Berkel. Anal Chem, 67:3643-3649, 1995.

[7] W. Lohmann and U. Karst. Analytical Bioanalytical Chemistry, 391:79, 2008.

[8] U. Jurva, H. V. Wikstrom, and A. P. Bruins. Rap Comm in Mass Spectrometry, 14:529-533, 2000.

[9] K. J. Volk, R. A. Yost, and A. Brajter-Toth. Anal Chem, 61(15):1709-1717, 1989. ISSN 0003-2700.

[10] S. van Leeuwen, B. Blankert, J. Kauffmann, and U. Karst. Analytical Bioanalytical Chemistry, 382:742-750, 2005.

[11] W. Lohmann and U. Karst. Anal Chem, 79:6831-6839, 2007.

[12] H. Permentier, U. Jurva, B. Barroso, and A. Bruins. Rap Comm in Mass Spectrometry, 17(14):1585-1592, 2003.

[13] K. J. Volk, M. S. Lee, R. A. Yost, and A. Brajter-Toth. Anal Chem, 60:720-722, 1988.

[14] B. H. Westerink. Journal of Chromatography B, 747:21-32, 2000.

[15] H. Deng and G. V. Berkel. Anal Chem, 71:4284-4293, 1999. doi:10.1021/ ac990527y.

[16] M. C. S. Regino and A. Brajter-Toth. Anal Chem, 69:5067-5072, 1997. doi: 10.1021/ac961261n.

[17] G. R. Bruckenstein S. J Am Chem Soc, 93:793-794, 1971.

[18] G. Hambitzer and J. Heitbaum. Anal Chem, 58(6):1067-1070, 1986.

[19] X. Xu, W. Lu, and R. B. Cole. Anal Chem, 68:4244-4253, 1996. doi:10.1021/ ac960362i.

[20] W. Lu, X. Xu, and R. Cole. Anal Chem, 69:2478-2484, 1997. doi:10.1021/ ac9612842.

[21] B. H. Timmer, K. M. van Delft, R. P. Otjes, W. Olthuis, and A. van den Berg. Analytica Chimica Acta, 507:137-143, 2004.

[22] S. Bohm, D. Pijanowska, W. Olthuis, and P. Bergveld. Biosensors and Bioelectronics, 16:391-397, 2001.

[23] M. Odijk, W. Olthuis, V. Dam, and A. van den Berg. Electroanalysis, 20(5):463-468, 2008. 
[24] V. A. T. Dam, W. Olthuis, and A. van den Berg. The Analyst, 1:1, 2007. doi: 10.1039/b616667a. DOI: 10.1039/b616667a.

[25] W. de Malsche, H. Eghbali, D. Clicq, J. Vangelooven, H. Gardeniers, and G. Desmet. Anal Chem, 79(15):5915-5926, 2007.

[26] E. Oosterbroek. Ph.D. thesis, University of Twente, 1999.

[27] A. J. Bard and L. R. Faulkner. Electrochemical methods - fundamentals and applications. Wiley, 2nd edition, 2001.

[28] M. Brivio, N. R. Tas, M. H. Goedbloed, H. J. G. E. Gardeniers, W. Verboom, A. van den Berg, and D. N. Reinhoudt. Lab on a Chip, 5:378-381, 2005.

[29] H. Thompson. Advances in spectroscopy, volume 2. Interscience publishers network inc., London, 1961. 


\section{Electrochemistry-on-chip for use in drug metabolism studies}

We have designed an integrated 3-electrode electrochemical cell (EC) on-chip with high analyte conversion rates for use in drug metabolism studies. The EC contains platinum working and counter electrodes and an iridium oxide pseudo-reference electrode. The pseudo-reference electrode has a $\mathrm{pH}$ sensitivity of $-52 \mathrm{mV} / \mathrm{s}$, and thus will provide a constant potential in solutions with known and constant $\mathrm{pH}$. The average drift of the iridium oxide electrode is below $5 \mathrm{mV}$ for a typical 15 minutes conversion experiment. We have been able to mimic the oxidative drug metabolism reactions catalysed by enzymes of the cytochrome P-450 family, normally occurring in the human body. With the chip, the different reaction products of both rat liver cell microsome and human liver cell microsome incubations have been observed. ${ }^{1}$

\footnotetext{
${ }^{1}$ Modified from: a paper published Biosensors and Bioelectronics [1]. The original paper is written in close cooperation with the Institute of Inorganic and Analytical Chemistry of the University of Münster. Co-authors are A. Baumann, U. Karst, W. Olthuis and A. van den Berg.
} 


\subsection{Introduction}

Electrochemistry (EC) is recently gaining more attention as a tool in rapid, online drug screening as it can be used to mimic oxidation reactions occurring in the human body. Oxidation reactions play a crucial role in the biotransformation of drugs. By introducing polar functional groups, like hydroxyl groups, into the drug molecule, the organism facilitates the detoxification and excretion of xenobiotics [2]. However, from the biotransformation of specific compounds, metabolites may arise, which bear an increased toxicity (bioactivation). One typical example are quinones and quinone imines, which are very often highly reactive, electrophilic compounds, formed upon oxidative metabolism [3]. During drug development processes, drug candidates, potentially undergoing bioactivation into toxic metabolites, have to be excluded from the further development process as early as possible. Conventional drug metabolism studies include in vitro tests, utilizing liver cells and liver cell extracts [4] as well as in vivo tests with laboratory animals. Most frequently used cell extracts to study oxidative metabolism reactions are liver microsomes from humans and rats (HLM and RLM). Liver microsomes contain a high enzyme concentration of the cytochrome P-450 group, which catalyzes the majority of oxidative metabolism reaction [5].

As a purely instrumental alternative to investigate drug oxidation reactions, the electrochemical simulation has been developed. Potential oxidative metabolites are generated in an electrochemical cell and are subsequently identified by on-line liquid chromatography/mass spectrometry (EC/LC/MS). The major benefit of this instrumental approach, compared to metabolism studies based on hepatic cell matrices, is that reactive metabolites can be directly detected. In vitro, those reactive metabolites frequently remain unidentified, because the compounds bind to proteins in the cell matrix [6]. Moreover, besides being a rapid screening tool for oxidative metabolites, EC/LC/MS can be used to study the reactivity of metabolites towards cellular compounds like proteins or DNA and thus allows the evaluation of a potential liver toxicity [7].

Examples of the electrochemistry-based drug screening in comparison to traditional techniques are described in literature $[8,9]$ where the metabolic pathway of tetrazepam and clozapine is studied. Tetrazepam is a benzodiazepine derivative and mainly used as muscle relaxant, while clozapine is an anti-psychotic drug. Also reported is the electrochemical oxidation of metoprolol [10], a selective $\beta 1$ receptor blocker. In that paper, it is described to which extent electrochemical oxidation can mimic typical metabolism reactions. Current developments and applications of the electrochemical metabolism simulation have been summarized in three reviews [11-13]. 
Recently, a paper has been published where electrochemical oxidation of a drug is studied to mimic the phase-1 metabolites of Verapamil, a known antiarrythmic drug [14]. The chip fabricated in this study was using the photo-catalytic effect of titanium dioxide to oxidise the introduced species. This chip also contained an electrospray ionisation needle to directly introduce the metabolites into the MS, thus minimizing the volume between the reaction area and the ionisation interface.

We have already shown that a microfluidic electrochemical cell can be used successfully [15] to study e.g. the metabolic pathway of amodiaquine, a known antimalarial agent. The major benefits of using a microscale electrochemical cell is that diffusion lengths are greatly reduced. Therefore, reaction rates are no longer determined by diffusive mass transport and we have observed total conversion of electrochemically active species for small and fast reacting ions. Moreover, the required sample volumes are reduced, since the internal volume of the chip is typically in the nanoliter range. The major advantage of our chip over the photo-catalytic chip [14] is that we have more control over the electrochemical reactions taking place. Moreover, we can also measure the oxidation current. Finally, we can achieve higher oxidation potentials than possible with the photo-catalytic chip. Therefore, a wider range of oxidation reactions mimicked by the electrochemical cell can be observed, which is especially important in (partly) organic solutions were the conductivity is lower.

Here we present an improved version of the electrochemical 3-electrode cell on-chip. The previous chip [15] contained a palladium pseudo-reference electrode. Using this metal, the fabrication process is limited to temperatures up to $500^{\circ} \mathrm{C}$. At temperatures above $500^{\circ} \mathrm{C}$ the palladium thin-film starts to agglomerate. Therefore, the bonding of glass during the chip fabrication was not as good as desired. As a result, the previous chip could not withstand fluidic pressures above approximately $500 \mathrm{kPa}$. The mechanical stability of the palladium film was inferior if the chip was used for longer periods of time. Especially in strong acidic solutions the film detached after a few minutes, probably caused by intrinsic stress induced by hydrogen uptake into the palladium [16]. In the improved version of this chip, the palladium electrode is replaced by an iridium oxide ( $\mathrm{IrOx}$ ) film. IrOx is mainly known as $\mathrm{pH}$ electrode [17-19]. However, in solutions with known $\mathrm{pH}$ it can be used as pseudo-reference electrode [20-22]. The major benefit of using IrOx is that it can withstand much higher temperatures [23], which is especially important for chip fabrication.

We have tested both the mechanical and electrochemical stability of the IrOx pseudoreference electrode, of which the results are included in this chapter. Moreover, the metabolism pathway of procainamide is studied on-chip. Procainamide is a known antiarrythmic drug [24]. In this chapter mass spectrometry (MS) measurements of 
the different metabolites separated by liquid chromatography (LC) are reported. We compare MS results from liver cell microsome incubations to MS results from electrochemical oxidation on-chip, to show that the chip is a feasible alternative to microsomal studies.

\subsection{Materials and methods}

\subsubsection{Chip fabrication}

The chip design is aimed at high conversion of introduced analyte. In chapter 3 , it is explained how the geometry of electrodes and fluidic channels is optimized towards high-turnover rates [15]. The chip layout presented here is designed following the same design principles as described earlier.

In figure 4.1a, a photo of a finished chip is shown. The fluidic port at the right side is used as main inlet. The fluidic port at the left side is connected to the external LC and MS devices. The fluidic port on the lower-left side is used as outlet during measurements, to extract a fraction of the total injected sample volume. Only during cleaning, this port is used as a second inlet. The platinum working electrode is located in the main fluidic channel. This channel is $4 \mu \mathrm{m}$ high, $400 \mu \mathrm{m}$ wide and $10 \mathrm{~mm}$ long. On the right side of the working electrode, the IrOx reference electrode is visible. The platinum counter electrode is placed in a separate side-channel to prevent unwanted species to appear in the measured mass spectra. This channel is much larger to contain the larger counter electrode. The counter electrode is made this large to prevent gas formation inside the chip. The main channel volume is only $16 \mathrm{~nL}$, while the total chip volume is $180 \mathrm{~nL}$.

The chip is made of two glass borofloat wafers. On the first wafer, wells are etched using buffered HF to adjust the electrode surface $10 \mathrm{~nm}$ below the glass surface. Next, electrodes are patterned by lithography using lift-off techniques. A thin-film of $135 \mathrm{~nm}$ platinum is deposited onto an adhesion layer of $10 \mathrm{~nm}$ tantalum. For the IrOx, the same etching and lift-off steps are repeated. A thin film of $73 \mathrm{~nm}$ IrOx is sputtered on a sandwich of $10 \mathrm{~nm}$ titanium, $107 \mathrm{~nm}$ platinum and $10 \mathrm{~nm}$ titanium, where titanium is acting as adhesion layer and platinum as conducting layer. The iridium oxide is sputtered using an iridium 4 inch target (obtained from Kurt J. Lesker). The sputtering power applied was $46 \mathrm{~W}$ DC, with $20 \mathrm{sccm}$ Argon and $20 \mathrm{sccm}$ oxygen gas flow into the sputtering chamber. This sputtering is conducted using an in-house developed sputtering machine, making it possible to keep the wafer in vacuum for the deposition of the whole $\mathrm{IrOx} / \mathrm{Ti} / \mathrm{Pt} / \mathrm{Ti}$ sandwich layer. 

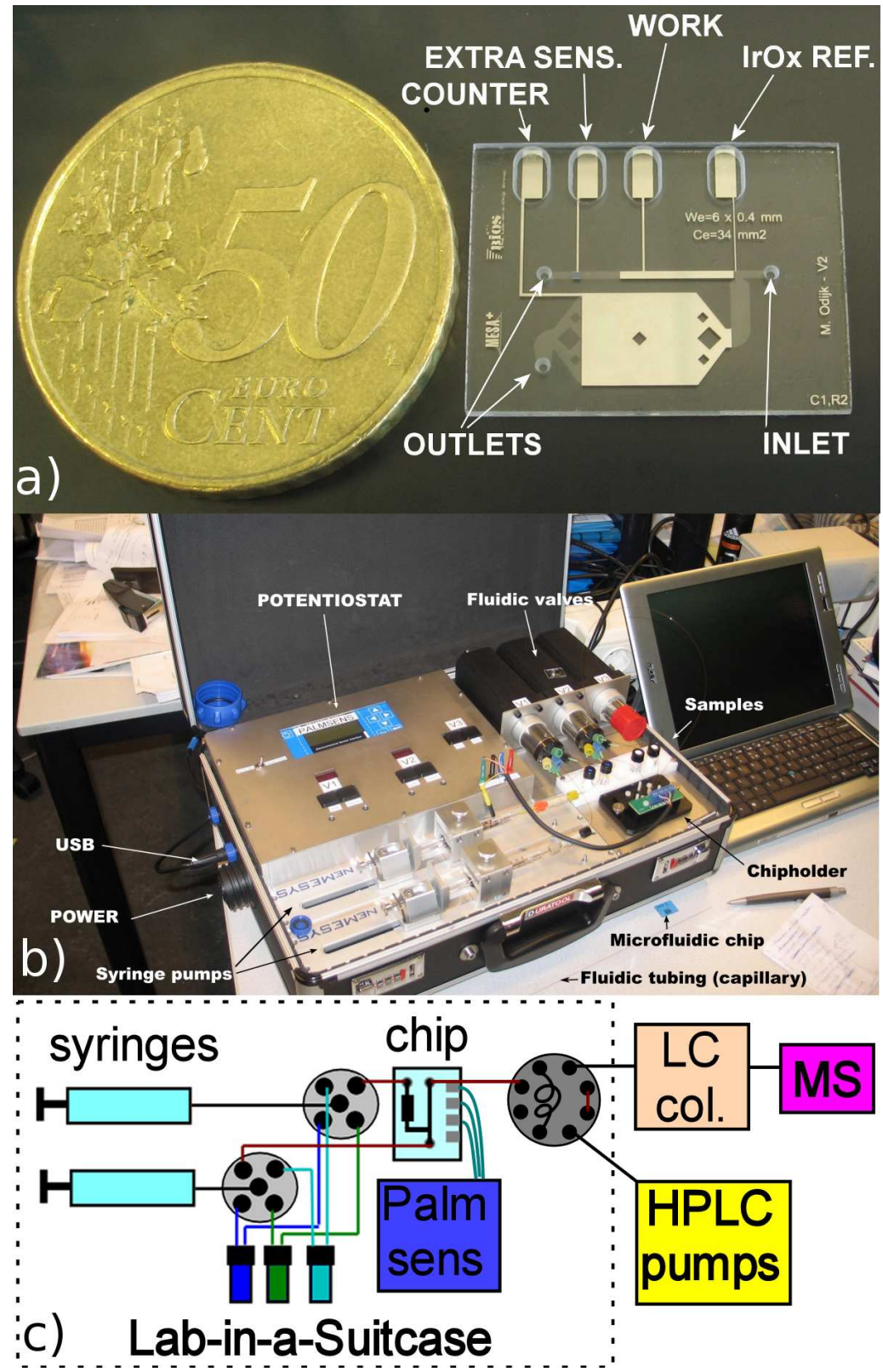

Figure 4.1: (a) Photo of the electrochemical cell on-chip. In the picture, fluidic inlet and outlets and the working, reference and counter electrode are indicated. (b) Photo of the Lab-in-a-Briefcase setup. In the picture, chipholder, syringe pumps, fluidic switching and injection valves, sample vials and the portable potentiostat are indicated. (c) Schematic overview of the total setup including the Lab-in-a-Briefcase, LC pumps and mass spectrometer. 
On the second wafer, a layer of $10 \mathrm{~nm}$ chromium and $100 \mathrm{~nm}$ gold is sputtered, which acts as etching mask for the fluidic channels. These $4 \mu \mathrm{m}$ deep channels are etched into the glass with $25 \% \mathrm{HF}$. Afterwards, Ordyl BF410 foil is laminated onto the other side of the top wafer and patterned with lithography. Fluidic inlets and contact holes for the electrode pads are made using powderblasting. Finally, the foil and the chromium/gold layer is removed and the two wafers are directly bonded at a temperature of $600^{\circ} \mathrm{C}$. In a last step, the combined wafers are diced into individual chips of $15 \times 20 \mathrm{~mm}$. More details on the chip fabrication and the sputtering of iridium oxide can be found in the appendix of this thesis.

\subsubsection{Chemicals}

For the $\mathrm{pH}$-response measurement a linear $\mathrm{pH}$ buffer was used containing $0.033 \mathrm{M}$ boric acid, $0.033 \mathrm{M}$ citric acid, $0.033 \mathrm{M}$ potassium dihydrogenphosphate. This buffer was titrated during the experiment with $0.1 \mathrm{M}$ potassium hydroxide. For the opencircuit measurements, a $0.1 \mathrm{M}$ potassium dihydrogenphospate solution was used with $\mathrm{pH}$ adjusted to 7.4 .

For the electrochemical metabolism study, $100 \mu \mathrm{M}$ procainamide was dissolved in a solution containing $1 \mathrm{mM}$ ammonium acetate. Between each experiment, the chip was flushed with a $50 \%$ acetonitrile / $50 \%$ water solution containing $0.1 \mathrm{v} \%$ acetic acid.

Chemicals used for the microsomal incubation: $50 \mathrm{mM}$ phosphate buffer solution (potassium dihydrogenphosphate, dipotassium hydrogenphosphate, adjusted to $\mathrm{pH}$ 7.4), magnesium chloride hexahydrate and NADPH (AppliChem, Darmstadt, Germany). Pooled male rat liver microsomes (RLM) (Sprague Dawley) and pooled human liver microsomes (HLM) both with a protein concentration of $20 \mathrm{mg} / \mathrm{mL}$, were delivered by BD Bioscience (Woburn, MA, USA). Unless otherwise specified, all chemicals were obtained from Sigma-Aldrich.

\subsubsection{Setup}

The drug metabolism measurements were conducted with the in-house developed Lab-in-a-Briefcase. A photo of this setup is shown in figure 4.1b. An overview of this total setup is given in figure $4.1 \mathrm{c}$.

In the lower right side of figure $4.1 \mathrm{~b}$, the chip is placed inside an in-house developed chipholder. In this holder, fluidic connections are provided by commercially available connectors (nanoports, Upchurch scientific), while electrical connections are provided by spring metal contact probes (ISI Interconnect Specialities Inc.). The 
flows over the chip are regulated in such a way that the generated products at either the working or counter electrode cannot reach the iridium oxide pseudo-reference electrode. This is done using an inward flow of $0.25 \mu \mathrm{L} / \mathrm{min}$ at the inlet port (visible on the right hand side of figure 4.1a) and a syringe pump connected to the sidechannel pumping at an outward flow of $0.125 \mu \mathrm{L} / \mathrm{min}$, which is exactly half of the rate of the inward flow. As a result, the flow rate over the main channel and into the MS is equal to $0.125 \mu \mathrm{L} / \mathrm{min}$. Since, at these flow rates, mass transport due to convection is more dominant compared to mass transport by diffusion, generated products cannot reach and disturb the potential of the pseudo reference electrode. These flow rates have been tested and determined to give the most oxidation products.

The chipholder is connected to two switching valves and one injection valve using fused silica glass capillary (Valco type C75X-6694EMH and C72MX-4698ED, Vici Valco Instruments Co. Inc.). The switching valves are connected to several sample vials and to two syringe pumps (Nemesys, Cetoni GmbH, Germany). The total volume of all the tubing, valves and connectors is calculated to be approximately $5 \mu \mathrm{L}$, excluding two $50 \mu \mathrm{L}$ syringes (Type $1705 \mathrm{~N}$, Hamilton Bonaduz AG).

Potentials are applied to the chip using a portable potentiostat (Palmsens, Palm Instruments BV, the Netherlands). The whole briefcase is controlled by a laptop running a single home-build labview program (Labview, National Instruments Corporation).

The injection valve was connected to a sample loop of $5 \mu \mathrm{L}$. After filling the loop with the analyte, the valve was switched and a sample plug was flushed with $500 \mu \mathrm{L} / \mathrm{min}$ onto a LC column. The LC separation was performed on a Nucleodur Sphinx RP column (Macherey-Nagel, Düren, Germany) with the dimension 150x3 mm, particle size $5 \mu \mathrm{m}$ and pore size of $10 \mathrm{~nm}$. The mobile phase consists of $10 \mathrm{mM}$ aqueous ammonium formate buffer with $0.02 \%$ formic acid as eluent $\mathrm{A}$ and acetonitrile as eluent B. For the separation, the following gradient programme was used: 0-2 min isocratic at $15 \% \mathrm{~B}, 2-10$ min gradient from 10 to $50 \% \mathrm{~B}, 10-11 \mathrm{~min}$ gradient from $50-95 \% \mathrm{~B}, 11-13 \mathrm{~min}$ isocratic at $95 \% \mathrm{~B}, 13-16$ min equilibration at $15 \% \mathrm{~B}$.

A LC system was used from Shimadzu (Duisburg, Germany) coupled to a QTRAP mass spectrometer with an electrospray ionization (ESI) source (Applied Biosystems, Darmstadt, Germany), working in positive ion mode. The LC system consists of two LC pumps (LC-10ADVP), a degasser (DGU-14A), an autosampler (SILHTA), a column oven (CTO-10AVP) and an UV-detector (SPD-10AVVP) operating at $254 \mathrm{~nm}$. Control of the LC/MS system and data handling was carried out by the software Analyst 1.4.1 (Applied Biosystems). For the determination of exact masses and molecular formulas, single measurements were repeated using an LC system 
from Antec Leyden (Zoeterwoude, The Netherlands) coupled to a microTOF mass spectrometer, which was equipped with an ESI source (Bruker Daltonics, Bremen).

The $\mathrm{pH}$ response and potential stability measurements of the IrOx pseudo-reference electrode were conducted off-chip, using a processed wafer which is not bonded to the wafer containing the fluidic channels and inlets. These measurements are recorded with a VSP potentiostat (Bio-Logic SAS, France) using a silver/silverchloride saturated $\mathrm{KCl}$ reference electrode (Radiometer Analytical) and platinum counter electrode. The $\mathrm{pH}$ was measured using a battery-powered portable $\mathrm{pH}$ meter $(\mathrm{Cy}-$ berScan $\mathrm{pH} 310$, Eutech Instruments). During the determination of the $\mathrm{pH}$, the $\mathrm{IrOx}$ electrode was removed temporarily from the solution to prevent interference with the $\mathrm{pH}$ meter.

\subsubsection{Methods}

\subsubsection{Electrochemical metabolism simulation}

For the on-chip drug metabolite study, a series of experiments was conducted to determine the optimal potential. The optimal potential for oxidation was determined to be $1.75 \mathrm{~V}$, since it gave the most oxidation products without generating compliance errors on the potentiostat. This potential was applied for 60 minutes before injecting the sample plug onto the LC column, to make sure that the sample loop was completely filled with the generated oxidation products.

\subsubsection{Microsomal incubations}

A mixture of microsomal protein and procainamide, dissolved in phosphate buffer solution was preincubated for $5 \mathrm{~min}$ at $37^{\circ} \mathrm{C}$. Magnesium chloride and NADPH were added to the incubation mixture, which was then further incubated at $37^{\circ} \mathrm{C}$ for 90 minutes. The total volume of the incubation mixture was $500 \mu \mathrm{L}$ for RLM incubation mixtures and $250 \mu \mathrm{L}$ for HLM incubation mixtures. The final concentrations were as follows: $1.3 \mathrm{mg} / \mathrm{mL}$ microsomal protein, $50 \mu \mathrm{M}$ procainamide, $0.5 \mathrm{mM}$ magnesium chloride, $1.2 \mathrm{mM}$ NADPH. Subsequent to the incubation, proteins were precipitated by adding $500 \mu \mathrm{L}$ acetonitrile $(250 \mu \mathrm{L}$ for HLM). After centrifugation, the supernatant was analysed by LC/MS. The LC/MS conditions are the same as described for the electrochemical simulation, only the injection volume was increased to $10 \mu \mathrm{L}$. As negative control, a second incubation without NADPH was performed. 


\subsection{Results and discussion}

\subsubsection{IrOx $\mathrm{pH}$ response}

In figure 4.2, the potential of the $\mathrm{IrOx}$ electrode is shown in the $\mathrm{pH}$ range of 2.7 to 5.5, measured versus an $\mathrm{Ag} / \mathrm{AgCl}$ sat. $\mathrm{KCl}$ reference electrode. The measured data points are fitted to a linear response of $-52 \mathrm{mV} / \mathrm{pH}$, which is slightly lower than the theoretically expected value of $-59 \mathrm{mV} / \mathrm{pH}$ [25]. The $\mathrm{pH}$ response strongly depends on the way it is fabricated as shown in literature in a nice summary [19]. In this summary the $\mathrm{pH}$ response for sputtered IrOx films (SIROFs) varies between -54 to $-60 \mathrm{mV}$ at room temperature, which is slightly higher than our measured value.

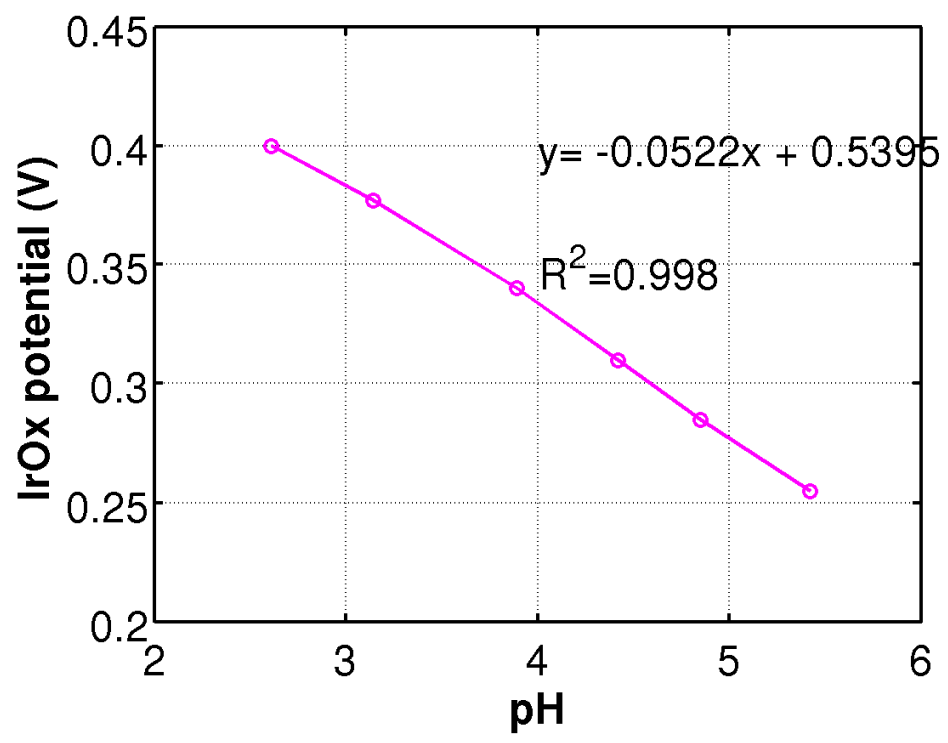

Figure 4.2: $\mathrm{pH}$ sensitivity of the IrOx electrode. Potential measured vs. Ag/AgCl sat. $\mathrm{KCl}$ reference electrode. The IrOx electrode shows a $\mathrm{pH}$ sensitivity of $-52 \mathrm{mV} / \mathrm{pH}$.

\subsubsection{IrOx stability}

The potential of the IrOx is also observed for a period of 29 hours in a phosphate buffer solution with $\mathrm{pH}$ adjusted to $\mathrm{pH}$ 7.4. The results are shown in figure 4.3. This experiment was carried out at ambient temperature. Also, the solution was exposed to air to get a similar environment to the drug metabolism studies. Exposure to air can possibly influence the potential of the IrOx electrode, because of interaction with dissolved oxygen in the solution [26]. 
The initial potential shift of the IrOx electrode was $50 \mathrm{mV}$ over a period of 15 hours after the start of the experiment, followed by a long-term drift within a range of $8 \mathrm{mV}$. The initial drift is known to result from hydration effects after the initial film fabrication $[19,27]$. The maximum drift was approximately $10 \mathrm{mV} /$ hour during the initial phase. The long-term drift was below $2 \mathrm{mV} /$ hour.

In other literature describing IrOx pseudo-reference stability measurements [22], an initial drift of $120 \mathrm{mV}$ over 1 day and a long-term drift less than $20 \mathrm{mV}$ over 9 days is reported using anodically grown IrOx films (AIROFs). Compared to the drift values reported in literature, our IrOx electrode performs better, both in terms of initial and long-term drift.

Compared to the large overpotentials applied during drug metabolite studies, this drift is well within acceptable limits. A typical drug conversion experiment takes approximately 15 to 20 minutes. Therefore, the expected drift of the pseudo-reference is estimated to be below $5 \mathrm{mV}$ for a single experiment.

Over a period of 8 months of continuous exposure of $\mathrm{IrOx}$ to both neutral and acidic solutions we have not observed any detachment or mechanical deformation, thus indicating the excellent long term stability of the electrodes.

\subsubsection{Procainamide metabolism study}

Procainamide (p-amino-N-[2-(diethylamino)ethyl]benzamide, PA, $m / z$ 236), is given for the treatment of both ventricular and supra-ventricular arrhythmias. It is especially useful in the case of lidocaine-resistant ventricular arrhythmias during acute myocardial infarction [24]. The metabolism mechanism is indicated in figure 4.4. During biotransformation of procainamide, conjugation reactions take place, which result in the formation of $\mathrm{N}$-acetylprocainamide. Upon oxidative metabolism $\mathrm{N}$ monodealkylprocainamide (DEPA, $m / z$ 208), $\mathrm{N}$-hydroxylamine procaineamide (NOH$\mathrm{PA}, \mathrm{m} / z, 252$ ) and the N-oxide derivative of procainamide (NO-PA, $m / z, 250$ ) are formed as major metabolites. The latter metabolite is highly reactive and may bind to macromolecules, leading to the drug-induced lupus erythematosus syndrome observed during chronic therapy with procainamide [24]. The goal of our study is to simulate the formation of the main oxidative metabolites of PA with the on-chip electrochemical cell and to compare it with the results of the conventional microsomal studies.

Fundamentally, there are differences between direct electrochemical and enzymatic oxidation as the underlying mechanisms are different. However, a large number of reactions can be simulated by EC/MS, while only a few of these reactions cannot 


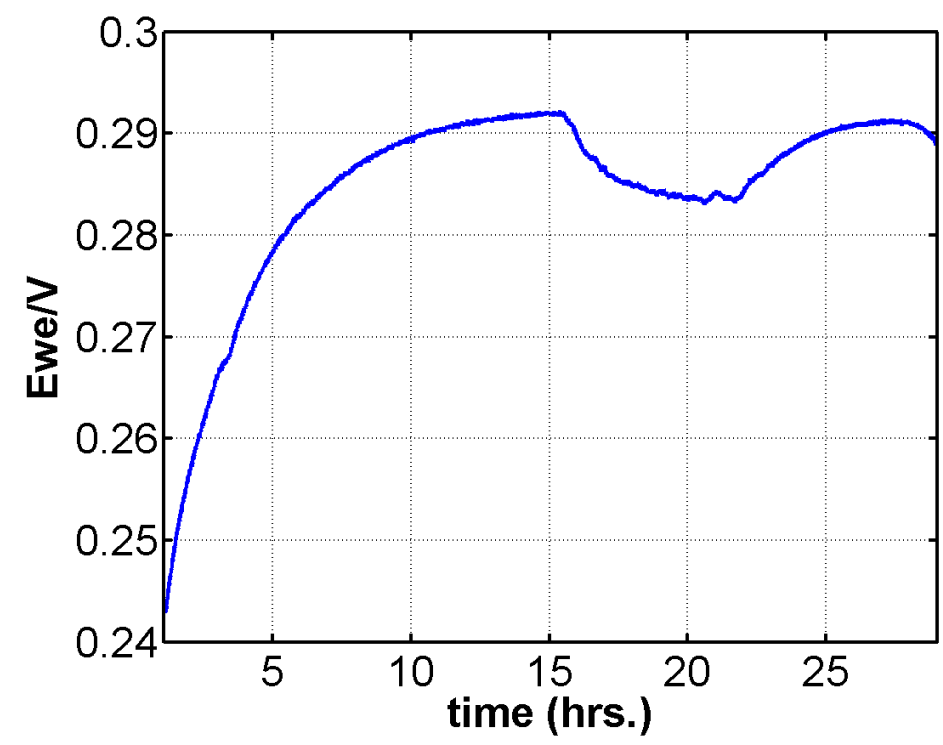

Figure 4.3: Potential stability measurements of the IrOx electrode. Potential measured vs. $\mathrm{Ag} / \mathrm{AgCl}$ sat. $\mathrm{KCl}$ reference electrode. An initial drift of $50 \mathrm{mV}$ and a long-term drift within a range of $8 \mathrm{mV}$ is observed.

be simulated as described in several review articles [11,12]. The most precise data are generated from isolated enzymes of the CYP450 family. However in the organism, there is a strong variation in kind and concentration of the individual enzymes of this group. Therefore, data obtained using individual enzymes are scientifically well defined, but not always the best way of predicting toxic effects on a complex organism. EC/MS certainly will never replace the use of CYP450 enzymes (isolated or in microsomes), but it can be used as complementary method.

A series of experiments was conducted to determine the optimal flow rate for use with the chip. The result of this study is shown in figure 4.5. In the figure, the value of the peak area for the main compound procainamide ( $\mathrm{m} / \mathrm{z} 236)$ was devided by a factor 10 for clarification purposes. For practical, fast conversion experiments a flow rate of $0.5 \mu \mathrm{L} / \mathrm{min}$ over the main channel already gives enough oxidation products for the MS measurements. A flow of $0.5 \mu \mathrm{L} / \mathrm{min}$ is also the most practical value, since the resulting required time to fill the sample loop is 15 minutes which is similar to the time required for the separation in the LC column. In rest of this chapter, a flow rate of $0.125 \mu \mathrm{L} / \mathrm{min}$ over the main channel was used, since it generates the most oxidation products.

In-vitro microsomal incubations have been conducted with rat liver microsomes (RLM) 


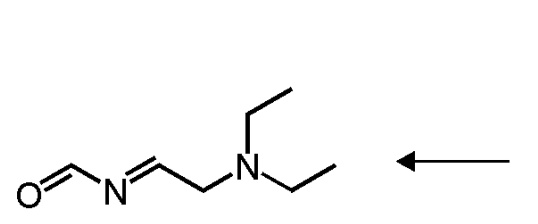

Oxidation product $[\mathrm{M}+\mathrm{H}]^{+} 143$<smiles>CCN(CC)CCNC(=O)c1ccc(NO)cc1</smiles>

Oxygenation product $[\mathrm{M}+\mathrm{H}]^{+} 252$

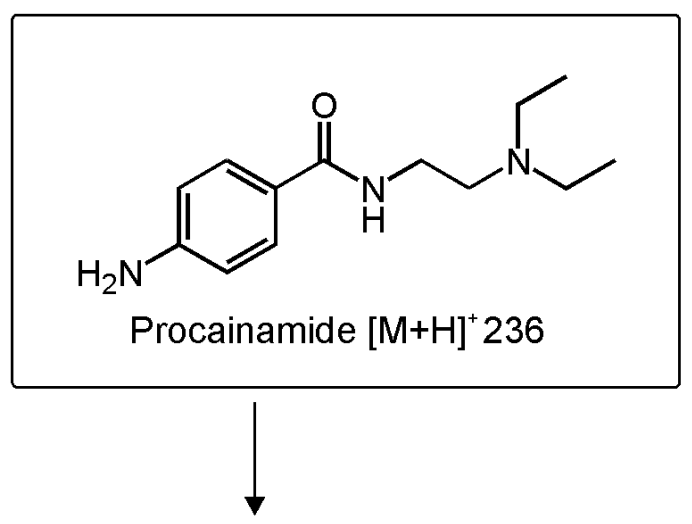<smiles>CCN(CC)CCNC(=O)c1ccc(N=O)cc1</smiles>

Dehydrogenation product $[\mathrm{M}+\mathrm{H}]^{+} 250$

Figure 4.4: Oxidative metabolites of procainamide $(\mathrm{m} / \mathrm{z}, 236)$. Upon oxidative metabolism N-monodealkylprocainamide (DEPA, $\mathrm{m} / \mathrm{z} 208$ ), $\mathrm{N}$-hydroxylamine procaineamide (NOH-PA, $\mathrm{m} / \mathrm{z} 252$ ) and the $\mathrm{N}$-oxide derivative of procainamide (NO-PA, $\mathrm{m} / \mathrm{z} 250)$ are formed as major metabolites. 


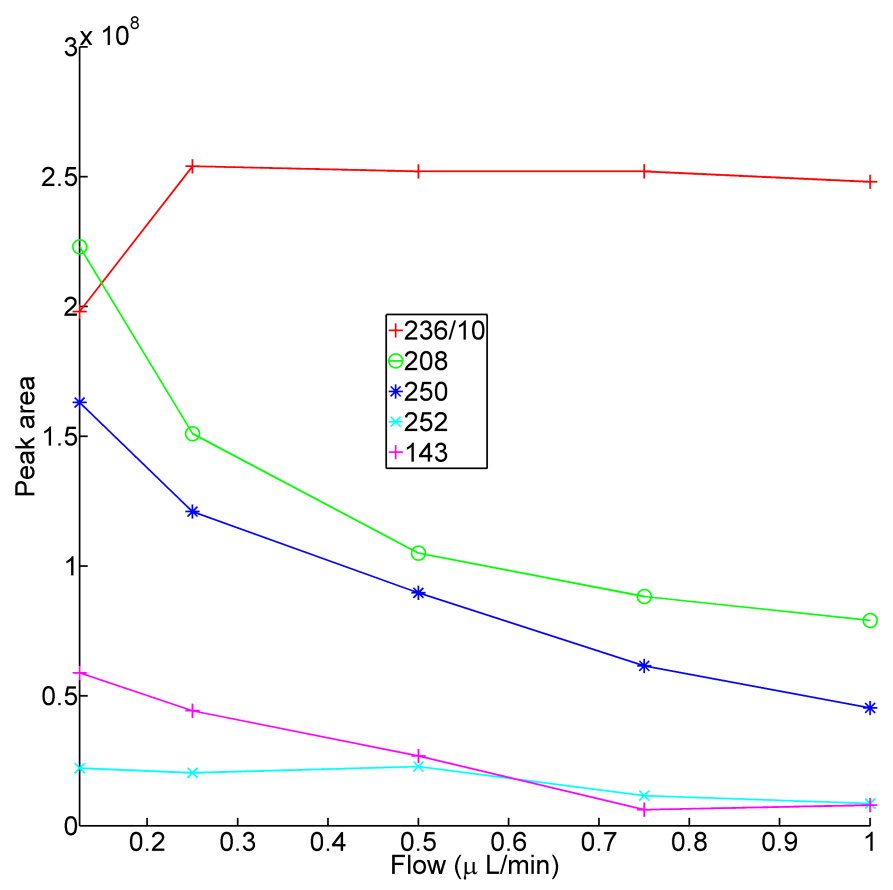

Figure 4.5: Peak areas of procainamide and its main metabolites at various flow rates over the main channel of the chip. The legend indicates the mass traces of $P A$ $(\mathrm{m} / \mathrm{z} 236), D E P A(\mathrm{~m} / \mathrm{z} 208), \mathrm{NO}-\mathrm{PA}(\mathrm{m} / \mathrm{z} 250), \mathrm{NOH}-\mathrm{PA}(\mathrm{m} / \mathrm{z} 252)$ and the oxidation product with $\mathrm{m} / \mathrm{z}, 143$ respectively. 
and human liver microsomes (HLM) respectively. The incubation mixtures have been analysed by LC/MS measurements. Extracted ion traces of PA and its metabolites are shown in figure 4.6a and $\mathrm{b}$. The structures of the metabolites are presented in figure 4.4. In the RLM incubation mixture, the metabolites NOH-PA $(\mathrm{m} / z 252)$ and NO-PA $(\mathrm{m} / \mathrm{z}, 250)$ were identified. In contrast, in the HLM incubation mixture the dealkylation product DEPA $(\mathrm{m} / \mathrm{z} 208)$ and the oxygenation product NOH-PA were detected. These results are in agreement with the metabolites of PA described in the literature [24]. The differences between the RLM and the HLM incubation mixtures are due to the varying distribution of isoforms of the enzym group cytochrome P450 in each organism. Besides that, the absence of the reactive metabolite NO-PA $(\mathrm{m} / \mathrm{z}$ 250 ) in the HLM incubation mixture may result from a protein binding of NO-PA to cellular proteins.

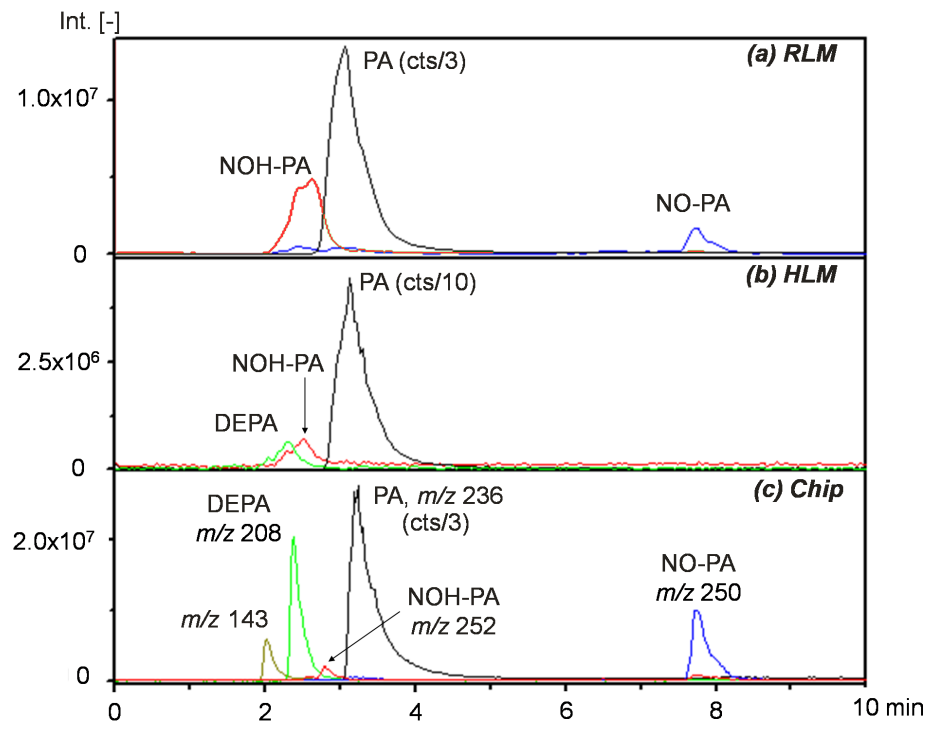

Figure 4.6: Extracted ion traces of PA and its oxidative metabolites DEPA, NO-PA, NOH-PA. (a) LC/MS analysis of the RLM incubation mixture of PA. (b) LC/MS analysis of the HLM incubation mixture of PA. (c) EC on-chip oxidation of the metabolism of PA $(1750 \mathrm{mV})$. Structures of the oxidation products are shown in fig. 4.4.

Using the on-line system consisting of the electrochemical cell, integrated on chip, the LC separation and subsequent MS detection, the metabolism of PA was simulated. Figure 4.6c shows the extracted ion traces of PA and its oxidation products, formed at a potential of $1750 \mathrm{mV}$ (vs. IrOx pseudo-reference) applied to the working 
electrode. The used flow rate was $0.125 \mu \mathrm{L} / \mathrm{min}$ over the main channel of the chip. The structures of the oxidation products are shown in figure 4.4. As can be seen from figure 4.6c, the formation of all three metabolites occurring in RLM and HLM (DEPA, NOH-PA, NO-PA) was successfully mimicked on-chip. Besides that, the oxidation product $m / z 143$ was formed. The proposed structure of this compound, shown in figure 4.7, indicates an oxidative cleavage in the benzylic position.

In order to ensure that the identified products derive from the oxidation on chip, blank measurements without applied potentials were performed. The dealkylation product DEPA was also present in the blank measurement but the intensities were approximately 10 times lower than at $1750 \mathrm{mV}$. The presence of DEPA in the blank sample might result from PA impurities or from air oxidation in the sample.

For identification of the oxidation products the exact masses were determined by time-of-flight mass spectrometry (ToF/MS). The deviation between the calculated and the measured $\mathrm{m} / \mathrm{z}$ was below $10 \mathrm{ppm}$ for all compounds. Moreover, fragmentation experiments (LC/MS/MS) of PA and its oxidation products have been performed. Figure 4.7 shows the fragmentation of PA and NOH-PA. All fragmentation patterns (product ion spectra) correlate to the structures shown in figure 4.4. 

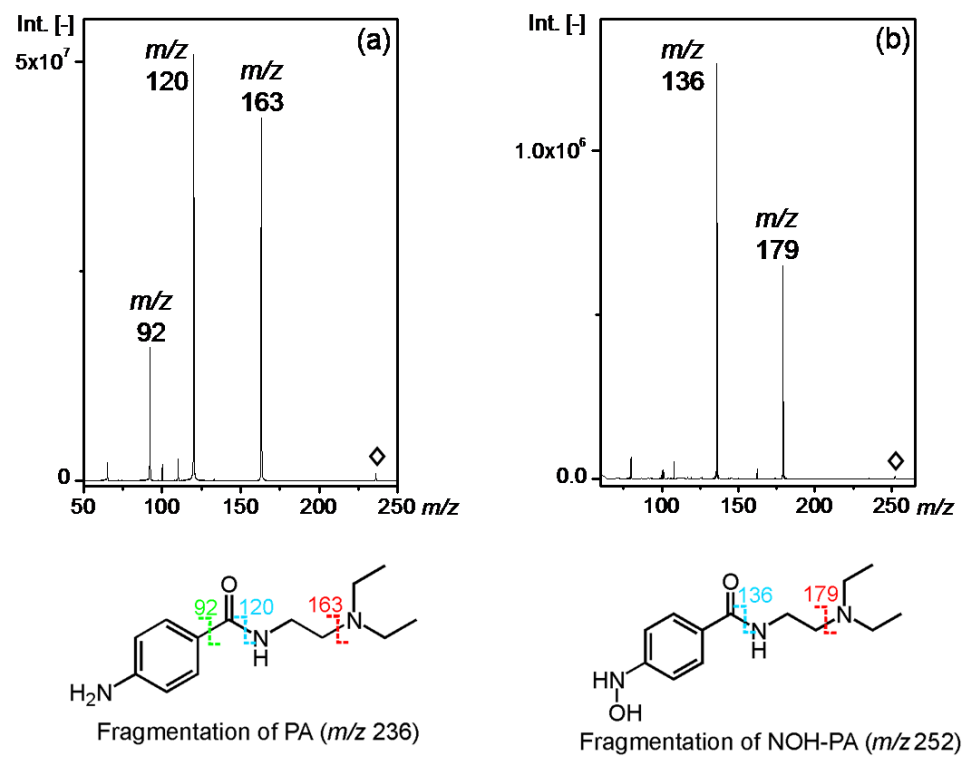

Figure 4.7: Product ion spectra of (a) PA and (b) NOH-PA, obtained by Chip/LC/MS/MS measurements. The fragmentation patterns correlate to the structures shown below the spectra. NOH-PA was formed on chip at a potential of 1750 $m V$ vs. IrOx pseudo-reference. 


\subsection{Conclusion}

An on-chip electrochemical cell was fabricated for routine use in drug metabolism studies. The electrochemical cell contains an integrated 3-electrode electrochemical cell with platinum working and counter electrodes and an $\mathrm{IrOx}$ pseudo-reference electrode. The internal volume of the main channel of this chip is $16 \mathrm{~nL}$ and the total internal volume of the chip is $180 \mathrm{~nL}$. The pseudo-reference electrode has a potential drift lower than $5 \mathrm{mV}$ during a typical drug conversion experiment (15 - 20 mins.). Therefore, the potential of the pseudo-reference electrode provides a stable operation for solutions with known and constant $\mathrm{pH}$. During the entire series of experiments no mechanical problems have been observed with the chip, even at high flow rates or acidic conditions.

The chip has been used routinely in our Lab-in-a-Briefcase instrument over the last year for the drug metabolism study of procainamide, a known antiarrythmic drug. The chip is used to mimic the oxidative mechanisms of the enzymes of the cytochrome P-450 family. The generated metabolites of rat and human liver microsomes are compared to the generated metabolites on-chip. The chip was able to generate all the known metabolites of both types of microsomes in a single experiment. Therefore, we have successfully mimicked the drug metabolism of procainamide in our electrochemical cell on-chip. In the case of procainamide, on-chip oxidation actually proves to give more information on the generated metabolites in a single experiment compared to microsomal studies. 


\section{Bibliography}

[1] M. Odijk, A.Baumann, W.Olthuis, A. den Berg, and U. Karst. Biosensors andBioelectronics, $\mathbf{x x x}: \mathrm{xxx}-\mathrm{xxx}, 2010$.

[2] F. Guengerich. Drug Metabolism in drug design and development. Wiley \& Sons inc., 2007.

[3] B. Park, N. Kitteringham, J. Maggs, M. Pirmohamed, and D. Williams. Annu Rev Pharmacol Toxicol, 45:177-202, 2000.

[4] R. Iyer and D. Zhang. Drug Metabolism in drug design and development. Wiley \& Sons Inc, 2007.

[5] E. Brandon, C. Raap, I. Meijerman, J. Beijnen, and J. Schellens. Toxicol Appl Pharmacol, 189:233-246, 2003.

[6] W. Lohmann and U. Karst. Anal Chem, 79:6831-6839, 2007.

[7] W. Lohmann, H. Hayen, and U. Karst. Anal Chem, 80:9714-9719, 2008.

[8] A. Baumann, W. Lohmann, B. Schubert, H. Oberacher, and U. Karst. J of Chromatogr A, 1216:3192-3198, 2009.

[9] S. van Leeuwen, B. Blankert, J. Kauffmann, and U. Karst. Analytical Bioanalytical Chemistry, 382:742-750, 2005.

[10] T. Johansson, L. Weidolf, and U. Jurva. Rap Comm Mass Spectrom, 21:23232331, 2007.

[11] A. Baumann and U. Karst. Expert Opin Drug Metab Toxicol, 6:715-731, 2010.

[12] W. Lohmann and U. Karst. Analytical Bioanalytical Chemistry, 391:79, 2008.

[13] H. Permentier, A. Bruins, and R. Bischoff. Mini-Rev Med Chem, 8:46-56, 2008.

[14] T. Nissila, L. Sainiemi, M.-M. Karikko, M. Kemell, M. Ritala, S. Franssila, R. Kostiainen, and R. Ketola. In Microtas conference, W72F. 2009.

[15] M. Odijk, A. Baumann, W. Lohmann, F. T. G. van den Brink, W. Olthuis, U. Karst, and A. van den Berg. Lab on a Chip, 9:1687-1693, 2009.

[16] D. J. Ives and G. J. Janz. Reference electrodes. Academic press, 1961.

[17] S. Carroll and R. Baldwin. Anal Chem, 82:878-885, 2010.

[18] M. Wang, S. Yao, and M. Madou. Sens Actuators B, 81:313-315, 2002.

[19] S. Yao, M. Wang, and M. Madou. Journal of the electrochemical society, 148:H29-H36, 2001.

[20] C. Li, P. Wu, W. Jung, C. Ahn, L. Shutter, and R. Narayan. 2009.

[21] C. Schabmueller, D. Loppow, G. Piechotta, B. Schutze, J. Albers, and R. Hintsche. Biosensors and Bioelectronics, 21(9):1770-1776, 2006. ISSN 0956-5663.

[22] H. Yang, S. K. Kang, C. A. Choi, H. Kim, D.-H. Shin, Y. S. Kim, and Y. T. Kim. Lab on Chip, 4:42-46, 2004. 
[23] D. Midgley. Talanta, 37(8):767-781, 1990. ISSN 0039-9140.

[24] H. Ha and F. Follath. Current Drug Metab, 5:543-571, 2004.

[25] A. J. Bard and L. R. Faulkner. Electrochemical methods - fundamentals and applications. Wiley, 2nd edition, 2001.

[26] W. Olthuis, M. Robben, P. Bergveld, M. Bos, and W. van der Linden. Sens Actuators B, 4:247-256, 1990.

[27] S. Marzouk, Ufer, R. Buck, T. Johnson, L. Dunlap, and W. Cascio. Anal Chem, 70:5054-5061, 1998. 



\section{Preliminary results of peptide cleavage on-chip}

In this chapter the latest results are presented of electrochemical peptide cleavage using a miniaturized electrochemical cell on-chip. This chip contains a complete three-electrode cell with platinum working and counter and iridium oxide pseudo reference electrodes and microfluidic channels with a total internal volume of $180 \mathrm{~nL}$. Using this chip, experiments have been conducted to achieve electrochemical oxidation and cleavage of the tripeptide Leucine-Tyrosine-Leucine. The results obtained with the chip are compared to results obtained with a commercially available cell with a porous graphite flow-through working electrode. Compared to the commercial cell, the chip showed a lower oxidation yield. The subsequent cleavage yield was also lower. Therefore, several modifications are proposed to improve future protein cleavage experiments using miniaturized electrochemical cells embedded in a microfluidic chip. ${ }^{1}$

\footnotetext{
${ }^{1}$ Julien Roeser and Alexander Louwes are gratefully acknowledged for the results presented in this chapter.
} 


\subsection{Introduction}

In the field of proteomics a complete set of proteins produced by an entire organism or system is studied. While the genome of an organism is more or less constant, the proteome varies considerably from organism to organism and even within time for a single organism. Therefore proteomics gained more scientific interest in the past two decades because it gives a better understanding of the functioning of an organism.

Peptide mapping is a widely used method in proteomics for its ability to identify unknown proteins. Proteins are cleaved into peptides and subsequently analyzed by mass spectrometry (MS). The resulting set of peptides is compared to a protein database to identify the unknown protein. Further information of the structure can be obtained by additional analysis of the protein fragments e.g. by MS/MS techniques $[1,2]$.

Peptide mapping relies on specific, and therefore predictable, cleavage of proteins. Enzymatic digestion of peptides is the most popular method. Trypsin is the most commonly used enzyme for its specific cleavage of peptide bonds on the carboxylic side between arginine or lysine with another amino acid 2 [1, 2]. Chemical cleavage of proteins can be used as an alternative to enzymatic digestion and offers the possibility to aim for different cleavage sites. One of the compounds probably most commonly used in chemical cleavage is cyanogen bromide, for its specific cleavage at the carboxylic side of methionine $[3,4]$. More compounds can be employed although it generally leads to overall lower cleavage yields [5, 6].

Direct electrochemical (EC) oxidation of peptides is another alternative method for specific protein cleavage. This method has been described only a few times in literature [7-11] and has gained more interest recently by our project partners from Groningen [12-14]. Electrochemical oxidation of peptides and proteins has been shown to allow specific cleavage of the peptide bonds next to tyrosine and tryptophan residues [14]. The main benefits of direct electrochemical oxidation rely in the speed of analysis and the possibility to couple the electrochemical cell directly on-line to a liquid chromatography (LC) - MS system. The entire setup is thus fully instrumental and allows full automation. However, the drawbacks of direct electrochemical oxidation are the rather low cleavage yields together with adsorption issues at the electrode surface when working with proteins with the commonly used carbon electrodes. Precise control of the experimental conditions such as $\mathrm{pH}$, electrode potential and solvent composition can also be an issue in some cases. The low cleavage yields are the result of competing non-cleavage reactions that occur after the first

\footnotetext{
${ }^{2}$ except if the other amino acid is proline
} 
oxidation step and accessibility of tyrosine and tryptophan residues which need to be in contact with the electrode surface to undergo oxidation and cleavage.

Permentier et al. $[12,13]$ have studied the protein cleavage of small $(1-14 \mathrm{kDa})$ to large $(17-67 \mathrm{kDa})$ proteins. In general cleavage occurs at almost every possible cleavage site for small proteins, while only some or no cleavage is observed for the larger proteins. The highest cleavage yield achieved to date is $80 \%$ reported by Roeser et al. [14] when using a small tripeptide glycine-tyrosine-glycine. The high cleavage yield is believed to be caused by the minimal steric hindrance of glycine ${ }^{3}$, which might also explain the absence of cleavage at possible cleavage sites for larger proteins in the study of Permentier et al. [13].

In the rest of this chapter, some preliminary results of attempts to cleave peptides using the electrochemical cell on-chip are presented. Although these first results indicate that we have achieved some cleavage, the results are not conclusive and by no means optimized. Therefore, the causes of what we believe to be the reason for the suboptimal results are discussed. Moreover, suggestions for further research are presented to improve this work in the future.

\subsection{Theory}

The reaction mechanism of oxidation and subsequent cleavage of tyrosine containing proteins as proposed in [12] is shown in figure 5.1. The initial peptide is shown in the upper left-corner, where $\mathrm{R}$ and $\mathrm{R}$ ' indicate the adjacent amino acids of the peptide. In an initial two electron oxidation process, one proton is abstracted from the phenolic side chain of tyrosine. The resulting phenoxonium ion is an electrophile intermediate which is not detected by the MS due to its high reactivity. The phenoxonium ion can undergo several secondary chemical reactions to form cleavage and non-cleavage oxidation products. A second proton can be abstracted from the phenolic ring to form the corresponding cyclohexadienone (1). Under abstraction of a proton and uptake of a water molecule (hydroxylation), the products ortho- (2) and parahydroxylated tyrosine (3) are formed. Product 3, the quinol product, can undergo further oxidation to form the corresponding quinone (4). The most important products in light of the application are the cleavage products formed by intramolecular reaction of the phenoxonium group with the carbonyl group at the $\mathrm{C}$-terminal side of tyrosine. By hydrolysis of the formed imine bond, cleavage of the peptide bond at the C-terminal to tyrosine is observed and yields a $\mathrm{N}$-terminal peptide with a spirolactone moiety (5) and an unmodified C-terminal peptide (6). A similar reaction yielding cleavage

\footnotetext{
${ }^{3}$ Glycine has no side-chain as shown in appendix A.3.
} 


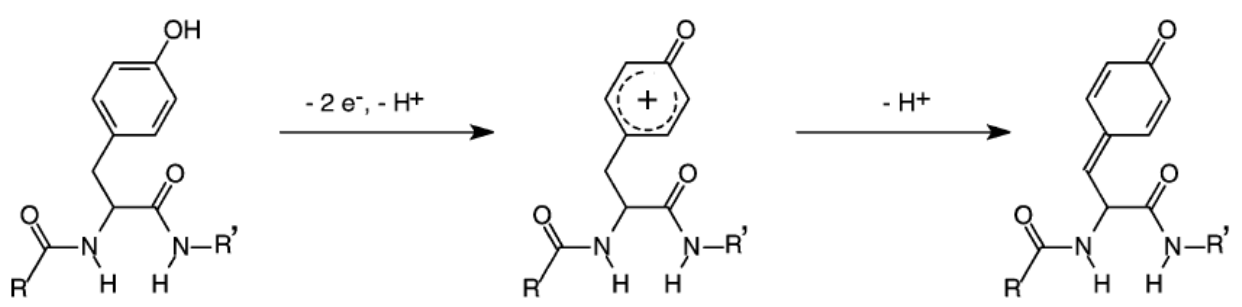

$M\left(R Y R^{\prime}\right)$

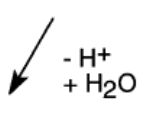

(1)

(2) $M+16$
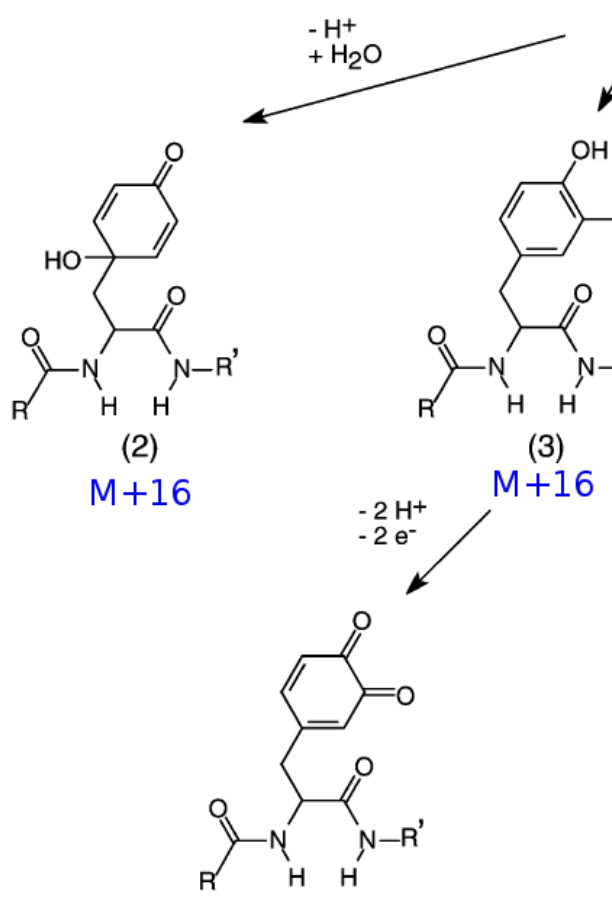

(4) $M+14$

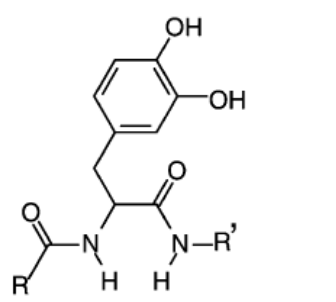

(3)

\section{$\mathrm{M}-2$}
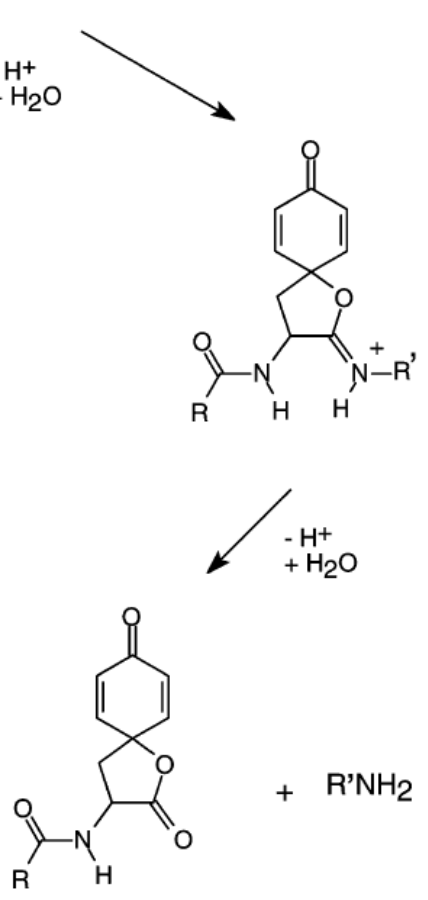

$$
\text { RY-2 (5) (6) R' }
$$

Figure 5.1: Proposed oxidation and cleavage mechanism for tyrosine containing peptides as described in [12]. $R$ and $R$ ' are the parts of the peptide $N$-terminal and $C$-terminal to the tyrosine residue, respectively. Upon oxidation of the phenol group of tyrosine, a phenoxonium group intermediate product is formed. This intermediate product undergoes further reactions to form non-cleavage products (1-4) and two cleavage products $(5+6)$. Relative masses are indicated in blue. 
of the tryptophanyl peptide bond is occurring when oxidizing tryptophan containing peptides [14], but this reaction is not discussed here.

The highest achieved cleavage yield is $80 \%$, for which a small tripeptide glycinetyrosine-glycine is used. The high cleavage yield is believed to be caused by the minimal steric hindrance of glycine, although that is not the only factor as high cleavage yields of approximately $75 \%$ with a tripeptide containing glutamic acid and tyrosine (E-Y-E) are also observed [14]. In the latter case the acidic environment might facilitate a good cleavage efficiency.

\subsection{Experimental}

\subsubsection{Electrochemical cell on-chip}

The miniaturized electrochemical cell on-chip used for the experiments described in this chapter is the same as used in chapter 4 and the corresponding paper [15]. Therefore, the details on the chip fabrication are already discussed in section 4.2 and in detail in appendix A.1. In short, two pyrex wafers are processed using standard lithography, (B)HF etching, sputtering and lift-off techniques to form a glass microfluidic chip with platinum working and counter and iridium oxide reference electrodes. A photo of this chip is shown in figure 5.2a.

\subsubsection{Chemicals}

The tripeptide Leucine-Tyrosine-Leucine (LYL) was chosen for comparison to the mass spectra obtained with commercially available electrochemical cells [14]. LYL was prepared in a solvent mixture of 90/10/1 (v/v/v) water/acetonitrile/formic acid at a concentration of $5 \mu \mathrm{M}$. LYL was purchased from Research Plus Inc. (Barnegat, NJ, USA). Formic acid was obtained from Sigma Aldrich (Steinheim, Germany). Water was purified by an Arium Ultrapure water system (conductivity $18.2 \mathrm{M} \Omega \mathrm{cm}$, Sartorius Stedim Biotech, Göttingen, Germany). HPLC supra gradient acetonitrile was purchased from Merck (Darmstadt, Germany).

\subsubsection{Methods}

The setup used consisted out of the earlier described Lab-in-a-Briefcase (see chapter 4.2 and the corresponding paper [15]). A photo of this setup is shown in figure 5.2b. In short, an automated setup built inside a briefcase contains two independent syringe pumps which drive the fluid flow through the microfluidic chip. Analyte is introduced through the inlet of the chip at a flow rate of $0.25 \mu \mathrm{L} / \mathrm{min}$. An outgoing flow 
of $0.125 \mu \mathrm{L} / \mathrm{min}$ is enforced on the outlet of the side-channel of the chip. The resulting flow through the main channel, where oxidation is taking place is $0.125 \mu \mathrm{L} / \mathrm{min}$. An oxidation potential of $2 \mathrm{~V}$ (vs. IrOx pseudo-reference) is applied to the working electrode inside the chip using a portable potentiostat. The resulting oxidation products are collected in a $5 \mu \mathrm{L}$ sample loop which was filled for $64 \min (8 \mu \mathrm{L})$ to ensure that the loop was completely filled with oxidation products from the chip. After switching an injection valve, the contents of the sample loop are directly flushed into an API365 triple quadrupole mass spectrometer (MDS-Sciex, Concord, Ontario, Canada; upgraded to EP10+, Ionics, Bolton, Ontario, Canada; equipped with a TurboIonSpray source) at a flow rate of $5 \mu \mathrm{L} / \mathrm{min}$ using a third (external) syringe pump (KD Scientific Inc., Holliston, MA) and the same solvent mixture as described in section 5.3.2. The MS and the third syringe pump are not included in figure 5.2b.

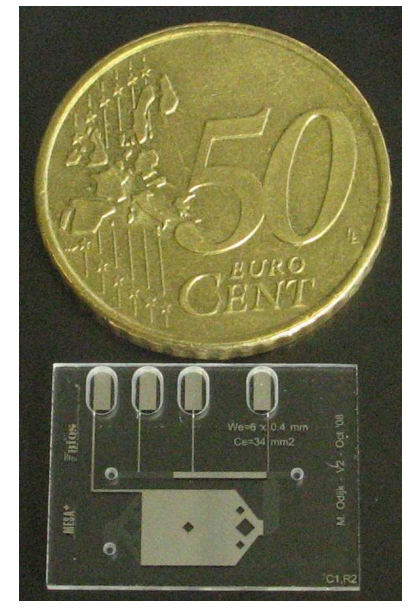

(a) Microfluidic chip

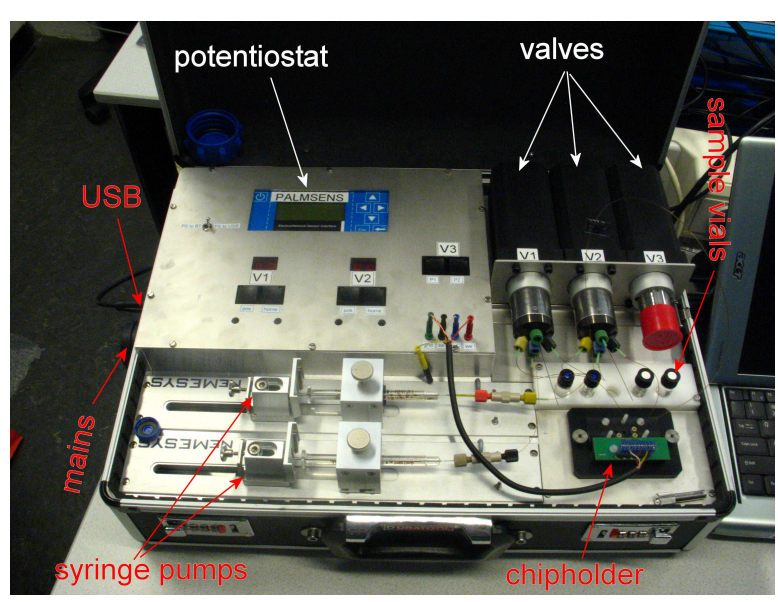

(b) Lab-in-a-Briefcase setup

Figure 5.2: (a) Electrochemical cell on-chip and (b) Lab-in-a-Briefcase setup as used in the experiments shown in this chapter.

For the control experiment with the commercially available EC cell, a Coulochem 5021 conditioning cell was used (ESA Inc., Bedford, MA) containing a porous graphite flow-through working electrode and $\mathrm{Pd} / \mathrm{H}_{2}$ working and counter electrode. This cell was used at $1000 \mathrm{mV}$ working electrode potential (vs. $\mathrm{Pd} / \mathrm{H}_{2}$ ) applied by an in-house developed potentiostat. Analyte was introduced into the cell at $50 \mu \mathrm{L} / \mathrm{min}$ using a syringe pump (KD Scientific Inc., Holliston, MA). The analyte used in the control experiment consisted of the same solvent mixture $(90 / 10 / 1(\mathrm{v} / \mathrm{v} / \mathrm{v})$ water/acetonitrile/formic acid) and a concentration of $2 \mu \mathrm{M} \mathrm{LYL}$. 


\subsection{Results and discussion}

In figure 5.3 compares the results obtained after electrochemical oxidation of LYL performed with the EC cell on-chip (fig. 5.3a) and the commercially available EC cell (fig. 5.3b). Overall signal intensity is approximately 10-fold lower for the chip, due to a 10-fold lower flow rate used at the electrospray ionization (ESI) interface. Both cells yielded the range of products 1-5 indicated in figure 5.1. In the case of the commercial cell, the starting compound LYL $(\mathrm{m} / \mathrm{z}, 408)$ is not detected, indicating that oxidation yield is near to $100 \%$. The cleavage products LY-2 $(\mathrm{m} / \mathrm{z} 293)$ and $\mathrm{L}(\mathrm{m} / \mathrm{z}$ 131) are present in high amounts with a cleavage yield of approximately 40-50\%. The main non-cleavage products annotated M-2 (1), M+14 (4) and M+16 (2-3) are also observed with high intensities. If this result is compared to the results obtained with the chip, LYL is the most abundant ion observed in the spectrum, indicating only moderate oxidation yield. As a consequence, the corresponding reaction products are only observed in low yields. The presence of the LY-2 product in the spectrum indicates that some cleavage occurring in the EC cell on-chip as well. C-terminal cleavage product $\mathrm{L}$ is not visible in this spectrum since a limited mass range $(\mathrm{m} / \mathrm{z}$ 250-500) was chosen in this experiment in order to work with a longer scanning time allowing an increased sensitivity. Finally, some products with mass $\mathrm{M}+32$ and $\mathrm{M}+48$ are measured, which are believed to be further hydroxylation products of the $\mathrm{M}+16$ product. Since overall signal intensities for the oxidation products are just above the noise level, the results obtained with the chip are not conclusive.

Several actions can be taken to improve the results obtained with the chip. To increase overall signal intensities, the concentration can be increased. However, a higher concentration will facilitate dimer formation and will not lead to a higher cleavage yield. Another way to increase signal intensity, is to use a higher flow rate to flush the sample loop, although this requires a larger sample loop to avoid a too short injection plug that would hamper MS detection. A larger sample loop requires more time to fill by the chip, resulting in impractical filling times (several hours). Simply filling the loop at a higher rate is also not possible, since the overall oxidation yield is already lower than desired.

The best setup for this experiment using the current chip design is to use another MS capable of nanospray ESI at a higher sensitivity. Commercially available systems providing nanospray typically work in the range of $100-200 \mathrm{~nL} / \mathrm{min}$, which matches the flow rates used inside the chip more closely.

Another approach is to perform a complete redesign of the chip, for the specific purpose of protein cleavage. Using the theory as described in section 3.2, it is possible to 


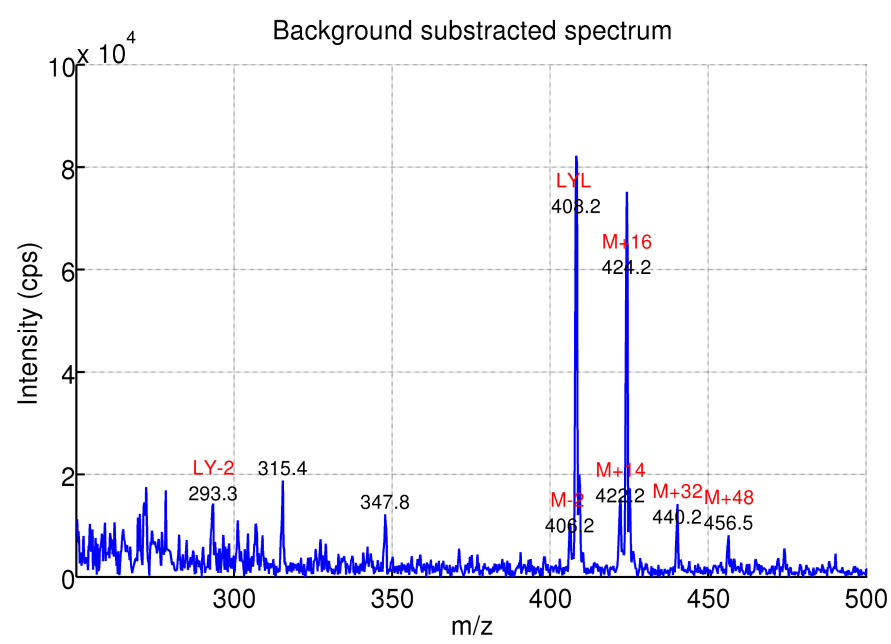

(a) Microfluidic EC chip

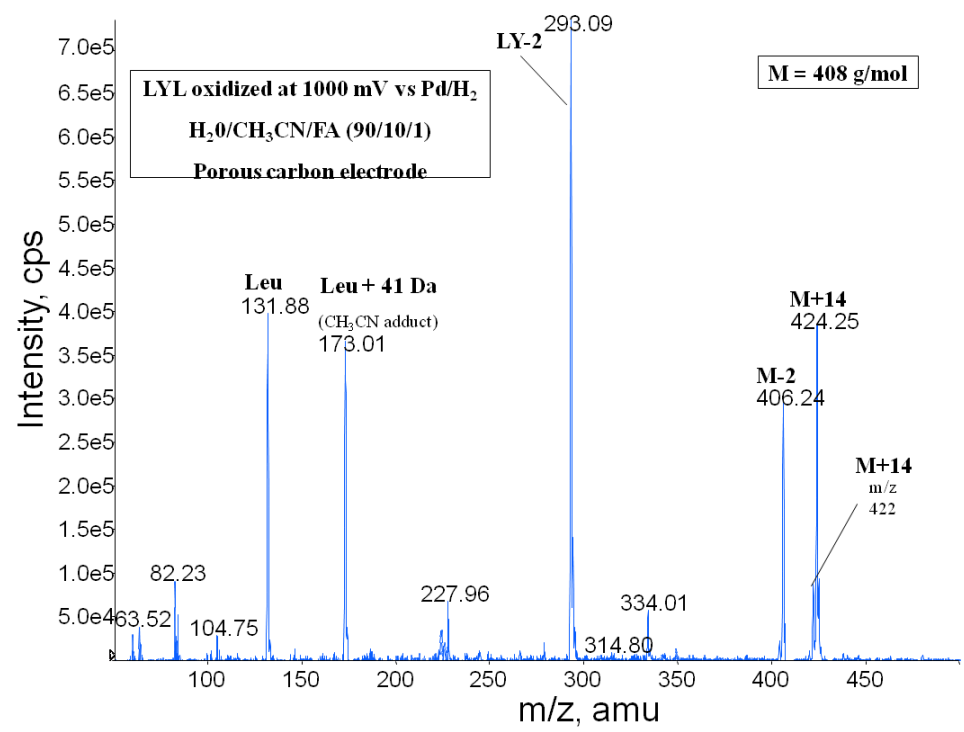

(b) Commercially available EC cell with porous glassy carbon electrode

Figure 5.3: Mass spectrograms of electrochemically cleaved LYL. Important peaks are indicated in the figure like the starting product $(\mathrm{m} / \mathrm{z} 408.2)$, the cleavage product $(\mathrm{m} / \mathrm{z}, 293.3)$ and non cleavage products $(\mathrm{m} / \mathrm{z}$ 406.2, 422.2, 424.2, 440.2 and 456.5) as explained in figure 5.1. 
optimize the chip for higher flow rates, while maintaining a good theoretical oxidation yield. However, in light of the oxidation yields achieved in figure 5.3a, the theory described in section 3.2 should be enhanced, including the mass transport and more importantly, the reaction kinetics of proteins. The major benefit of a redesign of the chip is the possibility of changing the working electrode material. Experiments with commercially available cells containing platinum working electrodes suffer from irreversible adhesion of proteins to the electrode surface. Although these effects are not observed during the experiments presented here, carbon or diamond-like electrode surfaces might be much better candidates for (good) repeatable protein cleavage experiments $[16,17]$. A complete redesign of the chip, including fabrication and possibly new electrode material characterization is a time consuming process and is therefore not possible to conduct within the remaining time of this project.

\subsection{Conclusion}

In this chapter, results of electrochemical peptide cleavage on-chip are compared to results obtained with commercially available electrochemical flow-through cells. Some peptide cleavage on-chip is observed, but the overall oxidation and cleavage yields were low. The results obtained using the commercially available cells were much better. Overall signal intensities measured using the chip were also low, due to a flow rate mismatch between the chip and the ESI-MS system.

Chip technology might promise (disposable) cells and automated systems for protein cleavage in the future with the possibility to reduce the required sample volume and associated costs. To fulfill this promise, further system optimization is essential. On the one hand a complete redesign of the chip is desired with an optimized geometry and different working electrode materials to prevent protein adhesion. On the other hand, additional equipment needs to be optimized as well e.g. by using a nanospray MS system to be able to work with small sample volumes. 


\section{Bibliography}

[1] D. Liebler. Introduction to Proteomics. ISBN 0-89603-992-7. Humana Press, 2002.

[2] N. C. Mishra. Introduction to Proteomics - Principles and applications. ISBN 0471754021. Wiley, 2010.

[3] B. Witkop. Science, 162:318-326, 1968.

[4] D. J. Walton, P. G. Richards, J. Heptinstall, and B. Coles. Electrochimica Acta, 42(15):2285-2294, 1997.

[5] D. Crimmins, S. Mische, and N. Denslow. Current protocols in protein science, 11.4, 2005. doi:10.1002/0471140864.ps1104s40.

[6] J. Roeser, R. Bischoff, A. Bruins, and H. Permentier. Anal Bioanal Chem, 397:3441-3455, 2010.

[7] B. Malfoy and J. A. Reynaud. J Electroanal Chem, 114(2):213 - 223, 1980. ISSN 0368-1874. doi:10.1016/0368-1874(80)80376-5.

[8] J. Reynaud, B. Malfoy, and A. Bere. J Electroanal Chem, 116:595 - 606, 1980. ISSN 0022-0728. doi:10.1016/S0022-0728(80)80281-6.

[9] H. Iwasaki, L. A. Cohen, and B. Witkop. Journal of the American Chemical Society, 85(22):3701-3702, 1963. doi:10.1021/ja00905a039.

[10] L. Farber and L. A. Cohen. Biochemistry, 5(3):1027-1034, 1966. doi:10.1021/ bi00867a031.

[11] S. M. MacDonald and S. G. Roscoe. Electrochimica Acta, 42(8):1189 - 1200, 1997. ISSN 0013-4686. doi:10.1016/S0013-4686(96)00285-X.

[12] H. Permentier, U. Jurva, B. Barroso, and A. Bruins. Rap Comm in Mass Spectrometry, 17(14):1585-1592, 2003.

[13] H. P. Permentier and A. P. Bruins. Journal of the American Society for Mass Spectrometry, 15(12):1707 - 1716, 2004. ISSN 1044-0305. doi:10.1016/j. jasms.2004.09.003.

[14] J. Roeser, H. Permentier, A. Bruins, and R. Bischoff. Anal Chem, 82:7556$7565,2010$.

[15] M. Odijk, A.Baumann, W.Olthuis, A. den Berg, and U. Karst. Biosensors andBioelectronics, $\mathbf{x x x}: \mathbf{x x x}-\mathbf{x x x}, 2010$.

[16] M. Fryda, T. Matthee, S. Mulcahy, A. Hampel, L. Schafer, and I. Troster. Diamond and Related Materials, 12:1950-1956, 2003.

[17] A. Kraft. Int J Electrochem Sci, 2:355-385, 2007. 


\section{Redox cycling and differential cyclic voltammetry}

Redox cycling $(R C)$ is a technique that is used to amplify electrochemical signals. In this chapter, Finite Element Method (FEM) simulation results of interdigitated array (IDA) electrode geometries are presented to study and verify redox selectivity and $R C$ amplification. These results provide an adequate explanation of an earlier found, but poorly understood, high amplification factor (65x) in a $1 \mu$ m-spaced IDA microdevice.

Using the FEM calculations we present selectivity measurements with IDA electrodes in a mixture of two redox species. We show that it is possible to electrochemically detect dopamine in the presence of the stronger reductor ferricyanide, which is impossible with direct amperometric detection. Using our simulations, we show that a theoretical selectivity of dopamine over ferricyanide of 11 can be achieved. More importantly we introduce a new measurement technique which delivers electrochemical spectra of all reversible redox couples present based on concentration and standard potentials. This technique has been named differential cyclic voltammetry (DCV).

This new DCV technique is experimentally verified using a fabricated micrometersized IDA electrode to conduct DCV measurements in mixtures of $1 \mathrm{mM}$ catechol and $\left.4 m M\left[R u\left(\mathrm{NH}_{3}\right)_{6}\right] \mathrm{Cl}_{3}\right]$. The experimental data corresponds to the calculated spectra obtained from simulations. Additionally, the measured spectra can be used to easily derive standard potentials and concentrations simultaneously and selectively. ${ }^{1}$

\footnotetext{
${ }^{1}$ Modified from: a paper published in Electroanalysis [1] and an extended abstract submitted to the IEEE Sensors conference 2010. Co-authors are J. Wiedemair, M.J.J. van Megen, W. Olthuis, V.A.T. Dam and A. van den Berg.
} 


\subsection{Introduction}

Redox cycling (RC) is a technique used in electrochemical detection of redox active species, amongst other leading to a better detection limit. With this method it is even possible to achieve single molecule detection [2]. The effect is observed when two electrodes are placed in close proximity of each other such that their concentration profiles overlap. As illustrated in figure $6.1 \mathrm{~b}$, the ion involved in a reversible redox reaction can cycle between a generator and collector electrode. Each cycle contributes one electron to the measured current, effectively amplifying the measured current in a chemical manner ${ }^{2}$. Redox cycling can be used for electrochemical amplification of the measured current. One of the electrode structures frequently used to achieve high redox cycling is the interdigitated array (IDA) electrode (see fig. 6.1a).

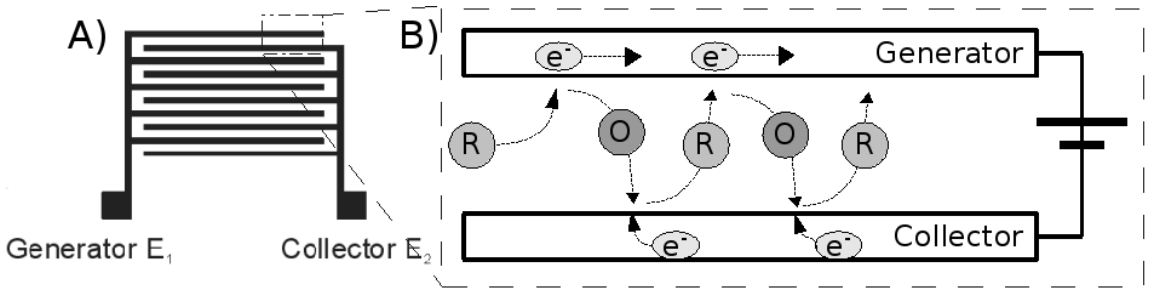

Figure 6.1: A) Top view of an interdigitated array electrode. B) Redox cycling illustrated between two facing electrodes.

The redox cycling effect has been a major topic of research in literature since the pioneering work of Sanderson and Anderson in 1985 [3]. Most papers on RC make use of an interdigitated array electrode [3-17]. The group of Paeschke et al. [11] reported $\mathrm{RC}$-amplification of 14 times achieved with redox cycling at an IDA-electrode. Ueno et al. [17] presented a RC-amplification of up to 40x with IDA electrodes with nanometer dimensions. Also, Niwa et al. [9] used an IDA-electrode to achieve a RC-amplification of approximately 45x. An amplification of 100 times is reported for IDA electrodes used in nanochannels [18]. An even higher RC-amplification of approximately 400x is reported by Zevenbergen et al. [19], for which he used a parallel plate configuration spaced at $300 \mathrm{~nm}$ apart.

Using the same electrode structure as Zevenbergen et al.[19], Wolfrum et al. [20] reports cyclic voltammetry (CV) measurements where only 265 molecules are involved. During scanning electrochemical microscopy (SECM) the so-called feedback mode uses the RC effect to amplify the current measured between a disk-shaped

\footnotetext{
${ }^{2}$ In the case of a single electron transfer reaction.
} 
ultramicroelectrode and a (biased) substrate. Using this method Fan and Bard [2] report the detection of single molecule activity.

It is feasible to achieve selective detection using RC since only the current of cycling species is amplified. For example, it is shown that small amounts of catechol can be detected in the presence of interfering species such as ascorbic acid [20]. Both ascorbic acid and catechol are oxidized easily, however only catechol forms a reversible redox couple with quinone. Therefore, only the current measured from catechol conversion is amplified by RC.

The redox cycling effect is also studied extensively within our group by V.A.T. Dam et al. [21]. We observed a RC-amplification of $65 x$ with an IDA-electrode. In this chapter, we present an explanation for this unexpectedly high RC-amplification. Also, we report a method to use the RC effect to increase selectivity among a selected set of redox active species. This method is based on a fixed potential applied to one electrode, while the potential of the other electrode is controlled using CV. These selectivity simulations are compared to the experimental data as published by V.A.T. Dam et al. [21]. Data obtained using the fixed potential method is often not as conclusive as desired, since it is difficult to obtain direct information on the concentrations of the separate species present.

To overcome these issues, we present a novel measurement technique in this chapter which we have named differential cyclic voltammetry (DCV). DCV is based on RC, and delivers immediate information on all reversible redox species present within the solution. The resulting data resembles a differential pulse voltammogram (DPV) or the electrochemical equivalent of a mass spectrogram. On the x-axis (unit: volt) the position of the peaks indicate the standard potential of a reversible redox couple, whereas on the $y$-axis (unit: ampere) the concentration of this couple is indicated by the peak height. Compared to DPV DCV has the added benefit of more selectivity and a simpler potential waveform. We have tested this new technique both theoretically and practically using a finite element model and an IDA electrode. Other papers have been published describing models capable of simulating RC at IDA electrodes [4, 12, 22-24]. Additionally, we present simulation results on the selectivity achieved with redox cycling and the new DCV measurement technique.

\subsection{Theory}

\subsubsection{Governing equations}

The software used for the finite element modelling is the Multhiphysics package from the company Comsol. The basic governing equations used in the FEM model 
are the Nernst-Planck equation and the continuity equation. Diffusion is assumed as the only means of mass transport, which is valid if geometric dimensions of the gap between the electrodes is much larger than the Debye-length and sufficient excess supporting electrolyte is present. Therefore mass transport of ions is only diffusive in nature and Ficks second law is applicable:

$$
\frac{\partial C_{j}}{\partial t}=D_{j} \nabla^{2} C_{j}
$$

in which $C_{j}$ is the concentration in $\mathrm{mol} / \mathrm{m}^{3}$ and $D_{j}$ is the diffusion constant of redox-active species $j$. Furthermore it is assumed that the redox reactions in our model are reversible. In that case the general forward and backward reaction of a redox-couple can be described by:

$$
O+z \cdot e^{-} \underset{k_{b}}{\stackrel{k_{f}}{\rightleftharpoons}} R
$$

where $\mathrm{z}$ indicates the number of electrons involved during oxidation or reduction of a single electroactive ion. The forward and backward reaction rate constants $k_{f}$ and $k_{b}$ (in $\mathrm{m} / \mathrm{s}$ ) are described by the Butler-Volmer equations [25]:

$$
\begin{gathered}
k_{f}=k_{s} e^{-\alpha\left(E_{a p p l}-E^{0}\right) \frac{F}{R T}} \\
k_{b}=k_{s} e^{(1-\alpha)\left(E_{a p p l}-E^{0}\right) \frac{F}{R T}}
\end{gathered}
$$

with $k_{s}$ the standard rate constant, $\alpha$ the transfer coefficient, $E^{0}$ the standard potential of redox-active species and $E_{a p p l}$ the potential applied to the electrode. F, R and T are the Faraday constant, the gas constant and the temperature respectively. For redox couples with a two electron transfer reaction it is assumed that one of the electrons transferred determines the overall reaction rate, thus $\mathrm{z}$ is not included in equations 6.3 and $6.4[25]$.

The values for the constants used in all our simulations are listed in table 6.1. Four reversible redox couples (sp.1, sp.2, sp.3 and sp.4) are used with standard potentials, diffusion coefficients, and rate constants matching ferrocyanide, dopamine, $\mathrm{Ru}\left(\mathrm{NH}_{3}\right)_{6}$ and catechol respectively. These constants are found in various papers $[9,12,20,21,26-31]$ of which references are also included in the table. All potentials mentioned in table 6.1 are defined versus a standard silver/silverchloride reference electrode. 
Table 6.1: Constants used in all simulations

\begin{tabular}{|c|c|c|}
\hline \multicolumn{3}{|c|}{ Ferrocyanide - ferricyanide couple } \\
\hline$D_{1 R}$ - Diffusion coefficient reduced sp.1. $[9,12]$ & $7 \mathrm{E}-10$ & {$\left[\mathrm{~m}^{2} / \mathrm{s}\right]$} \\
\hline$D_{1 O}$ - Diffusion coefficient oxidized sp.1. $[9,12]$ & 7E-10 & {$\left[\mathrm{m}^{2} / \mathrm{s}\right]$} \\
\hline$C_{1 R}^{*}$ - Bulk concentration, reduced sp.1. & 5 & {$\left[\mathrm{~mol} / \mathrm{m}^{3}\right]$} \\
\hline$C_{1 O}^{*}$ - Bulk concentration, oxidized sp. 1. & 5 & {$\left[\mathrm{~mol} / \mathrm{m}^{3}\right]$} \\
\hline$k_{s 1}-$ Rate constant, redox couple 1 & $1.5 \mathrm{E}-4$ & {$[\mathrm{~m} / \mathrm{s}]$} \\
\hline$\alpha_{1}-$ Transfer coefficient, redox couple 1 & 0.5 & \\
\hline$E_{1}^{0}$ - Standard potential, redox couple $1[21,29]$ & 0.265 & {$[V]$} \\
\hline$z_{1}$ - Number of electrons transferred per sp. 1. & 1 & \\
\hline \multicolumn{3}{|c|}{ Dopamine - dopamine quinone couple } \\
\hline$D_{2 R}$ - Diffusion coefficient reduced sp.2. [30] & $9 \mathrm{E}-10$ & {$\left[\mathrm{~m}^{2} / \mathrm{s}\right]$} \\
\hline$D_{2 O}$ - Diffusion coefficient oxidized sp.2. [30] & $9 \mathrm{E}-10$ & {$\left[\mathrm{~m}^{2} / \mathrm{s}\right]$} \\
\hline$C_{2 R}^{*}$ - Bulk concentration, reduced sp.2. & 5 & {$\left[\mathrm{~mol} / \mathrm{m}^{3}\right]$} \\
\hline$C_{2 O}^{*}$ - Bulk concentration, oxidized sp. 2. & 5 & {$\left[\mathrm{~mol} / \mathrm{m}^{3}\right]$} \\
\hline$k_{s 2}$ - Rate constant, redox couple 2 & $1.5 \mathrm{E}-4$ & {$[\mathrm{~m} / \mathrm{s}]$} \\
\hline$\alpha_{2}-$ Transfer coefficient, redox couple 2 & 0.5 & \\
\hline$E_{2}^{0}$ - Standard potential, redox couple $2[21,29]$ & 0.5 & {$[V]$} \\
\hline$z_{2}$ - Number of electrons transferred per sp.2. & 1 & \\
\hline \multicolumn{3}{|c|}{$\mathrm{Ru}\left(\mathrm{NH}_{3}\right)_{6}^{3+}-\mathrm{Ru}\left(\mathrm{NH}_{3}\right)_{6}^{2+}$ couple } \\
\hline$D_{3 R}$ - Diffusion coefficient reduced sp.3. [26] & $7.5 \mathrm{E}-10$ & {$\left[\mathrm{~m}^{2} / \mathrm{s}\right]$} \\
\hline$D_{3 O}$ - Diffusion coefficient oxidized sp.3. [26] & $7.5 \mathrm{E}-10$ & {$\left[\mathrm{~m}^{2} / \mathrm{s}\right]$} \\
\hline$C_{3 R}^{*}$ - Bulk concentration, reduced sp. 3. & 0 & {$\left[\mathrm{~mol} / \mathrm{m}^{3}\right]$} \\
\hline$C_{3 O}^{*}$ - Bulk concentration, oxidized sp. 3 . & 4 & {$\left[\mathrm{~mol} / \mathrm{m}^{3}\right]$} \\
\hline$k_{s 3}-$ Rate constant, redox couple $3[31]$ & $3 \mathrm{E}-3$ & {$[\mathrm{~m} / \mathrm{s}]$} \\
\hline$\alpha_{3}-$ Transfer coefficient, redox couple 3 & 0.5 & \\
\hline$E_{3}^{0}$ - Standard potential, redox couple $3[26]$ & -0.16 & {$[V]$} \\
\hline$z_{3}-$ Number of electrons transferred per sp. 3 . & 1 & \\
\hline \multicolumn{3}{|c|}{ Catechol - 1,2-benzoquinone couple } \\
\hline$D_{4 R}$ - Diffusion coefficient reduced sp.4. [27] & $9 \mathrm{E}-10$ & {$\left[\mathrm{~m}^{2} / \mathrm{s}\right]$} \\
\hline$D_{4 O}$ - Diffusion coefficient oxidized sp.4. [27] & $9 \mathrm{E}-10$ & {$\left[\mathrm{~m}^{2} / \mathrm{s}\right]$} \\
\hline$C_{4 R}^{*}$ - Bulk concentration, reduced sp. 4. & 0 & {$\left[\mathrm{~mol} / \mathrm{m}^{3}\right]$} \\
\hline$C_{4 O}^{*}$ - Bulk concentration, oxidized sp. 4. & 1 & {$\left[\mathrm{~mol} / \mathrm{m}^{3}\right]$} \\
\hline$k_{s 4}$ - Rate constant, redox couple 4 [28] & $4.65 \mathrm{E}-4$ & {$[\mathrm{~m} / \mathrm{s}]$} \\
\hline$\alpha_{4}-$ Transfer coefficient, redox couple 4 & 0.5 & \\
\hline$E_{4}^{0}$ - Standard potential, redox couple 4 [20] & 0.2 & {$[V]$} \\
\hline$z_{4}$ - Number of electrons transferred per sp.4. & 2 & \\
\hline $\mathrm{R}$ - Gas constant & 8.31 & {$[J / K]$} \\
\hline $\mathrm{T}$ - Temperature & 290 & {$[K]$} \\
\hline F - Faraday constant & 96485 & {$[\mathrm{C} / \mathrm{mol}]$} \\
\hline $\mathrm{v}$-Scan rate & 50 & {$[\mathrm{mV} / \mathrm{s}]$} \\
\hline
\end{tabular}




\subsubsection{Geometry}
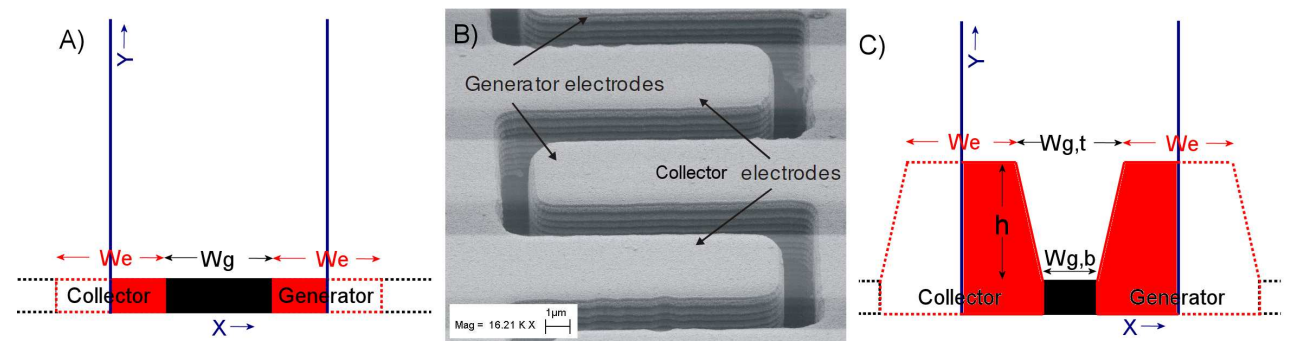

Figure 6.2: A) Cross-sectional view of an IDA with flat electrodes. B) SEM picture of the IDA-electrode used by V.A.T. Dam [21]. C) Estimated IDA geometry with raised electrodes derived from the SEM picture and the specifications supplied by V.A.T. Dam. Colors in figures A and B represent; red - electrodes, blue - lines of symmetry, black - substrate. Solid lines indicate the simulated geometry, dashed lines indicate the repeating nature of the IDA-electrode. The parameters are explained in the text.

We have used two different geometries in our simulations. The first geometry of an IDA electrode with its electrodes in plane with the substrate is indicated in figure 6.2a. The second geometry (figure 6.2c) is derived from the IDA-electrode used in the experiments of V.A.T. Dam [21]. As it can be seen from the SEM-picture shown in figure $6.2 \mathrm{~b}$, this second geometry contains raised electrodes with a trench in between the collector and generator electrode. In the cross-sectional views of the IDA, the generator and collector electrodes are indicated by the red areas. The blue lines indicate the planes of symmetry. We use two-dimensional models of only the parts drawn by solid lines, to decrease the computing time and necessary computer memory. Thus, only one finger pair of the IDA electrode is simulated. This model simplification is valid if the number of fingers is sufficiently large (more than 10). Three important parameters describing the dimensions of the geometry are the electrode width $\left(W_{e}\right)$, the gap width $\left(W_{g}\right)$ and the electrode height $(h)$. For the IDA with raised electrodes the gap width at the top $\left(w_{g, t}\right)$ and bottom $\left(w_{g, b}\right)$ are defined individually.

The following boundary conditions are used for the flux $(\mathrm{N})$ at the generator and collector electrodes:

$$
\begin{array}{r}
N_{g e n, o x}=-N_{g e n, r e}=k_{f} \cdot C_{o x}(t)-k_{b} \cdot C_{r e}(t) \\
N_{c o l, r e}=-N_{c o l, o x}=k_{f} \cdot C_{r e}(t)-k_{b} \cdot C_{o x}(t)
\end{array}
$$

At insulating walls, or planes of symmetry the normal flux is assumed to be zero:

$$
N_{i n s, j}=\vec{n} \cdot\left(-D_{j} \nabla C_{j}\right)=0
$$


where $\vec{n}$ is a vector perpendicular to the plane. The reduced and oxidized species concentration is kept at the initial bulk concentration $\left(C_{j}^{*}\right)$ at a parallel plane to and far away from the substrate, thus creating a sort of semi-infinite bulk boundary (SIBB) condition:

$$
C_{j, S I B B}=C_{j}^{*}
$$

Usually the position of the SIBB can be estimated by:

$$
y_{\text {top }}=\sqrt{4 \cdot D_{\max } \cdot t_{t o t}}
$$

where $y_{\text {top }}$ is the distance between the plane of the electrodes and the bulk boundary condition, $t_{t o t}$ the total simulation time and $D_{\max }$ the fastest diffusion coefficient of all ions present. This SIBB condition is valid under certain circumstances which are further explained in the results section.

\subsubsection{Applied potentials}

Except for the selectivity simulations, we only use the ferro/ferricyanide redox couple in our simulations and generator and collector potentials are set to $-0.2 \mathrm{~V}$ and $0.7 \mathrm{~V}$ respectively.

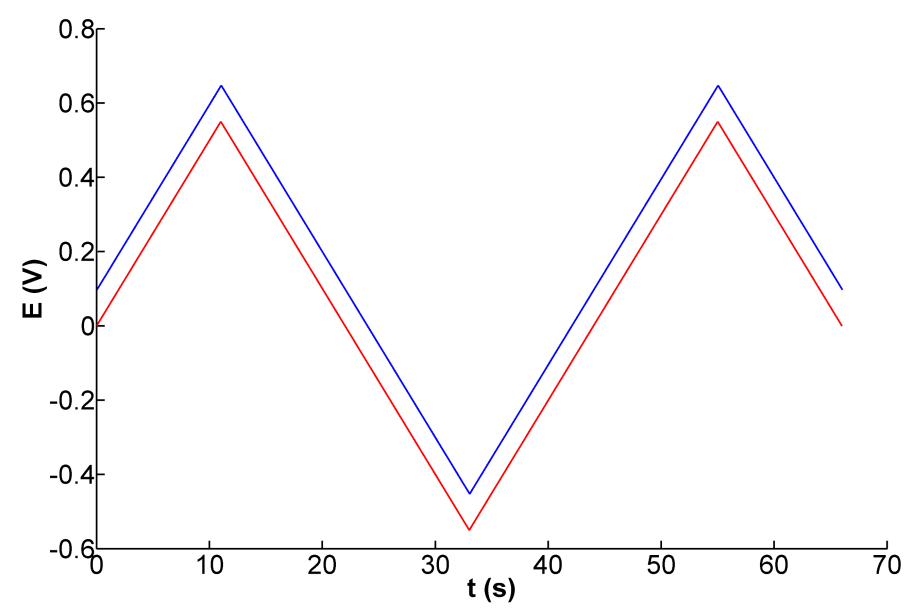

Figure 6.3: Potentials applied to both electrodes as used in the DCV technique. A fixed offset of, in this case $100 \mathrm{mV}$, is maintained.

The key point for using DCV is the application of appropriate potentials to both electrodes. These potentials are illustrated in figure 6.3. The potential of one electrode is 
shown in red, and the potential waveform of the other electrode in blue. Between the two waveforms a fixed offset is maintained. The value of this offset influences the peak width observed in the data obtained using DCV. The optimal offset for maximized resolution is related to the peak separation in conventional CV, which is [25]:

$$
\Delta E_{p}=\frac{2.2 \cdot R \cdot T}{z \cdot F}
$$

For a single electron transfer reaction at $25^{\circ} C \Delta E_{p}$ equals $57 \mathrm{mV}$.

\subsubsection{Post-processing}

The following equation describes the relation between the current from the electrodes and the flux at the electrode:

$$
N_{j}=\frac{i_{j}}{z_{j} F A}=\vec{n} \cdot\left(D_{j} \nabla C_{j}\right)
$$

In most simulations this flux is not homogeneous over the entire surface of the electrode. To get correct values for the current it is necessary to integrate the flux over the entire path $(l)$ along the electrode surface. In $2 \mathrm{D}$ simulations the result is a current with dimensions $\mathrm{A} / \mathrm{m}$ calculated using the following relation:

$$
i_{j}=z_{j} \cdot F \int \vec{n} \cdot\left(D_{j} \nabla C_{j}\right) d l
$$

Subsequently, the collection efficiency (CE) and the number of redox cycles (RC) are calculated using equation 6.13 and 6.14 as given by Niwa et al. [9].

$$
\begin{array}{r}
C E=\frac{i_{c}}{i_{g}} \\
R C=\frac{1}{1-C E^{2}}
\end{array}
$$

In equation $6.13, i_{c}$ and $i_{g}$ are the collector and generator current respectively.

\subsection{Experimental}

\subsubsection{Sensor fabrication}

The IDA electrodes are fabricated using conventional lithography and lift-off processes. A 550nm layer of lift-off resist (LOR5a, Microchem) and $1.7 \mu \mathrm{m}$ of positive resist (OIR 907/17, Fujifilm) is spun on a $500 \mu \mathrm{m}$ borofloat wafer followed by exposure and development for structure definition. The electrodes are deposited by 
sputtering a $25 \mathrm{~nm}$ titanium adhesion layer and a $500 \mathrm{~nm}$ gold layer. Excess metal is removed by lift-off in aceton. Afterwards, the wafer is diced into individual chips of $2 \times 5 \mathrm{~mm}$. For convenient handling the chips are glued to a printed circuit board (PCB) using Loctite M-31CL Hysol. Electrical connections from the PCB to the IDA electrode on the chip are made using a wirebonder (Westbond). Finally, an additional layer of Hysol is added to shield the contact pads and wirebonds from the solution.

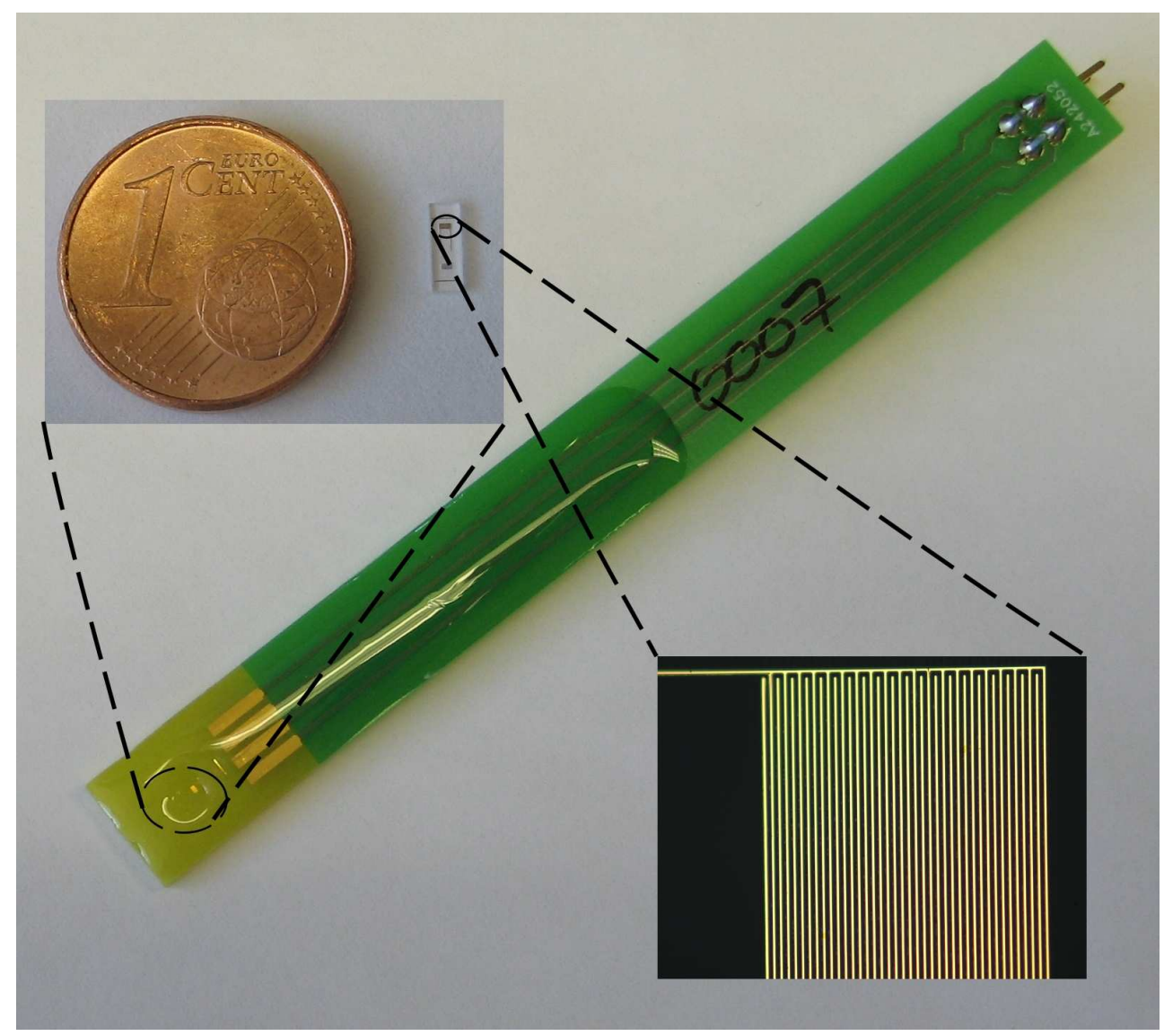

Figure 6.4: Photographs of the sensor glued on a PCB for easy handling (middle), and next to one euro cent (upper-left inset). Microscope image of the IDA electrode (lower-right inset). each individual finger is $557 \mu \mathrm{m} \times 4 \mu \mathrm{m}(\mathrm{l} \times \mathrm{w})$ with a 4 um gap. The total IDA contains 20 finger pairs.

The resulting sensor is depicted in figure 6.4. In the middle the entire sensor assembly is visible, in the upper left corner the individual chip and on the lower right corner a microscope image of the IDA electrode. Each individual finger is $557 \mu \mathrm{m}$ long and $4 \mu \mathrm{m}$ wide, with a $4 \mu \mathrm{m}$ gap in between. The total electrode height is $525 \mathrm{~nm}$, and the 
IDA contains 20 finger pairs.

\subsubsection{Chemicals}

A solution of $4 \mathrm{mM}\left[\mathrm{Ru}\left(\mathrm{NH}_{3}\right)_{6}\right] \mathrm{Cl}_{3}$ and $1 \mathrm{mM}$ catechol in $100 \mathrm{mM}$ phosphate buffer ( $\mathrm{KH}_{2} \mathrm{PO}_{4} / \mathrm{K}_{2} \mathrm{HPO}_{4}$, pH 7) is used for electrochemical measurements. The solution is purged with argon gas for at least $15 \mathrm{~min}$ prior to the DCV measurement, and additionally kept under argon atmosphere during experiments. All chemicals are obtained from Sigma-Aldrich.

\subsubsection{Methods}

All potentials reported here are measured versus an $\mathrm{Ag} / \mathrm{AgCl}$ (saturated $\mathrm{KCl}$ ) reference electrode (Radiometer Analytical), and a platinum counter electrode is utilized. For electrochemical measurements a bipotentiostat is used (bistat, Bio-Logic SAS). Each channel is programmed using a conventional CV technique with a fixed potential offset between the two channels. Both channels are started synchronously using standard options in the control software of the bipotentiostat.

\subsection{Results and Discussion}

\subsubsection{Simulation results}

\subsubsection{SIBB condition validation}

In the previous section we reported the use of a semi-infinite bulk boundary (SIBB) condition at a parallel plane to and far away from the substrate. A small study has been conducted in which the distance of the SIBB and the simulation time is varied while maintaining the rest of the geometry equal to the configuration of figure $6.2 \mathrm{a}$ with gap and electrode widths of $2 \mu \mathrm{m}$. The results are shown in figure 6.5. The percentages in the figure are calculated by dividing the total flux of ions of one species at the SIBB with the average flux of ions of that same species at the generator electrode, which is estimated as $2 \cdot 10^{-9} \mathrm{~mol} \cdot \mathrm{m}^{-1} \cdot \mathrm{s}^{-1}$. As stated before, a two dimensional model is used. Therefore the flux $(\mathrm{N})$ has dimensions $\mathrm{mol} \cdot \mathrm{m}^{-1} \cdot \mathrm{s}^{-1}$.

We see five lines representing the results from five simulations with different positions for the SIBB layer. As expected, the ions reach the SIBB faster if the SIBB is placed closer to the electrodes. The line corresponding to a position of $y=10 \mu \mathrm{m}$ for the SIBB shows an area at which the flux is not changing any more for increasing time. This might be explained by the observation that an equilibrium is reached 


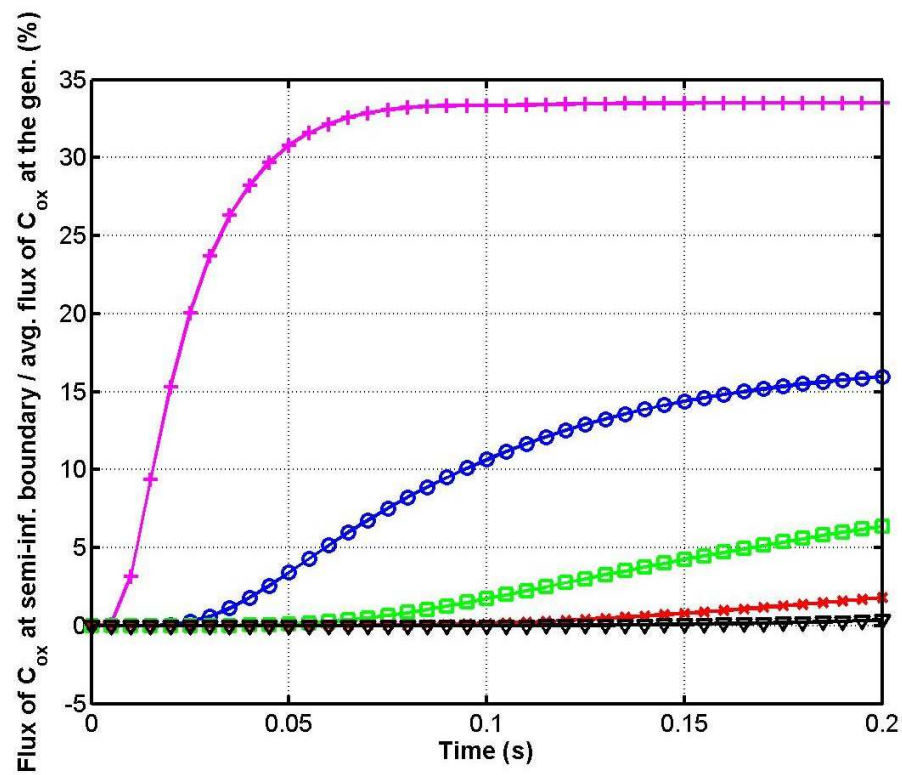

Figure 6.5: Flux of $C_{o x 1}$ at the semi-infinite boundary divided by the estimated flux at the generator electrode $\left(2 \cdot 10^{-9} \mathrm{~mol} \cdot \mathrm{m}^{-1} \mathrm{~s}^{-1}\right)(\%)$ vs. time for $y=10 \mu \mathrm{m}[+]$, $y=20 \mu \mathrm{m}[\mathrm{o}], y=30 \mu \mathrm{m}[\boxminus], y=40 \mu \mathrm{m}[\mathrm{x}]$ and $y=50 \mu \mathrm{m}[\nabla]$

at which a fully developed concentration profile is seen between the SIBB and the electrodes. For larger distances between the electrodes and the SIBB the flux at the SIBB is negligible compared to the flux at the generator electrode.

From this study it can be concluded that it is important to choose the right semiinfinite boundary distance in combination with the right simulation time. In further simulations a distance of $50 \mu \mathrm{m}$ is used in combination with a simulation time of $0.1 \mathrm{~s}$ unless mentioned otherwise. The current of both the generator and collector electrode is already (quasi) steady-state at this time, while the flux of ions at the semi-infinite boundary is still zero.

\subsubsection{Electrode size study}

From previous simulations shown in literature $[4,12]$ it is known that the collection efficiency will increase with decreasing gap size between the generator and collector electrode. To confirm the validity of our model, the geometry shown in figure $6.2 \mathrm{~A}$ is simulated with different gap and electrode widths. The results are shown in figure 6.6. The electrode size and gap width was decreased down to $30 \mathrm{~nm}$. Since double layers at the electrode surfaces have a typical thickness up to a few nanometers with 
a strong electrolyte present, migration might start to play a significant role at gap widths smaller than $30 \mathrm{~nm}$.

In figure 6.6a four lines representing the collection efficiency are visible, each with a different ratio of $w_{e} / w_{g}$. It is interesting to notice that the differences between these lines are small. Apparently the gap width is a more important factor determining the collection efficiency than the electrode width.

From figure 6.6a it can be concluded that the collection efficiency is increasing almost linearly with decreasing gap width. The results found at $W_{g}=1 \mu \mathrm{m}$ and $W_{e}=1 \mu m$ from this simulation (87.4\%) are comparable with the simulated results from Bard [4] (85\%) and Yang [12] (87\%). The small deviations between these simulations might be explained because of small differences between the choice for the position of the semi-infinite bulk boundary condition $(50 \mu \mathrm{m})$, the choice for the simulation time $(0.1 \mathrm{~s})$, the choice for parameters like the diffusion constant and bulk concentration and the number of mesh elements.

Figure 6.6b shows the number of redox cycles as calculated from the collection efficiencies presented here. In figure 6.6b, five points are illustrated in red which correspond to the maximum number of redox cycles achieved in experiments by the groups of Ueno et al. [17], (2) Paeschke et al. [11], (3) Bard et al. [4], (4) Niwa et al. [9] and (5) V.A.T. Dam [21]. Point 2 and 3 seems to be corresponding with our simulation results. However, the last two points $(4,5)$ from the group of Niwa et al. and V.A.T. Dam report a redox cycling amplification which is higher than expected, while the redox cycling achieved by the group of Ueno is lower than the results calculated from this simulation. This unexpected behavior of the sensor of V.A.T. Dam is investigated in the next section. 


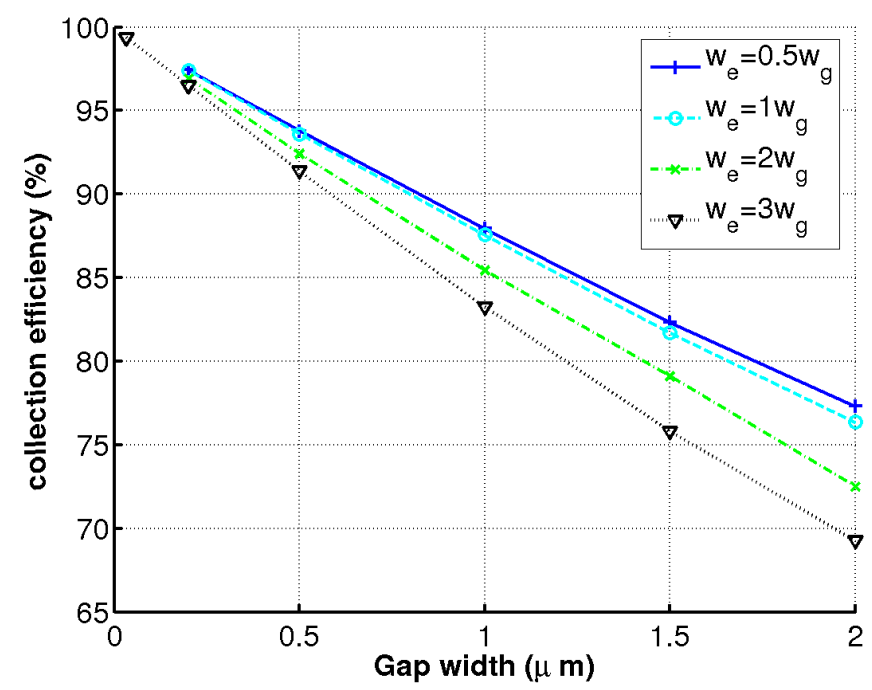

(a) Collection efficiency (\%)

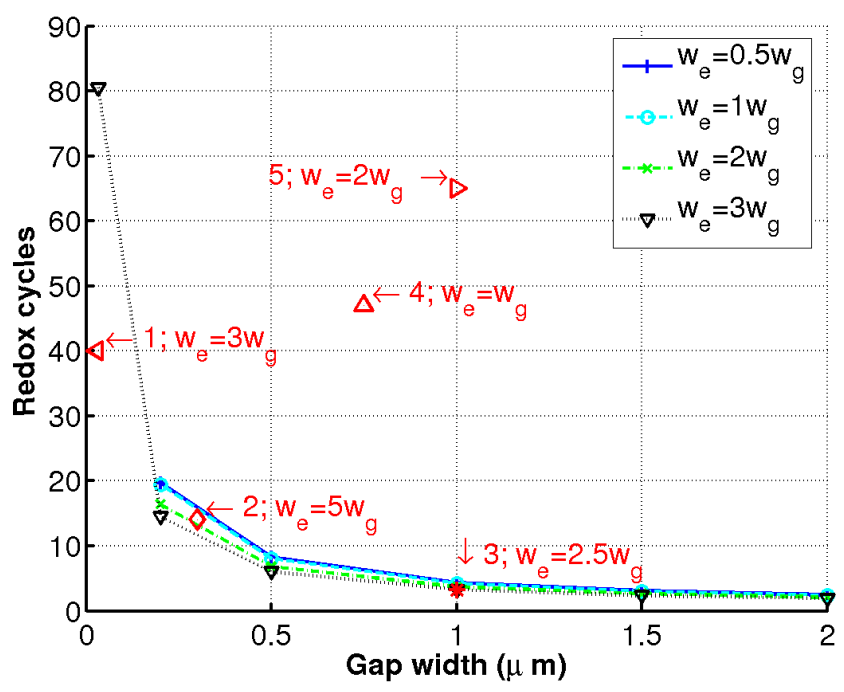

(b) Redox cycles

Figure 6.6: Collection efficiency (a) and redox cycles (b) for IDA electrodes for different gap and electrode widths. The red points represent the maximum achieved redox cyles as reported by (1) Ueno et al. [17], (2) Paeschke et al. [11], (3) Bard et al. [4], (4) Niwa et al. [9] and (5) V.A.T. Dam [21]. 


\subsubsection{Electrode height study}

We have studied the influence of the electrode height $(h)$ on the collection efficiency. The results are shown in figure 6.7. The geometry used, is shown in figure 6.2C. The dimensions are: $W_{g, t}=W_{g, b}=W_{e}=2 \mu m$ for the red line, and $W_{g, t}=W_{g, b}=$ $W_{e}=1 \mu \mathrm{m}$ for the blue line. The electrode height is varied between $100 \mathrm{~nm}$ and $7 \mu m$.

There is clearly an increase in collection efficiency and redox cycling because the generated species are partly enclosed in the trench formed by the space between the elevated electrodes. The increase in collection efficiency leads to an increased number of redox cycles, as is shown in figure 6.7b. Apparently, the number of redox cycles has a linear relation to the electrode height.

Figure $6.2 \mathrm{~b}$ shows a SEM-picture of the device used by V.A.T. Dam [21]. By using the scale printed in the figure, parameters of the geometry are estimated as $w_{g, t}=1 \mu m, w_{g, b}=0.5 \mu m$ and $w_{e}=2 \mu m$. The trench is formed using deep reactive ion etching. Its depth is approximately $7 \mu \mathrm{m}$ according to the original paper. The metal of the electrodes is added by sputtering the trench under an angle of $45^{\circ}$. It is therefore likely that the trench in the experimental setup was somewhat tapered leading to a smaller gap width at the bottom of the trench.

A simulation has been conducted using this geometry. The results show a generator current of $3.507 \mathrm{E}-3 \mathrm{~A} / \mathrm{m}$ and a collector current of $3.483 \mathrm{E}-3 \mathrm{~A} / \mathrm{m}$ leading to a collection efficiency of $99.3 \%$. This is equivalent to a redox cycling number of approximately 74 times. This result is very close to the 60 to 70 times found by V.A.T. Dam. Apparently, the increased electrode height of the electrodes result in the high redox cycling amplification we observed in these experiments.

\subsubsection{Selectivity study using a fixed generator potential}

The redox cycling effect can be used to accomplish selective detection among a selected set of redox active species. We have extended our simulation model to simulate cyclic voltammetry measurements. Since the simulated experiment time is increased, it was necessary to place the SIBB-layer at a distance of 4E-4 $\mathrm{m}$ away from the electrodes. For this simulation the geometry used is shown in figure 6.2a with $w_{e}=w_{g}=2 \mu \mathrm{m}$. If a potential sweep of $50 \mathrm{mV} / \mathrm{s}$ is applied to both generator and collector electrode simultaneously, the result shown in figure $6.8 \mathrm{a}$ is observed. The lines represent the current from the ferro/ferricyanide redox couple (red), the dopamine couple (green) and the total current (blue). 


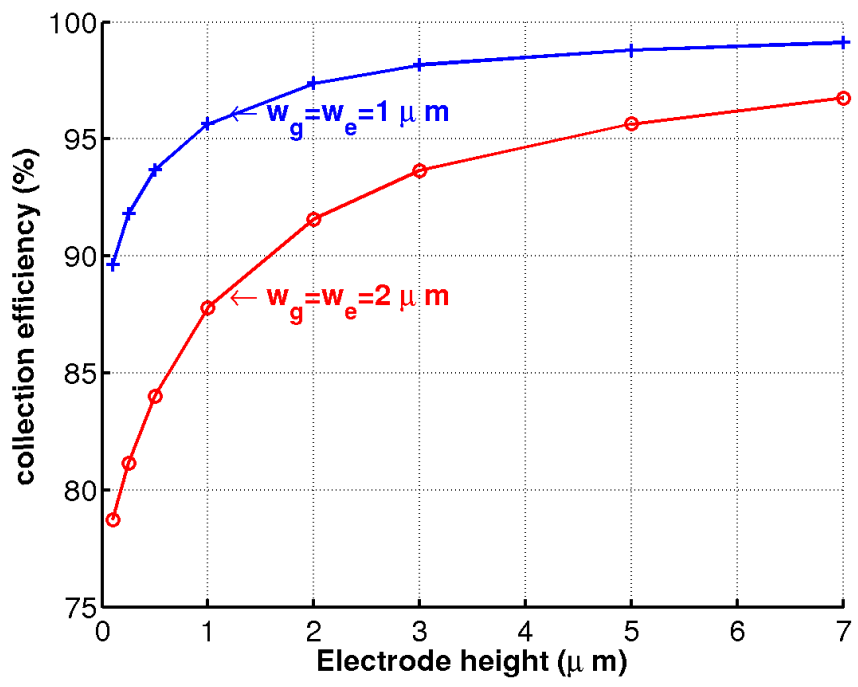

(a) Collection efficiency (\%)

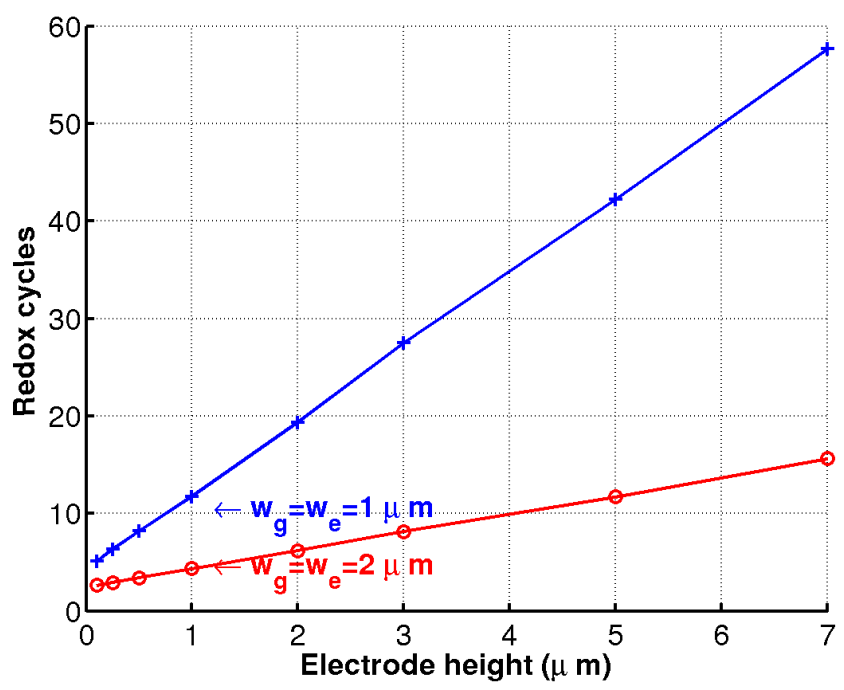

(b) Redox cycles

Figure 6.7: Collection efficiency (a) and redox cycles (b) for IDA-electrodes with raised electrodes.

In figure $6.8 \mathrm{~b}$ the result is shown for a single potential sweep applied to the collector electrode, while the potential of the generator electrode is fixed at $0.4 \mathrm{~V}$. For the 
first redox couple with a standard potential of $0.265 \mathrm{~V}$, the generator potential can be regarded as an oxidating potential. However, for the second redox couple with a standard potential of $0.5 \mathrm{~V}$, the generator potential is a reducing potential. Therefore redox cycling will occur for the first redox couple if the collector potential is below $0.265 \mathrm{~V}$, while redox cycling for the second couple will occur if the collector potential is above $0.5 \mathrm{~V}$. In figure $6.8 \mathrm{~b}$, we see that the current of species 1 is indeed clearly increasing for collector potentials below $0.265 \mathrm{~V}$, while the current of species 2 is amplified at collector potentials above $0.5 \mathrm{~V}$. The selectivity achieved for the ferrocyanide/ferricyanide redox couple at a collector potential of $-0.1 \mathrm{~V}$ is $i_{s p 1} / i_{s p 2}=11.0$, while the selectivity achieved for the dopamine redox couple at a collector potential of $0.9 \mathrm{~V}$ is $i_{s p 2} / i_{s p 1}=10.2$. From this simulation it can be concluded that the redox cycling effect can be used to obtain selective detection in a set of redox couples with different standard potentials.

\subsubsection{Differential cyclic voltammetry study}

The simulated results of the DCV study are shown in figure 6.9a. For this simulation the geometry used is shown in figure 6.2a with $w_{e}=w_{g}=4 \mu \mathrm{m}$, to match the dimensions of the fabricated sensor shown in figure 6.4. The current contribution of the third redox couple $\left(\mathrm{sp} 3, E^{0}=-0.16 \mathrm{~V}, C^{*}=4 \mathrm{mM}\right)$ is indicated in red, and the current contribution of the fourth redox couple $\left(\mathrm{sp} 4, E^{0}=0.2 \mathrm{~V}, C^{*}=1 \mathrm{mM}\right)$ in green. The total current is illustrated in blue. Two peaks are clearly visible at the position of the two standard potentials of both redox couples. The peak currents of the third and fourth couple are $100 \mu \mathrm{A} / \mathrm{m}$ and $41 \mu \mathrm{A} / \mathrm{m}$ respectively, thus the ratio between both peak currents is 2.4 .

The expected peak ratio is 2 since catechol has a 4 times lower concentration but a two times higher contribution to the current due to a two-electron transfer. The slightly different ratio is caused by the difference in rate constants between couple 3 and 4 . The rate constants used for the simulations are aimed to be close to the actual rate constants of $\mathrm{Ru}\left(\mathrm{NH}_{3}\right)_{6}$ and catechol, which differ approximately by one order of magnitude as indicated in table 6.1.

\subsubsection{Experimental results}

In figure $6.9 \mathrm{~b}$ the experimental result of a DCV measurement is shown. In this measurement two peaks can be observed at $-0.17 \mathrm{~V}$ and $0.19 \mathrm{~V}$ corresponding to the standard potentials of $\left[\mathrm{Ru}\left(\mathrm{NH}_{3}\right)_{6}\right] \mathrm{Cl}_{3}$ and catechol, respectively [20,31]. The peak height is determined by compensating for the baseline using the red line illustrated in figure 5. Using this compensation the peak heights are determined to be $2.29 \mu \mathrm{A}$ and $0.54 \mu \mathrm{A}$ for the left and right peak, respectively. Therefore, the ratio between the peaks is 4.2 . 


\subsubsection{Model and experimental agreement}

Comparing the results from simulated and experimental data we observe that the ratio of peak heights is slightly different in both cases. We believe this to be caused by adsorption of catechol to the gold surface during the experiments. Also, if the results from the theoretical model are multiplied with the length and amount of finger pairs of the IDA electrode, the resulting peak heights become $2.23 \mu \mathrm{A}$ and $0.91 \mu \mathrm{A}$. The value of the $\mathrm{Ru}\left(\mathrm{NH}_{3}\right)_{6}$ is in good agreement with experimentally obtained values. 


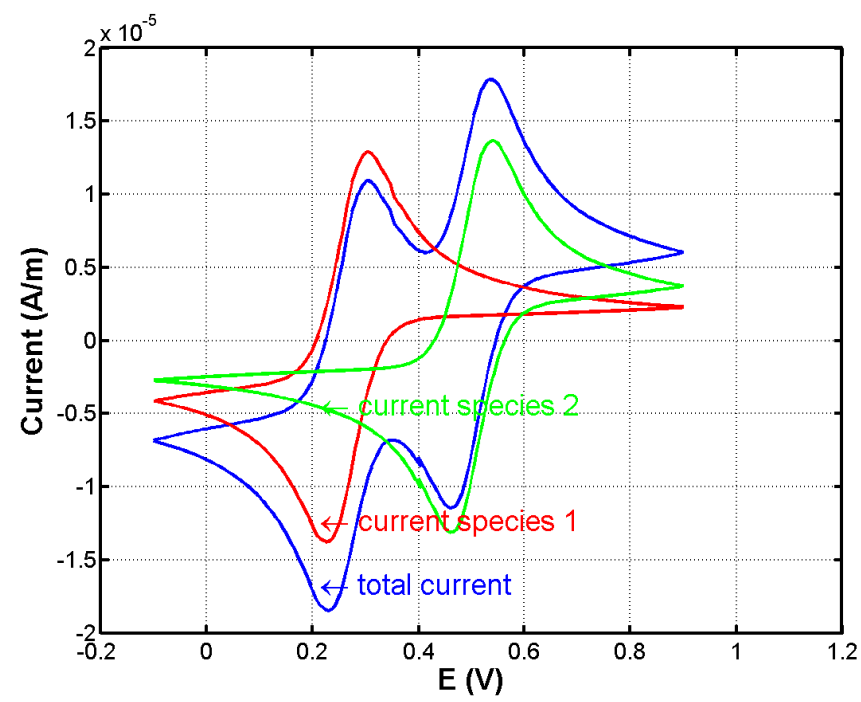

(a) Cyclic voltammogram - simultaneous sweep applied to collector and generator electrode.

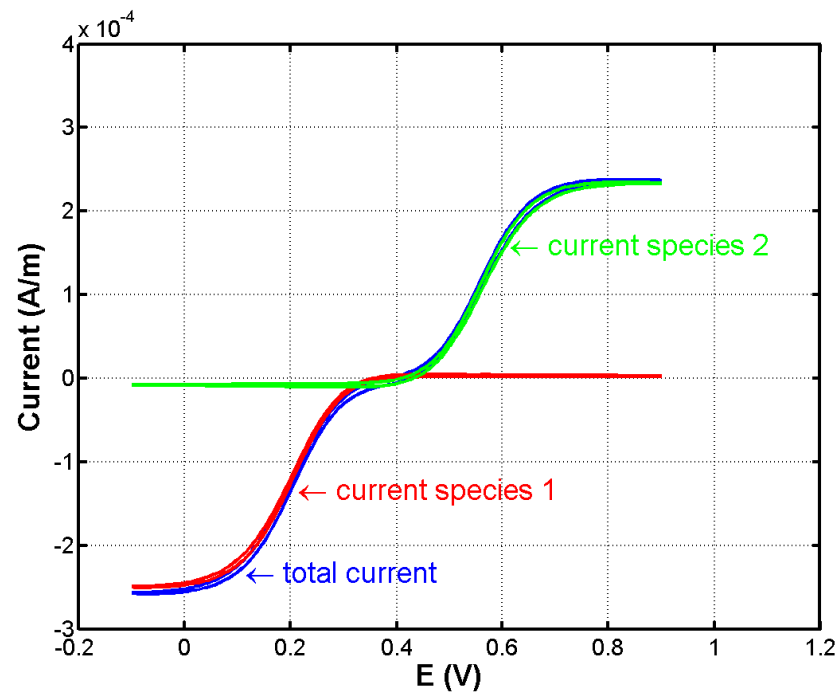

(b) Cyclic voltammogram - single sweep applied to collector electrode, while $\mathrm{Eg}=0.4 \mathrm{~V}$.

Figure 6.8: Selectivity simulations. Red: Current species 1 - the ferrocyanidelferricyanide redox couple. Green: Current species 2 - the dopamine redox couple. Blue: Total current. 


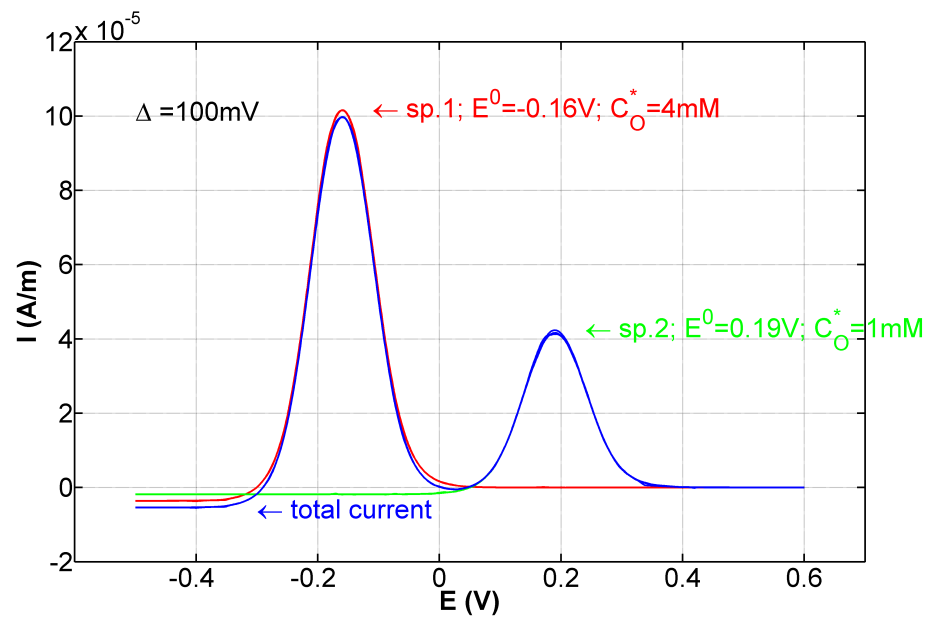

(a) Simulation result of a DCV measurement

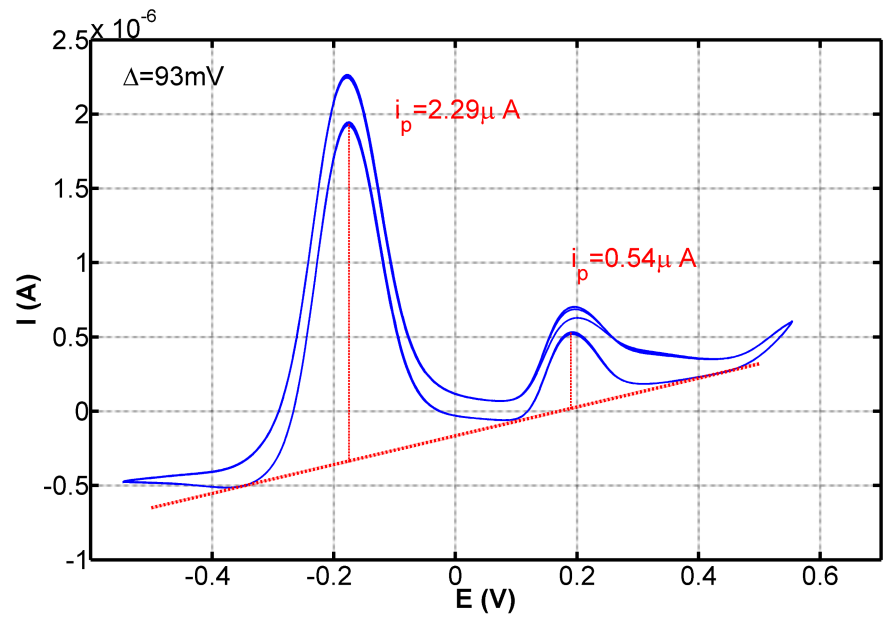

(b) Experimental result of a DCV measurement using the sensor shown in figure 6.4. Potential measured vs. $\mathrm{Ag} / \mathrm{AgCl}$, using a supporting electrolyte of $100 \mathrm{mM}$ phosphate buffer at $\mathrm{pH} 7$.

Figure 6.9: Simulation and experimental DCV results using a scan rate of $50 \mathrm{mV} / \mathrm{s}$ and an potential difference of $100 \mathrm{mV}$. Two reversible redox couples are used; $\mathrm{Ru}\left(\mathrm{NH}_{3}\right)_{6}$ (Sp.3. $\left.\mathrm{C}^{*}=4 \mathrm{mM}, \mathrm{E}^{0}=-0.16 \mathrm{~V}\right)$ indicated in red and catechol (Sp.4. $\left.C^{*}=1 \mathrm{mM}, E^{0}=0.2 \mathrm{~V}\right)$ indicated in green. Blue lines in both subfigures indicate the total (measured) current. 


\subsection{Conclusion}

In this chapter simulations on the interdigitated array electrode have been presented. A method is developed to ensure that results from the simulations correspond with the associated real-world experiments they represent. Within this method suitable domain equations, boundary conditions and guidelines for simulation time have been formulated. This method is tested with the IDA-geometry with flat electrodes to check its validity. The results in terms of collection efficiency for a $w_{e}=w_{g}=1 \mu \mathrm{m}$ structure presented in this report (87.4\%) are comparable with results from a paper of Yang and Zhang [12] (87\%) and a paper of Bard et al. [4] (85\%).

Simulations on IDA-electrodes in which the electrode height is varied, showed an increase in redox cycling for raised electrodes. The special case with an estimated geometry derived from the devices used by V.A.T. Dam [21] is simulated. The result shows $74 \mathrm{x}$ redox cycling, which is very close to the 60 to 70 times found by V.A.T. Dam. Therefore a plausible explanation is given for the unusually and unexpectedly high redox cycling found in the experimental results of V.A.T. Dam.

We have also shown that the redox cycling can be used as a tool to achieve a selective measurement in a mixture of multiple redox couples using a cycling potential at one electrode and a fixed potential at the other electrode. We have achieved a selectivity of $11 \mathrm{x}$ in a mixture of the ferrocyanide/ferricyanide and dopamine redox couple with an IDA electrode with geometry $w_{e}=w_{g}=2 \mu \mathrm{m}$.

Finally, we propose a novel electrochemical measurement technique based on redox cycling, which can be used for selective and simultaneous measurements in mixtures of multiple reversible redox couples. This technique which we have named DCV is based on recording two cyclic voltammograms with a small potential offset using IDA electrodes. Current amplification only occurs if one electrode is at a reducing potential while the other electrode is at an oxidizing potential for a specific reversible redox couple. As such, a strong increase in current is only observed if the potentials of both electrodes are surrounding the standard potential of a reversible redox couple. Therefore, the obtained data is comparable to the results obtained with differential pulse voltammetry (DPV). Compared to DPV, DCV has the added benefit of more selectivity even in the presence of high amounts of interfering non-reversible redox active species. Using DCV we have determined both theoretically and experimentally standard potentials and concentrations of a mixture of $4 \mathrm{mM}\left[\mathrm{Ru}\left(\mathrm{NH}_{3}\right)_{6}\right] \mathrm{Cl}_{3}$ and $1 \mathrm{mM}$ catechol simultaneously.

Future work will be focused towards sensors showing higher redox cycling amplification and measurements in solutions containing high concentrations of interfering compounds like ascorbic acid. 


\section{Bibliography}

[1] M. Odijk, W. Olthuis, V. Dam, and A. van den Berg. Electroanalysis, 20(5):463-468, 2008.

[2] F.-R. F. Fan and A. J. Bard. Science, 267(5199):871-874, 1995.

[3] D. G. Sanderson and L. B. Anderson. Anal Chem, 57:2388-2393, 1985.

[4] A. J. Bard, J. A. Crayston, G. P. Kittlesen, T. V. Shea, and M. S. Wrighton. Anal Chem, 58:2321-2331, 1986.

[5] B. Seddon, H. Girault, and M. Eddowes. J Electroanal Chem, 266:227-238, 1989.

[6] Y. Iwasaki and M. Morita. Current separations, 14:1-8, 1995.

[7] P. Jin, A. Yamaguchi, F. A. Oi, shigeki Matsuo, J. Tan, and H. Misawa. Analytical sciences, 17:841-846, 2001.

[8] J. Min and A. J. Baeumner. Electroanalysis, 16(9):724-729, 2004.

[9] O. Niwa, M. Morita, and H. Tabei. Anal Chem, 62:447-452, 1990.

[10] O. Niwa, H. Tabei, B. P. Solomon, F. Xie, and P. T. Kissinger. Journal of Chromatography B, 1:21-28, 1995.

[11] M. Paeschke, U. Wollenberger, C. Kohler, T. Lisec, U. Schnakenberg, and R. Hintsche. Analytica Chimica Acta, 305:126-136, 1995.

[12] X. Yang and G. Zhang. Comsol Proceedings and user presentations CD, 1:1-6, 2005.

[13] F. Bjorefors, C. Strandman, and L. Nyholm. Electroanalysis, 12-4:255-261, 2000.

[14] K. Hayashi, Y. Iwasaki, R. Kurita, K. Sunagawa, O. Niwa, and A. Tate. J Electroanal Chem, 579:215-222, 2005.

[15] R. Kurita, H. Tabei, Z. Liu, T. Horiuchi, and O. Niwa. Sensors and actuators $B, 71: 82-89,2000$.

[16] H. Tabei, M. Takahashi, S. Hoshino, O. Niwa, and T. Horiuchi. Anal Chem, 66(20):3500-3502, 1994.

[17] K. Ueno, M. Hayashida, J.-Y. Ye, and H. Misawa. Electrochemistry Communications, 7(2):161-165, 2005.

[18] E. D. Goluch, B. Wolfrum, P. S. Singh, M. A. G. Zevenbergen, and S. G. Lemay. Anal Chem, 394:447-456, 2009.

[19] M. Zevenbergen, D. Krapf, M. Zuiddam, and S. Lemay. Nano Lett, 7(2):384388, 2007.

[20] B. Wolfrum, M. Zevenbergen, and S. Lemay. Anal Chem, 80:972-977, 2008.

[21] V. A. T. Dam, W. Olthuis, and A. van den Berg. The Analyst, 1:1, 2007. doi: 10.1039/b616667a. DOI: 10.1039/b616667a.

[22] J. Strutwolf and D. E. Williams. Electroanalysis, 17:169-177, 2004. 
[23] K. Ueno, H. Kim, and N. Kitamura. Anal Chem, 75(9):2086-2091, 2003.

[24] C. Gabrielli, M. Keddam, P. Rousseau, and V. Vivier. Comsol Proceedings and user presentations $C D$, 1:1-5, 2005.

[25] A. J. Bard and L. R. Faulkner. Electrochemical methods - fundamentals and applications. Wiley, 2nd edition, 2001.

[26] J. Wiedemair, N. Menegazzo, J. Pikarsky, K. S. Booksh, B. Mizaikoff, and C. Kranz. Electrochimica Acta, 55:5725-5732, 2010.

[27] R. de Cassia Silva Luz, F. S. Damos, A. B. de Oliveira, J. Beck, and L. T. Kubota. Sensors and Actuators B, 117:274-281, 2006.

[28] M. R. Deakin, P. M. Kovach, K. J. Stutts, and R. M. Wightman. Anal Chem, 58:1474-1480, 1986.

[29] H. Deng and G. J. V. Berkel. Electroanalysis, 11(12):857-865, 1999.

[30] D. W. M. Arrigan, M. Ghitab, and V. Benia. Chem Commun, 0:732?733, 2004.

[31] D. E. Khoshtariya, T. D. Dolidze, A. Vertova, M. Longhi, and S. Rondinini. Electrochistry Communications, 5:241-245, 2003. 


\section{Conclusions and outlook}

In this chapter, a short summary will be presented of the main results and conclusions presented in the previous chapters. Moreover, an outlook to future perspectives of this work will be discussed.

\subsection{Conclusions}

Chapter two establishes the need for a miniaturized electrochemical cell in the field of drug screening and electrochemical protein cleavage. This miniaturized cell requires a special design to be able to comply with the requirements for these applications. Design demands include a high conversion efficiency, resistance to commonly used solvents while maintaining a small internal volume.

Such a miniaturized cell design is presented in chapter three. The demonstrated cell is composed of a glass microfluidic chip, platinum work and counter electrodes and a palladium pseudo-reference electrode. This chip is characterized electrochemically by CV measurements under flow conditions. Also, a conversion efficiency of $97 \%$ is demonstrated using a fixed applied potential for the small and fast-reacting ferro/ferricyanide redox couple at a rate of $1 \mu \mathrm{L} / \mathrm{min}$ using UV-vis spectroscopy as off-chip detection method. Moreover, this chip is shown to be feasible for use in drug screening studies. This is demonstrated by direct electrochemical generation of the main metabolic products of amodiaquine, a known antimalarial agent. The chip shows similar performance to commercially available flow-through cells while requiring less analyte.

In chapter four, an improved design of this miniaturized electrochemical cell is presented. The first prototype showed some issues with the palladium pseudo-reference electrode. We believe these issues to be caused by intrinsic stress in the thin-film electrode by volume changes related to hydrogen uptake. The improved cell design incorporates an iridium oxide (IrOx) pseudo-reference electrode. The IrOx pseudoreference shows a drift of max. $5 \mathrm{mV}$ per 20 minutes, which corresponds to the time 
required for a typical conversion experiment. The improved chip is used to study the oxidative metabolism of procainamide, a known antiarrythmic drug. The traditional method using rat and human liver cell microsomal incubation only gives information on two out of four known metabolites. The chip however is able to generate all four main metabolites, which indicates that direct oxidation is definitely useful as complementary method in the pre-clinical phase of drug development.

Chapter four also demonstrates a new measurement setup. As the microfluidic chip shows near total conversion efficiency for small and fast reacting ions, it is unintentionally a very sensitive electrochemical flow meter. A change in flow rate of inwards electrolyte will induce a change of introduced ions, resulting in a change in measured current during a typical conversion experiment. This observation implies the need for very precise pumps and a well defined microfluidic environment surrounding the microfluidic chip. This resulted in the development of the Lab-in-a-Suitcase, which is basically a portable setup containing precise syringe pumps, low-internal volume fluidic valves and a portable potentiostat. Total internal volume of all microfluidic tubing and connections is estimated to be below $5 \mu L$, while continuous flow rates down to a few tens of nanoliters/min can be applied.

Chapter five demonstrates that electrochemical cleavage of proteins is feasible using the same improved cell design by some preliminary results. Some electrochemical cleavage is observed for the tripeptide leucine - tyrosine - leucine. However, overall oxidation and cleavage yields were low. A new cell design specifically aimed for this purpose is required to make the most out of this application. Moreover, surrounding equipment like the mass spectrometer and the microfluidic chip need to be matched in terms of flow rates and sensitivity to improve future results.

In chapter six, the phenomenon of redox cycling at facing interdigitated array electrodes is used to demonstrate a novel measurement method called differential cyclic voltammetry (DCV). Using DCV, it was shown both theoretically and experimentally that both catechol and ruthenium - hexaammine - trichloride could be detected simultaneously and selectively.

\subsection{Outlook}

One of the critical remarks once heard during a conference on analytical chemistry is that the microfluidic community can fabricate really impressive devices in terms of performance, but looses interest once a proof of concept is ready. Often, microfluidic devices require a trained microfluidics expert to be operated. Also, microfluidic devices are not always as reliable as required by the analytical chemistry community. 
This community is often quite conservative when it comes to the application of labon-chip technology. This seems only logical since the chemical challenges faced are often difficult enough without the added complexity of more unreliable results from microfluidic devices.

Therefore one of the questions that can be asked at the end of this project, is whether the work presented in this thesis is ready for routine use in the previously discussed areas. Significant progress is made to achieve that point. The Lab-in-a-Suitcase and the improved microfluidic chip presented in chapter 4 demonstrates continuous, reliable operation. Also, the setup and automated software developed for the Labin-a-Suitcase do no longer require a well trained microfluidics expert to be operated. However, few challenges still remain.

In the work presented in chapters 4,5 and 6 , the generated products of the microfluidic chip are often collected in a sample loop before analysis by the mass spectrometer. This loop requires extensive filling times, especially at low flow rates. Therefore the time required for a single measurement is quite large. Obviously, studies of short-living compounds is not possible using this approach. Future work might be focused towards the development of an integrated electrospray ionization (ESI) needle onto the chip. The integration of an ESI needle offers several improvements. Dead volumes between the active area of the chip and the MS orifice is greatly reduced. Thereby also reducing the time between on-chip product generation and analysis in the MS down to the subsecond regime, if designed properly.

The results shown in this thesis use platinum as working electrode material. Platinum is an electrode material that can be deposited reliably and with ease on glass substrates. However, for some applications other electrode materials might be more suited from an electrochemical point of view. Carbon or diamond based electrodes show a larger potential window in aqueous media. Also, doped diamond provides the possibility to generate hydroxyl radicals. One of the challenges that remain for future research is to incorporate these electrode materials into a microfluidic chip. Especially doped diamond is difficult to fabricate and not compatible with the fabrication methods used in this thesis.

Lab-on-chip technology provides the promise of disposable devices, circumventing the need of extensive cleaning protocols of the electrode surface. Disposable chips using the same fabrication platform have been demonstrated by the company Medimate [1]. However, the time, money and effort required to fabricate the currently presented chip is quite large if fabricated in small quantities. Future efforts towards the use of an even cheaper fabrication method might be beneficial in several aspects. First, it might be difficult to include an on-chip ESI needle using the current 
glass substrate. Switching to a different substrate material like cyclic olefin copolymers (COC) might reduce costs, while providing opportunities for easy ESI needle fabrication. Moreover, LC columns are shown to be fabricated by nanoimprint techniques using $\mathrm{COC}$ as substrate material [2]. An integrated device composed of an electrochemical converter, LC column separator and efficient electrospray ionization interface seems to be in reach for a future project.

\section{Bibliography}

[1] A. Floris, S. Staal, S. Lenk, E. Staijen, D. Kohlheyer, J. Eijkel, and A. van den Berg. Lab on Chip, 10:1799-1806, 2010.

[2] X. Illa, W. D. Malsche, J. Bomer, H. Gardeniers, J. Eijkel, J. Morante, A. Rodriguez, and G. Desmet. Lab on Chip, 9:1511-1516, 2009. 


\section{Appendix}

This appendix shows the cleanroom process document and the fabrication details of the IrOx in particular. 


\section{A.1 Process document for the EC cell on-chip}

\section{A.1.1 Introduction}

To the best of my knowledge this is the fifth revision of the picoAspect and LC-ECMS process flow. The first author is Dorothee(?) Hermes. I've adopted this process flow for the LC-EC-MS project and made mainly changes to the layout of channel and size of counter electrode. The powderblast holes should fit on a normal (not the special one with abnormal positioning of the probe pins) picoAspect holder as well as the new design made in 2007-2009 with the mmcx coaxial connectors or the modular PCB layout. This version contains all the current measurement data (like sputter-rates and etch rates) known at the moment of this writing (Sept. 2010). I believe they are quite accurate, although these data are still measured in the old MESA+ cleanroom.

The new parameters are derived from the following paper: S. Negi, R. Bhandari, L. Rieth and F. Solzbacher, Effect of sputtering pressure on pulsed-DC sputtered iridium oxide films. Sensors and Actuators B, Nov. 2008. A second issue observed with the V2 chips are the compliance errors from the potentiostat. This is solved by a better position of the reference electrode in between the work and counter electrode.

People involved with this project are/were Jan van Nieuwkasteele, Roald Tiggelaar, Johan Bomer, Paul ter Braak, Monica Brevio and myself.

\section{A.1.2 Mask layout}

\section{A.1.2.1 Total design}

In total 7 layers are included in this design. Some of these layers are mutually exclusive to realize different work electrode materials. A standard set of masks used to process the bottom wafer to complete chips with platinum work and counter electrodes and an IrOx reference electrode placed on the right side of the work electrode looks like this:

Mask 2b, inside white, labelled: All Pt.

Mask 3c, inside white, Labelled: Ref. El. (there are two of those, beware!) 


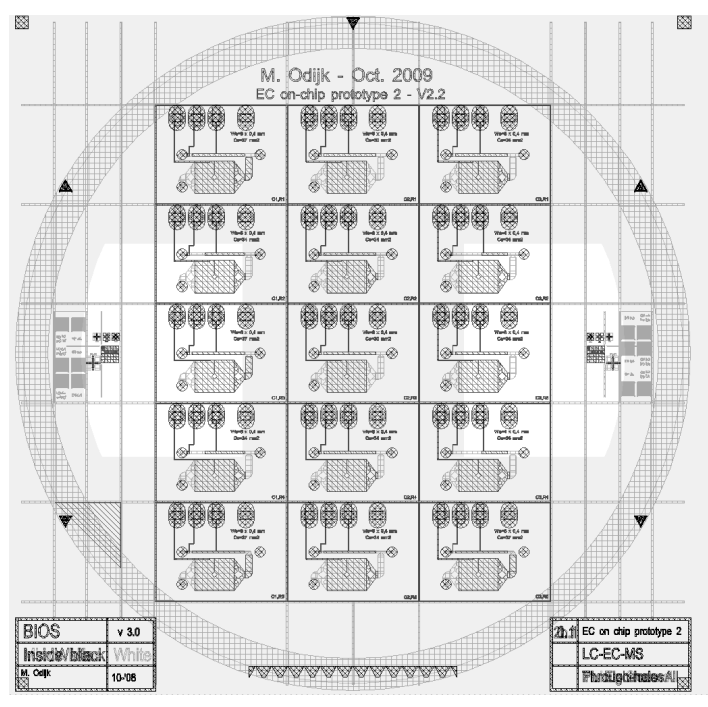

Figure A.1: Overview of the total wafer including all wafers.

For all possible variations, the process of the top wafer remains unchanged. Therefore the following masks are used to process the top wafer:

Mask 1, inside white mirrored, labelled: fluidic channel.

Mask 0, inside black, labelled: through holes (used for powderblasting).

The remaining masks can be used to vary the work electrode material. To do so, use the following masks in the order below: Mask 2a, inside white, labelled: pt electrode.

Mask 4, inside white, labelled: work electrode.

Then continue like normal with mask 3c, inside white, Labelled: Ref. El.

Finally there is one mask with number 3 labelled ref. el. which can be used to make an IrOx reference electrode on the 'old' position on the left side of the work electrode. This mask was replaced by mask $3 b$ with the label IrOx fix, and then replaced again by mask $3 \mathrm{c}$ labelled Ref. El.. The main difference between $3 \mathrm{~b}$ and $3 c$ is that in $3 b$, only the area in contact with the fluid gets a cover by an IrOx layer, while in $3 \mathrm{c}$ the IrOx is also present at the contact pads. The reason for this difference is that $3 \mathrm{c}$ is used in conjunction with TCoater, where sputtering of Ti/Pt/Ti/IrOx layer in a single run is possible. The single run is preferrable, since otherwise adhesion between $\mathrm{Ti}$ and $\mathrm{IrOx}$ is not good due to oxidation of the Ti layer by the atmosphere. 


\section{A.1.2.2 Chip designs}

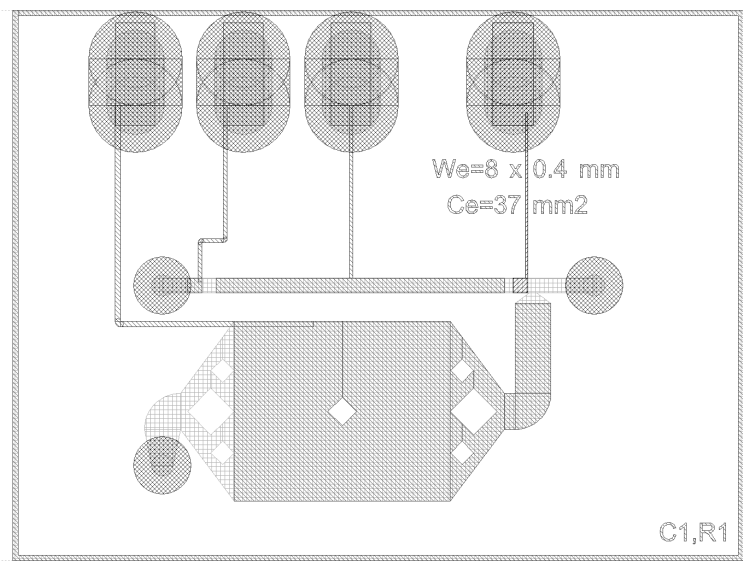

Figure A.2: Chip design 1-1

\section{A.1.2.3 Alignment marks}

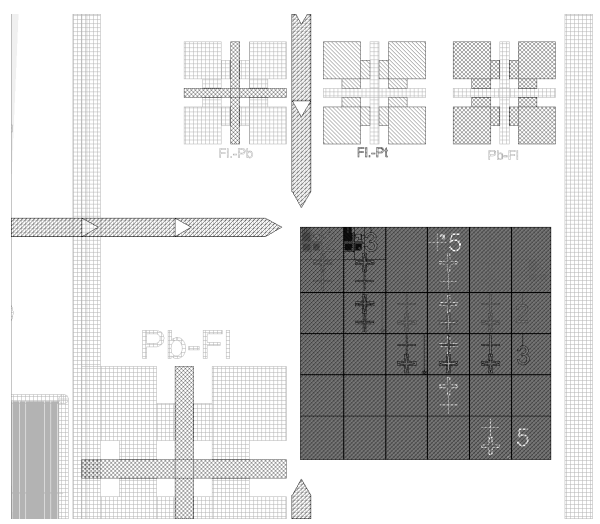

Figure A.3: Zoomed view of the lithographic alignment marks. Special attention is needed to align the IrOx fix layer ( $\mathrm{nr} 5$ ) onto the platinum electrodes ( $\mathrm{nr} 2$ ). 


\section{A.1.2.4 Layers}

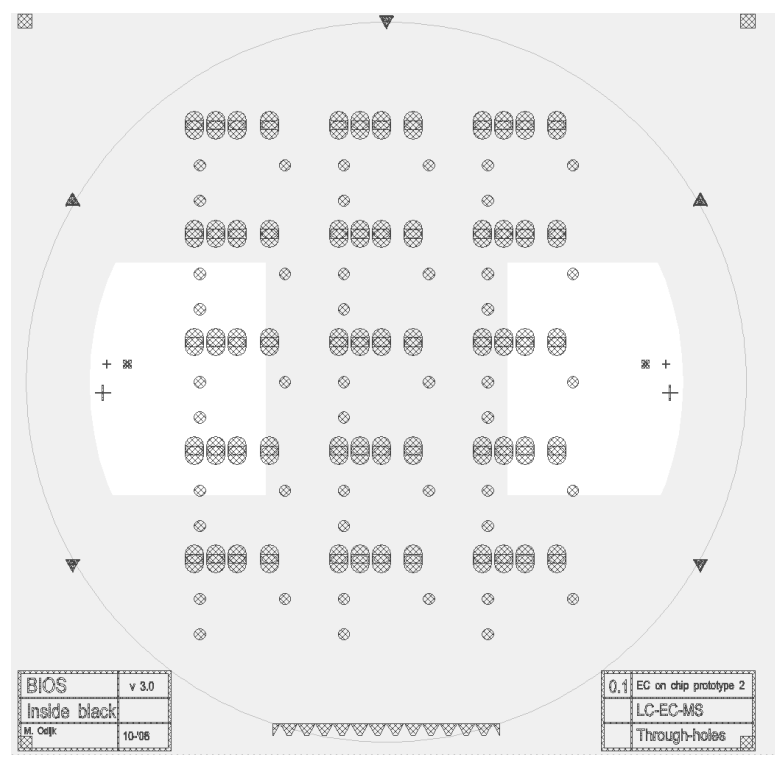

Figure A.4: Mask 0; through holes

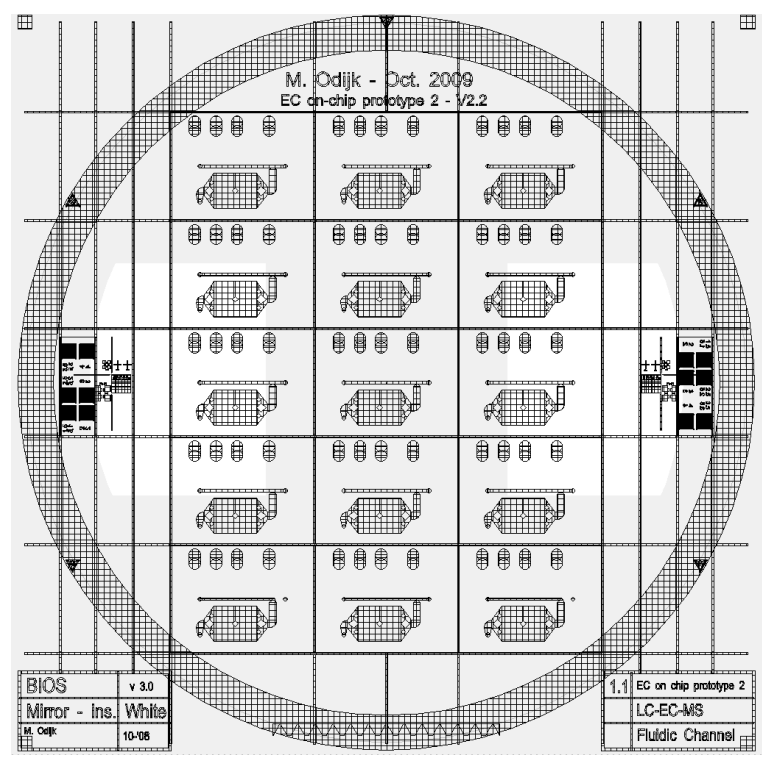

Figure A.5: Mask 1; fluidic channels 


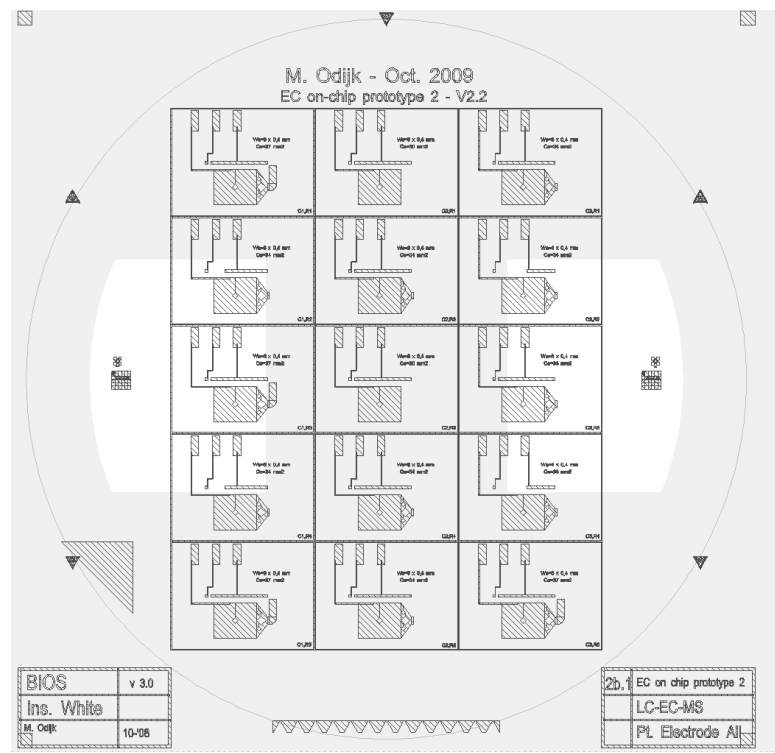

Figure A.6: Mask 2b; All pt electrodes. (Used for platinum work, counter and extra sensing electrode.)

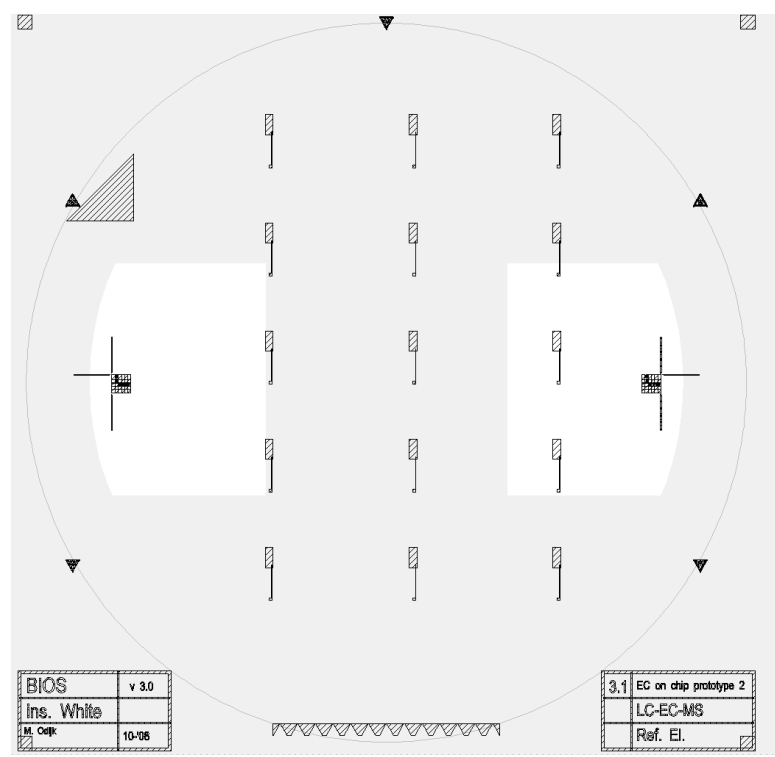

Figure A.7: Mask 3c; Ref. El. (Used for IrOx reference electrode on the right side of the work electrode. This is the preferred setup.) 


\section{A.1.3 Processing steps}

Table A.1: Processing steps Top Wafer.

\begin{tabular}{|c|c|c|c|}
\hline $\mathbf{N r}$ & Process & Parameters & View \\
\hline 1. & $\begin{array}{l}\text { New wafer selec- } \\
\text { tion }\end{array}$ & $\begin{array}{l}\text { This is the standard process for all pt. electrodes. For some } \\
\text { other kind of work electrode material, add an extra sequence of } \\
\text { lithography, sputtering and lift-off with the desired material. } \\
\text { - diameter } 3 \text { inch, borofloat, } 1100 \text { um thickness. These can be } \\
\text { obtained from the general storage in the cleanroom. } \\
\text { - a batch of top wafers consists of } 4 \text { wafers. } \\
\text { - double sided polished } \\
\text { - Keep the small flat to the left and the large flat downwards, to } \\
\text { - Nucess top side. } \\
\text { - Number each wafer on the backside with a diamond pen. }\end{array}$ & \\
\hline 2. & Cleaning & $\begin{array}{l}\text { - Only use the dedicated wafer carriers and glass rod! } \\
\text { - Beaker 1: HNO3 }(100 \%) 5 \mathrm{~min} \\
\text { - Beaker 2: HNO3 }(100 \%) 5 \mathrm{~min} \\
\text { - Quick Dump Rinse }<0.1 \mu \mathrm{S} \\
\text { - Spin drying }\end{array}$ & \\
\hline 3. & $\begin{array}{l}\text { Top side: } \\
\text { Sputtering Cr } \\
(10 \mathrm{~nm}), \text { Sputter- } \\
\text { ing Au (150nm) } \\
\text { (Sputterke) }\end{array}$ & $\begin{array}{l}\text { Cr deposition - Layer thickness } 10 \mathrm{~nm} \\
\text { - Ar flow: } 45 \%=79.3 \mathrm{sccm} \\
\text { - Base pressure: } 9 \mathrm{e}-7 \mathrm{mbar} \\
\text { - } \text { Sputter pressure: } 6.6 \mathrm{e}-3 \mathrm{mbar} \\
\text { - } \text { power: } 200 \mathrm{~W} \\
\text { - } \text { Depositionrate }=10 \mathrm{~nm} / \mathrm{min} \\
\text { - Pre-sputter time }=1 \mathrm{~min} . \\
\text { Au depostion time }=1 \mathrm{~min} . \\
\text { Ar flow: } 45 \%=79.3 \mathrm{sccm} \\
\text { - } \text { Base pressure: } 9 \mathrm{e}-7 \mathrm{mbar} \\
\text { - Sputter pressure: } 6.6 \mathrm{e}-3 \mathrm{mbar} \\
\text { - power: } 200 \mathrm{~W} \\
\text { - Depositionrate }=47 \mathrm{~nm} / \mathrm{min} .(\text { measured by me) } \\
\text { - Pre-sputter time }=1 \mathrm{~min} . \\
\text { Depostion time }=3 \mathrm{~min} .\end{array}$ & \\
\hline
\end{tabular}




\begin{tabular}{|c|c|c|c|}
\hline 4. & $\begin{array}{l}\text { Coating resist, } \\
\text { Lithography } \\
\text { (mask layer } 1 \text { - } \\
\text { mirrored inside } \\
\text { white - labeled } \\
\text { fluidic channels), } \\
\text { Development, } \\
\text { Postbake }\end{array}$ & $\begin{array}{l}\text { - Dehydration bake: O2-plasma 150C, } 15 \mathrm{~min} \text {. } \\
\text { - Spin Resist; type }=\text { olin } 907-17 ; 4000 \mathrm{rpm}, 20 \mathrm{sec} \text {. } \\
\text { - Prebake: hotplate } 95 \mathrm{C} ; 60 \mathrm{sec} \text {. } \\
\text { - On EVG620; choose manual top side, constant time, hard } \\
\text { - Extact, stop after contact. } \\
\text { - After exposure bake: hotplate } 120 \mathrm{C} ; 60 \mathrm{sec} . \\
\text { - Development: OPD4262; } 45-60 \mathrm{sec} . \\
\text { - Spin dry } \\
\text { - Measure the etching tools on the mask with a microscope to } \\
\text { - Pake sure the channels will get the right depth! } \\
\text { - Postbake: } 120 \mathrm{C} ; 30 \mathrm{~min} \text {. }\end{array}$ & E \\
\hline 5. & $\begin{array}{l}\text { Etching } \mathrm{Au} \text {, } \\
\text { Etching } \mathrm{Cr} \text {, } \\
\text { Depth measure- } \\
\text { ment }\end{array}$ & $\begin{array}{l}\text { Au etch: } \\
\text { - UV-Ozone cleaning: } 300 \mathrm{~s} \text { to get more uniform etching. } \\
\text { Right before etching! } \\
\text { - Use standard gold etch from preparation room. } \\
\text { - Take beaker out of the box to see better and prepare a second } \\
\text { beaker with DI to quickly flush with DI to check in between } \\
\text { etching. } \\
\text { - Quick Dump Rinse }<0.1 \mathrm{uS} \\
\text { - Spin drying } \\
\text { - Etchrates between } 300 \text { and } 100 \mathrm{~nm} / \mathrm{min}->\text { time between } 35 \\
\text { and } 1: 30 \text { sec. } \\
\text { - Make sure to remove all gold before etching Cr, but do } \\
\text { not overetch! } \\
\text { Cr etch: } \\
\text { - Use standard Cr etch. Also prepare DI. } \\
\text { - Quick Dump Rinse }<0.1 \mu \mathrm{S} \\
\text { - Spin drying } \\
\text { - Etchrates = } 100 \mathrm{~nm} / \mathrm{min}->5 \mathrm{~s} \text {. } \\
\text { - Measure the etching tools on the mask with a microscope to } \\
\text { make sure the channels will get the right depth. }\end{array}$ & E \\
\hline 6. & $\begin{array}{l}\text { HF etching, 4um } \\
\text { or 3um deep. }\end{array}$ & $\begin{array}{l}\text { - Add dicing foil to backside } \\
\text { - Make } 25 \% \text { HF solution }->1 \mathrm{~L} \mathrm{DI+50 \%} \mathrm{HF} \mathrm{solution,} \mathrm{water} \\
\text { first!! } \\
\text { - Etch rate }->1.25 \text { um/min. (JAN), but decreasing with approx. } \\
20 \% \text { in speed after each wafer. } \\
\text { - Check transparency of etching tools to control depth } \\
\text { - Remove dicing foil to prevent contamination of the dektak, } \\
\text { measure depth, write values down }\end{array}$ & \\
\hline
\end{tabular}




\begin{tabular}{|c|c|c|c|}
\hline $\mathrm{Nr}$ & Process & Parameters & View \\
\hline 7. & $\begin{array}{l}\text { Laminating with } \\
\text { powderblast } \\
\text { foil, lithog- } \\
\text { raphy (mask } \\
\text { layer 5: Top - } \\
\text { contact holes), } \\
\text { Development }\end{array}$ & $\begin{array}{l}\text { - Select foil BF410 (see fridge) } \\
\text { - On the laminator select; Temp } 130 \mathrm{C} \text { ('Carry' preset), Speed: } \\
2 \text { ('Carry' preset) } \\
\text { - EVG620 mask aligner: Use bottomside, crosshair, softcon- } \\
\text { tact, } 40 \text { um separation, } 1 \mathrm{~mm} \text { thickness, continuous. } \\
\text { - Exposure time: } 20 \mathrm{sec} \\
\text { - After exposure, remove protection foil by heating the wafer } \\
\text { to } 90 \mathrm{C} \text { for approx. } 20 \mathrm{sec} \text {. } \\
\text { - Add dicing foil to backside of wafer. } \\
\text { ELTN } 7143-4 \text { HCM Spray Developer } \\
\text { - Replace spray solution if necessary. Na2CO3 : H2O = 15g : } \\
\text { - } 7.5 \text { liters (+ } 1 \text { cup Antifoam) } \\
\text { - Temp: } 32 \mathrm{C} \\
\text { - Dime: } 2 \text { to } 3 \text { min } \\
\text { - ter half the time } \\
\text { - Rinse and spin dry }\end{array}$ & D \\
\hline 8. & $\begin{array}{l}\text { Powder Blasting } \\
\text { through holes }\end{array}$ & $\begin{array}{l}\text { ELTN10156 / Powderblaster } \\
\text { - Particles: } 29 \mathrm{um} \\
\text { - Pressure: } 4.6 \mathrm{bar} \\
\text { - Massflow: } 3-12 \mathrm{~g} / \mathrm{min} \\
\text { - Etchrate appr. } 29 \mathrm{um} \text { per } \mathrm{g} / \mathrm{cm} 2 \\
\text { - Approximately } 550 \text { gr. powder for } 1 \text { wafer of } 1100 \mathrm{um} \text { thick- } \\
\text { ness. }\end{array}$ & \\
\hline 9. & $\begin{array}{l}\text { Foil, Resist, } \mathrm{Au} \text {, } \\
\text { Cr stripping }\end{array}$ & $\begin{array}{l}\text { - Remove P.B. foil and dicing foil, if possible by hand but be } \\
\text { careful! } \\
\text { - Clean in aceton or DI in ultrasonic for } 1 \text { hour to remove par- } \\
\text { ticles and prevent contamination of the HNO3. } \\
\text { - Strip resist in } 100 \% \text { HNO3 } \\
\text { - QDRinse } \\
\text { - Strip Au in Au-ets } \\
\text { - QDRinse } \\
\text { - Strip Cr. in Cr.-ets } \\
\text { - QDRinse } \\
\text { - Clean wafers in ultrasonic vlsi IPA for } 60 \mathrm{~min} \text {. } \\
\text { - QDRinse } \\
\text { - Spin Dry }\end{array}$ & \\
\hline
\end{tabular}


Table A.2: Processing steps bottom Wafer.

\begin{tabular}{|c|c|c|c|}
\hline $\mathrm{Nr}$ & Process & Parameters & View \\
\hline 1. & $\begin{array}{l}\text { New wafer selec- } \\
\text { tion }\end{array}$ & $\begin{array}{l}\text { - diameter } 3 \text { inch, borofloat } 1100 \text { um thickness. These can be } \\
\text { obtained from Jan van } \mathrm{N} \text {. } \\
\text { - a batch of top wafers consists of } 6 \text { wafers. } \\
\text { - double sided polished } \\
\text { - Keep small flat to the left and large flat downwards, to process } \\
\text { the topside. } \\
\text { - Number each wafer on the backside with a diamond pen. }\end{array}$ & \\
\hline 2. & Cleaning & $\begin{array}{l}\text { - Only use the dedicated wafer carriers and glass rod! } \\
\text { - Beaker 1: HNO3 }(100 \%) 5 \mathrm{~min} \\
\text { - Beaker 2: HNO3 }(100 \%) 5 \mathrm{~min} \\
\text { - Quick Dump Rinse }<0.1 \mu \mathrm{S} \\
\text { - Spin drying }\end{array}$ & \\
\hline 3. & $\begin{array}{l}\text { Coating resist, } \\
\text { Lithography } \\
\text { (mask layer } 2 b \\
\text { - labeled all pt), } \\
\text { Development, } \\
\text { Postbake }\end{array}$ & $\begin{array}{l}\text { - Dehydration bake: O2-plasma 150C, } 15 \mathrm{~min} \text {. } \\
\text { - Spin Resist; type = olin 907-17; 4000rpm, } 20 \mathrm{sec} \text {. } \\
\text { - Prebake: hotplate } 95 \mathrm{C} ; 60 \mathrm{sec} \text {. } \\
\text { - On EVG620; choose manual top side, constant time, hard } \\
\text { contact, stop after contact. } \\
\text { - Exposure parameters: } 4.5 \mathrm{sec} . \\
\text { - After exposure bake: hotplate } 120 \mathrm{C} ; 60 \mathrm{sec} . \\
\text { - Development: OPD4262; } 45-60 \mathrm{sec} . \\
\text { - Spin dry } \\
\text { - Postbake: } 120 \mathrm{C} ; 30 \mathrm{~min} .\end{array}$ & \\
\hline 4. & $\begin{array}{l}\text { BHF-etching } \\
\text { (140nm deep) }\end{array}$ & $\begin{array}{l}\text { - Ozone cleaning } 300 \text { s. } \\
\text { - Add dicing foil to backside of wafer. } \\
\text { - } \text { stch time is } 6 \text { min. in stirred solution without heating. Renew } \\
\text { - Remove foil afterwards. }\end{array}$ & \\
\hline 5. & $\begin{array}{l}\text { Sputtering Ta } \\
(10 \mathrm{~nm}), \text { Sputter- } \\
\text { ing Pt (120nm) } \\
\text { (Sputterke) }\end{array}$ & $\begin{array}{l}\text { - Cover dummy wafer partly with a broken piece of wafer } \\
\text { waste. } \\
\text { - First sputter dummy wafer. } \\
\text { - Do lift off process for dummy wafer, measure electrode } \\
\text { height, hole depth. } \\
\text { - Adjust sputter times } \\
\text { Sputtering Ta } \\
\text { - Ar flow: } 45 \%=80 \mathrm{sccm} \\
\text { - Base pressure: } 9 \mathrm{E}-7 \mathrm{mbar} \\
\text { - Sputter pressure: } 6.6 \mathrm{e}-3 \mathrm{mbar} \\
\text { - power: } 200 \mathrm{~W}, \text { Compensate deposition time if power not } \\
\text { - } \text { reached. } \\
\text { - Depositionrate }=16.5 \mathrm{~nm} / \mathrm{min} \\
\text { - Deposition time }=37 \mathrm{sec} \text {. }\end{array}$ & \\
\hline
\end{tabular}


Table A.3: Processing steps bottom Wafer - part 2.

\begin{tabular}{|c|c|c|c|}
\hline $\mathbf{N r}$ & Process & Parameters & View \\
\hline 5. & $\begin{array}{l}\text { Sputtering Ta } \\
(10 \mathrm{~nm}) \text {, Sputter- } \\
\text { ing Pt (120nm) } \\
\text { (Sputterke) }\end{array}$ & $\begin{array}{l}\text { Sputtering } P t \\
\text { - Ar flow: } 45 \%=80 \mathrm{sccm} \\
\text { - Base pressure: } 9 \mathrm{E}-7 \mathrm{mbar} \\
\text { - Sputter pressure: } 6.6 \mathrm{e}-3 \mathrm{mbar} \\
\text { - power: } 200 \mathrm{~W} \\
\text { - Depositionrate }=24.9 \mathrm{~nm} / \mathrm{min} \\
\text { - pre-sputter time }=1 \mathrm{~min} . \\
\text { - Deposition time }=4: 49 \mathrm{~min} \text {. (Still proven to be a good value } \\
\text { in aug. } 2009, \text { combined with the BHF etching.) }\end{array}$ & \\
\hline 6. & Lift-off & $\begin{array}{l}\text { - Prepare } 2 \text { beakers with aceton } \\
\text { - Strip resist in ultrasonic bath } \\
\text { - Flush with demi-water } \\
\text { - Strip remaining resist in beaker } 2 \text { in ultrasonic bath } \\
\text { - Flush with demi-water } \\
\text { - Quick dump rinse. }<0.1 \mathrm{uS} \\
\text { - Clean in } 100 \% \text { HNO3 } \\
\text { - Quick dump rinse. }<0.1 \mathrm{uS}\end{array}$ & \\
\hline 7. & $\begin{array}{l}\text { Coating resist, } \\
\text { Lithography } \\
\text { (mask layer } 3 c- \\
\text { labeled Ref. El.), } \\
\text { Development, } \\
\text { Postbake }\end{array}$ & $\begin{array}{l}\text { - Dehydration bake: O2-plasma 150C, } 15 \mathrm{~min} . \\
\text { - Spin Resist; type = olin 907-17; 4000rpm, 20sec. } \\
\text { - Prebake: hotplate } 95 \mathrm{C} ; 60 \mathrm{sec} . \\
\text { - On EVG620; choose manual top side, constant time, hard } \\
\text { contact, stop after contact. } \\
\text { - Exposure parameters: } 4.5 \mathrm{sec} . \\
\text { - After exposure bake: hotplate } 120 \mathrm{C} ; 60 \mathrm{sec} . \\
\text { - Development: OPD4262; } 45-60 \mathrm{sec} \text {. } \\
\text { - Spin dry, Postbake: } 120 \mathrm{C} ; 30 \mathrm{~min} .\end{array}$ & \\
\hline 8. & $\begin{array}{l}\text { BHF-etching } \\
\text { (214nm deep) }\end{array}$ & $\begin{array}{l}\text { - Ozone cleaning } 300 \mathrm{~s} \text {. } \\
\text { - Add dicing foil to backside of wafer. } \\
\text { Etch time is 8:40 min. in stirred solution without heating. } \\
\text { Renew solution after } 4 \text { wafers. } \\
\text { - Remove foil afterwards. }\end{array}$ & \\
\hline 9. & 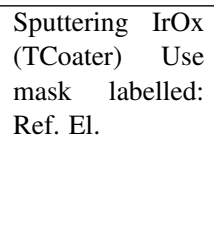 & $\begin{array}{l}\text { See A. } 2 \text { for details. } \\
\text { - Do an ozone cleaning for } 300 \text { s to remove some organic } \\
\text { residues. Especially important if the wafers have been in } \\
\text { the box for a while! } \\
\text { - Use settings from run } 6 \text {, with timings } 4 / 12 / 4 / 30 \mathrm{~min} \text {. } \\
\text { - Ar settings } 80 / 80 / 80 / 10+10,020 / 0 / 0 / 20 \text {. } \\
\text { - Power: } 200 / 200 / 200 / 50 \mathrm{~W} \text {. }\end{array}$ & \\
\hline
\end{tabular}


Table A.4: Processing steps bottom Wafer - part 2.

\begin{tabular}{|c|c|c|c|}
\hline $\mathrm{Nr}$ & Process & Parameters & View \\
\hline 10 . & $\begin{array}{l}\text { Lift-off } \\
\text { nat }\end{array}$ & 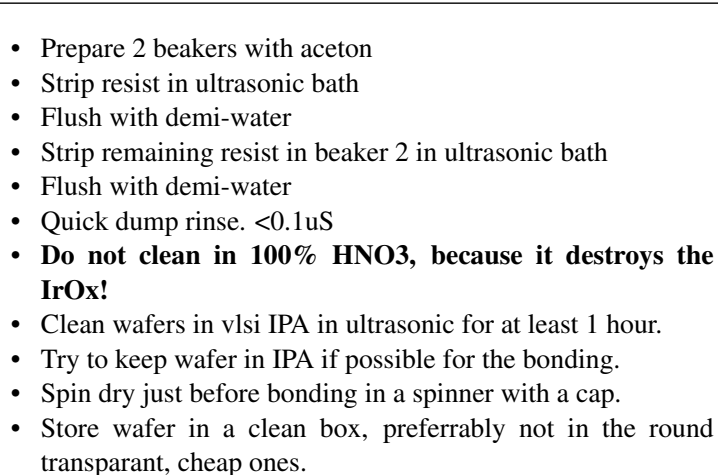 & \\
\hline
\end{tabular}


Table A.5: Bonding.

\begin{tabular}{|c|c|c|c|}
\hline $\mathrm{Nr}$ & Process & Parameters & View \\
\hline 1. & $\begin{array}{l}\text { Special } \\
\text { Cleaning }\end{array}$ & $\begin{array}{l}\text { Cleaning P.B. wafers } \\
\text { Do a short KOH dip of } 20 \mathrm{sec} \text {. in the cleanest } \mathrm{KOH} \text { beaker near the } \\
\text { automated RCA cleaning. } \\
\text { - QDR } \\
\text { - } 100 \% \text { HNO3 @ pyrex cleaning } 5 \text { min. beaker } 1,5 \mathrm{~min} \text {. beaker } 2 . \\
\text { - QDR } \\
\text { - Spin dry in spinner with built-in N2 gun. Dry only shortly before } \\
\text { the bonding. Keep in DI in between. }\end{array}$ & \\
\hline 2. & Aligning & $\begin{array}{l}\text { - Choose special anodic bond program on EVG620. Choose anodic } \\
\text { bond, flag before WEC, crosshair, align before WEC. } \\
\text { - Use the special bond tool (holes for vacuum to the back of the } \\
\text { machine), the special bond chuck and glass bond tool. Also use } \\
\text { the special ruler for bonding. } \\
\text { - Pay attention to the alignment and straightness of the spacers and } \\
\text { clamps on the bond chuck. } \\
\text { - The wafer with the powderblasted holes goes first. Fluidic channels } \\
\text { (topside) facing downwards. } \\
\text { - Follow instructions on-screen. } \\
\text { - Insert bottom wafer, but shift it approx. } 1 \mathrm{~mm} \text { to the right to com- } \\
\text { pensate for the small flat. } \\
\text { - Make sure not to bend the spacers when you have to move them in. } \\
\text { When removing the chuck, make sure to do this carefully. Rotate } \\
\text { the chuck but make sure not to touch the two clamps. }\end{array}$ & \\
\hline 3. & Prebond & $\begin{array}{l}\text { - Make a prebond by pressing onto the wafer with a tweaser. } \\
\text { - Once a prebond is formed, carefully remove the flags and move the } \\
\text { wafer stack to a black underground. Make sure that the powderblast } \\
\text { wafer is at the bottom. } \\
\text { - Improve the prebond by pressing onto the wafer with a tweaser. } \\
\text { - Optionally the prebond can be improved by putting the wafer stack } \\
\text { inside the hot press @ } 11 \text { ton near sputterke. } \\
\text { - Make a direct bond by annealing the wafer stack @ 600C for } 1 \\
\text { hour at the oven at EL/TN floor } 10 \text {. Ramp up time 6hours, ramp } \\
\text { down time } 12 \text { hours. }\end{array}$ & \\
\hline 4. & Dicing & $\begin{array}{l}\text { Mount the wafer stack onto a holder using the thick, transparant } \\
\text { dicing foil from Jan, to prevent sharp edges on the side of the chips. } \\
\text { Powderblast holes go onto the foil to seal them off. } \\
\text { - Use disco dicing saw. } \\
\text { - Do a blade maintenance. The second entry is the carbon blade; } \\
\text { type TC300. } \\
\text { - Select device data list. Rotational speed } 25000 \mathrm{rpm}, 3 \mathrm{~m} / \mathrm{s} \text { feed, } \\
\text { x-translation=20.3 mm, y-translation=15.3 mm, substrate thick- } \\
\text { ness } 1650 u m, \text { foil thickness } 80 u m \text { (lowest allowed to prevent sharp } \\
\text { edges on chip), } 55 \mathrm{um} \text { spacing. } \\
\text { - Cut wafers. The total stack is } 2.2 \mathrm{~mm} \text { thick. It is not recommended } \\
\text { to do this in one cut (you brake a lot of blades). A better option is } \\
\text { to dice down to half the stack thickness, fix the partly diced stack } \\
\text { again to a new piece of foil and than dice the other half. }\end{array}$ & \\
\hline
\end{tabular}




\section{A.2 IrOx sputtering and adhesion study}


Table A.6: IrOx sputter parameters and results using TCoater sputtering machine. Values listed for power, Ar/O2, thickness, rate and valve are specified for the IrOx sputtering alone, except for runs 2 and 3.

\begin{tabular}{|c|c|c|c|c|c|c|c|c|c|c|c|c|c|c|}
\hline Run & time $^{a}$ & layers & guns & power $(\mathrm{W})$ & Base $\mathrm{P}^{b}$ & sputter p. & $\mathrm{Ar} / \mathrm{O} 2^{c}$ & valve & Dist $^{d}$ & $\operatorname{Rot}^{e}$ & Temp. & color & thickn. & rate $^{f}$ \\
\hline 1 & $2+20$ & $\mathrm{Ti} / \mathrm{Ir}$ & $2+3$ & $40 \& 96$ & 1E-6 & $6.6 \mathrm{E}-3$ & $80+20 / 20$ & $45 \%$ & 44 & 5 & $20^{\circ} \mathrm{C}$ & brown & ? & ? \\
\hline 2 & 25 & $\mathrm{Ti}$ & 2 & 200 & $1 \mathrm{E}-6$ & $6.6 \mathrm{E}-3$ & $80 / 0$ & $65 \%$ & 44 & 5 & $20^{\circ} \mathrm{C}$ & grey & 58 & 2.3 \\
\hline 3 & $2+15$ & $\mathrm{Ti} / \mathrm{Pt}$ & $2+1$ & $200 / 200$ & $1 \mathrm{E}-6$ & $6.6 \mathrm{E}-3$ & $80 / 0$ & $65 \%$ & 44 & 5 & $20^{\circ} \mathrm{C}$ & silver & $5+133$ & 8.9 \\
\hline 4 & $4 / 12 / 4 / 45$ & $\mathrm{Ti} / \mathrm{Pt} / \mathrm{Ti} / \mathrm{Ir}$ & $2+1+3$ & 100 & $1 \mathrm{E}-6$ & $6.6 \mathrm{E}-3$ & $20 / 20$ & $45 \%$ & 44 & 5 & $20^{\circ} \mathrm{C}$ & grey/brown & 238 & 5.3 \\
\hline 5 & $4 / 12 / 4 / 20$ & $\mathrm{Ti} / \mathrm{Pt} / \mathrm{Ti} / \mathrm{Ir}$ & $2+1+3$ & 100 & $1 \mathrm{E}-6$ & $3.3 \mathrm{E}-3$ & $10+10 / 20$ & $64 \%$ & 44 & 5 & $20^{\circ} \mathrm{C}$ & grey/brown & 100 & 5.0 \\
\hline 6 & $4 / 12 / 4 / 30$ & $\mathrm{Ti} / \mathrm{Pt} / \mathrm{Ti} / \mathrm{Ir}$ & $2+1+3$ & 50 & $1 \mathrm{E}-6$ & $3.3 \mathrm{E}-3$ & $10+10 / 20$ & $68 \%$ & 44 & 5 & $20^{\circ} \mathrm{C}$ & grey/blue & 73 & 2.43 \\
\hline 7 & $4 / 15$ & $\mathrm{Ti} / \mathrm{Ir}$ & $2+3$ & 50 & $1 \mathrm{E}-6$ & $3.3 \mathrm{E}-3$ & $30+10 / 0$ & $\sim 68 \%$ & 44 & 5 & $20^{\circ} \mathrm{C}$ & silver & 17 & 1.13 \\
\hline 8 & $4 / 15$ & $\mathrm{Ti} / \mathrm{Ir}$ & $2+3$ & 50 & $1 \mathrm{E}-6$ & $3.3 \mathrm{E}-3$ & $10+10 / 10$ & $43 \%$ & 44 & 5 & $20^{\circ} \mathrm{C}$ & darksilver & 37.5 & 2.5 \\
\hline 9 & $4 / 12 / 4 / 30$ & $\mathrm{Ti} / \mathrm{Pt} / \mathrm{Ti} / \mathrm{Ir}$ & $2+1+3$ & 50 & $1 \mathrm{E}-6$ & $3.3 \mathrm{E}-3$ & $5+5 / 20$ & $?$ & 44 & 5 & $20^{\circ} \mathrm{C}$ & black/grey & 77 & 2.56 \\
\hline
\end{tabular}

\footnotetext{
${ }^{a}$ Sputter time in minutes

${ }^{b}$ Pressures in mBar

${ }^{c}$ Gases (in sccm) can be introduced either directly into chamber or from the sputter gun.

${ }^{d}$ distance from base point of substrate in $\mathrm{mm}$

${ }^{e}$ rotation of substrate in rpm

${ }^{f}$ Sputter rate of (if not clear $\mathrm{IrOx}$ ) in $\mathrm{nm} / \mathrm{min}$
} 


\section{A.3 List of standard amino acids}

\section{standard Amino Acids}
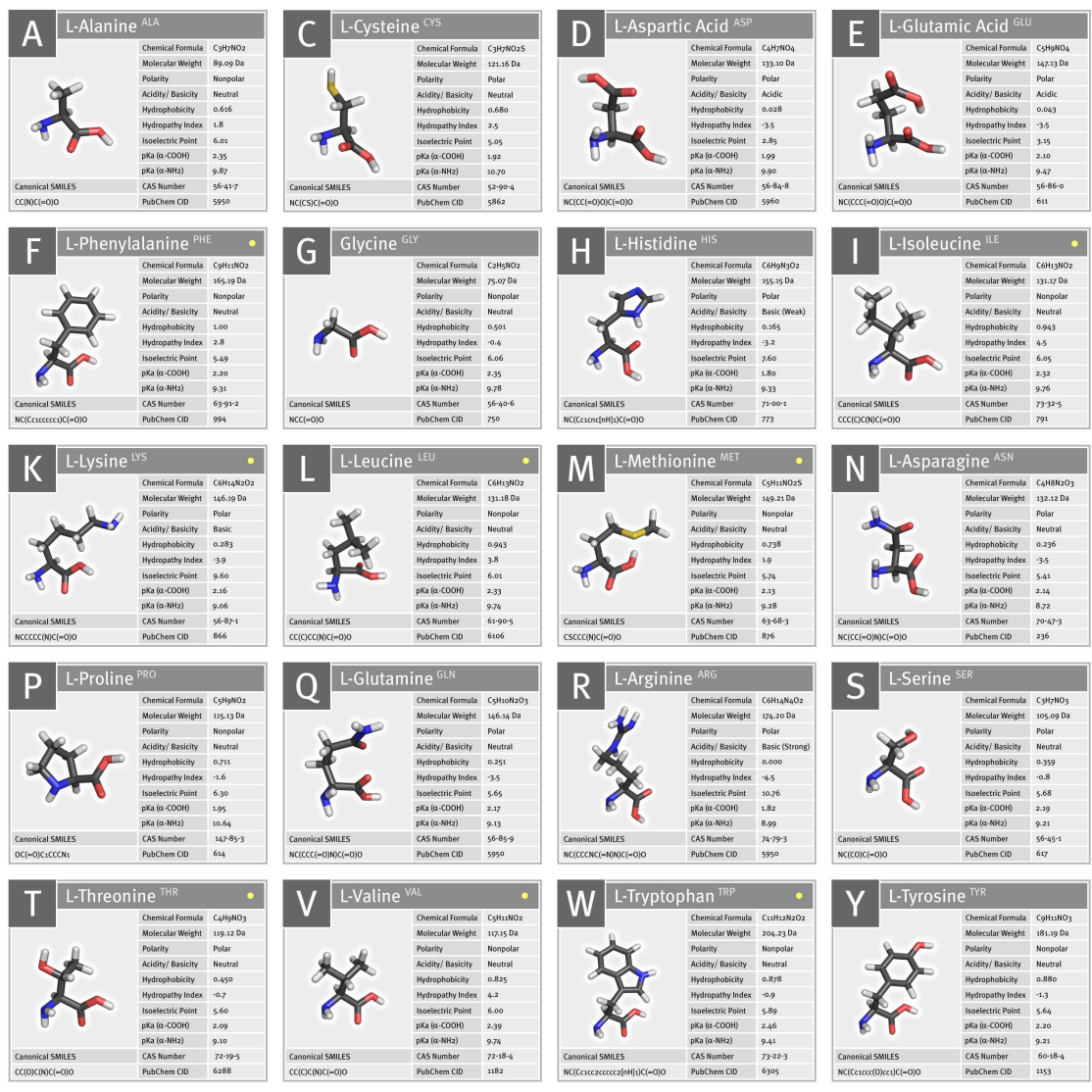

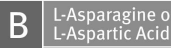




\section{Abstract}

This thesis describes the design of a small flow-through electrochemical cell in a lab-on-chip format. This cell is aimed at two applications.

The first application lies in drug screening. 75\% of the marketed drugs are metabolized by an oxidative mechanism in the liver by enzymes of the Cytochrome P450 (CYP450) family. In the pre-clinical phase of new drug development, identification of the metabolic products is important. One of the ways to mimic the metabolic mechanism of CYP450 in vitro is to use direct electrochemical oxidation using an electrochemical cell. Although direct oxidation can not be compared directly to enzymatic oxidation, it can be used to screen drugs at an early stage of development.

The second application lies in the field of proteomics. The most frequently used method in proteomics to study complex mixtures of proteins, is to use enzymatic digestion to cleave the proteins into smaller parts which can be subsequently detected by mass spectrometry. This method relies on a predictable cleavage process, e.g. provided by the enzyme trypsin. Electrochemical cleavage using an electrochemical cell is an alternative, complementary method, providing the advantage of a fully instrumental approach.

In chapter three, a novel miniaturized electrochemical cell design is presented, fabricated out of two glass substrates. This cell contains a complete, integrated 3 -electrode setup with platinum working and counter electrodes and a palladium pseudo-reference electrode. The cell has sub-microliter internal volumes and is aimed for high turnover rates of the introduced chemical species.

Using this cell, the turnover rate of small and fast reacting ions is studied and found to be near to $100 \%$ using UV-vis spectroscopy as detection method. Also, the cell is used for drug metabolism studies. The direct oxidation of amodiaquine, a known antimalarial agent, is investigated and compared to results obtained using commercially available cells. The chip showed similar performance, while using less analyte.

In chapter four, an improved cell design is presented using iridium oxide (IrOx) as pseudo-reference material. The performance of this device is characterized using potentiometric measurements. The IrOx electrode shows a long-term potential 
variation of $5 \mathrm{mV}$ per 20 minutes, corresponding to the time required for a typical conversion experiment. Using this chip, a second compound, procainamide, a known antiarrythmic drug, is tested to show the feasibility for drug screening. The results obtained with the improved chip are compared to results obtained with rat and human liver cell microsomal incubations. Only two out of four known metabolites are observed from microsomal incubation, while direct oxidation generates all four metabolites. These results indicate that direct electrochemical oxidation using miniaturized electrochemical cells can be used as complementary in-vitro method in early stages of drug development. The direct method is faster and cheaper and allows for on-line analysis in an automated fashion.

Also presented in chapter four is a portable measurement setup. This setup is developed to precisely control fluid flows over the chip at continuous rates down to a few nanoliter per minute. This setup is referred to as the Lab-in-a-Suitcase, and contains two syringe pumps, two switching valves and one injection valve, a potentiostat and a holder to interface the chip. The total dead volume of all connecting capillaries and microfluidic connectors is reduced to a volume below $5 \mu \mathrm{L}$. The setup is controlled by a laptop running a single Labview program that allows full automation of measurements and cleaning protocols.

Chapter five describes preliminary work of tyrosine specific electrochemical cleavage of proteins as alternative to tryptic digestion using the chip. Some cleavage of the tripeptide Leucine-Tyrosine-Leucine is observed, although future work requires a redesign of the chip and the surrounding equipment for this specific task to get sufficient cleavage yields.

In chapter six, redox cycling is investigated as a tool to measure electrochemical compounds with a high selectivity and high sensitivity. Using this phenomena, a new measurement method named differential cyclic voltammetry (DCV) is proposed. This method provides easy and simultaneous detection of concentrations of redox cyling compounds with a high selectivity. The method is investigated both theoretically using finite element computer simulations and experimentally using an interdigitated array electrode.

Chapter seven proposes future plans to develop an integrated chip using polymer substrates containing an electrochemical cell, an electrospray ionization needle and possibly a separation column. This future chip can contain different working electrode materials like e.g. doped diamond. 


\section{Samenvatting}

Dit proefschrift is gewijd aan het ontwerp van een kleine elektrochemische cel met continue vloeistofstroom in een Lab-on-Chip formaat. Deze cel is ontworpen voor twee verschillende toepassingen.

De eerste toepassing is het testen en opsporen van nieuwe medicijnen. $75 \%$ van alle op dit moment op de markt verkrijgbare medicijnen worden omgezet door een oxidatief mechanisme in de lever, bewerkstelligd door enzymen van de Cytochroom P450 familie. In de pre-klinische fase van het ontwikkelen van nieuwe medicijnen is het identificeren van omzettingsproducten (metabolieten) belangrijk. Eén van de manieren om dit oxidatieve mechanisme in vitro na te bootsen is door directe elektrochemische omzetting in een elektrochemische cel. Alhoewel deze directe methode niet volledig vergelijkbaar is met de enzymatische omzetting, kan het toch gebruikt worden om nieuwe medicijnen te testen.

De tweede toepassing heeft te maken met het onderzoeksveld van proteomics. De meest gebruikte onderzoeksmethode in proteomics voor het bestuderen van complexe mengsels van eiwitten is het gebruik van enzymatische opsplitsing. Bij deze methode worden eiwitten in kleinere stukken geknipt waarna ze kunnen worden gedetecteerd in een massaspectrometer. Het succes van deze methode hangt af van een betrouwbare, voorspelbare splitsing, die bijvoorbeeld kan worden gerealiseerd met behulp van het enzym trypsine. Het is ook mogelijk om eiwitten in kleinere stukken te knippen met behulp van een elektrochemische reactie. Deze alternatieve methode heeft als voordeel dat zij volledig instrumenteel en automatiseerbaar is.

In hoofdstuk drie wordt een nieuw ontwerp van een geminiaturiseerde elektrochemische cel gepresenteerd. Deze cel is vervaardigd uit twee glazen substraten. De cel bevat een complete 3-elektrode configuratie met platina werk- en counterelektrodes en een palladium pseudoreferentie-elektrode. De cel heeft een intern volume van minder dan 1 microliter en is met name gericht op hoge omzettingsefficiënties van de inkomende elektrochemisch actieve stoffen.

Met behulp van deze cel is de omzettingsefficiëntie bestudeerd voor kleine en snel reagerende ionen. Voor deze stoffen is de omzetting dicht bij 100\%, waarbij UV-vis 
spectroscopie is gebruikt als detectie methode. Directe oxidatie van amodiaquine, een bekend medicijn tegen malaria, is onderzocht en vergeleken met resultaten van commercieel verkrijgbare cellen. De chip vertoonde een vergelijkbaar resultaat met minder verbruik van de om te zetten stof.

In hoofdstuk vier wordt een verbeterde versie van dit celontwerp gepresenteerd, waarbij palladium is vervangen door iridiumoxide ( $\mathrm{IrOx}$ ) als pseudo-referentie elektrode materiaal. Het gedrag van IrOx is gekenschetst door middel van potentiometrische metingen. Deze elektrode toonde een lange-termijn variatie van $5 \mathrm{mV}$ per 20 minuten, wat overeenkomt met de tijd van een typisch conversie-experiment. Een tweede medicijn dat gebruikt wordt bij hartritmestoornissen, genaamd procainamide, is getest met behulp van deze chip. De verkregen resultaten zijn vergeleken met incubaties van het medicijn met rat en menselijke levercel extracten. Beide incubaties laten slechts 2 van de 4 bekende reactieproducten zien, terwijl directe elektrochemische oxidatie alle 4 reactieproducten geeft. Dit resultaat is daarom een indicatie dat directe elektrochemische oxidatie gebruikt kan worden als aanvullende in vitro methode in de vroege stadia van medicijnontwikkeling. De directe methode is sneller en goedkoper en kan op een geautomatiseerde manier worden uitgevoerd.

Tevens gepresenteerd in hoofdstuk vier is een gemakkelijk te verplaatsen meetopstelling. Deze opstelling is speciaal ontwikkeld om zeer nauwkeurige vloeistofstromen door de chip te bewerkstelligen tot op enkele nanoliters per minuut nauwkeurig. De meetopstelling heeft de naam Lab-in-a-Suitcase (lab in een koffer) gekregen. Deze bevat twee spuitpompen, twee schakelende kleppen, een injectieklep, een potentiostaat en een chiphouder. Het totale dode volume van alle verbindende capillairen en vloeistofaansluitingen is teruggebracht tot minder dan 5 microliter. De opstelling wordt bestuurd door een enkel Labview programma dat volledige automatisering van metingen en schoonmaakprocedures mogelijk maakt.

In hoofdstuk vijf worden voorlopige onderzoeksresultaten gepresenteerd waarbij tyrosine-specifieke eiwitsplitsing met gebruik van elektrochemische oxidatie is onderzocht. Voor deze studie is het tripeptide Leucine-Tyrosine-Leucine gebruikt, waarbij beperkte hoeveelheden splitsingsproduct zijn waargenomen. Toekomstig werk vereist dat de chip wordt herontworpen voor dit specifieke doel om de splitsingsefficiëntie te verhogen. Daarnaast moet de koppeling tussen de chip en de massaspectrometer worden verbeterd om een beter resultaat te verkrijgen.

In hoofdstuk zes wordt het 'redox cycling' effect bestudeerd als middel om elektrochemisch actieve stoffen te detecteren met een hoge detectielimiet en selectiviteit. Met behulp van dit effect wordt een nieuwe meetmethode geïntroduceerd: differentiële cyclische voltammetrie (DCV). Deze meetmethode maakt het mogelijk om op 
eenvoudige wijze verscheidene concentraties van verschillende stoffen gelijktijdig vast te stellen met een hoge selectiviteit. Deze methode is zowel theoretisch, met behulp van computersimulaties, als experimenteel onderzocht met gebruik van een 'interdigitated array' elektrode.

In hoofdstuk zeven worden toekomstige plannen voorgesteld voor een geïntegreerde chip, vervaardigd uit een plastic substraat waar een elektrochemische cel, een elektrospray ionisatie naald en mogelijk een chromatografie kolom op wordt gecombineerd. Deze toekomstige chip kan verschillende werkelektrode materialen bevatten zoals bijvoorbeeld gedoteerd diamant. 



\section{List of publications}

\section{Papers}

M. Odijk, W. Olthuis, V. A. T. Dam and A. van den Berg, Simulation of RedoxCycling Phenomena at Interdigitated Array (IDA) Electrodes:Amplification and Selectivity, Electroanalysis, 20 (5) 2007, 463-468, (Chapter 6).

M. Odijk, A. Baumann, W. Lohmann, F. T. G. van den Brink, W. Olthuis, U. Karst and A. van den Berg, A microfluidic chip for electrochemical conversions in drug metabolism studies, Lab on Chip, 9 2009, 1687-1693, (chapter 3).

M. Odijk, A. Baumann, W. Olthuis, A. van den Berg and U. Karst, Electrochemistryon-chip for on-line conversions in drug metabolism studies, Biosensors and Bioelectronics, 26 2010, 1521-1527, (chapter 4).

\section{Conference contributions}

M. Odijk, J. Everse, W. Olthuis and A. van den Berg, A Simple, Low-Cost Method for High Redox Cycling Amplification Measurements in Hydrogels, In: 22nd International Conference Eurosensors, XXII 2008 Dresden, ISBN 978-3-00-025217-4, (chapter 6).

M. Odijk, W. Olthuis, C.E. van Eijkeren and A. van den Berg, Electrochemistryon-chip for on-line, high-turnover conversions in drug metabolism studies, In: 20th world congress on Biosensors, 2010 Glasgow, (chapter 4).

M. Odijk, H. de Boer, W. Olthuis and A. van den Berg, Lab-in-a-Suitcase for Drug Screening and Proteomics Applications, In: 14th International Conference on Miniaturized Systems for Chemistry and Life Sciences, 2010 Groningen, ISBN 978-09798064-3-8, (chapter 4 \& 5). 
M. Odijk, J. Wiedemair, M.J.J. van Megen, W. Olthuis and A. van den Berg, Differential Cyclic Voltammetry - a Novel Technique for Selective and Simultaneous Detection using Redox Cyling Based Sensors, In: IEEE sensors conference, 2010 Hawaii, ISBN 978-1-4244-8168-2/10, (chapter 6).

C. Salm, J. Eijkel, F. van der Heijden, E. Faber, M. Odijk, Adapting to a Changing Highschool Population, In: 8th European Workshop on Microelectronics Education, 2010. 


\section{Dankwoord}

Dit dankwoord wordt waarschijnlijk toch het meest gelezen deel, maar de schrijfstijl in de meeste proefschriften is daarentegen vaak weinig origineel.

Vandaar dat dit dankwoord op rijm is gezet, zodat er tenminste goed op wordt gelet.

$\mathrm{Nu}$ de vier jaar van mijn AIO periode bijna zijn verstreken, wordt het tijd om mijn waardering naar allerlei mensen uit te spreken.

Wouter, in de afgelopen vijf jaar, stond je altijd voor me klaar.

Vele rode pennen heb je op mijn teksten versleten, maar jouw correcte, opbouwende feedback zal ik nooit vergeten.

Ik ben je zeer dankbaar dat je me zoveel ruimte hebt gegeven, waardoor ik mijn AIO-periode als zeer prettig heb kunnen beleven.

Albert, in de eerste meetings met jou was ik waarschijnlijk nog wat bedeesd, omdat ik toch een beetje onder de indruk was van je creatieve geest.

Jij bent een man die net als ik van alle vormen van wintersport geniet.

Bedankt voor de kansen die je me tot op de dag van vandaag biedt.

Jan E., ik zie jou als het prototype van een homo universalis.

Bedankt dat ik heb mogen leren van jouw onderwijskundige kennis.

Anne and Uwe, thanks for the nice cooperation

In the future, lets go for further systems integration.

I would also like to thank Julien, Eslam, Rainer, Andries and Hjalmar from Groningen University.

Lets hope that STW grants us some continuity.

Zeker ook bedankt, Hans de draaibank virtuoos.

Hij maakte voor dit project o.a. een zeer geavanceerde doos.

Jan van N., Johan en Daniel de cleanroom technologen, zijn altijd zonder gemopper voor me aan het werk getogen.

Van fabricagemethodes hebben jullie zeker veel begrip, zonder jullie zeker geen werkend Lab-on-Chip. 
Roald, hartelijk dank voor je voorwerk in PicoAspect, jouw ideeën hebben me enorm geholpen in dit project.

Daarnaast ben ik altijd zeer welkom geweest in de groep van professor Han. Erg jammer dat ik jouw niet wat betere Mass-Spec resultaten overhandigen kan.

Vele studenten zijn gekomen en weer vertrokken.

Bij alle was ik hopelijk altijd even betrokken.

Lars, Jochem, Floris, Ewout, Karel, Stefan, Alex en Martin, werken met jullie was een fijne manifestatie.

Hier en daar leidde het zelfs tot een enkele publicatie!

Dirk-Jan, Maarten, Maarten en Arjan knutselden een lab monitorsysteem in elkaar.

Ook het werken met jullie vond ik zeker geen bezwaar.

Met alle studenten heb ik erg fijn samengewerkt, Ik hoop dat ik jullie tegelijkertijd in het leerproces heb versterkt.

Loes, Evelien, over de leuke kanten van ons werk wil ik zeker niet zeuren, maar op Zuid-Korea liep ik na slecht nieuws toch wat te treuren.

Ik zal jullie steun van toen zeker niet vergeten, evenals de actie waarbij we levende vis hebben gegeten.

Justyna, Maarten, two valued electrochemical partners in crime.

I've very much enjoyed our Hawaiian time.

Ulrik Jurva and Serge Lemay, I would like to thank you for your enthousiastic reaction.

I'am looking forward towards the defense reflection.

In dit dankwoord mogen ook zeker niet ontbreken twee malloten, te weten Paul en Wouter mijn kamergenoten.

Paul, als jij in de buurt bent is het nooit stil,

Uitgezonderd een enkel moment, vond ik dat meestal wel chill.

Wouter, bedankt dat ik de kunst der AIO-zijn bij jou heb kunnen afkijken.

Zonder jou had ik zeker niet zoveel kunnen bereiken.

Jullie staan allebei altijd voor me klaar en daarom voor mij onvervangbaar.

Ook bedankt Floor en Iris de werkweek 2008 mede-organisatoren en Erik F., Cora en Ferdi de IEEE senioren.

Ook de samenwerking met alle mensen uit het NLT DOT, was voor mij een waar genot.

Ik wil heel (voormalig) BIOS; Ad, Adithya, Ana, Andries, Arpita, Bianca, Dietrich, Eddy, Edwin, Egbert, Erik K., Fleur, Ganesh, Georgette, Henriette, Istvan, Jacob, 
Lennart, Lingling, Lonneke, Mingliang, Muhammad, Rerngchai, Rogier, Severine, Songyue, Svetlana, Susan, Verena en Yanbo bedanken voor de hulp en gezelligheid, al zijn jullie mij de komende vier jaar nog niet kwijt. Hermine ook jou ben ik dankbaar, omdat je van alles voor me regelde zonder bezwaar.

M'n familie heeft me altijd weten te steunen, ten eerste zijn er mijn ouders op wie ik heb kunnen leunen.

Ook mijn grootouders, broers, (schoon)zussen en schoonfamilie zijn altijd erg betrokken geweest,

maar van jou Marije, houd ik toch het allermeest.

Ik hoop dat ik niemand achterwege heb gelaten, mocht het toch zo zijn dan hoop ik dat je me er niet om zult haten.

Tot slot kan ik zeggen zonder overdrijven, dat ik blij ben dat ik niet mijn gehele proefschrift zo heb hoeven schrijven. 